\title{
A User's Manual for the Oak Ridge Tokamak Transport Code
}

\author{
J. K. Munro \\ J. T. Hogan \\ H. C. Howe \\ D. E. Arnurius
}

\section{OAK RIDGE NATIONAL LABORATORY}




\section{DISCLAIMER}

This report was prepared as an account of work sponsored by an agency of the United States Government. Neither the United States Government nor any agency Thereof, nor any of their employees, makes any warranty, express or implied, or assumes any legal liability or responsibility for the accuracy, completeness, or usefulness of any information, apparatus, product, or process disclosed, or represents that its use would not infringe privately owned rights. Reference herein to any specific commercial product, process, or service by trade name, trademark, manufacturer, or otherwise does not necessarily constitute or imply its endorsement, recommendation, or favoring by the United States Government or any agency thereof. The views and opinions of authors expressed herein do not necessarily state or reflect those of the United States Government or any agency thereof. 


\section{DISCLAIMER}

Portions of this document may be illegible in electronic image products. Images are produced from the best available original document. 


\section{Printed in the United States of America. Available from National Technical Information Service U.S. Department of Commerce 5285 Port Royal Road, Springfield, Virginia 22161 \\ Price: Printed Copy $\$ 10.00$; Microfiche $\$ 3.00$}

This report was prepared as an account of work sponsored by the United States Government. Neither the United States nor the Energy Research and Development Administration/United States Nuclear Regulatory Commission, nor any of their employees, nor any of their contractors, subcontractors, or their employees, makes any warranty, express or implied, or assumes any legal liability or responsibility for the accuracy, completeness or usefulness of any information, apparatus, product or process disclosed, or represents that its use would not infringe privately owned rights. 
Contract No. W-7405-eng-26

FUSION ENERGY DIVISION

\section{A USER'S MANUAL FOR THE OAK RIDGE} TOKAMAK TRANSPORT CODE

J. K. Munro

J. T. Hogan

H. C. Howe

D. E. Arnurius

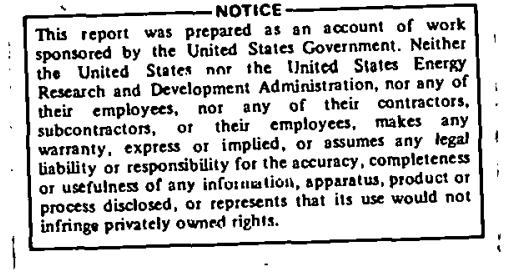

NOTICE

This document contains information of a preliminary nature. It is subject to revision or correction and therefore does not represent a final report.

Date Published: February 1977

Prepared by the OAK RIDGE NATIONAL LABORATORY

Oak Ridge, Tennessee 37830

operated by

UNION CARBIDE CORPORATION

for the

ENERGY RESEARCH AND DEVELOPMENT ADMINISTRATION

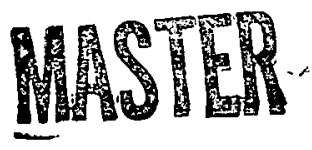




\section{THIS PAGE}

\section{WAS INTENTIONALLY \\ LEFT BLANK}


CONTENTS

FOREWORD ............................. . ix

ABSTRACT . . . . . . . . . . . . . . . . . . . xi

1. INTRODUCTION . . . . . . . . . . . . . . . . 1

1.1 General Structure of Program Spatial-Time Grid . . . . . 2

1.2 Use of Switches and Default Values . . . . . . . . . 2

1.3 Brief Description of Modular Structure . . . . . . . 5

1.4 Description of Input Data and Default Values . . . . . . 6

1.5 Comments on Use of NAMELIST Feature for Input Data . • . 14

1.6 Programming Considerations . . . . . . . . . . . 14

REFERENCES . . . . . . . . . . . . . . . . . . 15

2. DESCRIPTION OF MAIN PROGRAM MODULE AND SUPPORTING

SUBROUTINES . . . . . . . . . . . . . . . . . 17

2.1 Overview of Code Structure . . . . . . . . . . 17

2.2 Initialization Procedure . . . . . . . . . . . 17

2.3 Balances and Histories . . . . . . . . . . . . 19

2.4 Graphics Options . . . . . . . . . . . . . . . 20

2.5 Descriptions of Subroutines . . . . . . . . . . 20

Routine MAIN ................ 20

Subroutine GETSET ................ 20

Subroutine $\emptyset$ RMPWR ............... 26

Entry SAVPWR ............... 26

Entry NEWPWR .............. 26

Function PC ............... 33

Subroutine PRT $\emptyset \mathrm{UT}$. . . . . . . . . . . 34

Gubroutinc RESET . . . . . . . ....... . 35

Subroutine SIGMA ............... 37

Subroutine TIMSTP .............. 39

REFERENCES . . . . . . . . . . . . . . . . . . 41

3. DESCRIPTION OF PLASMA MODULE . . . . . . . . . . . . . 43

3.1 Purpose and Structure of Modulc . . . . . . . . . . . 43

3.2 Basic Input/Output . . . . . . . . . . . . 43

3.3 Summary of Function of Each Subroutine . . . . . . . . 44

Subroutine PLASIM ............. 44

Entry IPPC ............... 44

Entry PLASMA ............... . 44

Subroutine CDC ................. 46

Subroutine CST . . . . ............. 55

Subroutine LBM ................ 56

Subroutine IBTM .............. 63 
REFERENCES . . . . . . . . . . . . . . 64

4. DESCRIPTION OF NEUTRAL GAS MODULE . . . . . . . . . 65

4.1 Purpose and Structure of Module . . . . . . . . 65

4.2 Basic Input/Output . . . . . . . . . . . 68

4.3 Description of the Analytic Model for the Total

Neutral Density . . . . . . . . . . 6 68

4.4 Slab Model Approximation to the Neutral Gas Density

Without Wall Reflection............ 69

4.4.1 Neutral gas density as the solution to an

integral equation ........... . . 69

4.4.2 Computational procedure for the determination

of an approximation to the hot neutral density,

$\mathrm{N}_{1} \ldots \ldots \ldots \ldots 71$

4.4.3 Computational description of the approximation

to $\hat{I}\left(g, x_{j}\right) \ldots \ldots 73$

4.4.4 Required input, space allocation, and calling

sequence for computer program ........ 75

4.5 Summary of Function of Each Subroutine . . . . . . . 77

Subroutine NEUTRA .......... 78

Subroutine NTF . . . . . . . . . . . . 79

Subroutine $\mathrm{T} \emptyset \mathrm{C} \emptyset \mathrm{MP}$. . . . . . . . . 80

Subroutine SPECl' ............ 81

Subroutine NEUTIT .......... 82

Subroutine NEUTIO . . . . . . . . . . 83

Subroutine NUKERN ........... . . 84

Subroutine NEUTC ............. 85

Function T̄DN ............ 86

Function NO . . . . . . . . . . 87

Subroutine DEIM $\phi D$. . . . . . . . 88

Function N1INTP ............ . . 89

Subroutine BINSEE ........... 90

Function E2 .................... 91

Function $\mathrm{E} 1$............... 92

Function G............. 93

Function $G M$.............. . . 94

Function NI . . . . . . . . . . . . . . 95

Function T.............. 96

Function NIDERT . . . . . . . . . . 97

Function SRTT ............. . . . 98

Function NIINT ............. . . 99

Subroutine NIIEQL . . . . . . . . . . . 100

Subroutine NIIEQR . . . . . . . . . 101

Function EATN ............ 102

Subroutine SSIMP ........... 103

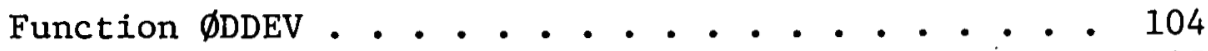

Function INTRPU ............. . . 105

Subroutine ERR $\emptyset$........... 106

REFERENCES . . . . . . . . . . . . . . 107 
5. DESCRIPTION OF THE IMPURITY MODULE . . . . . . . . . . 109

5.1 Purpose and Structure of Module . . . . . . . . . . 109

5.2 Basic Input/Output ................. 110

5.3 Summary of Models Available . . . . . . . . . . 110

5.4 Brief Description of Each Subroutine in the Impurity

Module ..................... 110

Subroutine IMPRTY .............. 111

Function FCT ................. 115

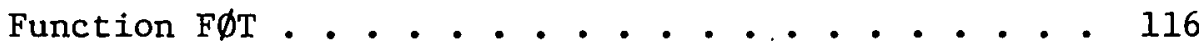

Function FCARX .................. 117

Function $F \emptyset X$.................. 118

Subroutine TABLE . . . . . . . . . . . 119

Subroutine ZCALC . . . . . . . . . . . 120

Subroutine ZDIFFU . . . . . . . . . . . . . 124

Subroutine EVALC . . . . . . . . . . . . 127

Subroutine $\mathrm{CDZ}$. . . . . . . . . . . . 128

Subroutine EVALK . . . . . . . . . . . 130

Subroutine CØEFF ............... 131

Subroutine SØLVE . . . . . . . . . . . . 133

Function HZR ................ . 134

Subroutine HIGHZ . . . . . . . . . . 135

Subroutine DYNAM . .............. 136

Subroutine SV $\varnothing X Y G$............. 138

Subroutine SVCARB ............. 139

REFERENCES . . . . . . . . . . . . . . . . . . . . . 140

6. DESCRIPTION OF NEUTRAL BEAM INJECTION MODULE . • . • • • • • . 141

6.1 Purpose, Structure, and Basic Input/Output of Module • • 141

6.2 Summary of Function of Each Subroutine . . . . . . . . . 142

BLØCK DATA ................ 142

Subroutine BEAM ................ 145

Subroutine BMPWR ................ 151

Subroutine QUADR ................. 154

Subroutine SUBH ............... 155

Subroutine AVETH ............... 159

Subroutine RPT3XY ............... 162

Subroutine W3XYZ .............. 165

Subroutine GAUSST . . . . . . . . . . . 168

Subroutine GAUSST . . . . . . . . . . . 170

Function XTERP . . . . . . . . . . . . 171

Function YTERP ................. 172

Subroutine FPM $\emptyset \mathrm{ME}$. . . . . . . . . . . 173

Function VEL . . . . . . . . . . . . 177

Function TCX ................. 178

Function PCX .................. 179

Function PCFUN . . . . . . . . . . . . . 180

Funclion GIF . . . . . . . . . . . . . . . 181

Function GIFUN . . . . . . . . . . . . . . 182

Function $\mathrm{CEF}$. . . . . . . . . . . . 183 


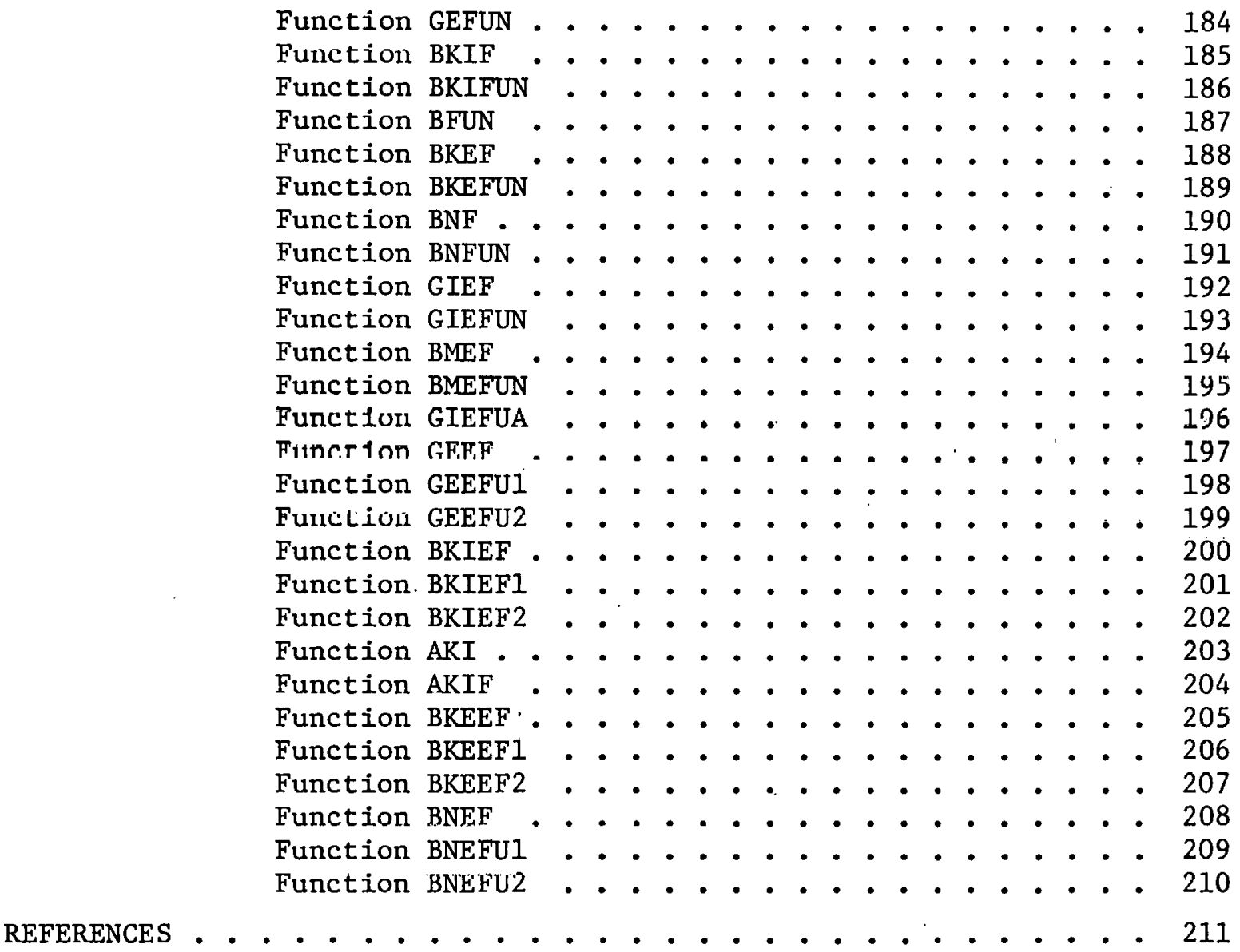

7. DESCRIPTION OF SURFACE MODULE . . . . . . . . . . . . . 213

7.1 Purpose and Structure of Module . . . . . . . . . . 213

7.2 Basic Input/Output . . . . . . . . . . . . . 216

7.3 Summary of function of Each Subroutine . . . . . . . . 217

Subroutine SURFAC ............. 217

8. DESCIRIPTION OF DIACNOSTIC MODULE . . . . . . . . . . . . 219

8.1 Purpose and Structure of Module . . . . . . . . . . 219

8.2 Summary of Function of Each Subroutine . . . . . . . . 220

Subroutine DIAGN $\emptyset$............. 220

Entry DAPRT ................ 220

Entry DPKT .............. 220

APPENDIX I. DEFAULT VALUES . . . . . . . . . . . . . . . I.1

APPENDIX II. COMMON BLOCKS . . . . . . . . . . . . . . . II.1

A. Names and Primary Functions of Labeled
Common Blocks . . . . . . . . . . . II.2

B. Identification of Variables and Arrays in 
APPENDIX III. EVALUATION OF SELECTED MATRIX ELEMENTS . • . . . . . III.1 APPENDIX IV. REFERENCE VERSION OF ØRTTC: THE OAK RIDGE TOKAMAK TRANSPORT CODE . . . . . . . . . . . . . . . IV.I 


\section{THIS PAGE}

\section{WAS INTENTIONALLY \\ LEFT BLANK}


FOREWORD

This document is intended as a starting point for a continuing documentation of the Oak Ridge Tokamak Transport Code. The code has been developed by scientists whose chief interest lies in the analysis of tokamak experiments and the formulation of reliable models of extrapolation. Consequently, potential users are cautioned that the version of the code described here is far from optimum in the computational sense. Some present versions of the code, incorporating most of the physical models described in this manual, have halved both execution time and storage requirements. Nonetheless, this manual contains such a detailed description that users who require greater efficiency have the information needed to obtain it.

As this manual is written, the Oak Ridge Tokamak Transport Code includes some features not described here. These include:

a) a Fokker-Planck solution to the fast injected ion distribution function at $N_{i} \sim 5-10$ radial points (that is, $N_{i}$ solutions of the two-dimensional time-dependent equations for $f_{i}\left(x_{i}, v, \xi, t\right)$.

b) a multi-group, discrete ordinate $\left(S_{N}\right)$ cylindrical geometry calculation of neutral gas transport. This NUTRLSN package has been developed by modifying the XSDRN neutron and photon transport solver which has been extensively used in other fields.

c) a quasi-two-dimcnsional magnetic geometry. In this scheme the $(r, t)$ radial diffusion problem is replaced by one solved on $a(\psi, t)$ grid where $\psi$ is the poloidal flux function. The ISLAND code of D. C. Stevens (as used in the work of Grad, Hu, and Stevens) is then employed to compute the two-dimensional axisymmetric magnetic surface structure.

These additional modules are run with the same basic structure described in this manual and the logic has been designed to accept them. It is expected that documentation of these more sophisticated features will soon be forthcoming. 


\section{THIS PAGE}

WAS INTENTIONALLY

LEFT BLANK 
A one-dimensional tokamak transport code is described which simulates a plasma discharge using a fluid model which includes power balances for electrons and ions, conservation of mass, and Maxwell's equations. The modular structure of the code allows a user to add models of various physical processes which can modify the discharge behavior. Such physical processes treated in the version of the code described here include effects of plasma transport, neutral gas transport, impurity diffusion, and neutral beam injection. Each process can be modeled by a parameterized analytic formula or at least one detailed numerical calculation. The program logic of each module is presented, followed by detailed descriptions of each subroutine used by the module. The physics underlying the models is only briefly summarized. The transport code was written in IBM FøRTRAN-IV and implemented on IBM $360 / 370$ series computers at the Oak Ridge National Laboratory and on the CDC 7600 computers of. the Magnetic Fusion Energy (MFE) Computing Center of the Lawrence Livermore Laboratory. A listing of the current reference version is provided on accompanying microfiche. 


\section{A USER'S MANUAL FOR THE OAK RIDGE TOKAMAK TRANSPORT CODE*}

\section{INTRODUCTION}

\subsection{GENERAL STRUCTURE OF PROGRAM SPATIAL-TIME GRID}

This manual is intended for a user who wishes to change the basic physical model in the code for one or more processes, or to incorporate a model for some process not already treated in order to study its interaction with the basic plasma transport process.

The manual can be most effectively used with two other documents: ${ }^{1,2}$

J. T. Hogan, "Multi-Flu1d Tokamak Transport Models," ORNL/TM-5153 (November 1975); Methods of Computational Physics, Vol. 16: Computer Applications to Controlled Thermonuclear Research, ed. by J. Killeen, B. Alder, and S. Fernbach (1976).

J. T. Hogan, Physical Models in the Oak Ridge Tokamak Transport Code, ORNL/TM, to be published.

The reference version of the Oak Ridge Tokamak Transport Code ( $\varnothing$ RTTC) is supplied in Appendix IV on microfiche.

The code is available to members of the controlled fusion community via the Magnetic Fusion Energy (MFE) Computer Center at the Lawrence Livermore Laboratory.

The tokamak transport code was developed as a tool to aid both theorists and experimentalists working with tokamak-type toroidal plasma machines. This code has been structured to allow modular incorporation of new models of various plasma processes as they are developed. The code has been extensively documented internally to aid users who must know the details of the processes being modeled. For those who wish to use the code to lielp inlerpret data, the input data is arranged so as to allow for the inclusion of extensive comments in the actual input data without disturbing the normal running of the program.

\footnotetext{
* Research sponsored by the Energy Research and Development Administration
} under contract with Union Carbide Corporation. 
The input data is grouped as much as possible to reflect the modular structure of the program. This parallel organization should facilitate the selection of options for a particular type of simulation calculation. Where possible, an attempt has been made to include at least two alternatives for the important processes modeled: an analytic model that provides a rough approximation but gives a quick calculation, and a more detailed numerical computation which treats the physics of the process with more care.

The program can be run with a set of default input values. since there are many combinations of options available, a default combination has been shnsen which will be designated the standard choice. The "standard choice" data set is also a useful reference point if the user attempts to use a combination of input values for which the code produces numerically unstable profiles. A poor choice of input data values may result in difficulty in getting a simulation started; i.e., problems may develop in the first few time steps and cause the profiles to behave strangely or to grow rapidly to large magnitudes. The basic spatial and time intervals for the standard choice data set are $0.02 \times$ (plasma radius, $\mathrm{cm}$ ) and tens of microseconds, respectively. Some useful guidelines to consider in putting together a data set that ehould not lead to numerical difficulty are summarized in Sect. 1.4.8. Models currentily under development are not treated in this manual, but a logical structure which will accommodate them has been chosen for the code. Some examples of models not covered here are:

- computation of sulutions to a two-dimensionall Fokker-Planck equation for the neutral beall injection module

- use of the NUTRLSN neutron transport code for the neutral gas module

- Lwuedimensional flux surface calculations ${ }^{3}$

- the collisional plasma model ${ }^{4}$ to replace the plasma diffusion equations.

\subsection{USE OF SWITCHES AND DEFAULT VALUES}

The code uses a number of switches for a variety of purposes. These switches can be grouped according to their purpose and/or use into the following types: 
Type

Purpose and/or Use

A Determines choice of particular model of a process

B Controls input and/or. output

C Determines choice of machine configuration, e.g., neutral beam injection

D Provides choice of diagnostic options

E Provides values of exponents for power law expressions.

A detailed description of the various switches is given in Table 1 .

Table 1. Summary of switch types and the

function or use of each switch

Type A Switches: Choice of analytic or detailed numerical model

NUTE(1) Neutral gas calculation

$=1$, algebraic formula

$=2$, Boltzmann transport model with slab geometry, no wall reflection

$=3$, same as NUTE $(1)=2$, but with wall reflection

$=4$, NUTRLSN transport code

Options 3 and 4 are not included in the Reference Version (see Appendix IV)

IMP(1) Initial impurity stripping calculation

$=1$, corona equilibrium table for carbon and oxygen

$=2$, rate equation solutions for carbon and oxygen

IMP (2) Radial distribution of total impurities

$=1$, corona equilibrium tables together with radial distribution formulae for carbon and oxygen. High-Z impurities are treated using a crude estimate.

$=2$, corona equilibrium tables for carbon, oxygen, and high-Z impurity ( $\mathrm{Fe}$ ) atom charge states. Radial distributions are assigned using formulae.

$=3$, corona equilibrium tables together with radial distributions generated by Pfirsch-Schliuter diffusion for carbon and oxygen. High-Z impurities are treated using a crude estimate.

= 4, corona equilibrium tables for carbon, oxygen, and high-Z impurity ( $\mathrm{Fe}$ ) atom charge states. Radial distributions are generated by a Pfirsch-Schlüter diffusion model. 
Table 1 (continued).

IMP(3) Impurities: Fixed values $(=1)$ or detailed calculation $(=2)$ according to the value of IMP(2).

IMP (4) Detailed model for iron

$=0$, include iron coronal equilibria

$=1$, omit detailed description

INJ(3) Current perturbation from injected neutral beam

INJ(4) Electric field effect on fast ions

INJ(5) Luss cune currecliun to fast fon thermalization

INJ(6) Neutral injection gas

$=0$, hydrogen

$=1$, deuterium

Type B Switches: I/ $\varnothing$ control

INIT(1) Generate initial plasma profiles analytically or read them in/restart calculation at some arbitrary time.

$=0$, generate initial profiles analytically ("normal" start)

$=1$, read in initial profiles

$=2$, restart calculation at a designated time

Type f: Switches: Machine configuration

INJ(1) Neutral beam injection

$=0$, no injection

$=1$, moments calculation

$=2$, debug calculation

INJ(2) Number and type of neutral beam injection used

$=1$, co-injection only

$=2$, counter-injection only

$=3$, two injectors, one co- and one counter-

$=4$, four injectors, two co- and two counter-

Type D Switches: Diagnostic options

INIT(4) Diagnostic options switch

$=0$, off

$=1$, on

Type E Switches: Exponents and power laws used

NUTE(2) Exponent in power law for neutral gas analytic model. 
A standard set of default input values has been incorporated into the code; the entire set is given in Appendix $I$. The choice of default value for each input variable is explained in the detailed description of the input data (Sect. 1.4). The default values for the type A switches will generally pick the analytic model. Type B switches will be set to generate the initial plasma profiles analytically and to provide detailed printout.

\subsection{BRIEF DESCRIPTION OF MODULAR STRUCTURE}

Each of the seven basic modules currently in the code is discussed in a separate section. Each section includes a description of the input data required and a summary of the function of each subroutine used by the module. These modules and their functions are briefly as follows:

\footnotetext{
MAIN - reads the Input data and provides primary program control (Sect. 2.5)

PLASMA - obtains the solution for the set of partial differential equations describing the time evolution of the plasma (Sect. 3)

NEUTRA - calculates the effects of the inward-diffusing cold neutral gas (Sect. 4)

IMPRTY - calculates the effects due to the presence of the impurities carbon and oxygen and a high-z element (Fe) (Sect. 5)

BEAM - models the neutral beam injection process (Sect. 6)

DIAGN $\emptyset$ - generates diagnostic tests to measure the accuracy and performance of the simulation (Sect. 7)

SURFAC - models the interaction between the plasma surface and the external world (diverters, torus walls, etc.)(Sect. 8).

Labeled common blocks have been used extensively to store variables and arrays whose values are used or generated in more than one module or subroutine. The common blocks were constructed to parallel the modular structure of the transport code as much as possible. Common block names were chosen to suggest a common physical use or association of the variables and arrays grouped in each block. A list of the variables (arrays) in each common block is given in Appendix II, together with variable and
} array definitions. 


\subsection{DESCRIPTION OF INPUT DATA AND DEFAULT VALUES}

An input data set, completely internally documented, is presented in Appendix I to enable the user to run the plasma simulation code without having to consult a series of memos. Changes to the input data can be made by editing the appropriate NAMELIST 1ist in this input data set. The standard choice, with complete documentation, is built into BL $\emptyset \mathrm{CK}$ DATA, so that it will always be available in the code for reference. AlI input data appears in the labeled common blocks.

In the sample input data set, variables appropriate for each NAMELISI' list grouping are described immediately preceding the NAMELIS'L' data set. Units of the input variables are given with their definitiuns. Default values for the input data are given in parentheses following the definitions of the variables; these values are the ones appropriate for the ORMAK. machine.

1. Machine Configuration. The parameters associated with the tokamak geometry (ORMAK values are the default) are:

$$
\begin{array}{ll}
\mathrm{AM} \text { - minor radius of torus, cm } & (23.0 \mathrm{~cm}) \\
\mathrm{R} \emptyset \text { - major radius of torus, cm } & (79.8 \mathrm{~cm}) \\
\mathrm{BT} \text { - toroidal magnetic field, G } & (18 \mathrm{kG}) \\
\mathrm{R} 9^{*} \text { - initial plasma radius, cm } & (23.0 \mathrm{~cm}) \\
\mathrm{N} \text { - number of spatial grid (radial) points } & (51)
\end{array}
$$

\begin{tabular}{|c|c|c|}
\hline TMAX & $\begin{array}{l}\text { - maximum duration of current pulse, } \\
\text { msec }\end{array}$ & $(65.0 \mathrm{mscc})$ \\
\hline TC + & - rise time of current, msec & $(10.0 \mathrm{msec})$ \\
\hline $\operatorname{li} Y \phi^{+}$ & $\begin{array}{l}\text { - fall off time of external neutral } \\
\text { density, msec }\end{array}$ & $(4.0 \mathrm{msec})$ \\
\hline TSTAR & $\begin{array}{l}\text { - starting time of simulation prob- } \\
\text { lem, msec }\end{array}$ & $(0.0 \mathrm{msec})$ \\
\hline NT & $\begin{array}{l}\text { - total number of time intervals for } \\
\text { current pulse and plasma simulation }\end{array}$ & $(1000)$ \\
\hline
\end{tabular}

2. Timing Intervals. Two groups of timing intervals appear in the code. The first group listed below deals with the basic plasma model. The second group is associated with the neutral beam injection module and appears with the input for that module in Sect. 7.

\footnotetext{
* See subroutine GETSET.

${ }^{\dagger}$ See subroutine SURFAC.
} 
3. Plasma Data. This group of input variables is used primarily by the plasma module for generating the initial plasma variable profiles.

\begin{tabular}{|c|c|c|}
\hline $\operatorname{TE} \emptyset$ & $\begin{array}{l}\text { - boundary temperature for electrons, } \\
\mathrm{eV}\end{array}$ & $(10.0 \mathrm{eV})$ \\
\hline $\mathrm{TI} \emptyset$ & - boundary temperature of ions, $\mathrm{eV}$ & $(10.0 \mathrm{eV})$ \\
\hline TEB & $\begin{array}{l}\text { - initial central value of electron } \\
\text { temperature, eV }\end{array}$ & $(20.0 \mathrm{eV})$ \\
\hline TIB & $\begin{array}{l}\text { - initial central value of ion } \\
\text { temperature, eV }\end{array}$ & $(20.0 \mathrm{eV})$ \\
\hline $\mathrm{V} \emptyset \mathrm{LT}$ & - toroidal voltage, $\mathrm{V}$ & $(3.0 \mathrm{~V})$ \\
\hline DEN $\emptyset$ & $\begin{array}{l}\text { - initial central value of plasma } \\
\text { electron density, } \mathrm{cm}^{-3}\end{array}$ & $\left(6 \times 10^{12} \mathrm{~cm}^{-3}\right)$ \\
\hline DENB & - boundary electron density, $\mathrm{cm}^{-3}$ & $\left(5 \times 10^{12} \mathrm{~cm}^{-3}\right)$ \\
\hline ZPC & $\begin{array}{l}\text { - initial value of total current, } \\
\text { amps }\end{array}$ & \\
\hline ZFI & $\begin{array}{l}\text { - additional amount of total current } \\
\text { after time TC, amps* }\end{array}$ & $(85.0 \mathrm{kA})$ \\
\hline AMU & $\begin{array}{l}\text { - mass of plasma ions, amu, deter- } \\
\text { mined according to the equation: }\end{array}$ & \\
\hline & $\begin{array}{l}\mathrm{AMU}=\left(2^{*} \mathrm{~N}_{\mathrm{D}+}+\mathrm{N}_{\mathrm{H}+}\right) /\left(\mathrm{N}_{\mathrm{D}+}+\mathrm{N}_{\mathrm{H}+}\right) \\
\text { where } \mathrm{N}_{\mathrm{D}+} \text { and } \mathrm{N}_{\mathrm{H}+} \text { are the respective } \\
\text { densities of deuterons and protons }\end{array}$ & $(1.0 \mathrm{amu})$ \\
\hline $\mathrm{XI}(1)$ & $\begin{array}{l}\text { - exponent for initial profile of cur- } \\
\text { rent density, } \\
\qquad j=j_{0}\left[1-(R / R 9)^{X I(1)}\right]\end{array}$ & $(2.0)$ \\
\hline$X I(2)$ & $\begin{array}{l}\text { - exponent for initial profile of } \\
\quad \text { electron temperature, } \\
T_{e}=(\operatorname{TE} \emptyset-T E B)\left[1-(R / R 9)^{X I(2)}\right]+T E B\end{array}$ & $(2.0)$ \\
\hline $\mathrm{XI}(3)$ & $\begin{array}{l}\text { - exponent for initial profile of ion } \\
\text { temperature, }\end{array}$ & $(2.0)$ \\
\hline & $T_{i}=(T E \emptyset-T I B)\left[1-(R / R 9)^{X I(3)}\right]+T I B$ & \\
\hline XI (4) & $\begin{array}{l}\text { - exponent for initial profile of } \\
\quad \text { electron density, } \\
\qquad \mathrm{N}=(\mathrm{DEN} \emptyset-\mathrm{DENB})\left[1-(\mathrm{R} / \mathrm{R} 9)^{\mathrm{XI}(4)}\right]=\text { DENB. }\end{array}$ & $(2.0)$ \\
\hline $\operatorname{INIT}(1)$ & $\begin{array}{c}\text { - switch for starting mode for } \\
\text { initial plasma variable profiles } \\
=0, \text { for profiles generated } \\
\text { analytically } \\
=1 \text {, for profiles read from } \\
\text { data cards }\end{array}$ & $(0)$ \\
\hline
\end{tabular}

\footnotetext{
See function PC.
} 


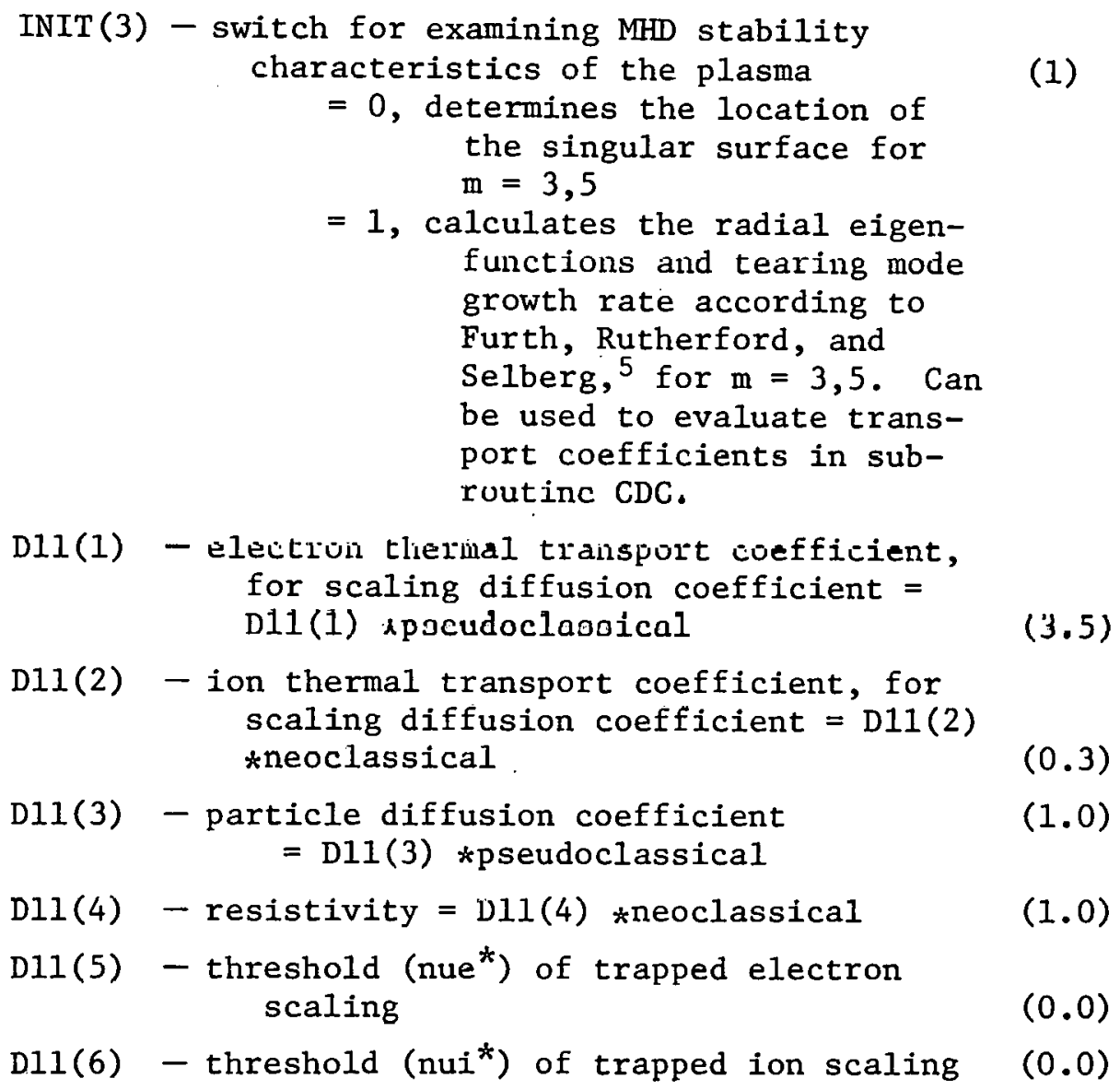

4. Neutral Gas Data. This group of input variables is used almost entirely by the neutral gas module for plcking a path through a consistent choice of options associated with a particular neutral gas model.

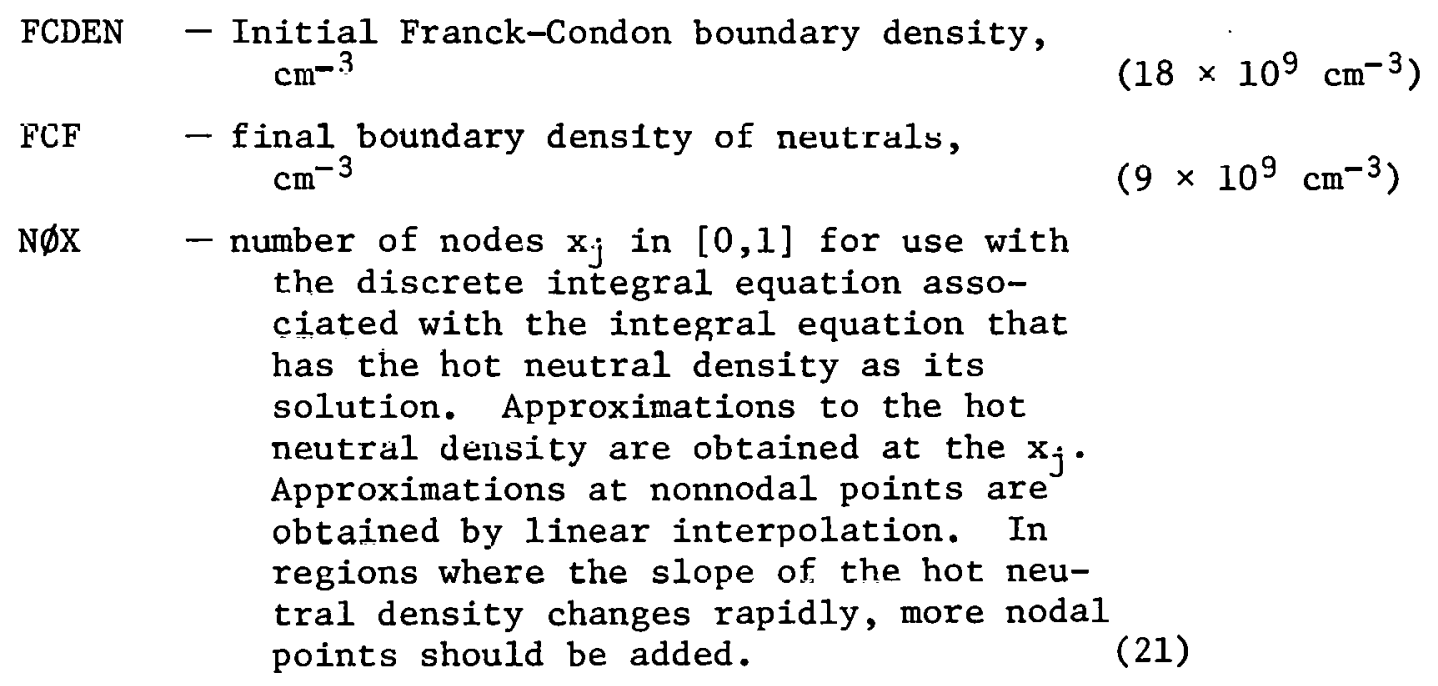




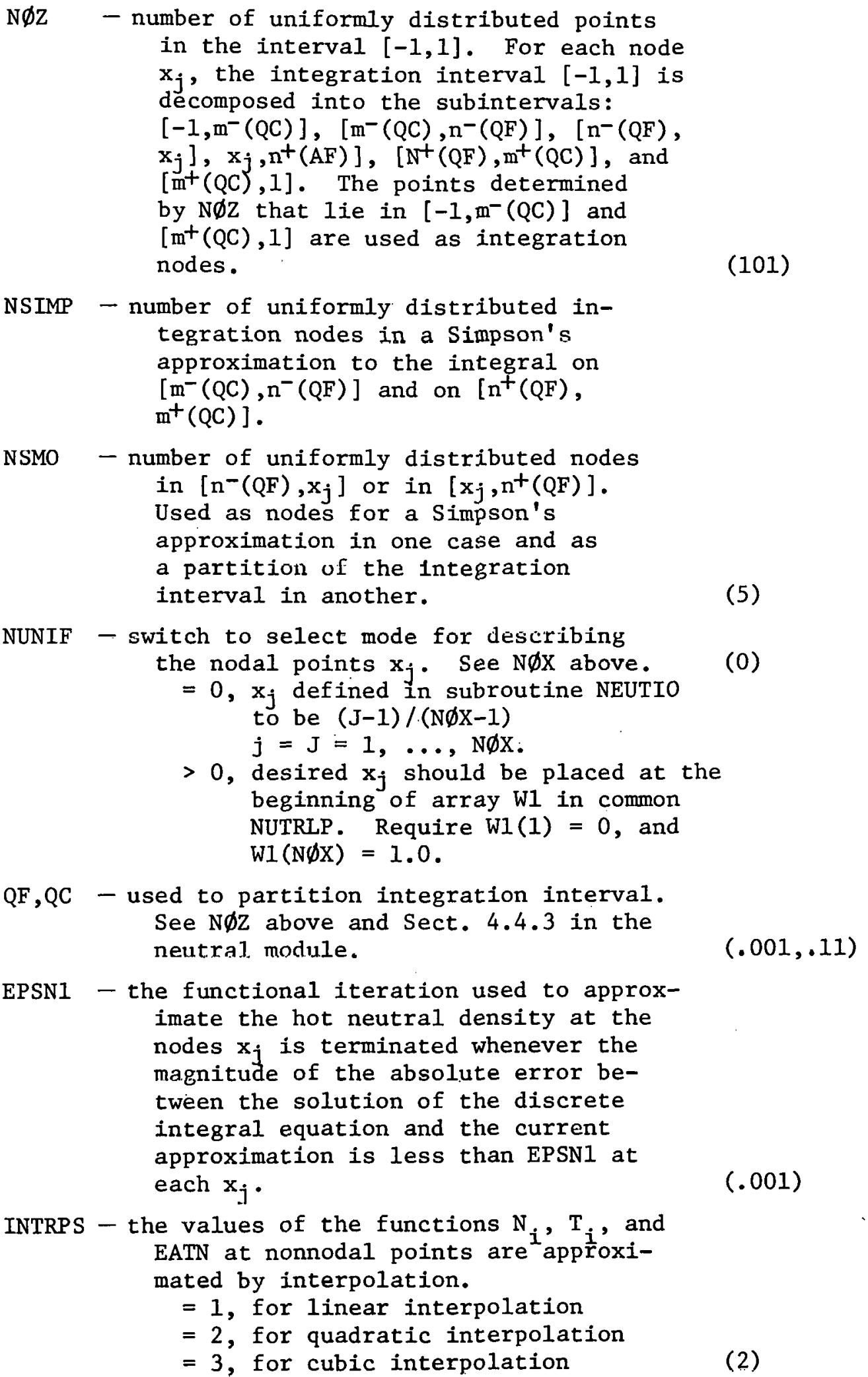




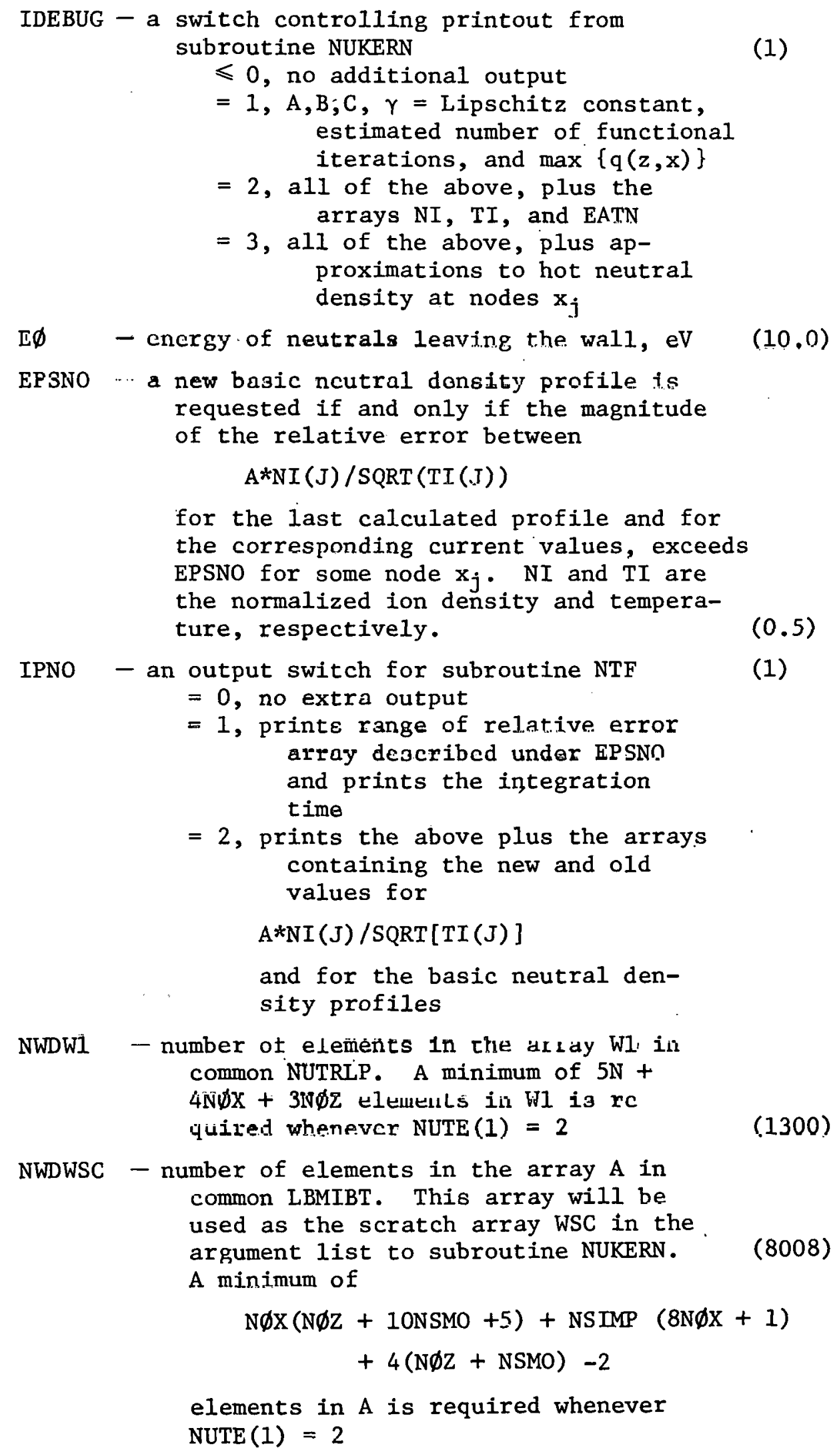




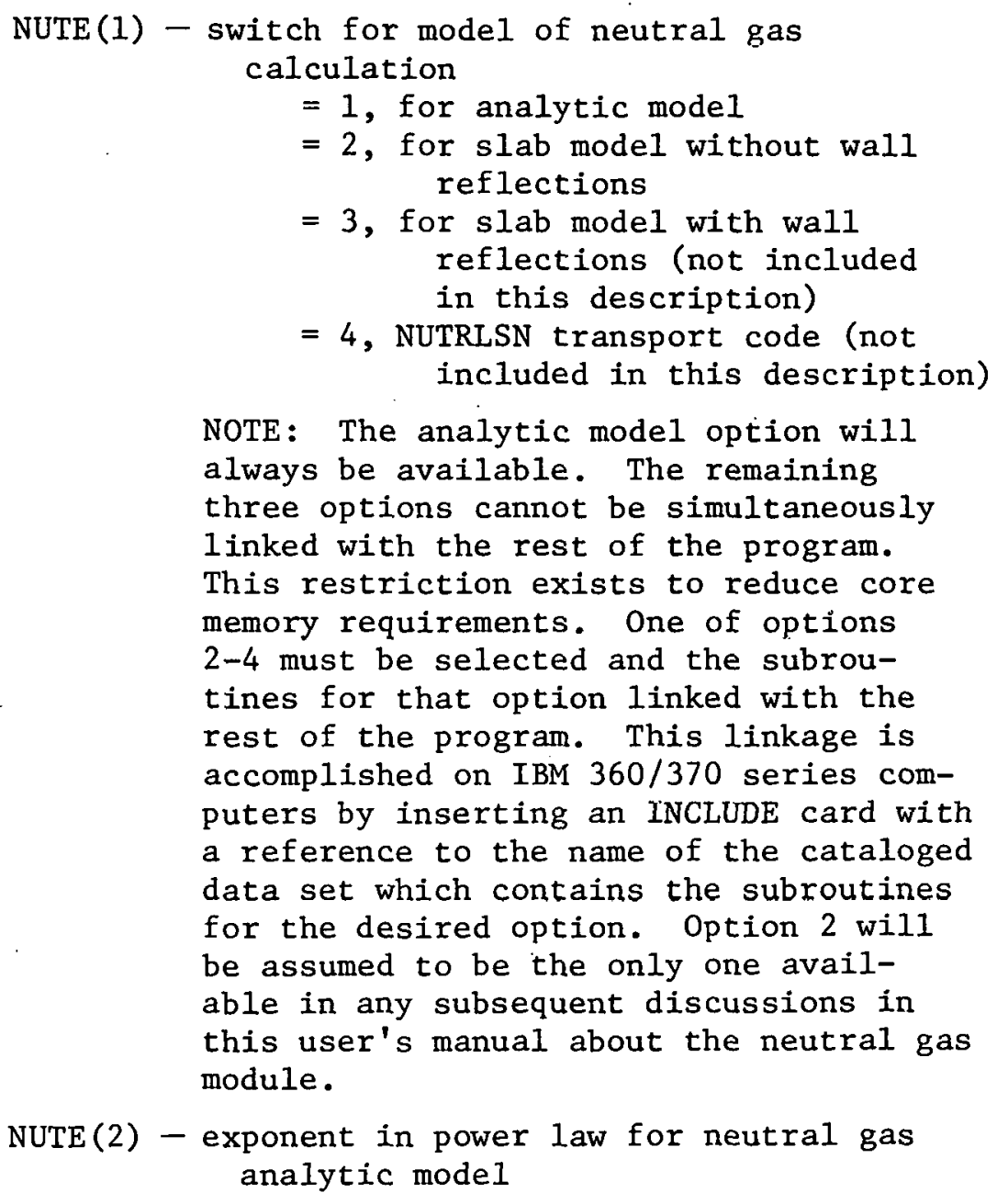

5. Impurity Ion Data. This input data is used to some degree throughout the code, but is most closely associated with the impurity module (Scet. 5).

\begin{tabular}{|c|c|}
\hline CIMP & $\begin{array}{l}\text { - percentage of electron density due to } \\
\text { high-z impurity. This parameter has two } \\
\text { different meanings, according to the im- } \\
\text { purity diffusion model option determined } \\
\text { by the value of IMP (2). For IMP (2) = } 1 \\
\text { or } 3 \text {, PCIMP is due to some average ef- } \\
\text { fective high-Z impurity; for IMP(2) = } 2 \\
\text { or } 4 \text {, it is due to iron impurities }\end{array}$ \\
\hline ZEFF & - effective charge of ions \\
\hline Z IMP & - maximum charge of the high-Z impurity io \\
\hline XCAR & $\begin{array}{l}\text { - percentage of electron density due to } \\
\text { carbon impurities }\end{array}$ \\
\hline$x \ddot{\phi} x$ & - percentage of electron density due to \\
\hline
\end{tabular}




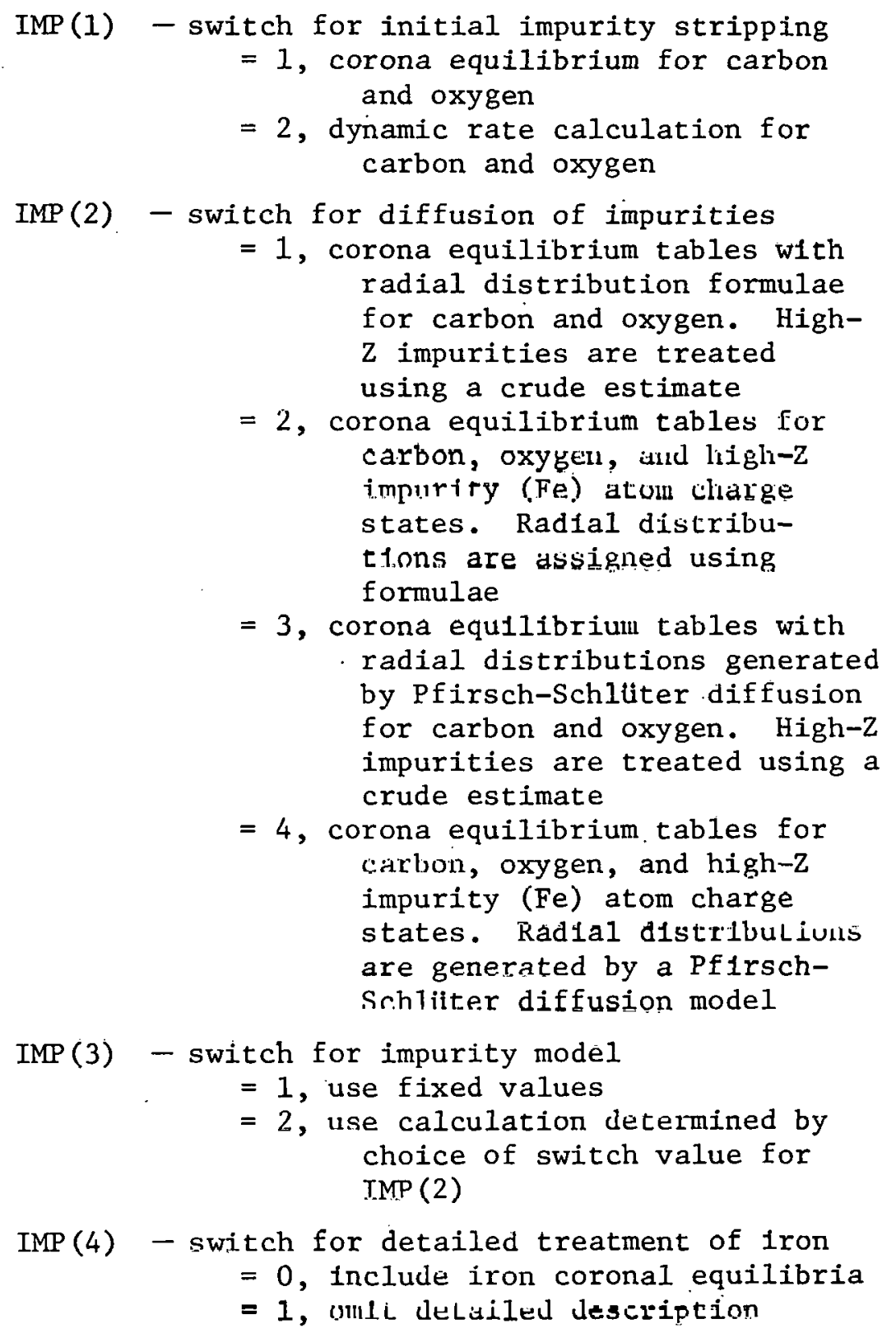

6. Neutral Beam Injection Data. This group of input variables is used almost entirely by the neutral beam injection module for choosing a path through a consistent set of options associated with a particular neutral beam injection model.

$$
\begin{aligned}
& \text { DTCH - time increment for calculation of } \\
& \text { profile } \mathrm{H}(\mathrm{r}) \text {, msec (10.0 msec) } \\
& \text { DTCGL - time increment for calculation of GE, } \\
& \mathrm{GI} \text {, and } \mathrm{KE} \text {, } \mathrm{msec} \text { (2.0 } \mathrm{msec} \text { ) }
\end{aligned}
$$




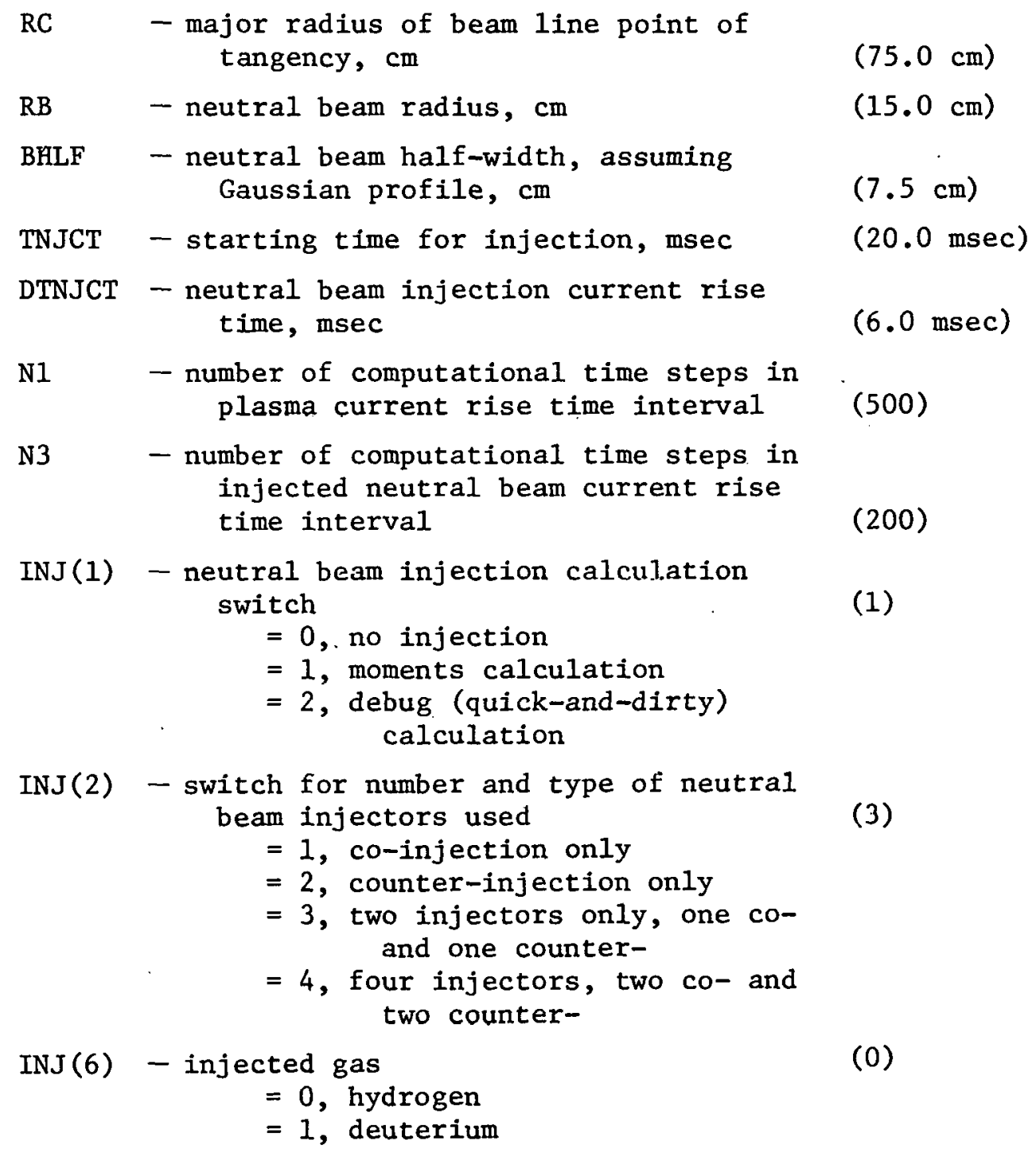

For the following switches, $0=$ off and $1=$ on:

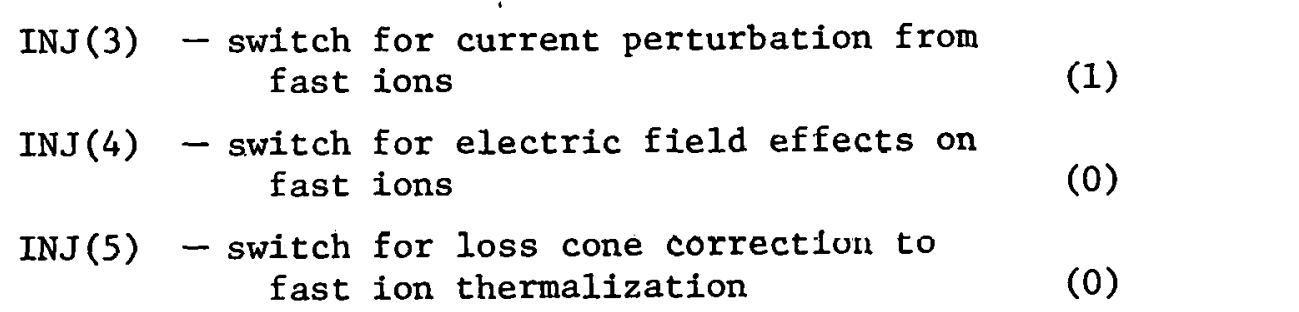

7. I/ Data and Control Switches. This group of variables and switches governs hiow often printout is obtained during the simulation run and * what level of diagnostic information is derived from the diagnostics module.

NR - number of printout sets obtalned during execution of the code 
8. Using Other Data Sets. Data sets with the same numerical structure (i.e., same N, NT, N1, etc.) will have a better chance of success if the ohmic heating input rate chosen initially is close to the one for the reference value. Thus, $n j^{2}$ should be close to the value given here; variations should incorporate the fact that $\eta \sim T_{e}^{-3 / 2}$ and $j \sim$ total current/area.

1.5 COMMENTS ON USE OF NAMELIST FEATURE FOR INPUT DATA

The format for the data in the NAMELIST data sets is as foilnws:

1. Column 1 must always remain blank.

2. A set of data associated with a particular NAMELIST list name must begin with an ampersand in column 2 followed immediately by the NAMELIST name. The set of data must end with a line containing an ampersand in column 2 followed immediately by END.

3. Values for single variables are entered, beginning in column 2, according to the format:

$$
\text { VAR1 = valuel, VAR2 = value2, etc. }
$$

4. Values for arrays may be entered in two ways:

a. individually:

ARRAY (1) = valuel, $\operatorname{ARRAY}(3)=$ value 3 , etc.

b. in a list:

ARRAY = value1, value2, value 3 , etc.

After the input data has been read in, it can be written back out using the NAMELIS' feature. If a new variahle (array) is anded to the input data, the appropriate NAMELIST statement defining the variables (arrays) associated with the NAMELIST name must have the variable (array) name added to it. These statements all occur in the group of nonexcutable statements at the beginning of subroutine GFTSFT.

\subsection{PROGRAMMING CONSIDERATIONS}

The language ueed io FøRTRAN IV. The code was developed using the IBM 360 series computers and the $\emptyset \mathrm{PT}=2$ version of the FøRTRAN-H compiler. While the ORNL IBM $\emptyset \mathrm{S} / 360$ F $\emptyset \mathrm{RTRAN}-\mathrm{H}$ compiler can accept statements such as:

$$
\mathrm{XK}=(\mathrm{I}-1) * 1 . / \mathrm{NML} / \mathrm{NM1},
$$


and can handle the mixed mode arithmetic properly, other compilers do not have this capability. An attempt has been made to weed out this type of machine-dependent statements and to replace these statements with sequences of statements that should not cause difficulty on most compilers. We have tried to use ANSI standard FøRTRAN whenever potentially troublesome statements such as the one in the example above are found. 


\section{REFERENCES}

1. J. T. Hogan, Multi-Fluid Tokamak Transport ModeZs, ORNL/TM-5153 (November 1975); Methods of Computational Physics, Vol: 16: Computer Applications to Controlled Thermonuclear Research, ed. by J. Killeen, B. Alder, and S. Fernbach (1976).

2. J. T. Hogan, Physical Models in the Oak Ridge Tokamak Transport Code, ORNL/TM, to he published.

3. H. Grad, P. N. Hu, and D. C. Stoveno, Proo, Natz. Aceds. Sreri. IISA, 72: 3789 (1975).

4. H. K. Meier, W. I. van Rij, C. 0. Beasley, Jr., and J. E. McCune, The ColZisional Plasma Model: A Velocity-Space Orthogonal-Function Representation for the Distribution Function of a Collisional Plasma, ORNL/TM-5314 (March 1976); H. K. Meier, W. I. van Rij, and C. O. Beasley, Jr., Techniques for Computing the Nrmerical. Valubs of tho Matrix Elements of the ColZision Operator in the Colzisional Plasma Model, ORNL/TM-5315 (March 1976); W. I. van Rij, H. K. Meier, C. O. Beasley, Jr., and J. E. McCune, Kinetic Equations for the Colzi3ional Plasma Model, ORNL/TM-5316 (March 1976); C. 0. Beasley, Jr., J. E. McCune, H. K. Meier, and W. I. van Rij, Numerical Study of Urift-Kinetic Evolution of ColZisional PLasmas in Tori, ORNL/IM-5317 (March 1976).

5. H. P. Furth, P. H. Rutherford, and H. Selberg, Phys. Fluids 16: 1054 (197.3). 


\section{DESCRIPTION OF MAIN PROGRAM MODULE AND SUPPORTING SUBROUTINES}

\subsection{OVERVIEW OF CODE STRUCTURE}

The module MAIN exercises primary program control, initializes all variables and arrays, computes plasma power balance at each time step of the simulation, generates a history of the plasma evolution, and contains most of the program output statements. A diagram of this module is shown in Fig. 1 .

The subroutine call statements in the basic time loop of the main program have been limited as much as possible to the basic modules used for the simulation. Switches used to select a particular option have been left in each module rather than put in the main program. In some ways this obscures. the logic in the program, since each module must be consulted in order to learn what logic and conventions are used. However, this program structure facilitates adding or changing modules. When module additions or changes are introduced, provision should be made to read in data and supervise variable and array initializations in GETSET, and to handle all output in subroutine PRTØUT (by use of an alternate entry point). Data transfer between GETSET, module, and PRT $\emptyset \mathrm{UT}$ can be made using a labeled common, unless a great deal of data is involved. Appendix II contains tables listing the variables and arrays with their definitions to aid the user who wants to add a new module or mudify an existing one.

Almost all output is handled by subroutine PRT $\emptyset \mathrm{UT}$, through the use of alternate entry points. This arrangement was chosen because of the economy of being able to use a few basic format statements to print the same sets of arrays at different stages of the simulation merely by inserting a call to the appropriate entry point name. This subroutine is never called ás such.

\subsection{INITIALIZATION PROCEDURE}

All initializations of variables, arrays, and machine parameters are handled in subroutine GETSEI, using the NAMELISI feature of FØRTRAN IV. 
ORNL- DWG 76-2304

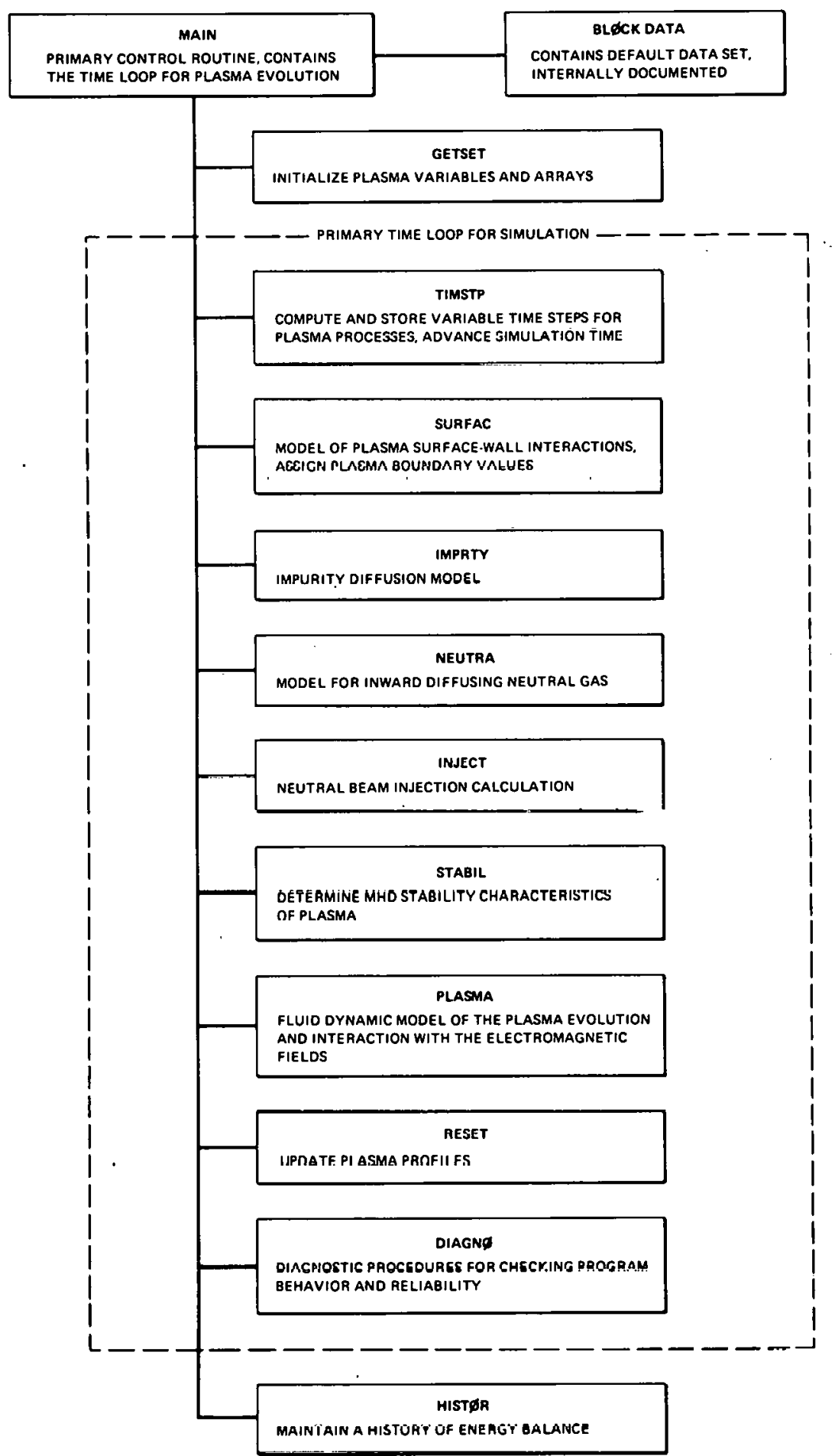

Fig. 1. Diagram of MAIN module and subroutines called. 
NAMELIST permits the user to include helpful documentation in the input data set without interfering in any way with the process of reading in the data set.

\subsection{BALANCES AND HISTORIES}

The plasma power and energy balances, comparisons between contributions of various terms in these balances, and the histories of selected plasma variables are calculated in subroutine $\emptyset \mathrm{RMPWR}$. Time histories are generated in the following arrays:

\begin{tabular}{cl} 
Array Name & Physical Process Contributing to Energy History \\
\cline { 2 - 3 } B1 & ohmic heating \\
B2 & thermal conduction and convection of electrons \\
B3 & thermal conduction and convection of ions \\
B4 & line radiation \\
B5 & energy loss due to charge exchange \\
B6 & stored energy of electrons \\
B7 & stored energy of ions \\
B8 & electron energy input from neutral beam injection \\
B9 & ion energy input from neutral beam injection
\end{tabular}

Power spatial integrals and fluxes are generated in the following arrays for the time intervals for which printout is specified:

\begin{tabular}{cl} 
Array Name & \multicolumn{1}{c}{ Associated Physical Piocess } \\
\cline { 2 - 3 } C1 & ohmic heating, integral over space \\
C2 & electron thermal conduction, flux \\
C3 & electron convection, flux \\
C4 & electron radiation loss; integral of line, recom- \\
& bination, and synchrotron radiation \\
C5 & electron-ion transfer for electrons, integral \\
C6 & ion thermal conduction, flux \\
C7 & ion convection, flux \\
C8 & energy loss due to charge exchange, integral \\
C9 & electron energy due to neutral beam injection, \\
& integral
\end{tabular}


C10

ion energy due to neutral beam injection, integral

C11

ionization loss due to electrons, integral

C12

electron-ion transfer for ions (proton), integral

\subsection{GRAPHICS OPTIONS}

No graphics options are available in the plasma simulation code per se. The graphics programs which have been developed at ORNL assume that a simulation run has been made and that the output has been written into a particular file or data set. Two forms of graphic display have been used: the graphics package $(\varnothing \mathrm{RGRAPH})^{1}$ developed for use at ORNL with the IBM 360 computers, and several interactive programs developed for use with the Tektronix 4000 series $^{2}$ storage tube terminal and the Tektronix Advanced Graphics ${ }^{3}$ software package. The code's output format reflects the use of the Tektronix 4000 series terminal and its associated hard copy producer.

\subsection{DESCRIPTIONS OF SUBROUTINES}

Each subroutine and function will be described, wherever possible, according to the following format:

Purpose or use

Equations used

Called Irom:

Subroutines called:

Commons required:

NAMELIST names:

Variables and arrays (output) changed:

Significant internal variables:

Limitations on variables:

Limitations in subroutine applications:

ROUTINE MAIN

The routine MAIN only calls subroutines; it does no direct computation. It contains the main time loop for the simulation, and calls for the final printout of plasma histories at the end of the simulation run. Subroutines called: GETSET, TIMSTP, NEUTRA, STABIL, SURFAC, PLASMA, RESET, DISGN $\emptyset$, EMPRTY, INJECT, HIST $\varnothing R$

Commons required: INDEX 
SUBRØUTINE GETSET

Subroutine GETSET reads in all the input data, initializes the variables and arrays used by each module in the simulation, and prints a report of the program input. The initializations of quantities for the various modules are made by calls to the executive subroutines for each module in the same order in which the data is read. This structure should make the initialization procedure more obvious. A set of default input data is passed from subprogram BL $\varnothing \mathrm{CK}$ DATA into this subroutine through labeled common blocks, so that the program can be run as soon as it is implemented on a particular computer. The documentation of the input data internal to BL $\varnothing \mathrm{CK}$ DATA was taken directly from an input data set in which the NAMELIST list of data appeared where the default values are set in DATA statements.

Two options exist for initializing the plasma variable profiles. The choice of option is made with the switch $\operatorname{INIT(1).~If~} \operatorname{INIT(1)}=0$, the following analytic expressions are used (see ENTRY IPPC in executive subroutine PLASIM of the plasma module):

(i) $j_{Z}(r)= \begin{cases}Z J \emptyset\left[1-\left(\frac{r}{R 9}\right)^{2}\right] X I(1), & R<R 9<A M \\ 0.001 & , A M \geqslant R \geqslant R 9\end{cases}$

(ii) $T_{e}(r)= \begin{cases}(\mathrm{TE} \emptyset-\mathrm{TEB})\left[1-\left(\frac{\mathrm{r}}{\mathrm{R} 9}\right)^{2}\right] \mathrm{XI}(2)+\mathrm{TEB}, & \mathrm{R} \leqslant \mathrm{R} 9<\mathrm{AM} \\ \mathrm{TEB} & , \text { AM } \geqslant R \geqslant R 9\end{cases}$

(iii) $T_{i}(r)= \begin{cases}(\mathrm{TI} \emptyset-\mathrm{TEB})\left[1-\left(\frac{\mathrm{r}}{\mathrm{R} 9}\right)^{2}\right] \mathrm{XI}(3)+\mathrm{TIB}, & \mathrm{R} \leqslant \mathrm{R} 9 \leqslant \mathrm{AM} \\ \mathrm{TIB} & , \mathrm{AM} \geqslant \mathrm{R} \geqslant \mathrm{R} 9\end{cases}$ 


$$
\begin{aligned}
& \text { (iv) } \mathrm{N}_{\mathrm{e}}(\mathrm{r})= \begin{cases}(\mathrm{DEN} \emptyset-\mathrm{DENB})\left[1-\left(\frac{r}{\mathrm{R} 9}\right)^{2}\right] \mathrm{XI}(4)+\mathrm{DENB} & , 0 \leqslant \mathrm{R} \leqslant \mathrm{R} 9 \\
\mathrm{DENB} & , \mathrm{R} 9 \leqslant \mathrm{R} \leqslant \mathrm{AM}\end{cases} \\
& \text { (v) } \begin{cases}\mathrm{B}_{\mathrm{p}}=0 & \\
\mathrm{~B}_{\mathrm{p}}=2 \mathrm{~J} \emptyset\left(\frac{0.2}{\mathrm{r}}\right)\left\{1-\left[1-\left(\frac{r}{\mathrm{R} 9}\right)^{2}\right] X I(1)+1\right\}, & 0 \leqslant \mathrm{r} \leqslant 9 \\
\mathrm{~B}_{\mathrm{p}}=\mathrm{B}_{\mathrm{p}}(\mathrm{r}-\Delta \mathrm{r}) *(\mathrm{r}-\Delta \mathrm{r}) / \mathrm{r}, \mathrm{r} 9 \leqslant \mathrm{r} \leqslant \mathrm{AM} & \end{cases}
\end{aligned}
$$

After an initial call to compute diffusion coefficients (subroutine CDC) the following equations are used:

$$
\begin{aligned}
& \text { (vi) } E(x)=n(x) \cdot f(r) \\
& \text { (vii) } V(0)=0 \\
& V(r>0)=-T^{*}(\mathrm{dN} / \mathrm{dr}) / \mathrm{N}
\end{aligned}
$$

If INIT(1) > 0 , initial profiles are read in from cards, according to the format $(2 \mathrm{~F} 10.0,2 \mathrm{~F} 10.3,4 \mathrm{PE} 10.2)$. The list of variables, for $1 \leqslant I \leqslant N$, is 38 follows:

$$
\begin{aligned}
& \text { TE (I) - electron temperature, eV } \\
& \mathrm{IT}(\mathrm{I}) \text { - ion temperature, eV } \\
& \mathrm{ZJ}(\mathrm{I}) \text { - plasma toroidal current density, } \mathrm{a} / \mathrm{cm}^{2} \\
& B(I) \text { - poloidal magnetic field, G } \\
& \text { E(I) - electric field (toroidal), } \mathrm{V} / \mathrm{cm} \\
& \text { DEN(I) - plasma electron density, } \mathrm{cm}^{-3} \\
& \text { VEL (I) - plasma ion drift velocity, } \mathrm{em} / 3 c \mathrm{c} \\
& \mathrm{ZN} 1(1) \text { - total neurral gas dens1ty, } \mathrm{cm}^{-3}
\end{aligned}
$$

These variables are also the ones printed out in several of the profile summaries in subroutine PRTØUT. The variables can be punched out on cards to be read at this point for restarting or continuing a simulation run, if time limitations or other considerations require doing the simulation piecemeal. If the option of reading these profiles is used, the - profile data must follow the data read by the NAMELIST feature. 
The initial profiles required for variables associated with the neutral gas, impurity, and neutral beam injection modules will be generated by calls to the executive subroutines which maintain control over the computations done by each module. The arrays which must be initialized are:

1. Neutral gas module:

$T \emptyset$ - neutral gas temperature profile

ZN1 - total neutral gas density

2. Impurity module:

DENP - ion density profile

D1 - spatial profile of effective ionic charge; D1 > 1 with impurities

$\mathrm{HIZ}$ - radiation from high-Z impurities

QRD - radiation from low $Z$ impurities

ZBR $-Z$ bracket $[\mathrm{Z}]$, defined by:

$$
[\mathrm{z}] \equiv \frac{\sum_{\text {species } \mathrm{k}_{\mathrm{k}} \mathrm{m}_{\mathrm{proton}}} \mathrm{n}_{\mathrm{k}} \mathrm{z}_{\mathrm{k}}^{2}}{\sum_{\text {species } \mathrm{k} \mathrm{n}_{\mathrm{k}} \mathrm{z}_{\mathrm{k}}}}
$$

CARB $\emptyset \mathrm{N}$ - total density of carbon impurity, $\mathrm{cm}^{-3}$

$\emptyset$ XYGEN - total density of oxygen impurity, $\mathrm{cm}^{-3}$

$\mathrm{CC}(\mathrm{K}, \mathrm{I})$ - density of $(\mathrm{K}-\mathrm{I})$ th charge state of carbon at I-th grid point

$C \emptyset(K, I)$ - density of $(K-1)$ th charge state of oxygen at I-th grid point

3. Neutral beam injection module:

QE1 - injected neutral beam heat input to electrons

QI1 - injected neutral beam heat input to ions

IJT - fast ion current produced by injected neutral beam

Table 2 contains a l1st of the labeled common blocks used by GETSET, together with lists of the input and/or output variable and array names.

Called from:

Subroutines called: Commons required:
MAIN

CDC, NEUTRA, IMPRTY, BMSTRT, INIØUT

BDYC $\emptyset \mathrm{N}, \mathrm{BEAMC}, \mathrm{CDCLBX}$, CURENT, ELCTRN, FIELDS, GEøM, IMPURT, INDEX, I $\emptyset \mathrm{NS}$, NEUTRL, NEWS, TEMP, TIME, TMINDX, XPфRT, ZRAD 
Namelist names:

MACHIN, TIMINT, PLADAT, NEGADA, IMPIDA, NBMIDA, IØDATA, TEMPDA

Input:

See Section 1.5

Variab1es changed:

See Table 2 and Appendix II. 
Table 2. List of labeled C $\emptyset \mathrm{MM} \emptyset \mathrm{N}$ blocks and elements in them used by subroutine GETSET

\begin{tabular}{|c|c|c|c|c|}
\hline \multirow[b]{2}{*}{$\mathrm{C} \emptyset \mathrm{MM} \emptyset \mathrm{N} \quad \mathrm{BL} \emptyset \mathrm{CK}$} & \multicolumn{2}{|c|}{ Input } & \multicolumn{2}{|c|}{ Output } \\
\hline & Variables & Arrays & Variables & Arrays \\
\hline$/ \mathrm{BDYC} \varnothing \mathrm{N} /$ & $\begin{array}{l}\text { DENB, DEN } \emptyset, \text { TEB } \\
\text { TE } \emptyset, \text { TI } \emptyset\end{array}$ & & & \\
\hline /BEAMC/ & $\begin{array}{l}\text { DTCH, DTCGL, RC, } \\
\text { RB, BHLF, TNJCT }\end{array}$ & CURBM, ENGY, INJ & . & DJT \\
\hline /CURENT/ & $\mathrm{ZFI}, \mathrm{ZPC}$ & & & \\
\hline /ELCTRN/ & $\mathrm{FQE}$ & & & DEN \\
\hline /FIELDS/ & BT & & $\mathrm{ZJ} \emptyset$ & $B, E, Z J$ \\
\hline /GE $\emptyset \mathrm{M} /$ & $A M, R \emptyset, R 9$ & & AREA, HR, VøL & \\
\hline iIMPURT / & $\begin{array}{l}\text { PCIMP, } X \varnothing X, X C A R \text {, } \\
\text { ZEFF, ZIMP }\end{array}$ & & & $\begin{array}{c}\text { CARB } \emptyset \mathrm{N}, \emptyset \mathrm{XYGEN}, \mathrm{CC}, \\
\mathrm{C} \emptyset, \mathrm{DENP}, \mathrm{ZBR}\end{array}$ \\
\hline iINDEX/ & $\mathrm{N}, \mathrm{NT}, \mathrm{NR}$ & NUTE, IMP, INIT & TIC, NM1 & \\
\hline$i \mathrm{I} \emptyset \mathrm{NS} /$ & AMU & & & DEN \\
\hline /NEUTRL/ & $\begin{array}{l}\text { ATT, FCDEN, FCF, } \\
\text { PINJ }\end{array}$ & & & QEI QI1, ŻN1 \\
\hline /NEWS/ & & & NEWS & \\
\hline /TEMP/ & & & & $\mathrm{TE}, \mathrm{TI}, \mathrm{T} \emptyset, \mathrm{VEL}$ \\
\hline$/ \mathrm{T} \operatorname{IME} /$ & $\begin{array}{l}\text { TC, TSTAR, TMAX, } \\
\text { TP } \emptyset\end{array}$ & & HT, TIM, TPR & \\
\hline /TMINDX/ & DTNJCT, N1, N3 & & , & . \\
\hline /XPøRT/ & & D11 & & D1 \\
\hline /ZRAD/ & & & & $\mathrm{HIZ}, \mathrm{QRD}$ \\
\hline
\end{tabular}


SUBR $\emptyset \mathrm{UTINE} \emptyset \mathrm{RMPWR}$

Subroutine $\emptyset$ RMPWR computes the plasma gross power balance, particle and energy confinement times, fluxes through surfaces, plasma volume sources and sinks, and a series of terms contributing to the gross power balance. This subroutine is the primary interface between the plasma simulation code and the user. Table 3 summarizes the basic physical processes considered and gives the names of the variables and arrays associated with them.

The follnwing types ot quantities are calculated:

1. Amount of power associated with the dominant plaswa processes

2. Total amount of energy involved with each domlnall prusess

3. Fluxes through the plasma surface

4. Confinement times

5. Safety factor

6. Number of microwave interferometer fringe shifts resulting from changes in the plasma density

7. Ratios of various plasma power loss terms to the power put into the plasma.

Section 2.3 gives the definitions of the $B$ and $C$ series arrays which are generated in $\emptyset \mathrm{RMPWR}$. 'l'he $B$ seriès array $1 \mathrm{~s}$ gelletaled in the section of this subruutine defined by the alternate entry SAVPWR, which is executed at each time step. Time histories of quantities in the $B$ series arrays are stored for 100 equal time intervals which span the entire duration of the simulated plasma discharge. The B serics arrays are printed at the end of the simulation run.

The $C$ series arrays are generated in the section defined by the alternate entry NEWPWR, which is executed only for those time intervals for which printout of the plasima profiles has bcon epectified. A summary of plasma variable values at the plasma center is given for the course of the simulation in the array RNEWS.

Table 4 shows which elements in the 1 abeled common blocks supply information as input and which elements are defined or altered for output. 
Table 3. Physical processes and names of variables and arrays associated with them in subroutine $\emptyset \mathrm{RMPWR}$

\begin{tabular}{|c|c|c|c|c|c|c|c|}
\hline Process & $\begin{array}{l}\text { Contribution } \\
\text { to Plasma } \\
\text { Power }\end{array}$ & $\begin{array}{l}\text { Total Amount } \\
\text { of Energy } \\
\text { Involved } \\
\text { Time Integral } \\
\text { of Power }\end{array}$ & $\begin{array}{l}\text { Flux } \\
\text { Profiles }\end{array}$ & $\begin{array}{l}\text { Flux } \\
\text { Through } \\
\text { Plasma } \\
\text { Outer } \\
\text { Boundary }\end{array}$ & $\begin{array}{l}\text { Power } \\
\text { Flow } \\
\text { Through- } \\
\text { out } \\
\text { Plasma }\end{array}$ & $\begin{array}{l}\text { Values of } \\
\text { Integrals } \\
\text { of Profiles }\end{array}$ & $\begin{array}{c}\text { Power } \\
\text { Densities }\end{array}$ \\
\hline Ohmic heating & $\mathrm{P} \emptyset \mathrm{H}$ & $\mathrm{P} \emptyset \mathrm{X}, \mathrm{B} 1$ & & & $\mathrm{C} 1$ & SUM $\emptyset$ & PIN \\
\hline $\begin{array}{l}\text { Poloidal magnetic } \\
\text { field }\end{array}$ & & & & & & SUMBT & \\
\hline Line radiation & PLR & PLRX, B4 & & & & SUMLR & \\
\hline $\begin{array}{l}\text { Electron-ion } \\
\text { collisions }\end{array}$ & & & & & & SUMEI & \\
\hline (For electrons on & & & & & & & \\
\hline $\begin{array}{l}\text { Electron thermal } \\
\text { conduction }\end{array}$ & & & PED B2 & PEX, B2 & & & ETE \\
\hline Electron convection & & & PCVE, C3 & & & & EB \\
\hline $\begin{array}{l}\text { Electron energy, } \\
\text { internal }\end{array}$ & ZE, B6 & & & & & $\begin{array}{l}\text { SUMEX, } \\
\text { SUMP } a\end{array}$ & \\
\hline $\begin{array}{l}\text { Electron energy, } \\
\text { neutŕal beam } \\
\text { injection }\end{array}$ & PER & $\mathrm{PHE}, \mathrm{B} 8$ & & & $\mathrm{Cg}$ & SNJE & $\mathrm{EE} \emptyset$ \\
\hline $\begin{array}{l}\text { Ionization loss due } \\
\text { to electrons }\end{array}$ & PEIL & & & & $\mathrm{Cl1}$ & SUMQE & $\mathrm{PE} \emptyset$ \\
\hline
\end{tabular}


Table ? (continued)

\begin{tabular}{|c|c|c|c|c|c|c|c|}
\hline Process & $\begin{array}{l}\text { Contribution } \\
\text { to Plasma } \\
\text { Fower }\end{array}$ & $\begin{array}{l}\text { Total Amount } \\
\text { of Energy } \\
\text { Involved } \\
\text { Time Entegra- } \\
\text { of Power }\end{array}$ & $\begin{array}{l}\text { Flux } \\
\text { Profiles }\end{array}$ & $\begin{array}{l}\text { Flux } \\
\text { Through } \\
\text { Plasma } \\
\text { Outer } \\
\text { Boundary }\end{array}$ & $\begin{array}{c}\text { Fower } \\
\text { Flow } \\
\text { Through- } \\
\text { out } \\
\text { Plasma }\end{array}$ & $\begin{array}{l}\text { Values of } \\
\text { Integrals } \\
\text { of Profiles }\end{array}$ & $\begin{array}{l}\text { Power } \\
\text { Densities }\end{array}$ \\
\hline Electron-Zon transfer & PEI & & & & 55 & & $E I \emptyset$ \\
\hline $\begin{array}{l}\text { Synchrotron } \\
\text { bremsst:ahlung } \\
\text { (For protons only) }\end{array}$ & PLR & & & . & $C 4$ & & SHR \\
\hline $\begin{array}{l}\text { Ion thermal } \\
\text { conduction }\end{array}$ & & & $\mathrm{PID}, \mathrm{C} 6$ & PIX, B? & & & ETI \\
\hline Ion convection & & & PCIV, C7 & & & & $\mathrm{PB}$ \\
\hline Ion energy, internal & ZSI, B? & & & & & SUMIX, SUZI & \\
\hline $\begin{array}{l}\text { Ion energy, neutral } \\
\text { beam injection }\end{array}$ & PIR & PHI, B9 & & & C10 & SNJI & EE1 \\
\hline $\begin{array}{l}\text { Ionizaticn loss due } \\
\text { to charge exchange }\end{array}$ & PIIL & PCXX, B5 & & & $\mathrm{C8}$ & SUMQI & $P I \emptyset, P C X$ \\
\hline Ion-electron transfer & FIE & & & & $\mathrm{c12}$ & & EII \\
\hline
\end{tabular}

$a_{\text {SUMP }}$ is also used as a cifferent type o quantity in.computation of gross energy confinement time. 
Table 4. List of labeled commons and elements within them used by subroutine $\emptyset$ RMPWR

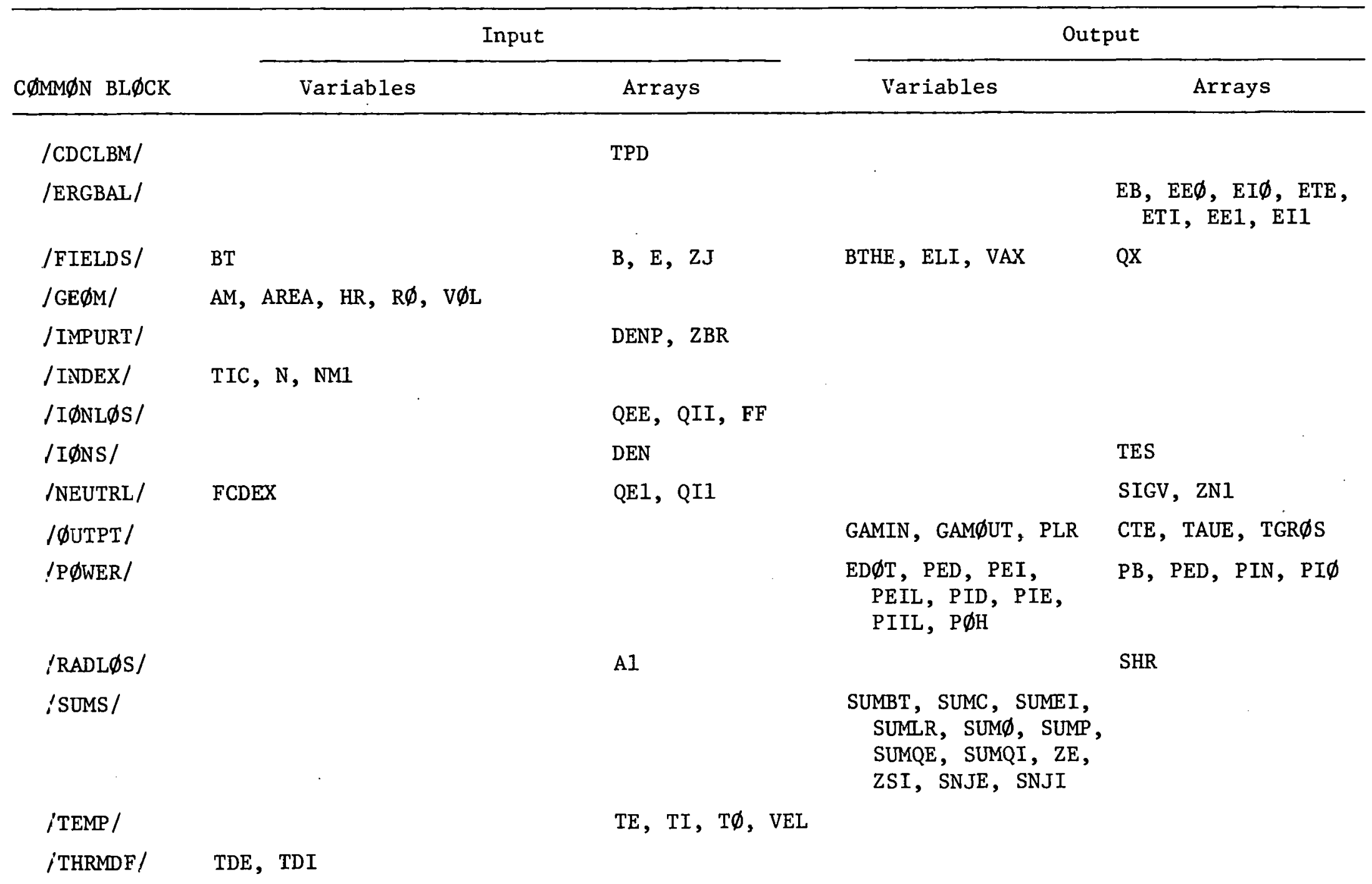


Table 4 icontinued)

\begin{tabular}{|c|c|c|c|c|c|}
\hline \multirow[b]{2}{*}{$\mathrm{C} \varnothing \mathrm{MM} \emptyset \mathrm{N} \quad \mathrm{BL} \emptyset \mathrm{CK}$} & \multicolumn{3}{|c|}{ Input } & \multicolumn{2}{|c|}{ Output } \\
\hline & Variatles & & Arrays & Variables & Arrays \\
\hline /TIME/ & HT, TITRT, TIM, & TMAX & & & \\
\hline /XPøRT/ & & & $\mathrm{D} 1, \mathrm{D} 6$ & & \\
\hline /XTRA/ & & & $\mathrm{ALF}$ & & $\mathbf{F}$ \\
\hline /ZRAD/ & & & $\mathrm{QRD}$ & & \\
\hline /CXLØs/ & & & $\mathrm{QCX}$ & & . \\
\hline /HIST/ & & & & , & $\begin{array}{c}\mathrm{B} 1, \mathrm{~B} 2, \mathrm{~B} 3, \mathrm{~B} 4, \mathrm{~B} 5 \\
\mathrm{~B} 6, \mathrm{~B} 7, \mathrm{~B} 8, \mathrm{~B} 9\end{array}$ \\
\hline /HISTI/ & & & & $\begin{array}{l}\text { PøX, PEX, PIX, } \\
\text { PLRX, PCXX, } \\
\text { SUMEY, SLMLX, } \\
\text { PHE, PHI, ICM }\end{array}$ & \\
\hline /RATI $\emptyset$ / & & & & $\begin{array}{l}\text { PCVE, PCVI, KCUR, } \\
\text { RCVE. RCII, TAX, } \\
\text { TAXZ, TAXI }\end{array}$ & \\
\hline /PINT/ & & & & & $\begin{array}{l}\mathrm{C} 1, \mathrm{C} 2, \mathrm{C} 3, \mathrm{C} 4, \mathrm{C} 5, \\
\mathrm{C} 6, \mathrm{C} 7, \mathrm{C} 8, \mathrm{C} 9, \\
\mathrm{C} 10, \mathrm{Cl}, \mathrm{C} 12\end{array}$ \\
\hline /RATI $\emptyset 1 /$ & & & & $\begin{array}{l}\text { REI, RTH, RPLR, } \\
\text { RD } \emptyset \mathrm{T} \text {, RTHE, RTHI }\end{array}$ & \\
\hline$/ \mathrm{CXL} \emptyset \mathrm{s} /$ & & & & & PCX \\
\hline /NEWS/ & & & & NEWS & RNEWS \\
\hline
\end{tabular}


Called from:

RESET

Subroutines called: PC

Commons required:

CDCLBM, CURENT, CXL $\emptyset S$, ERGBAL, FIELDS, GE $\emptyset \mathrm{M}$, HIST, HIST1, IMPURT, INDEX, IØNL $\emptyset S$, NEWS, RATI $\varnothing S$, RATI $\emptyset 1$, SUMS, TEMP, THRMDF, TIME, XP. RRT $_{\text {, XTRA, }}$ ZRAD

Variables required: IT. See also Table 3 and Appendix II.

Variablés changed: See Tables 3 and 5 and Appendix II. In addition, there are:

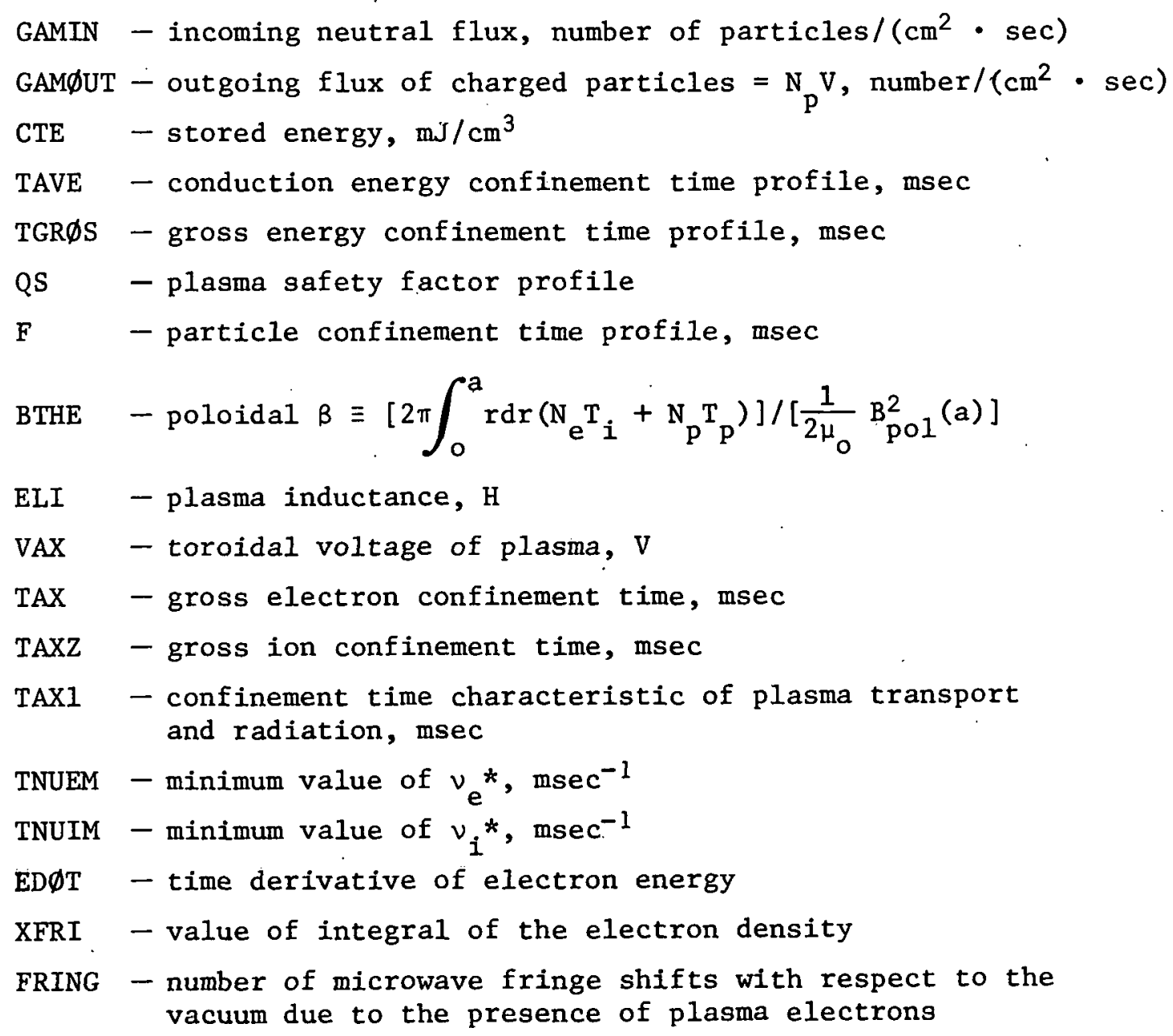


Table 5. Variables representing ratios of power transported by various physical processes to total power input (from ohmic heating plus neutral beam injection)

\begin{tabular}{ll}
\hline $\begin{array}{c}\text { Ratio } \\
\text { Variable }\end{array}$ & \multicolumn{1}{c}{ Physical Process } \\
RCVE & $\begin{array}{l}\text { Electron convection } \\
\text { Ion (proton) convection } \\
\text { RCVI }\end{array}$ \\
REI & Electron-ion transfer \\
RTH & Total thermal conduction \\
RPLR & Line radiation \\
KUW'I & ElecLlun hudiug or cooling \\
RTHE & Electron thermal conduction \\
RTII & Ion (proton) convection
\end{tabular}




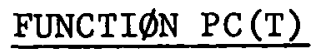

Function PC(T) generates the program current, i.e. the driving current for the plasma. It is a function constructed to fit the driving current that actually produces the plasma discharge. The form of the function used in this subroutine is:

$$
\mathrm{PC}=\mathrm{ZPC}+\mathrm{ZFI*}(1 \cdot-\operatorname{EXP}(-\mathrm{T} / \mathrm{TC})),
$$

where

$$
\begin{aligned}
\mathrm{ZPC} & =\text { initial value of the total current } \\
\mathrm{ZFI} & =\text { additional amount of total current after time } \mathrm{TC} \\
\mathrm{T} & =\text { value of current elapsed simulation time } \\
\mathrm{TC} & =\text { rise time of the current }
\end{aligned}
$$

Called from: $\quad$ RMPWR, LBM

Commons required: CURENT, TIME

Variables required: $T$

Variables changed: PC (the function name) 


\section{SUBRØUTINE PRTØUT}

Subroutine PRTøUT handles only output from the simulation calculations. No calculations are done in this subroutine. Use of alternate entry points puts various format statements at the disposal of the user so the statements can be used any number of times for printing out different arrays. This organization also permits the user merely to insert a call to an alternate entry point in any subroutine he chooses, thus gaining access to several write and format statements by inserting a single line when output 1s destred.

The alternate entries and the subroutines calling them are:

\begin{tabular}{cl} 
Alteinate Entry & Called From \\
\cline { 2 - 2 } INI $\emptyset \mathrm{UT}$ & GETSET \\
PR $\emptyset \mathrm{FIL}$ & RESET \\
SUMARY & RESET \\
NUPRIN & NEUTRA \\
IMPRIN & IMPRTY \\
HIST $\emptyset \mathrm{R}$ & MAIN
\end{tabular}

Commons required: C $\emptyset \mathrm{UNT}, \mathrm{CXL} \emptyset \mathrm{S}$, ERGBAL, FIELDS, HIST, IMPURT, INDEX, IØNS, MEASUR, INEUTRL, NEWS, ØUTPT, PINT, FØWER,

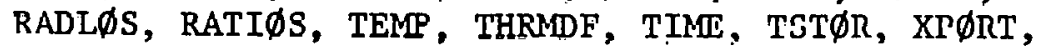
XTRA, ZRAD

Quantities printed:

\begin{tabular}{|c|c|}
\hline & 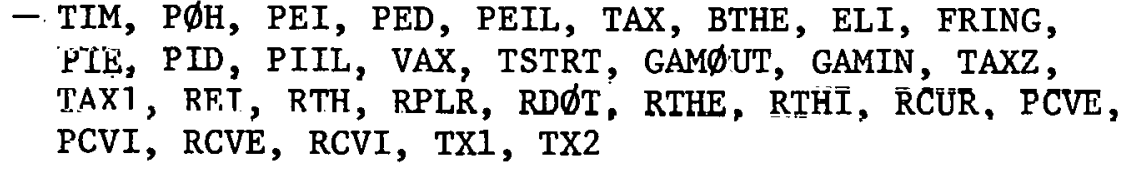 \\
\hline Arrays & 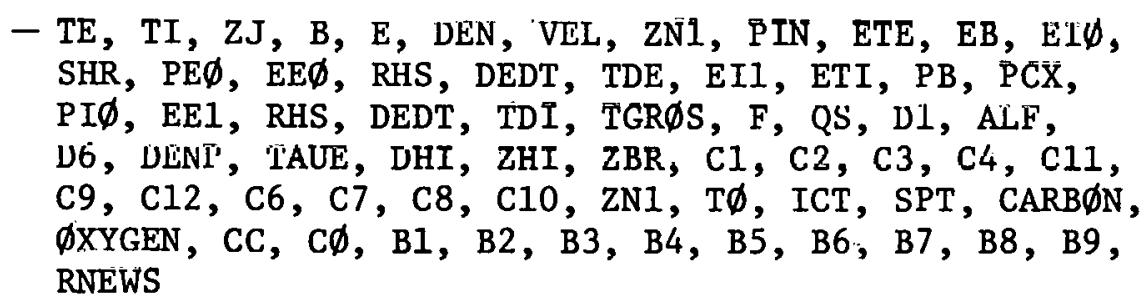 \\
\hline
\end{tabular}


SUBRØUTINE RESET $(I T, K, *)$

Subroutine RESET uses the solutions generated by the PLASMA module to set the new values of the plasma variable profiles, and to compute the new values of ionization loss. It also prints the new profiles, calls for the power balance calculations, and prints a summary of the power balance. Figure 2 shows the order of calls to other subroutines and the logic of the time sequence which is observed by RESET.

The electron and ion temperature and plasma density profiles are checked at each time step to be sure they are in a reasonable range of physical values. If the values go out of range, an attempt is made to recover by force. The profiles are set to their edge boundary values from the radial point where trouble occurs on out to the plasma edge.

Called from: MAIN

Subroutines called: SAVPWR, PRØFIL, BMPWR, NEWPWR, SUMARY Commons required: $\quad$ BDYC $\emptyset \mathrm{N}$, BEAMC, FIELDS, GE $\emptyset \mathrm{M}$, IBTMN, INDEX, I $\emptyset \mathrm{NS}$, MEASUR, NEUTRL, RADL $\emptyset$ S, TEMP, TIME, XTRA, ZRAD

Variables required:

$$
\begin{aligned}
& \text { IT - } \quad-D \emptyset \text { loop variable in the main program for number } \\
& \text { /BDYCON/ - DENB, TE } \emptyset, T I \emptyset \\
& \text { /BEAMC/ - DJT(101), TNJCT } \\
& / G E \emptyset \mathrm{M} / \quad-\mathrm{HR} \\
& \text { /IBTMN/ }-\mathrm{X}(360) \\
& \text { /INDEX/ }-\mathrm{N}, \mathrm{NR} \\
& \text { /I ØNS/ - DEN(101) } \\
& \text { /TIME/ - HT, TIM, TMAX, TPR }
\end{aligned}
$$

Variables and arrays changed:

$$
\begin{array}{ll}
\text { Single variables } & - \text { XFRI, TPR } \\
\text { Arrays } & - \text { TE, TI, BZ, DEN, A3, ZJ, B, E, VEL, DJE, TE12. } \\
& \text { See APpendix II. } \\
\text { A3 } & - \text { time derivative of the electron density }
\end{array}
$$


ORNL-DWG $76-2316$

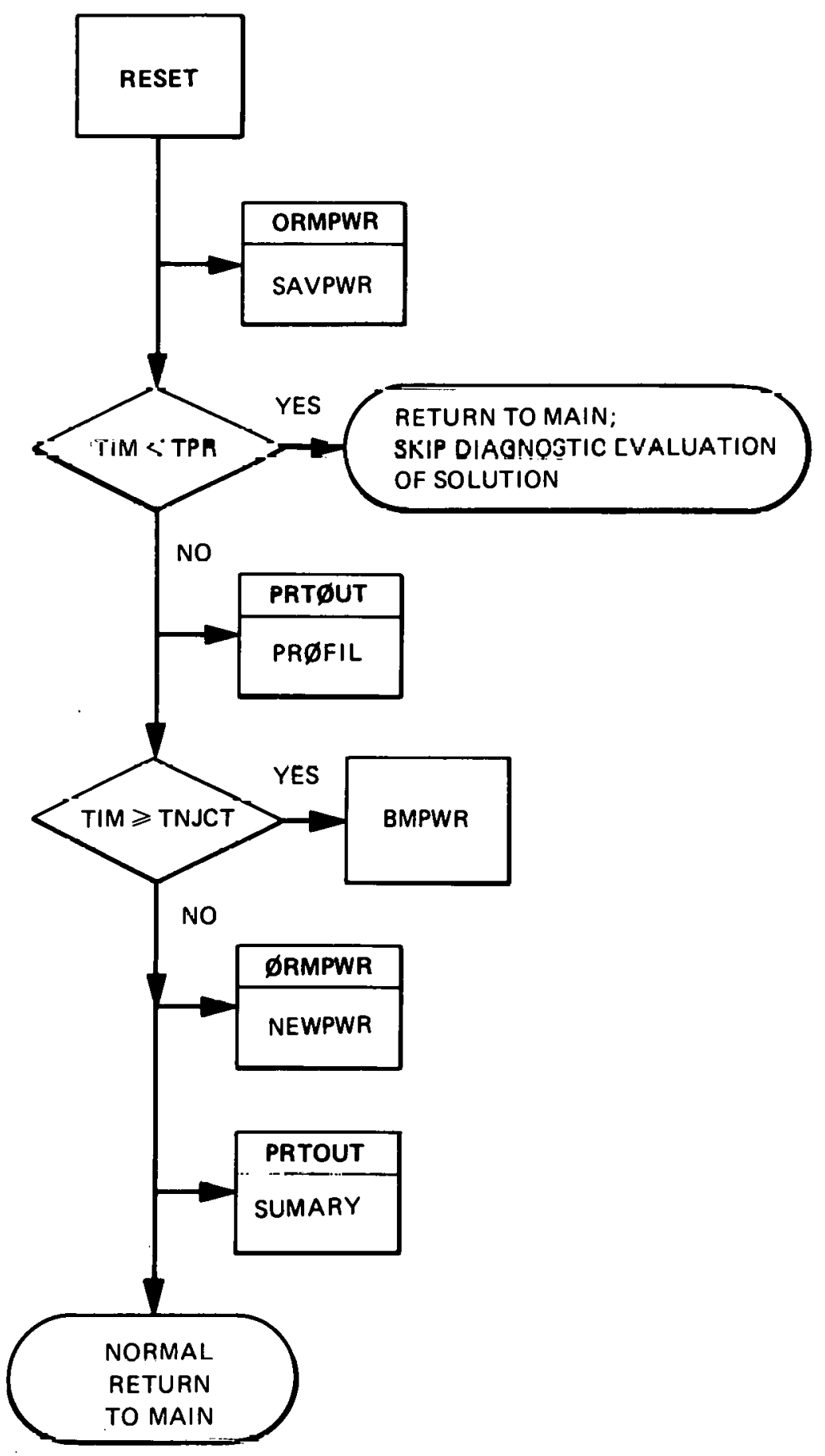

Fig. 2. Diagram of subroutine calls and logic of subroutine RESET. 
SUBRØUTINE SIGMA (ZEO, ZTE, ZSIGCK, ZSIGI, ZSIGEL, NS)

Subroutine SIGMA calculates the cross sections for charge exchange $\sigma_{c x}$, ionization by ion impact $\sigma_{i i}$, and ionization by electron impact $\sigma_{i e}$. The cross sections for charge exchange and ionization by ion impact are taken from a paper by Riviere. ${ }^{4}$ Cross sections for $\mathrm{D}$ and $\mathrm{T}$ are obtained by using the energy/nucleon to compute the velocity. The expressions used in the subroutine are:

$$
\begin{aligned}
& \sigma_{c x}=\frac{6.937(10)^{-15}\left[1-0.155 \log _{10} \mathrm{~T}_{i}\right]^{2}}{1=1.112(10)^{-15} \mathrm{~T}_{i} 3.3} \mathrm{~cm}^{2}, \mathrm{~T}_{i} \text { in } \mathrm{eV} \\
& \log _{10} \sigma_{i .}=-0.8712\left(\log _{10} \mathrm{~T}_{i}\right)^{2}+8.156 \log _{10} \mathrm{~T}_{i}-34.833 .
\end{aligned}
$$

The rate equation formula for ionization by electron impact was derived by $R$. Colchin ${ }^{5}$ and is given by:

$$
\begin{aligned}
& \quad\left(3.97 \ln \frac{\mathrm{T}_{\mathrm{e}}}{10}-1.57\right) \frac{(10)^{-16}}{\mathrm{~T}_{\mathrm{e}}} \mathrm{cm}^{2}, \mathrm{~T}_{\mathrm{e}}<67.5 \mathrm{eV} \\
& \sigma_{\text {ie }}= \\
& \left(21.9 \ln \mathrm{T}_{\mathrm{e}}-42.9\right) \frac{(10)^{-16}}{\mathrm{~T}_{\mathrm{e}}} \mathrm{cm}^{2}, \mathrm{~T}_{\mathrm{e}} \geqslant 67.5 \mathrm{eV} .
\end{aligned}
$$

If the computed $\sigma_{i e}<0$, then $\sigma_{i e}=10^{-20} \mathrm{~cm}^{2}$.

C.alled from: CST

Variables required:

ZE $\varnothing$

- ion energy/nucleus, $\mathrm{eV}$

ZTE

NS

- electron energy, $\mathrm{eV}$
- switch with the following possible values:

$=1$, compute $\sigma_{\mathrm{cx}}$ only

$=2$, compute $\sigma_{i i}$ only

$=3$, compute $\sigma_{i e}$ only

$=4$, compute $\sigma_{c x}, \sigma_{i i}$, and $\sigma_{i e}$ 
Variables changed:

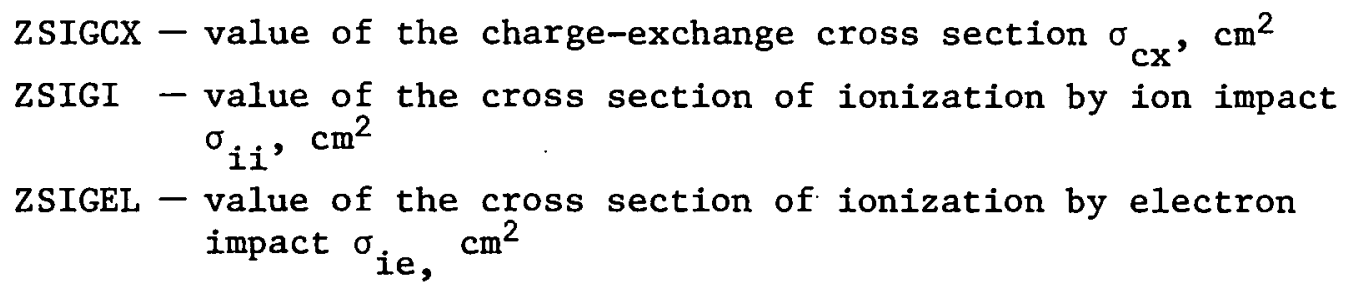

Limitations: The formulae given above represent fits to data and are valid in the following ranges:

$$
\begin{aligned}
& \sigma_{\mathrm{ex}}: 10 \mathrm{eV}<\mathrm{T}_{\mathrm{j}}<100 \mathrm{keV} \\
& \sigma_{i i}: 10 \mathrm{eV}<\mathrm{T}_{i}<100 \mathrm{keV} \\
& \sigma_{i e}: 20 \mathrm{eV}<\mathrm{T}_{c}<100 \mathrm{keV}
\end{aligned}
$$


SUBRØUTINE TIMSTP(IT)

Subroutine TIMSTP uses the timing information provided by the input to set up in a general way an array containing times when processes are turned on or off with respect to the beginning simulation time of the plasma discharge. The input switch arrays are used to select an initialization path for the timing and to determine the size of the time steps for the discharge. Small time increments can be chosen for the simulation calculation during periods of rapid changes due to fast current rise in the plasma and turn-on of neutral beam injection. The timing relationships are shown in Fig. 3.

Called from: $\quad$ MAIN

Commons required: BEAMC, INDEX, TMINDX, TIME

Variables required:

IT - D $\$$ loop variable in the main program for number of simulation time intervals that have elapsed

From labeled common blocks (see Append1x II for definitions)

$$
\begin{aligned}
& \text { /BEAMC/ - TNJCT, INJ(10) } \\
& \text { /INDEX/ - NT } \\
& \text { /TMINDX/ - DTNJCT, N1, N3 } \\
& \text { /TIME/ - TC, TSTRT, TMAX }
\end{aligned}
$$

Variables and arrays changed:

First time the subroutine is called, the arrays TT, NN, $\mathrm{HD}$.

First and all other times:

TIM - current value of elapsed time of the simulated plasma discharge, msec

HT - current time increment for the simulation, msec 

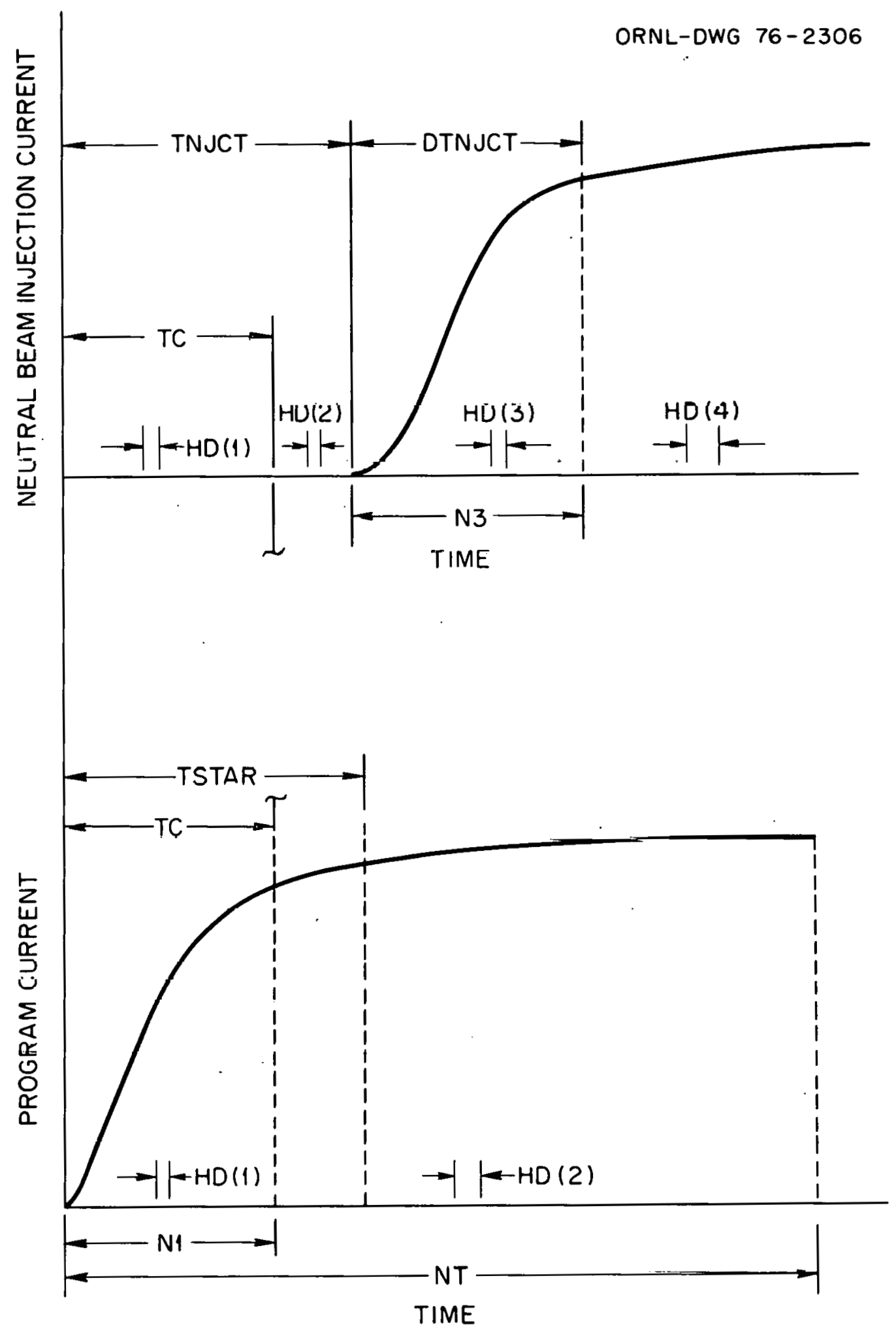

Fig. 3. Timing information used by plasma simulation code. 


\section{REFERENCES}

1. C. W. Nestor, Jr., Kay C. Chandler, N. B. Gove, and J. D. McDowel1, User's Manual for the Graphics Package ØRGRAPH, ORNL-4596 (November 1974); C. W. Nestor, Jr., Addendum to User's Manual for the Graphic Package ØRGRAPH, ORNL-4596 Addendum (January 1975).

2. "4010 and 4010-1 User's Manua1," Tektronix, Inc., P.0. Box 500, Beaverton, Oregon 97005.

3. "Terminal Control System V.3 User's Manual" and "Advanced Graphing II User's Manual," Information Display Division, Tektronix, Inc., P.O. Box 500, Beaverton, Oregon 97005.

4. A. C. Riviere, Nucl. Fusion 11: 363 (1971).

5. R. J. Colchin, Nucl. Fusion 11: 329 (1971). 


\section{THIS PAGE}

\section{WAS INTENTIONALLY \\ LEFT BLANK}




\section{DESCRIPTION OF PLASMA MODULE}

\subsection{PURPOSE AND STRUCTURE OF MODULE}

The plasma module contains the heart of the plasma simulation code. It is used to drive the evolution of the plasma with reference to the basic system of coupled partial differential equations describing the fluid model. This module makes no specific reference to plasma processes such as impurity diffusion effects, neutral gas transport, or the slowing down and trapping of an injected neutral beam. The influence of these processes on plasma evolution is provided through the rates of change of number, momentum, and energy of the fields and primary species which have been calculated in the pertinent module.

Subroutine CDC computes diffusion, particle, and energy conduction coefficients; resistivity; and associated derivatives with respect to the plasma variables. This information is then used to form the elements of the matrix needed to solve the system of equations. The matrix elements are computed and stored by subroutine LBM.' The solutions are obtained by subroutine. IBTM. The source terms and cross sections needed for the plasma model are computed by service subroutines CST and SIGMA, respectively.

The plasma module can be modified to include new physics or a different model for the plasma within the basic framework of the simulation code. The use of a different model for the diffusion coefficients will require changing some variables in subroutine $\mathrm{CDC}$ and perhaps making some corresponding changes in a few lines in subroutine LBM. The descriptions of the subroutines in this module are therefore written under the assumption that such changes will be made. The structure of the subroutines is presented in such a way as to aid the user in making his own changes.

\subsection{BASIC INPUT/OUTPUT}

The Input required by this module includes machine geometry, toroidal magnetic field, plasma ion mass, poloidal magnetic field, electron and inn (proton) densitiee, tcmperatures (elecliun, lon, and neutral gas), $z_{\text {eff }}$, transport and diffusion coefficients, electron and ion temperatures 
at the boundary, [Z], and current densities. The basic output is a new set of plasma variable profiles and resistivity.

\subsection{SUMMARY OF FUNCTION OF EACH SUBROUTINE.}

SUBRØUTINE PLASIM

Subroutine PLASIM is the executive subroutine for the plasma module. It has the sole function of initializing plasma temperatures, densities, electromagnetic field profiles, particle diffusion velocity, and $z_{\text {eff }}$ at the beginning of the simulation. At subsequent times, it calls a series of subroutines which solve the set of coupled parclal diffeleulial equations describing the basic plasma evolution. Figure 4 shows the subroutines which are called and the order of call.

Called from: GETSET [ENTRY IPPC(ITZ)] and MAIN [ENTRY PLASMA(ITZ)]

Subroutines called: CDC, CST, LBM, IBTM

Commons required: 'BDYC $\emptyset \mathrm{N}$, CDCLBM, CDCLBX, CURENT, FIELDS, GE $\emptyset$,

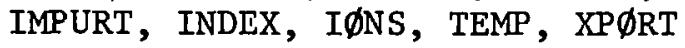

Variables required:

ITZ - time loop index variable

From labeled common blocks (see Appendix II for definitions)

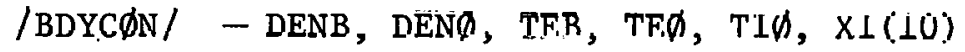

/CURENT/ - ZPC

/GE $\emptyset \mathrm{M} / \quad-\mathrm{HR}, \mathrm{R} 9$

/IMPURT/ - ZEFF

/INDEX/ $-\mathrm{N}$

Variables changed (initialized):

From labeled common blocks (see Appendix II for definitions)

/FIELDS/ - B(101), E(101), ZJ(101), ZJ

/IØNS/ - DEN(LOL)

/TEMP/ - TE(101), TI(101), Tø(101), VEL(101)

/XPфRT/ - DI(101) 
ORNL-DWG 76-2305

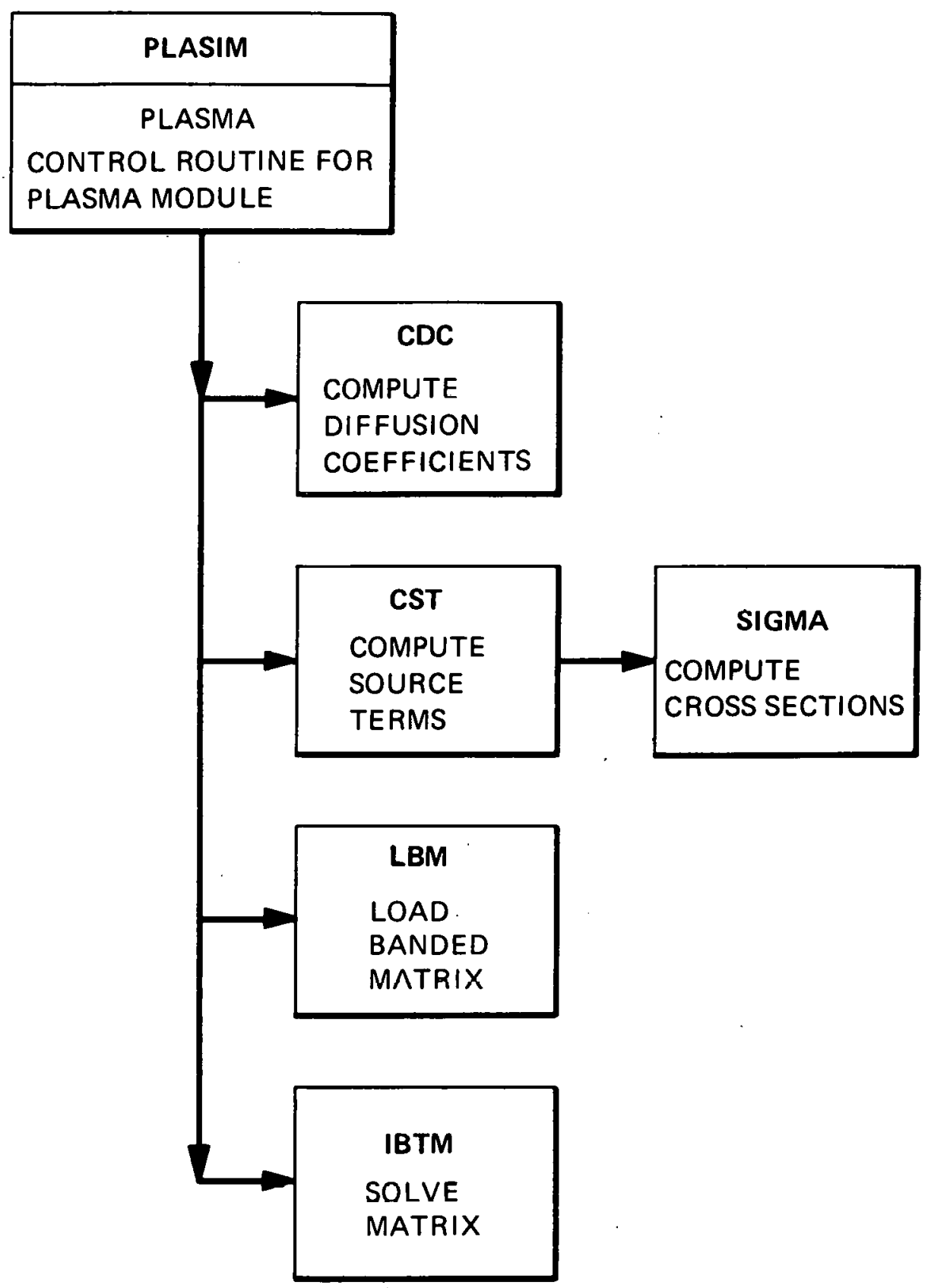

Fig. 4. Diagram of PLASMA module and subroutines called. 


\section{SUBRøUTINE CDC(IT)}

Subroutine CDC computes diffusion coefficients, thermal conductivity profiles, resistivity, and associated derivatives with respect to the plasma variables used in the implicit space-time centered differencing scheme in subroutine LBM. The basic equations used in subroutine CDC and the models associated with them are summarized below.

The electron and ion collision frequencies are given by

$$
\begin{aligned}
& \nu_{e}=\frac{4 \sqrt{2 \pi} e^{4} z^{2} \tilde{N}_{i} \ln \Lambda}{3 \sqrt{\mathrm{m}_{e}} T_{e}{ }^{3 / 2}}=\frac{3.42(10)^{-8} N_{e}\left(\operatorname{cm}^{-3}\right) z_{e f f}}{T_{e}(e V)^{3 / 2}} \frac{\ln \Lambda}{17.25} \\
& \nu_{i}=\frac{4 \sqrt{\pi} \mathrm{e}^{4} \mathrm{Z}^{4} \mathrm{~N}_{i} \ln \Lambda}{3 \sqrt{\mathrm{m}_{i}} \mathrm{~T}_{i}^{3 / 2}}=\frac{8(10)-10 \mathrm{~N}_{i}\left(\mathrm{~cm}^{-3}\right)(1.41) \mathrm{z}_{\mathrm{eff}}}{\sqrt{\mathrm{m}_{\mathrm{p}}(\mathrm{amu})} \mathrm{T}_{i}(\mathrm{eV})^{3 / 2}}=\frac{\ln \Lambda}{17.25}
\end{aligned}
$$

where

$$
\ln \Lambda=17.25-\ln \left\{\frac{\sqrt{10^{-13} \mathrm{~N}_{\mathrm{e}}\left(\mathrm{cm}^{-3}\right)}}{10^{-3} \mathrm{~T}_{\mathrm{e}}(\mathrm{eV})}\right\} \text {. }
$$

The ratios of the collision frequencies to the bounce frequencies are given by

$$
\begin{aligned}
& \nu_{e}^{*} \equiv \frac{\nu_{e}}{\omega_{b, e}}=\nu_{e}\left(m^{-1}\right) R_{o}(c m) B_{T} \text { (gauss) } \sqrt{\frac{R_{o}(\mathrm{~cm})}{r(c m)}} \frac{10^{-7}}{4 \cdot 3 \sqrt{T_{e}(e V)} B_{p}(\text { gauss })} \\
& \sim T_{e} e^{-2 / B_{p}-1} N_{c} \\
& v_{i}^{*}=-\frac{v_{i}}{\omega_{b, i}}-9.9 v_{i}\left(m s e r^{-l}\right) R_{0}\left(\text { rm) } B_{T} \text { (gauss) } \sqrt{\frac{R_{0}(\mathrm{~cm})}{r(c m)} \cdot \sqrt{T_{i}(e V)} B_{P} \text { (gauss) }}\right. \\
& { }^{\sim} \mathrm{T}_{i}^{-2} \mathrm{~B}_{\mathrm{p}}^{-\mathrm{I}_{\mathrm{N}}} \mathrm{p}_{\mathrm{p}}
\end{aligned}
$$

Let $\nu_{e}^{* *}$ be the minimum value of $\hat{D}$ as a function of $\nu_{e}$, and define

$$
f=f\left(\nu_{e}^{*}\right) \equiv 1+\left(\frac{\nu_{e}^{* *}}{\nu_{e}^{*}}\right)^{2} \text {. }
$$

The basic pseudoclassical particle diffusion coefficient is 


$$
D=\nu_{e} \rho_{e} e^{2}\left(\frac{B_{T}}{B_{p}}\right)^{2} \gamma_{3} \text {, in units of } \frac{\mathrm{cm}^{2}}{m s e c} \text {, }
$$

where $\gamma_{3}$ is a coefficient which is given in the input data and $\rho_{e}=\left(3.36 \sqrt{T_{e}}\right) / B_{T}$ is the electron gyro-radius. This diffusion coeffient is modified further to give

$$
\hat{D}=D f\left(\nu_{e}^{*}\right) \sim T_{e}^{-1 / 2} B_{p}^{-2} N_{e} f\left(\nu_{e}^{*}\right)
$$

for the particle diffusion coefficient of the model.

Three derivatives associated with this diffusion coefficient are required in subroutine LBM:

$$
\frac{T_{e}}{\hat{D}} \frac{\partial \hat{D}}{\partial T_{e}}, \quad \frac{B_{p}}{\hat{D}} \frac{\partial \hat{D}}{\partial B_{p}}, \text { and } \frac{N_{e}}{\hat{D}} \frac{\partial \hat{D}}{\partial N_{e}} \text {. }
$$

The first of these derivatives is evaluated to illustrate the form of these derivatives used in subroutine CDC:

$$
\begin{aligned}
& \frac{T_{e}}{\hat{D}} \frac{\partial \hat{D}}{\partial T_{e}}=\frac{T_{e}}{\hat{D}}\left\{\frac{\partial \hat{D}}{\partial D} \frac{\partial D}{\partial T_{e}}+\frac{\partial \hat{D}}{\partial f} \frac{\partial f}{\partial \nu_{e}^{*}} \frac{\partial \nu_{e}^{*}}{\partial T_{e}}\right\} \\
& \frac{\partial \hat{D}}{\partial D}=f \quad \frac{\partial D}{\partial T_{e}}=\frac{1}{2} \frac{D}{T_{e}} \\
& \frac{\partial \hat{D}}{\partial f}=D \quad \frac{\partial f}{\partial \nu_{e}^{*}}=-\frac{2}{\nu_{e}^{*}}\left(\frac{\nu_{e}^{* *}}{\nu_{e}^{*}}\right)^{2} \quad \frac{\partial \nu_{e}^{*}}{\partial T_{e}}=-\frac{\partial \nu_{e}^{*}}{T_{e}}
\end{aligned}
$$

so that

$$
\begin{aligned}
\frac{T_{e}}{\hat{D}} \frac{\partial \hat{D}}{\partial T_{e}} & =\frac{T_{e}}{\hat{D}}\left\{-\frac{D}{\partial T_{e}}+4 \frac{D}{T_{e}}\left(\frac{\nu_{e}^{* *}}{v_{e}^{*}}\right)^{2}\right\} \\
& =-\frac{1 / 2}{2}+\frac{4\left(\nu_{e}^{* *}\right)^{2}}{\left(\nu_{e}^{*}\right)^{2}+\left(\nu_{e}^{*}\right)^{2}}
\end{aligned}
$$


In like manner,

$$
\frac{B_{p}}{\hat{D}} \frac{\partial \hat{D}}{\partial B_{p}}=-2+2 \cdot \frac{\left(\nu_{e}^{* *}\right)^{2}}{\left(\nu_{e}^{* *}\right)^{2}+\left(\nu_{e}^{*}\right)^{2}}
$$

and

$$
\frac{\mathrm{N}_{\mathrm{e}}}{\hat{\mathrm{D}}} \frac{\partial \hat{\mathrm{D}}}{\partial \mathrm{N}_{\mathrm{e}}}=1-2 \frac{\left(\nu_{\mathrm{e}}^{* *}\right)^{2}}{\left(\nu_{e^{* *}}\right)^{2}+\left(\nu_{e^{*}}\right)^{2}} \text {. }
$$

The basic pseudo-classical electron thermal diffusion coefficient is

$$
x_{e}=\nu_{e} \rho_{e}^{2}\left(\frac{B_{T}}{B_{p}}\right)^{2} \gamma_{l} \text {, in units of }\left[\frac{\mathrm{cm}^{2}}{m s e c}\right] \text {, }
$$

where $\gamma_{1}$ is a coefficient which is given in the input data. The coefficient is modified further according to

$$
\hat{x}_{e}=x_{e} f\left(\nu_{e}^{*}\right) \sim T_{e}^{-1 / 2} B_{p}^{-2} N_{e} f\left(\nu_{e}^{*}\right) \text {. }
$$

Three derivatives associated with this diffusion coefficient are also required for subroutine LBM and are given by

$$
\begin{aligned}
& \frac{T_{e}}{\hat{x}_{e}} \frac{\partial \hat{x}_{e}}{\partial T_{e}}=-\frac{1}{2}+4 \frac{\left(\nu_{e}^{* *}\right)^{2}}{\left(\nu_{e}^{* *}\right)^{2}+\left(\nu_{e}^{*}\right)^{2}} \\
& \frac{{ }_{p}}{\hat{x}_{e}} \frac{\partial \hat{x}_{e}}{\partial B_{p}}=-2+2 \frac{\left(\nu_{e}^{* *}\right)^{2}}{\left(\nu_{e}^{* *}\right)^{2}+\left(\nu_{e}^{*}\right)^{2}} \\
& \frac{N_{e}}{\hat{x}_{e}} \frac{\partial \hat{x}_{e}}{\partial N_{e}}=1-2 \frac{\left(\nu_{e}^{* *}\right)^{2}}{\left(\nu_{e}^{* *}\right)^{2}+\left(\nu_{e}^{*}\right)^{2}}
\end{aligned}
$$


The basic neoclassical ion (proton) thermal diffusion coefficient is

$$
x_{i}=1.41 \nu_{i} \rho_{i}^{2} \sqrt{R_{0}}\left(\frac{B_{T}}{B_{p}}\right)^{2} \gamma_{2} \text {, in units of } \frac{\mathrm{cm}^{2}}{\mathrm{msec}} \text {, }
$$

where $\gamma_{2}$ is a coefficient given in the input data and $\rho_{i}=\frac{\sqrt{2.09(10)^{4} \mathrm{~m}_{\mathrm{p}} \mathrm{T}_{i}}}{\mathrm{~B}_{\mathrm{T}}}$ is the proton gyro-radius. This diffusion coefficient is also modified further to give

$$
\hat{x}_{i}=x_{i} \frac{0.47\left[1+0.43 \nu_{i}^{*}\left(\frac{r}{R_{0}}\right)^{3 / 2}\right]}{1+1.03 \sqrt{\nu_{i}^{*}}+0.18 \nu_{i}^{*}}=x_{i} g\left(\nu_{i}^{*}\right) \sim \mathrm{T}_{i}{ }^{-1 / 2} \mathrm{~B}_{\mathrm{p}}^{-2} \mathrm{~N}_{i} g\left(\nu_{i}^{*}\right) \text {. }
$$

The three derivatives associated with this diffusion coefficient are given by

$$
\frac{\mathrm{T}_{i}}{\hat{x}_{i}} \frac{\partial \hat{x}_{i}}{\partial \mathrm{T}_{i}}=\frac{\mathrm{T}_{i}}{\hat{x}_{i}}\left\{\frac{\partial \hat{x}_{i}}{\partial x_{i}} \frac{\partial x_{i}}{\partial \mathrm{T}_{i}}+\frac{\partial \hat{x}_{i}}{\partial g} \frac{\partial g}{\partial \nu_{i}^{*}} \frac{\partial \nu_{i}^{*}}{\partial \mathrm{T}_{i}}\right\}
$$

where

$$
\begin{aligned}
& \frac{\partial \hat{x}_{i}}{\partial x_{i}} \frac{\partial x_{i}}{\partial T_{i}}=g\left(\nu_{i}{ }^{*}\right)\left[-\frac{1}{2} \frac{x_{i}}{T_{i}}\right]=-\frac{1}{2} \frac{\hat{x}_{i}}{T_{i}} \\
& \frac{\partial g}{\partial \nu_{i}^{*}}=\frac{0.47\left\{0.43\left(\frac{r}{R_{0}}\right)^{3 / 2}\left(1+0.52 \sqrt{v_{i}^{*}}\right)-\left(\frac{0.51}{\sqrt{v_{i}^{*}}+0.18}\right)\right\}}{\left[1+1.03 \sqrt{v_{i}^{*}}+0.18 v_{i}^{*}\right]^{2}} \\
& \frac{\partial \hat{x}_{i}}{\partial g}=x_{i} \text { and } \frac{\partial \nu_{i}^{*}}{\partial T_{i}}=-\frac{2 \nu_{i}^{*}}{T_{i}} \text {; }
\end{aligned}
$$

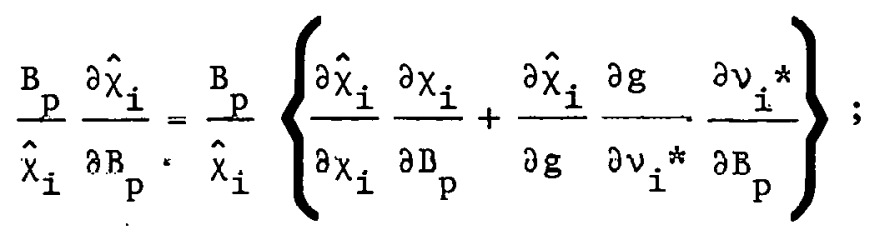


where

$$
\frac{\partial \hat{x}_{i}}{\partial x_{i}} \frac{\partial x_{i}}{\partial B_{p}}=g\left[-\frac{2 x_{i}}{B_{p}}\right]=-\frac{2 \hat{x}_{i}}{B_{p}} \text { and } \frac{\partial \nu_{i}^{*}}{\partial B_{p}}=-\frac{\nu_{i}^{*}}{B_{p}} \text {; }
$$

and

$$
\frac{N_{i}}{\hat{x}_{i}} \frac{\partial \hat{x}_{i}}{\partial N_{i}}=\frac{N_{i}}{\hat{x}_{i}}\left\{\frac{\partial \hat{x}_{i}}{\partial x_{i}} \frac{\partial x_{i}}{\partial N_{i}}+\frac{\partial \hat{x}_{i}}{\partial g} \frac{\partial g}{\partial v_{i}^{*}} \frac{\partial \nu_{i}^{*}}{\partial N_{i}}\right\}
$$

where

$$
\frac{\partial \hat{x}_{i}}{\partial x_{i}} \frac{\partial x_{i}}{\partial N_{i}}=\frac{\hat{x}_{i}}{N_{i}} \text { and } \frac{\partial \nu_{i}^{*}}{\partial N_{i}}=\frac{\nu_{i}^{*}}{N_{i}}
$$

The three diffusion coefficients and their associated derivatives are evaluated separately for their values at the origin:

$$
\begin{aligned}
& \hat{D}(r=0)=\hat{D}(r=\Delta r) \quad \frac{T_{e}}{\hat{D}} \frac{\partial \hat{D}}{\partial T_{e}}=1 \frac{B_{p}}{\hat{D}} \frac{\partial \hat{D}}{\partial B_{p}}=0 \frac{N_{e}}{\hat{D}} \frac{\partial \hat{D}}{\partial N_{e}}=0 \\
& \hat{x}_{e}(r=0)=\hat{x}_{e}(r=\Delta r) \frac{T_{e}}{\hat{x}_{e}} \frac{\partial \hat{x}_{e}}{\partial T_{e}}=1 \frac{B_{p}}{\hat{x}_{e}} \frac{\partial \hat{x}_{e}}{\partial B_{p}}=0 \frac{N_{e}}{\hat{x}_{e}^{\prime}} \frac{\partial \hat{x}_{e}}{\partial N_{e}}=0 \\
& \hat{\chi}_{i}(r=0)=\left[1.6+\frac{(3.7)(980) j \sqrt{T_{i}}}{1.6 B_{T} \nu_{i}}\right]\left(\frac{1.6 B_{T}}{R_{o} j}\right)^{2} \nu_{i}\left[\frac{2.09(10)^{4} M_{p} T_{i}}{B_{T}^{2}}\right] \\
& \left.\frac{T_{i}}{\hat{x}_{i}} \frac{\partial \hat{x}_{i}}{\partial T_{i}}\right\rangle_{r=0}=-\frac{1}{2}+\frac{(4.65)(980) j \sqrt{T_{i}}}{1.6 B_{T} \nu_{i}}
\end{aligned}
$$




$$
\begin{aligned}
& \left.\frac{{ }_{\mathrm{p}}}{\hat{\mathrm{x}}_{i}} \frac{\partial \hat{\mathrm{x}}_{i}}{\partial \mathrm{B}_{\mathrm{p}}}\right\}_{\mathrm{r}=0}=-2+\frac{(2.35)(980) j \sqrt{\mathrm{T}_{i}}}{1.6 \mathrm{~B}_{\mathrm{T}}{ }{ }_{i}} \\
& \left.\frac{\mathrm{N}_{i}}{\hat{\mathrm{x}}_{i}} \frac{\partial \hat{\mathrm{x}}_{i}}{\partial \mathrm{N}_{i}}\right\}_{r=0}=1-\frac{(2.35)(980) j \sqrt{\mathrm{T}_{i}}}{1.6 \mathrm{~B}_{\mathrm{T}}{ }{ }_{i}}
\end{aligned}
$$

\section{Called from:}

Commons required:

Variables required

IT

\section{PLASIM [ENTRY PLASMA]}

CDCLBM, CDCLBX, GE $\emptyset$, FIELDS, IMPURT, INDEX, IøNS, TEMP, THRMDF, XPØRT, XTRA

(see Table 6 and Appendix II)

- time loop index variable

Arrays changed:

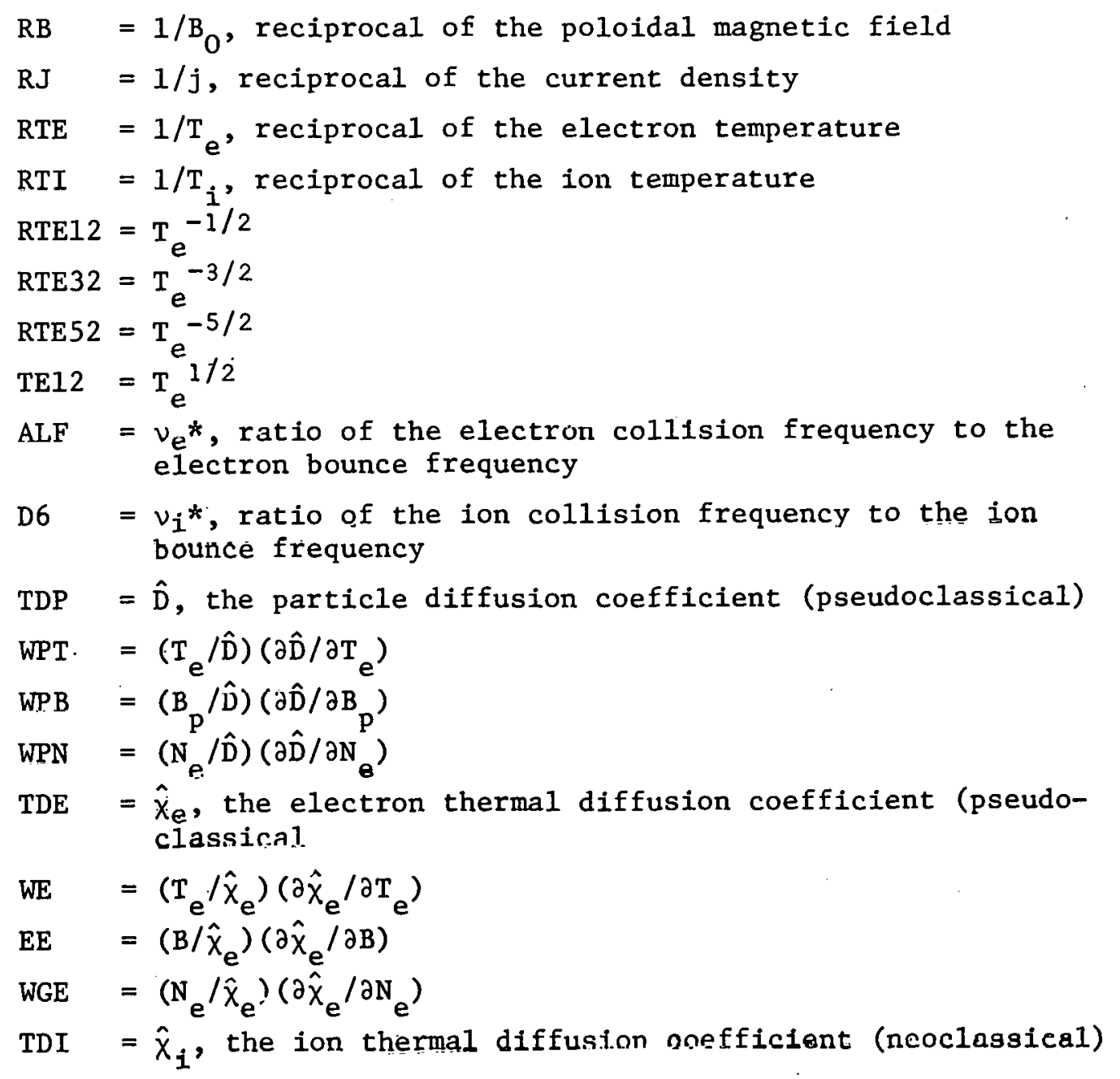




$$
\begin{aligned}
\text { WI } & =\left(\mathrm{T}_{i} / \hat{x}_{i}\right)\left(\partial \hat{x}_{i} / \partial \mathrm{T}_{i}\right) \\
\mathrm{EI} & =\left(\mathrm{B} / \hat{\mathrm{x}}_{i}\right)\left(\partial \hat{\mathrm{x}}_{i} / \partial \mathrm{B}\right) \\
\text { WGI } & =\left(\mathrm{N}_{i} / \hat{x}_{i}\right)\left(\partial \hat{x}_{i} / \partial \mathrm{N}_{i}\right) \\
\text { ETA } & =n, \text { the plasma resistivity }
\end{aligned}
$$

Significant internal variables:

$\operatorname{CZE}=\frac{4 \sqrt{2 \pi} \mathrm{e}^{4} \mathrm{~N}_{\mathrm{p}}}{3 \sqrt{\mathrm{m}_{\mathrm{e}}}}=3.42(10)^{-8} \mathrm{~N}_{\mathrm{p}}, \mathrm{N}_{\mathrm{p}} \equiv$ proton density

C.ZI $=\frac{4 \sqrt{\pi} e^{4} \mathrm{~N}_{\mathrm{P}}}{3 \sqrt{\mathrm{m}_{\mathrm{p}}}}=8(10)^{-10} \frac{\mathrm{N}_{\mathrm{p}}}{\sqrt{\mathrm{m}_{\mathrm{p}}}}$

$\mathrm{ZL} \quad=\frac{\ln \Lambda}{17.25}=\frac{1}{17.25}\left\{1-\ln \left[\frac{\sqrt{10^{-13} \mathrm{~N}_{\mathrm{e}}}}{10^{-3} \mathrm{~T}_{\mathrm{e}}}\right]\right\}$, the Coulomb

logarithm factor

ZNUE $=\nu_{e} \equiv \frac{1}{\tau_{e}}=3.42(10)^{-8} \mathrm{~N}_{\mathrm{p}} \mathrm{T}_{\mathrm{e}}^{-3 / 2} \mathrm{z}_{\text {eff }} \frac{\ln \Lambda}{17.25}$, the electron collision frequency, $\mathrm{msec}^{-1}$

7.NITT $=v_{1} \equiv \frac{1}{\tau_{1}}=8(10)^{-10} \frac{\mathrm{N}_{\mathrm{p}}}{{\sqrt{\mathrm{m}_{\mathrm{p}}}}_{\mathrm{p}}} \mathrm{T}_{\mathrm{i}}{ }^{-3 / 2} \sqrt{2} \mathrm{z}_{\text {eff }} \frac{\ln \Lambda}{17.25}$, the ion collision frequency, $\mathrm{msec}^{-1}$

QQ $=\frac{\mathrm{rB}_{\mathrm{T}}}{\mathrm{R}_{\mathrm{O}} \mathrm{B}_{\mathrm{P}}}$, the safety factor

RKI $=\frac{0.47\left[1+0.43 v_{i}^{*}\left(\frac{r}{R_{n}}\right)^{3 / 2}\right]}{1+1.03 \sqrt{v_{i}^{*}+0.18 v_{i}^{*}}}$, the lun thermal diffuoion modification factor

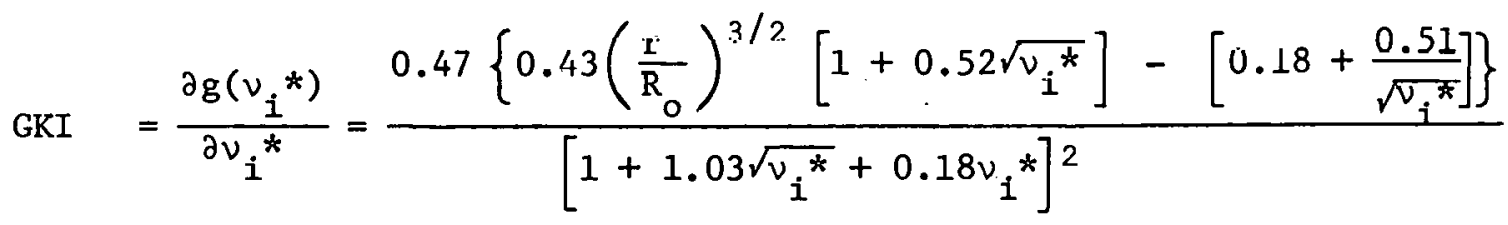




$$
\begin{aligned}
& \text { FACT }=f\left(\nu_{e}^{*}\right)=1+\left(\frac{v^{* *}}{v_{e}^{*}}\right)^{2} \\
& \operatorname{TRAP}=\frac{f\left(\nu_{\left.e^{*}\right)-1}\right.}{f\left(\nu_{e^{*}}\right)}=\frac{\left(\nu_{e^{* *}}\right)^{2}}{\left(\nu_{e^{* *}}\right)^{2}+\left(\nu_{e^{*}}\right)^{2}} \\
& R H \emptyset E 2=\rho_{e}^{2}=\left(\frac{3.36}{B_{T}}\right)^{2} T_{e} \text {, the square of the electron gyroradius } \\
& \text { RHøI2 }=\rho_{i}^{2}=\frac{2.09(10)^{4} m_{p^{T}}}{B_{T}^{2}} \text {, the square of the ion gyroradius }
\end{aligned}
$$


Table 6. List of labeled cormor.s and elements within them usec jy subroutine CDC

\begin{tabular}{|c|c|c|c|c|}
\hline \multirow{2}{*}{$\mathrm{C} \emptyset \mathrm{MM} \phi \mathbb{N} \quad \mathrm{BL} \emptyset \mathrm{CK}$} & \multicolumn{2}{|c|}{ Inpu: } & \multicolumn{2}{|c|}{ Output } \\
\hline & Variables & Arrays & Variables & Arrays \\
\hline /CDCLBM/ & & & & $\begin{array}{l}\text { EE, EI, RB, RJ, } \\
\text { RTE, RTI, RTE12, } \\
\text { RTE32, RTE52, TDP, } \\
\text { WE, WI }\end{array}$ \\
\hline /CDCLBX/ & & & & $\begin{array}{l}\text { WPT, WPB, WPN, ETA, } \\
\text { WGE, WGI }\end{array}$ \\
\hline /GE $\emptyset \mathrm{M} /$ & $H R, \quad R \emptyset$ & & & \\
\hline /FIELDS/ & BT & $\mathrm{E}, \mathrm{ZJ}$ & & \\
\hline / INPURT / & & INENP & & \\
\hline / INDEX/ & $\mathrm{N}$ & & & \\
\hline /IøNS/ & $\mathrm{AMC}^{-}$ & IDET & & \\
\hline /TEMP/ & & $\mathrm{TE}: \mathrm{TI}, \mathrm{T} \emptyset$ & & \\
\hline /THRMDF/ & & & . & TDE, TDI \\
\hline /XPøRT/ & & $\mathrm{D} 1, \mathrm{D} 11$ & & D6 \\
\hline /XTRA/ & & & & ALF, TE12 \\
\hline
\end{tabular}




\section{SUBR $\emptyset$ UT INE CST}

Subroutine CST computes the source terms: i.e., the particles and power delivered to the electrons and ions. Contributions to the electron power are a gain from the injected neutral beam heat input, losses due to heating newly ionized neutral hydrogen by electron impact, and radiation losses due to electron impact with impurities. Contributions to the proton power are a gain from the injected neutral beam heat input, losses due to heating newly' created protons, and charge exchange. The rate $\mathrm{R}_{\text {ie }}$ for ionization of neutral hydrogen by electron impact is computed as

$$
\mathrm{R}_{\text {ie }}=\mathrm{N}_{\mathrm{p}} \mathrm{N}_{\mathrm{o}}\left\langle\sigma v{ }_{\text {ionization }}, \sec ^{-1}\right.
$$

where $\langle\sigma \nu\rangle_{i o n}$ is the rate coefficient for neutral hydrogen ionization by electron impact.

\section{Called from: PLASIM [ENTRY PLASMA]}

Subroutines called: SIGMA

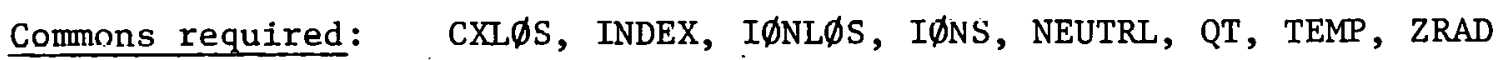

Variables required:

From labeled common blocks (see Appendix II for definitions).

$$
\begin{aligned}
& \text { /INDEX/ }-\mathrm{N} \\
& \text { /IONS/ }-\mathrm{DEN}(101) \\
& \text { /NEUTRL/ - ZN1(101) } \\
& \text { /TEMP/ - TE(101), TI(101), T } \emptyset(101) \\
& \text { /ZRAT/ - QRD(101) }
\end{aligned}
$$

Arrays changed:

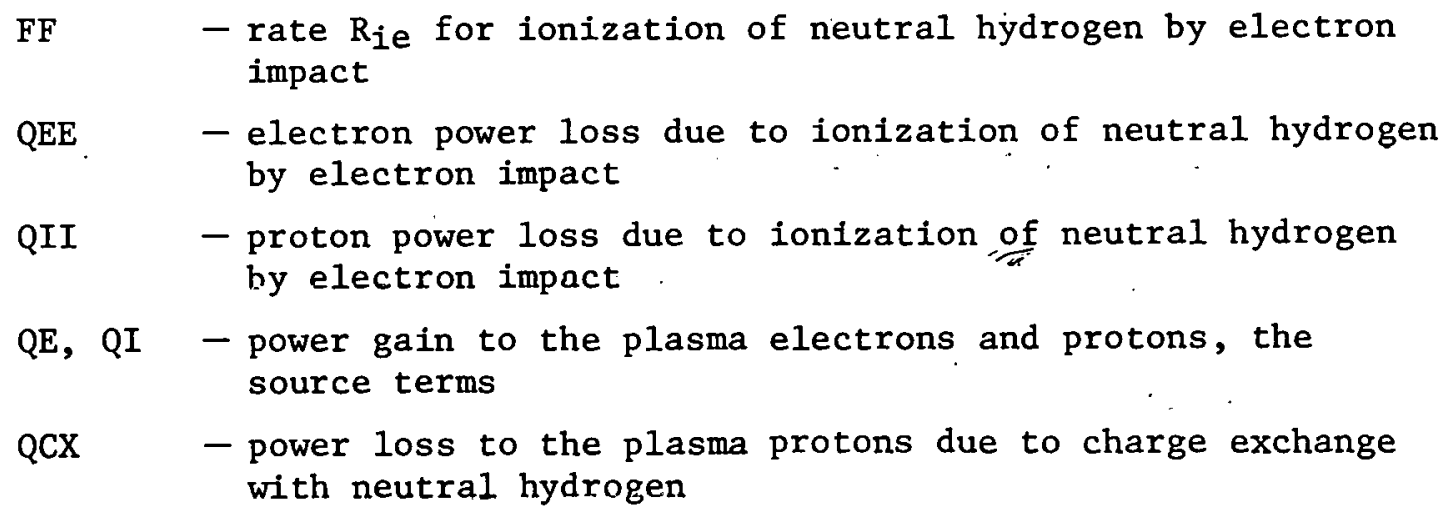


SUBRØUTINE LBM(IT)

Subroutine LBM calculates the elements of the banded matrix which is used in subroutine IBTM to give the solution to the plasma variable profiles at each new time step. The method used here was originally devised by Widner and Dory. ${ }^{2}$ The banded matrix is loaded according to a scheme which assigns a unique location MIJK according to the spatial grid number (I) used for the differencing, the model equation number ( $J$ ), and the variable number $(K)$. This scheme is summarized in Table 7.

The differencing scheme is straightforward conceptually, but can bccome tedinus and time-consuming to work out in practice. The method is summarized so that if a user wants to change the model, he will have a reference for proceeding to derive the new matrix elements required. The general form of the equations is

$$
\frac{\partial u}{\partial t}=\frac{1}{r} \frac{\partial}{\partial r}\left[r g \frac{\partial h}{\partial r}\right]+A \equiv r h s
$$

Let the subscripts $I-1, I$, and $I+1$ be used to denote spatial grid points for $r-\Delta r, r$, and $r+\Delta r$. Let superscripts $N, N+\frac{1}{2}$, and $N+1$ denote time grid points for $t, t+\frac{\Delta t}{2}$, and $t+\Delta t$. The rules for evaluating the matrix ele- 0 ments are as follows:

1. Time derivatives: $\frac{\partial u}{\partial t}=\frac{1}{\Delta t}\left[u^{N+1}-u^{N}\right]$

2. First order spatial derivatives: $\frac{\partial h}{\partial r}=\frac{1}{2 \Delta r}\left[h_{I+1}^{N}-h_{I-1}^{N}\right]$

3. Second order opatial derivatives: $\frac{\partial^{2} h}{\partial r^{2}}=\left(\frac{1}{\Delta r}\right)^{2}\left[h_{I+1}^{N}-2 h_{I}^{N}+h_{T-1}^{N}\right]$

4. Each plasma variable $\phi$ on the tiglit-hand nido of the equation is evaluated at the midpoint of the interval $(t, t+\Delta t)$ according to the prescription:

$\left.[\phi]_{I}^{N+1 / 2}=1 / 2\left\{[\phi]^{N+1}+L_{r}\right]^{N}\right\}$ 
Table 7. Matrix element assignment scheme for banded matrix MIJK

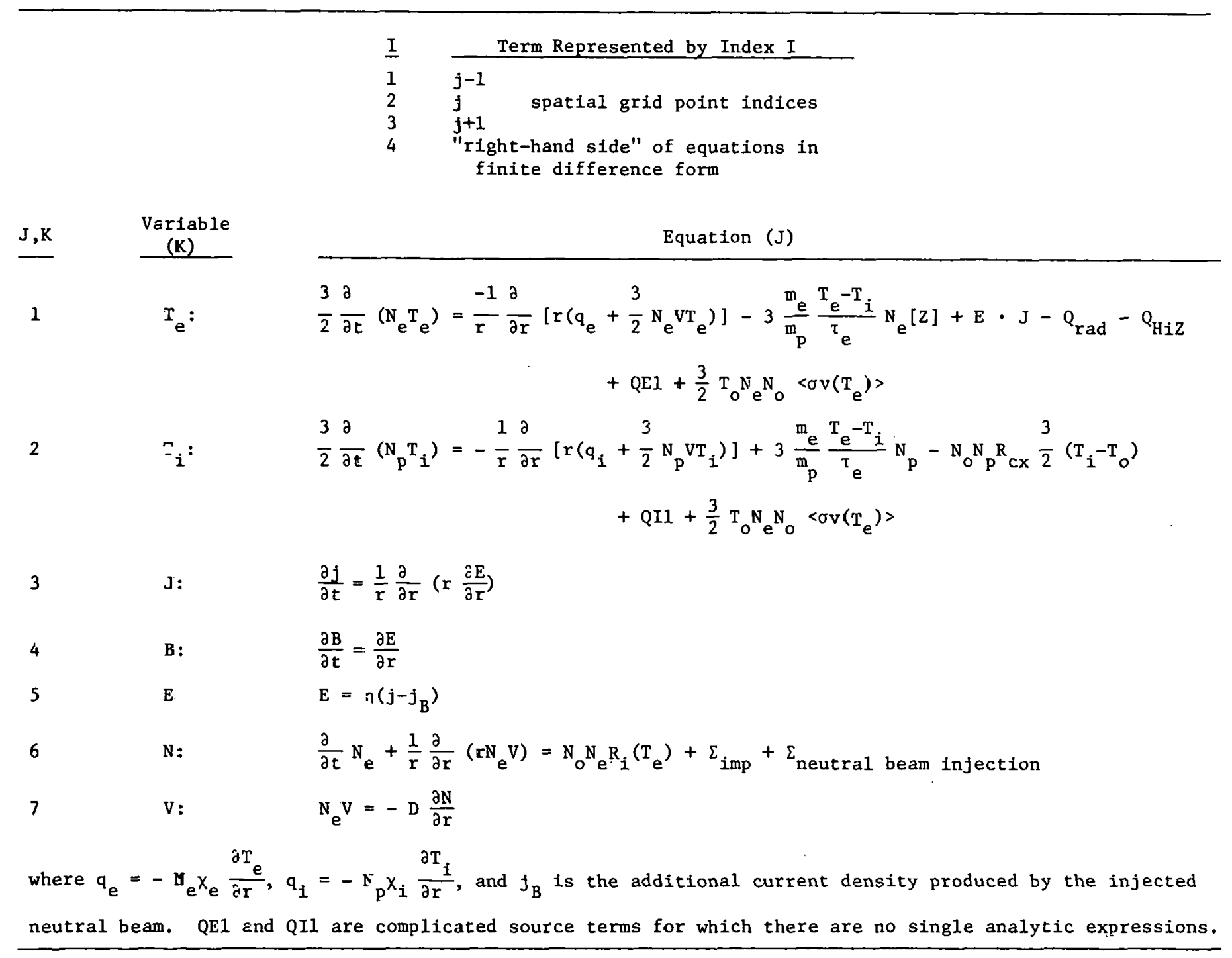


where

$$
\begin{aligned}
& {[\phi]^{N+1}=[\phi]^{N}+\Delta t \frac{\partial[\phi]}{\partial t}+\left[o(\Delta t)^{2}\right]} \\
& \text { For the purposes of the computations, one is actually evaluating } \\
& {\left[\frac{\partial u}{\partial t}\right]_{I}=R\left\{\left[\phi_{a}\right]_{I}^{N+1 / 2},\left[\phi_{b}\right]_{I}^{N+1 / 2}, \cdots\left[\phi_{n}\right]_{I}^{N+1 / 2}\right\}}
\end{aligned}
$$

Once all the equations have been created lil this faghion, they will be i.11 the form

$$
\begin{aligned}
\left\{\alpha_{1}\right\} \mathrm{T}_{\mathrm{e}_{I-1}^{N+1}}^{\mathrm{N}+1} & +\left\{u_{2}\right\} \mathrm{T}_{\mathrm{e}_{I}+1}^{\mathrm{N}+1}+\left\{\alpha_{3}\right\} \mathrm{T}_{\mathrm{e}_{I+1}^{N+1}}^{\mathrm{N}}+\left\{\beta_{1}\right\} \mathrm{T}_{\mathrm{I}_{I-1}}^{\mathrm{N}+1}+\left\{\beta_{2}\right\} \mathrm{T}_{{ }_{I}}^{\mathrm{N}+1} \\
& +\ldots=\text { RHS }
\end{aligned}
$$

where the $\alpha_{i}, \beta_{i}$, etc. are the matrix elements. Using the $T_{e}$ equation as an example, the matrix elements MIJK would be

$$
\begin{aligned}
& \mathrm{M} 111=\alpha_{1} \quad \mathrm{M} 112=B_{1} \quad \ldots \quad \text { M411 }=\text { RHS } \\
& M 211=N_{2} \quad M 212=B_{2} \quad \ldots \quad \text { etc. } \\
& \text { M311 }=\alpha_{3} \quad \ldots \quad \quad \ldots
\end{aligned}
$$

Time derivarives Luvulving the diffuoion and transport rnefflclents should be handled in a general way using the chain rule and functional derivatives according to

$$
\frac{\partial D}{\partial L}=\frac{\partial D}{\partial T} \frac{\partial ' T}{e} \quad, \ldots
$$

since subruuline CDC compucee $\frac{\mathrm{e}}{\mathrm{I}} \frac{\partial \mathrm{d}}{\partial \mathrm{T}}, \ldots$ (ace the descriptinn of subroutine $\mathrm{CDC}$, Arrays changed). The correspondence between the matrix elements in LBM and the terms in the partial differential equations is shown in Table 8. 
Table 8. Correspondence between terms in plasma equations and matrix elements [IJK] in LBM.

\section{$\mathrm{T}_{\mathrm{e}}$ Equation}

$$
\begin{aligned}
& \frac{3}{2} \cdot \frac{\partial}{\partial t}\left(N_{e} T_{e}\right)=-\frac{1}{r} \frac{\partial}{\partial r}\left[r\left(q_{e}+\frac{3}{2} N_{e} V T_{e}\right)\right]-3 \frac{m_{e}}{m_{p}} \frac{T_{e}-T_{i}}{\tau_{e}}[Z] N_{e}+E \cdot j+\frac{3}{2} T_{o} N_{e} N_{o}\left\langle\sigma v\left(T_{e}\right)\right\rangle+Q_{r e m}
\end{aligned}
$$

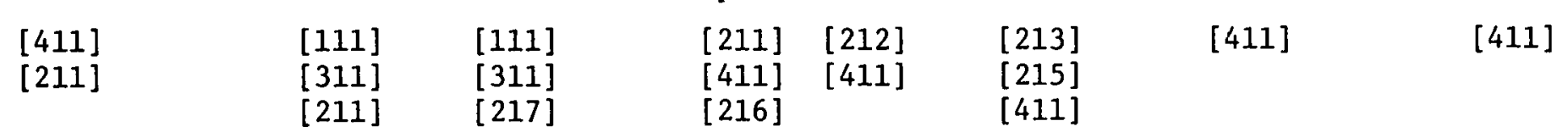

$$
\begin{aligned}
& \text { [114] [117] } \\
& \begin{array}{ll}
{[214]} & {[317]} \\
{[314]} & {[116]}
\end{array} \\
& \text { [116] [216] } \\
& \text { [216] [316] } \\
& \text { [316] [411] } \\
& \text { [411] } \\
& \text { where } Q_{\text {rem }}=Q E 1-Q_{\text {rad }}-Q_{H i Z}
\end{aligned}
$$

$T_{i}$ Equation

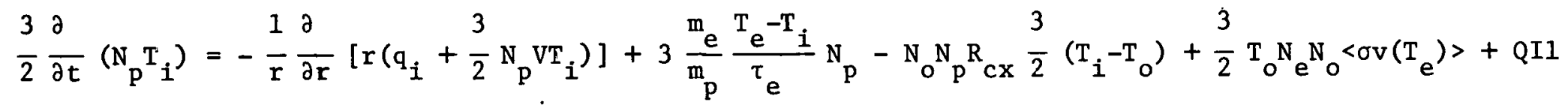

$$
\begin{aligned}
& \text { [421] } \\
& \text { [222] } \\
& \begin{array}{ll}
{[122]} & {[122]} \\
{[322]} & {[322]} \\
{[124]} & {[227]} \\
{[224]} & {[421]} \\
{[324]} & {[127]} \\
{[421]} & {[327]}
\end{array} \\
& \begin{array}{ll}
\text { [221] } & \text { [222] } \\
{[421]} & {[421]}
\end{array} \\
& \text { [222] } \\
& \text { [222] } \\
& \text { [421] }
\end{aligned}
$$


Tatle 8 (continued)

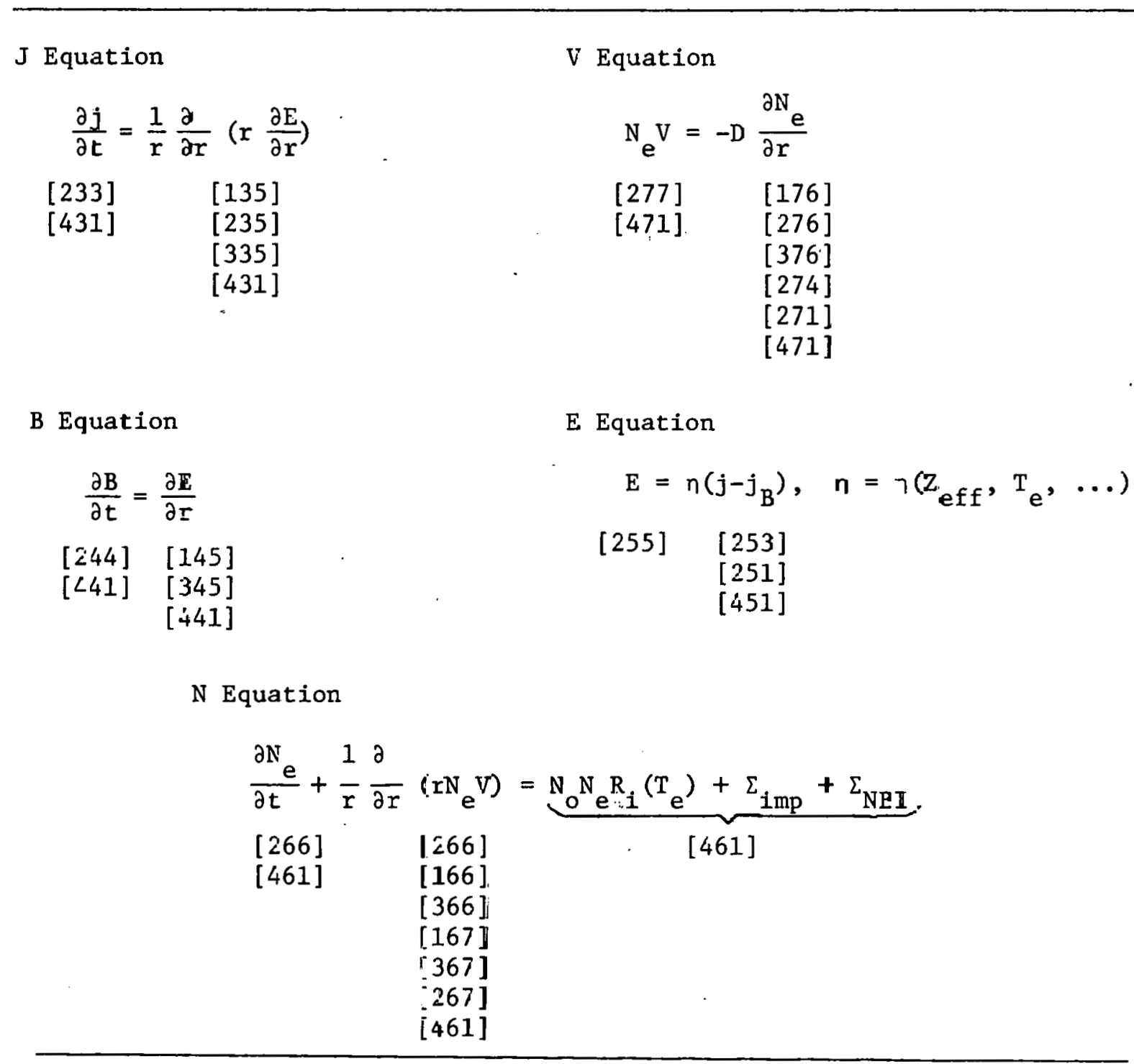


The matrix elements MIJK occur for each of the grid points in the spatial net, so the general matrix element is $A(M X+M I J K)$, with $\mathrm{MX}=0 * \mathrm{MK}, 1 * \mathrm{MK}, 2 * \mathrm{MK}, \ldots, \mathrm{N \& MK}$ where $\mathrm{N}$ is the number of radial grid points and $M K=(3 K+1) K$, with $K$ the number of equations (seven). The spatial net is divided into three intervals corresponding to $\mathrm{MX}=0 * \mathrm{MK}$, $1 * \mathrm{MK}$ through $(\mathrm{N}-1) * \mathrm{MK}$, and $\mathrm{N}^{*} \mathrm{MK}$. The matrix elements for $\mathrm{MX}=1 * \mathrm{MK}$ through $(\mathrm{N}-1) \times \mathrm{MK}$ all have the same general form while those for $\mathrm{MX}=0 * \mathrm{MK}$ and $\mathrm{N}^{*} \mathrm{MK}$ are different since the boundary conditions must be incorporated at these two end points. At the plasma center ( $M X=0 * M K)$, the boundary conditions are

$$
\begin{aligned}
& \frac{\mathrm{dT}_{\mathrm{e}}}{\mathrm{dr}}=\frac{\mathrm{dT}_{i}}{\mathrm{dr}}=\frac{\mathrm{dN}}{\mathrm{dr}}=0 ; \\
& B(r=0)=0 ; \\
& j=\nabla \times B ; \\
& E=n j ;
\end{aligned}
$$

and at the plasma edge $\left(M X=N^{*} M K\right)$, the boundary conditions are

$$
\begin{aligned}
& T_{e, i}(a)=T_{e, i} ; \quad N(a)=N_{e}^{a} ; B(a)=.2 I / a ; \\
& j=\nabla \times B \text { (extrapolating from the values of } B \text { and } \frac{d B}{d r} \text { at } r=a-\Delta r \text { ); } \\
& E=n j .
\end{aligned}
$$

A few matrix elements are evaluated in Appendix III to illustrate the method for a simple case and a complex one.

\section{Called from: PLASIM [ENTRY PLASMA]}

Subroutines called: $\mathrm{PC}$

Commons required: $\quad B D Y C \emptyset N, B E A M C, C D C L B M, C D C L B X, G E \emptyset M$, FIELDS, IMPURT, INDEX I $\emptyset L \emptyset S, I \emptyset N S$, LBMIBT, NEUTRL, QT, TEMP, THRMDF, TIME, XPøRT

Variables required: (See Table 9 and Appendix II)

IT - time loop index variable

Array changed:

A - matrix of coefficients of the system of differenced equations 
Table 9. List cf labeled commons and əlements within them used ty subroutine LBM

\begin{tabular}{|c|c|c|c|c|}
\hline \multirow{2}{*}{$\mathrm{C} \emptyset \mathrm{MM} \emptyset \mathrm{N} \quad \mathrm{BL} \emptyset \mathrm{C} \mathrm{K}$} & \multicolumn{2}{|c|}{ Input } & \multicolumn{2}{|c|}{ Output } \\
\hline & Variebles & Arrays & Variables & Arrays \\
\hline$/ \mathrm{BDYC} \emptyset \mathrm{N} /$ & -EQ, TIф & & & \\
\hline /BEAMC/ & & DJT & & \\
\hline /CDCLBM/ & & $\begin{array}{l}\text { EE, EI, RB, RTE, } \\
\text { RTI, RTE12, } \\
\text { RTE3.2, RTE52, } \\
\text { TDP, WE, WI }\end{array}$ & . & \\
\hline /CDCLBX/ & & WPT, WPB, WPN, ETA & & $\cdot$ \\
\hline /FIELDS/ & & $\mathrm{B}, \mathrm{E}, \mathrm{ZJ}$ & & \\
\hline /GE $\emptyset \mathrm{M} /$ & $A M, \mathbb{H R}$ & & & \\
\hline /IMPURT/ & & DENP, ZBR & & \\
\hline /INDEX/ & $\mathrm{N}, \mathrm{NMI}$ & & & \\
\hline /IøNL ØS / & & $\mathrm{FF}$ & & \\
\hline /IøNS/ & AMU & DEN & & \\
\hline /LBMIBT/ & . & & & $A^{\circ}$ \\
\hline /NEUTRL/ & & ZN1 & & \\
\hline /QT/ & & $\overline{Z E}, Q \mathrm{I}$ & & \\
\hline /TEMP / & & $\mathrm{TE}, \mathrm{TI}, \mathrm{T} \emptyset, \mathrm{VEL}$ & & \\
\hline /THRMDF/ & & TDE, TDI & & \\
\hline /TIME/ & HT, TIM & & & \\
\hline$/ \mathrm{XP} \emptyset \mathrm{RT} /$ & & $\overline{Z L}$ & & \\
\hline
\end{tabular}


SUBRøUTINE IBTM(IT)

Subroutine IBTM approximates the solution to the system of linear equations

$\mathrm{FX}=\mathrm{B}$

where $\mathrm{F}$ is a block tridiagonal matrix of order $\mathrm{N}$ and each block element of $\mathrm{F}$ is $\mathrm{a} \mathrm{K}$ by $\mathrm{K}$ submatrix. The approximation is determined by Gaussian elimination without any pivoting. Let $F(I, J)$ denote the $(I, J)$ block element of the matrix $F$ and let $B(I)$ denote the associated $K$ element subvector of the vector $B$. The $3 K+1$ by $N * K$ array $A$ in common LBMIBT contains on entry into IBTM the nontrivial information to describe the coefficient matrix $F$ and the right-hand side $B$. The transpose of the array $A$ is displayed since it portrays the information more naturally.

$$
\operatorname{transpose}(\mathrm{A})=\left(\begin{array}{cccc}
0 & \mathrm{~F}(1,1) & \mathrm{F}(1,2) & \mathrm{B}(1) \\
\mathrm{F}(2,1) & \mathrm{F}(2,2) & \mathrm{F}(2,3) & \mathrm{B}(2) \\
\dot{*} & \dot{\cdot} & \dot{\cdot} & \cdot \\
\dot{\mathrm{F}(\mathrm{N}-1, \mathrm{~N}-2)} & \mathrm{F}(\mathrm{N}-1, \mathrm{~N}-1) & \mathrm{F}(\mathrm{N}-1, \mathrm{~N}) & \mathrm{B}(\mathrm{N}-1) \\
\mathrm{F}(\mathrm{N}, \mathrm{N}-1) & \mathrm{F}(\mathrm{N}, \mathrm{N}) & 0 & \mathrm{~B}(\mathrm{~N})
\end{array}\right)
$$

Upon exit from IBTM, the array $X$ in common IBTMN contains the generated solution to the system of linear equations.

Called from:

PLASIM [ENTRY PLASMA]

Variables required:

A - contains the nontrivial information of the block tridiagonal coefficient matrix for the linear system of differenced equations obtained from the set of partial differential equations; also contains the right-hand side

$\mathrm{N}$ - number of radial mesh points

$\mathrm{K}$ - number of partial differential equations describing the plasma Arrays changed:

\footnotetext{
A - destroyed

$X$ - solution vector, at the new time step, of the plasma variable profiles
} 


\section{REFERENCES}

1. R. D. Hazeltine and F. L. Hinton, Phys. Fluids 16: 1883 (1973).

2. M. M. Widner and R. A. Dory, An Implicit Numerical Method for Simulating Tokamak Plasma Discharges, ORNL/TM-3498 (July 1971). 


\section{DESCRIPTION OF NEUTRAL GAS MODULE}

\subsection{PURPOSE AND STRUCTURE OF MODULE}

The neutral gas module models the effects of neutral gas atoms in the plasma. Two distinct species of these atoms are present: hot and cold. The cold neutrals diffuse into the interior of the plasma from the area next to the container wall. When a cold neutral collides with a plasma ion, it can scatter elastically or charge exchange. The result of either process is production of a cold fon and a hot neutral. The purpose of this module is to determine the density distribution of hot and cold neutrals in the plasma. A calculation of the charge-exchange spectrum is included to allow comparison with experiment.

Four approaches are available for modeling the neutral gas effects: 1) an analytic expression based on a series of calculations using a detailed slab model, 2) a detailed slab model calculation without wall reflections, ${ }^{1}$ 3) a slab model with wall reflections, ${ }^{2}$ and 4 ) a Monte Carlo transport code calculation (NUTRLSN). ${ }^{3}$ The analytic model options will always be available in the program. The remaining three options cannot be simultaneously linked together to form the object module of the entire simulation program. This restriction exists to reduce core memory requirements. One of options 2-4 must be selected and the subroutines for that option linked with the rest of the program. This linkage is accomplished on IBM $360 / 370$ series computers by inserting an INCLUDE card with a reference to the name of the cataloged data set containing the subroutines for the desired option as input to the link step. Until options 3 and 4 have been implemented, option 2 will be assumed to be the only one available in the neutral gas module. Figure 5 shows the structure of the subroutine calls for this option. Figure 6 shows the slab geometry used in constructing the model. The user is reminded that the data required for the detailed slab model (DSM) calculations, as well as most of the data generated by the calculations, are communicated to the various subroutines through the labeled commons displayed in Table 10 . 


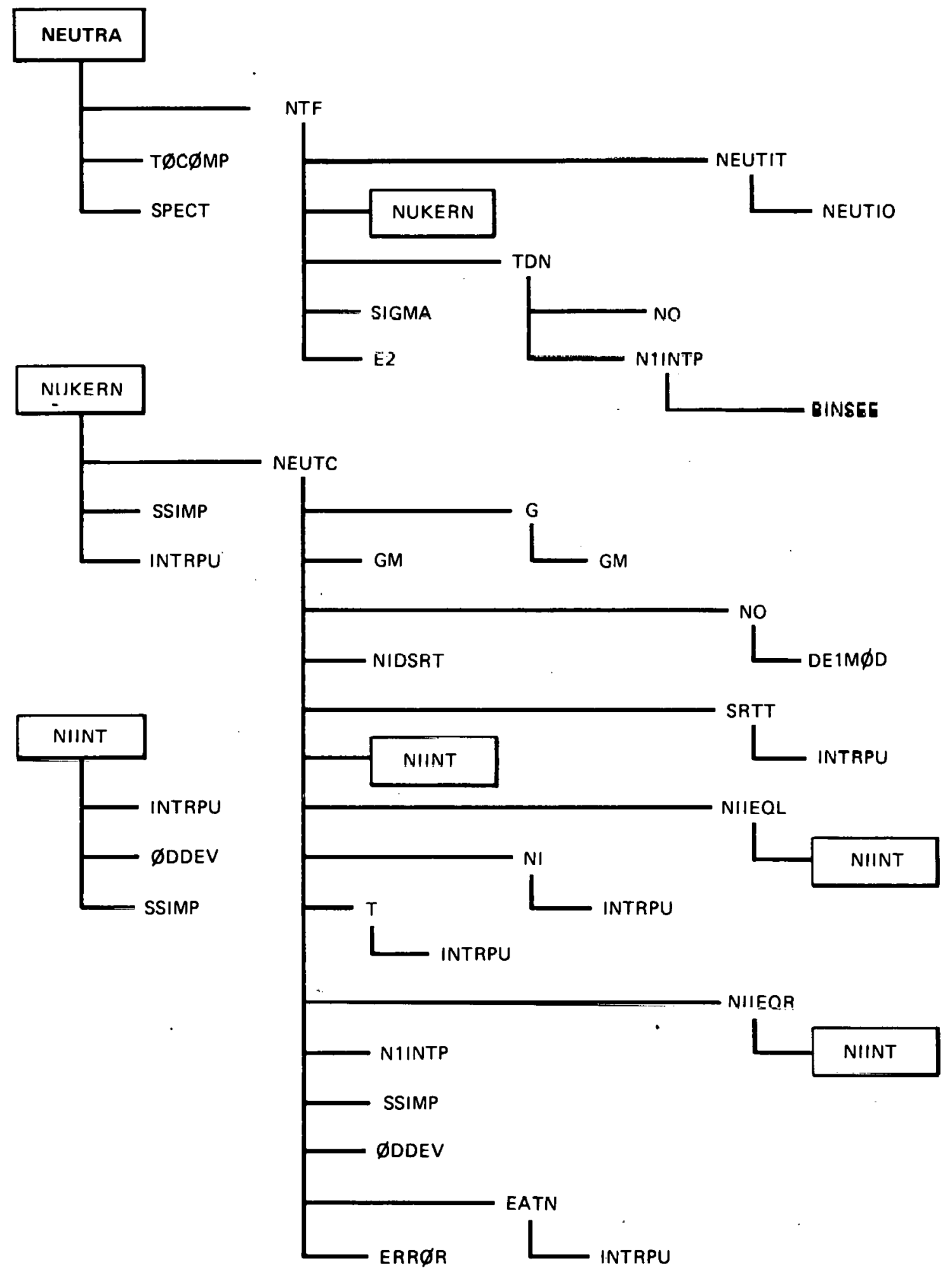

Fig. 5. Diagram of neutral gas module subroutine calls. 


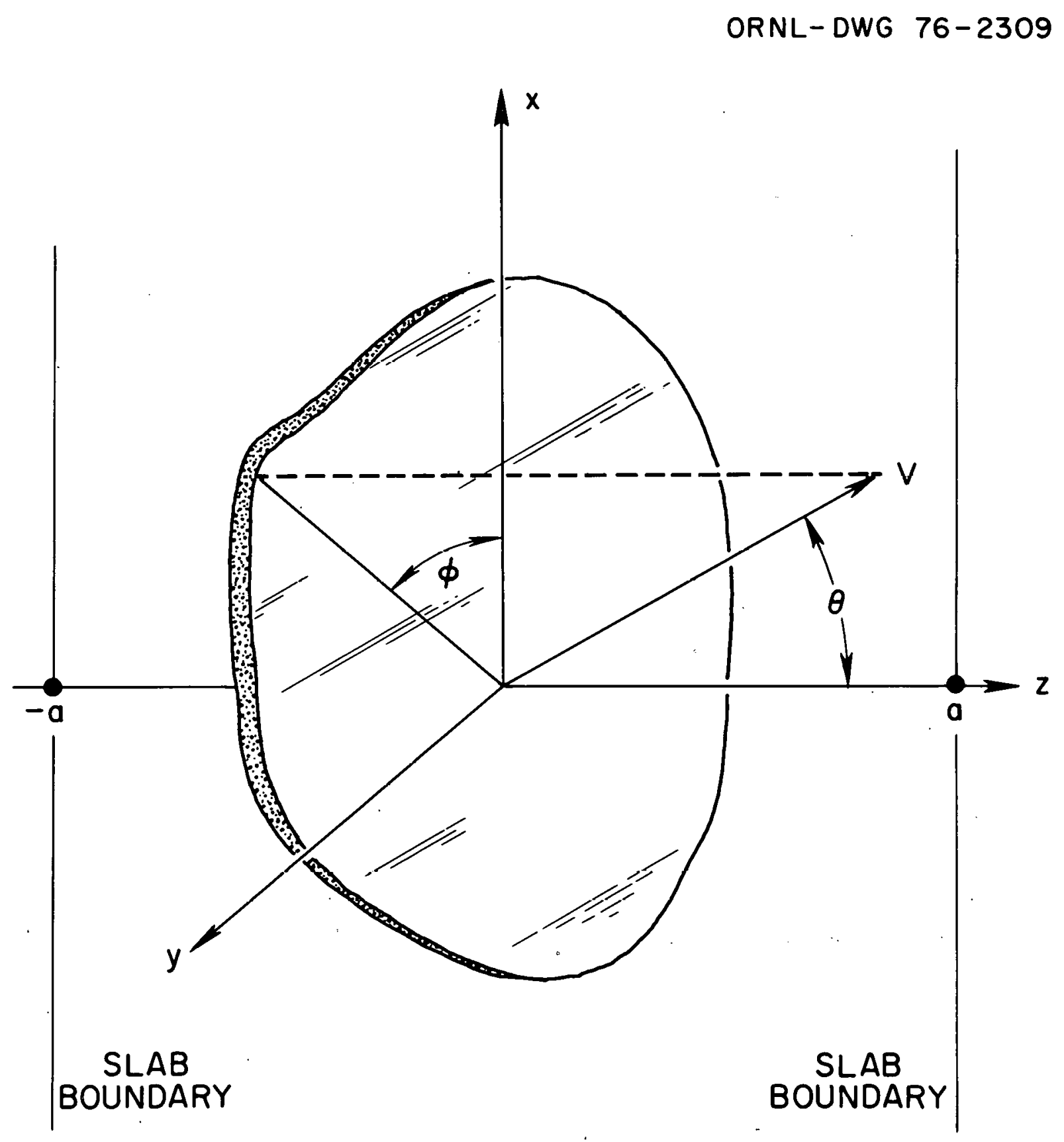

F1g. 6. Slab geometry used in detalled model of neutral gas diffusion and charge exchange. 
Table 10. List of labeled commons and their contents which are used in the detailed slab model (DSM) calculation

CØMM $\emptyset N / N U T R L P / ~ N \emptyset Z, N \emptyset X, N S I M P, N U N I F, Q F, Q C, D Z, D Z D 2, D Z D 3$, EPSN1, 1SIMPM1, BDSQTX, TCINT, N $\varnothing \mathrm{XM1}, \mathrm{N} \emptyset \mathrm{XP1}, \mathrm{N} \emptyset \mathrm{ZP1}$, NSC, ININ, ITIN, IN1, IEATN, 2IINIOX, IINIX1, IX, IZ, IJL, IJH, IIJ, IRAT, NWDW1, MWDSC, 3N, ANM1, DSP, A, B, C, W1 (1) CØMM $\emptyset$ /INTRPS/ INTRPS, NSMO, NSMO21, IDEBUG CØMM $\emptyset$ N/NEUTPT/ contains pointers that partition the array WSC CØMM $\emptyset \mathrm{N} / \mathrm{PRNT} /$ IPA, T.PNO CØMM $\emptyset$ /SLAB/ E $\emptyset$, EPSNO, NWDWSC

\subsection{BASIC INPUT/OUTPUT}

The neutral gas module requires a great deal of input related to the numerical algorithms used to perform the integrations and to obtain the neutral profiles specified by the choice of model. Once set, most of this input should not need changing. This is the reason for setting many of. these values in a BL $\phi C K$ DA'l'A section of the program rather than reading them in every time. The physics input includes the electron and ion temperatures and densities and the energy and dens1cy of the Franck-Cundun neutrals at the plasma surface. The neutral gas module generates the profiles of the hot, cold, and total neutral densities and a total neutral density temperature. A subroutine SPECT computes a charge-exchange spectrum and any other quantities which can be related to experimental measurements and can be computed in terms of the variables appearing in the models used. Subroutine SPECT will most likely be modified by the user to reflect the type of experimental measurements and apparatus used for plasma diagnostics designed to obtain information on neutral gas effects.

\subsection{DESCRIPTION OF THE ANALYTIC MODEL FOR THE TOTAL NEUTRAL DENSITY}

The analytic model is based on the results of a number of cases run using the detailed slab model. ' Let 


$$
\operatorname{DMAX}=10^{-13} \max \left\{10^{11}, \underset{\mathrm{x}}{\max }\left\{\mathrm{N}_{\mathrm{e}}(\mathrm{x})\right\}\right\}
$$

and

$$
\mathrm{ATT}=\frac{85.4 \mathrm{~T}_{i}(0)}{200(\mathrm{DMAX} * \mathrm{AM})^{2}}
$$

where $T_{i}(0)$ is the ion temperature at the center, $\mathrm{N}_{e}$ the electron density, and $A M$ the minor radius of the torus. Then the analytic approximation, $\overline{\mathrm{N}}_{\mathrm{T}}$, to the total neutral density is given by

$$
\overrightarrow{\mathrm{N}}_{\mathrm{T}}(\mathrm{X})=.5 *\left(\operatorname{ATT}\left(1+2 * \mathrm{X}^{\mathrm{NUTE}(2)}\right)+\mathrm{E}_{2}(\mathrm{ZZ})\right)
$$

where

$$
\mathrm{ZZ}=.25 * \mathrm{DMAX} * \mathrm{AM} *(1-\mathrm{X})\left(1-\left(1+\mathrm{X}+\mathrm{X}^{2}\right) / 3\right)
$$

and $E_{2}$ is the exponential integral of the second kind. NUTE(2) is a user-specified input value.

The scaled, total neutral density is obtained by multiplying $\overline{\mathrm{N}}_{\mathrm{\prime}}$, by the Franck-Condon boundary density $\mathrm{N}_{\mathrm{FC}}$ where

$$
\mathrm{N}_{\mathrm{FC}}=(\mathrm{FCDEN}-\mathrm{FCF}) * \mathrm{EXP}(-\mathrm{TIM} / \mathrm{TP} \emptyset)+\mathrm{FCF}
$$

TIM is the current time and FCDEN, FCF, and TP $\emptyset$ are input parameters.

4.4. SLAB MODEL APPROXIMATION TO THE NEUTRAL GAS DENSITY WITHOUT WALL REFLECTION

4.4.1 Neutral Gas Density as the Solution to an Integral Equation

The total neutral gas density, $\mathrm{N}_{\mathrm{T}}$, will be taken as the solution to the integral equation

$$
\mathrm{N}_{\mathrm{T}}(\mathrm{X})=\mathrm{N}_{0}(X)+\mathrm{A} \int_{-1}^{1} \mathrm{~d} z \frac{\tilde{\mathrm{N}}_{\mathrm{i}}(\mathrm{z})}{\sqrt{\ddot{\mathrm{T}}_{\mathrm{i}}(\mathrm{z})}} \mathrm{N}_{\mathrm{T}}(\mathrm{z}) \mathrm{G}(\mathrm{q}(\mathrm{z}, \mathrm{x}))
$$

where $\mathrm{N}_{i}$ is the proton density, $\mathrm{N}_{\mathrm{e}}$ the electron density, $\mathrm{T}_{i}$ the fon temperature, and $\mathrm{T}_{\mathrm{e}}$ the electron temperature, and where 


$$
\begin{aligned}
& \tilde{N}_{i}(z)=N_{i}(z) / N_{i}(0), \\
& \tilde{T}_{i}(z)=T_{i}(z) / T_{i}(0), \\
& q(z, x)=\frac{B}{\sqrt{\tilde{T}_{i}(z)}}\left|\int_{z}^{x} d z^{\prime} \operatorname{EATN}\left(z^{\prime}\right)\right|, \\
& G(q)=\int_{0}^{\infty} d y y e^{-y^{2}} E_{1}(q / y \bar{y}), \\
& \mathrm{N}_{\mathrm{o}}(\mathrm{x})=.5\left(\mathrm{E}_{2}\left(\xi_{1}\right)+\mathrm{E}_{2}\left(\xi_{2}\right)\right) \text {, } \\
& \xi_{1}=c \int_{-1}^{x} d z^{\prime} \operatorname{EATN}\left(z^{\prime}\right) \\
& \xi_{2}=\mathrm{C} \int_{\mathrm{x}}^{1} \mathrm{~d} z^{\prime} \operatorname{EATN}\left(z^{\prime}\right), \\
& \operatorname{EATN}(x)=\frac{N_{i}(x) \sigma_{C X}(x) \sqrt{T_{i}(x)}+N_{e}(x) \sigma_{E L}(x) \sqrt{1836.15152 T_{e}(x)}}{N_{i}(0) \sigma_{C x}(0) \sqrt{T_{i}(0)}}, \\
& A=1.13(\mathrm{AM}) \sigma_{\mathrm{cx}}(0) \mathrm{N}_{i}(0), \\
& B=A, \\
& C=A \sqrt{T_{i}(0) / E \emptyset} \text {. }
\end{aligned}
$$

Moreover, $\sigma_{\mathrm{cx}}$ is the cross section for charge exchange between atomic neutral hydrogen and protons; $\sigma_{E L} i \varepsilon$ the cross section for ionization of atomic neutral hydrogen by electron impact; AM is the minor radius of the torus; and $E \emptyset$ is the external gas energy.

The computational procedure actually approximates the hot neutral density $N_{1} \cdot N_{1}$ is equal to $N_{T}-N_{0}$ and $N_{1}$ satisfles the integral equation

$$
N_{1}(x)=A \int_{-1}^{1} d z \frac{\tilde{N}_{i}(z)}{\sqrt{\tilde{T}_{i}(z)}}\left[N_{0}(z)+N_{1}(z)\right] G[q(z, x)] .
$$


4.4.2 Computational Procedure for the Determination of an Approximation to the Hot Neutral Density, $\mathrm{N}_{1}$

For $\mathrm{x}$ in $[0,1]$

$$
\gamma(x) \equiv A \int_{-1}^{1} d z \frac{\tilde{\mathrm{N}}_{i}(z)}{\sqrt{\tilde{\mathrm{T}}_{i}(z)}} G(q(z, x))
$$

If

$$
\bar{\gamma} \equiv \max \{\gamma(\mathrm{x})\}<1,
$$

then the method of functional iteration will yield a sequence of functions $\left\{\mathrm{N}_{1}{ }^{(i)}\right\}$ that converges to the function $N_{1}$. In fact, let $N_{l}(0)$ be an arbitrary continuous function. Then for $x$ in $[0,1]$ and for $i=1,2$, ..., define

$$
\begin{gathered}
N_{l}(i+1)(x) \equiv \hat{I}\left(N_{l}(i), x\right) \equiv A \int_{-1}^{1} d z \frac{\tilde{N}_{i}(z)}{\sqrt{\tilde{T}_{i}(z)}}\left[N_{0}(z)\right. \\
\left.+N_{l}{ }^{(i)}(z)\right] G(q(z, x)) .
\end{gathered}
$$

Then

$$
\begin{aligned}
& \max _{x}\left\{\left|N_{1}(x)-N_{1}{ }^{(i)}(x)\right|\right\} \leqslant\left[\frac{\bar{\gamma}}{1-\bar{\gamma}}\right] \max _{x}\left\{\left|N_{l}{ }^{(i)}(x)-N_{l}^{(i-1)}(x)\right|\right\} \\
& \leqslant\left[\frac{\bar{\gamma}}{1-\bar{\gamma}}\right]_{x} \max _{x}\left\{\left|N_{1}^{(1)}(x)-N_{1}^{(0)}(x)\right|\right\} .
\end{aligned}
$$

If $\bar{\gamma} \geqslant 1$, then the method of functional iteration is, in general, inappropriate. This does not necessarily mean that the integral equation has no solution.

The discrete analogue of the continuous problem expressed by Eq. (3) employs a finite partition of the unit interval $[0,1]$. Let

$$
0=x_{1}<x_{2}<\ldots<x_{N \emptyset x}=1
$$

be the $N \emptyset \mathrm{X}$ nodes at which an approximation to the hot neutral density, $N_{1}\left(x_{j}\right)$, is desired. The approximation to $N_{l}\left(x_{j}\right)$ is taken to be an approximation to $\hat{\mathrm{N}}_{1}\left(\mathrm{x}_{j}\right)$ where 


$$
\hat{N}_{1}\left(x_{j}\right)=A \int_{-I}^{1} d z \frac{\tilde{N}_{i}(z)}{\sqrt{\tilde{\mathrm{T}}_{i}(z)}}\left[N_{0}(z)+\hat{\mathrm{N}}_{1}(z)\right] G\left[q\left(z, x_{j}\right)\right]
$$

and $\hat{\mathrm{N}}_{1}$ is a continuous, piecewise linear function on $[0,1]$ with nodes at $x_{j}$. The definition of $\hat{\mathrm{N}}_{1}$ is extended to $[-1,0]$ by the requirement that $\hat{\mathrm{N}}_{1}$ be symmetric with respect to $\mathrm{x}=0$.

The computation defines

$$
\hat{\mathrm{N}}_{1}{ }^{(0)}\left(\mathrm{x}_{\mathrm{j}}\right)=0 \quad \mathrm{j}=1, \ldots, \mathrm{N} \varnothing \mathrm{x}
$$

and

$$
\hat{\mathrm{N}}_{1}{ }^{(i+1)}\left(\mathrm{x}_{j}\right)=\hat{\mathrm{I}}\left(\hat{\mathrm{N}}_{1}{ }^{(i)}, \mathrm{x}_{j}\right) \quad \mathrm{j}=1, \ldots, \mathrm{N} \emptyset \mathrm{X}
$$

where $\hat{\mathrm{I}}$ is defined in Eq. (6). The definition of $\hat{\mathrm{N}}_{1}{ }^{(i)}$ for $x$ not equal to $x_{j}$ is determined by the requirement that $\hat{N}_{l}{ }^{(i)}$ be a continuous, piecewise linear function on $[0,1]$ with nodes at $x_{j}$. The 1nequality given in Eq. (7) is valid for the discrete case if $\hat{\mathrm{N}}_{1}$ is identified with $\mathrm{N}_{1}$ and if

$$
\hat{\gamma}=\max \left\{\gamma\left(x_{j}\right)\right\}
$$

is identified with $\bar{\gamma}$. For fixed $j$ the sequence $\left\{\hat{\mathrm{N}}_{1}{ }^{(i)}\left(\mathrm{x}_{j}\right)\right\}$ converges monotonically from below to $\hat{\mathrm{N}}_{1}\left(\mathrm{x}_{j}\right)$ whenever $\hat{\gamma}<1$.

It can be shown $(\hat{\gamma}<1)$ that

$$
\left|N_{1}\left(x_{j}\right)-\hat{N}_{1}\left(x_{j}\right)\right| \leqslant \gamma \varepsilon\left(1+\frac{\hat{\gamma}}{1-\gamma}\right)
$$

where

$$
\varepsilon=\max _{x}\left\{\left|N_{l}(x)-N_{l}^{*}(x)\right|\right\}
$$

and $\mathrm{N}_{1}{ }^{*}$ is the continuous, piecewise linear function with nodes at $x_{j}$ that satisfies

$$
N_{l}^{*}\left(x_{j}\right)=N_{i}\left(x_{j}\right)
$$




\subsubsection{Computational Description of the Approximation to $\hat{I}\left(g, x_{j}\right)$}

The basic computational problem is to make an adequate approximation of the value of the integral representation for $\hat{I}\left(g, x_{j}\right)$ as expressed in $\mathrm{Eq}$. (6) where $\mathrm{g}$ is a continuous, piecewise linear function on $[0,1]$ with nodes $\left\{x_{j}\right\}$. The definition of $g$ is extended to $[-1,0]$ by the requirement of symmetry with respect to $x=0$. A straightforward quadrature formula is not adequate since the function $G$ in $\mathrm{Eq}$. (2) possesses a logarithmic singularity at $q=0 ; G$ may be represented by

$$
G(q)=-\frac{\ln q}{2}+G M(q)
$$

where GM is continuous for $q \geqslant 0$. Let

$$
\mathrm{U}(\mathrm{z}, \mathrm{x})=\left|\int_{\mathrm{z}}^{\mathrm{X}} \mathrm{d} \mathrm{z}^{\prime} \operatorname{EATN}\left(\mathrm{z}^{\prime}\right)\right|
$$

Then $G$ can be expressed by

$$
G[\mathrm{q}(\mathrm{z}, \mathrm{x})]=-\frac{\ln U(\mathrm{z}, \mathrm{x})}{2}+\left\{0.25 \ln \left(\frac{\tilde{\mathrm{T}}_{i}(\mathrm{z})}{\mathrm{B}^{2}}\right)+\mathrm{GM}[\mathrm{q}(\mathrm{z}, \mathrm{x})]\right\} \text {. }
$$

The integrand in the integral representation for $\hat{I}\left(g, x_{j}\right)$ possesses a logarithmic singularity at $z=x_{j}$. The situation may be handled by partitioning the integration interval as follows:

$$
\begin{aligned}
\int_{-1}^{1}=\int_{-1}^{m^{-}(Q C)}+\int_{m^{-}(Q C)}^{n^{-}(Q F)}+\int_{n^{-}(Q F)}^{x_{j}}+\int_{x_{j}}^{n^{+}(Q F)}+\int_{n^{+}(Q F)}^{m^{+}(Q C)} \\
+\int_{m^{+}(Q C)}^{1}
\end{aligned}
$$

Let

$$
-1=z_{1}<z_{2}<\ldots<z_{N \phi Z}=1
$$

be a uniform partition of the interval $[-1,1]$ and let $N \emptyset Z$ be odd. Moreover, let 


$$
0 \leqslant Q F<Q C
$$

be two fixed constants. Then define for each $x_{j}$

$$
\begin{aligned}
& \mathrm{m}^{-}(Q C)=\max \left\{z_{i} \text { such that } z_{i}<x_{j} \text { and } q\left(z_{i}, x_{j}\right) \geqslant Q C\right\}, \\
& \mathrm{n}^{-}(Q F)=z \text { such that } z<x_{j} \text { and } q\left(z, x_{j}\right)=Q F, \\
& \mathrm{n}^{+}(Q F)=z \text { such that } z>x_{j} \text { and } q\left(x_{j}, z\right)=Q F, \\
& m^{+}(Q C)=\min \left\{z_{i} \text { such that } z_{1}>x_{j} \text { and } q\left(x_{\cdot j}, z_{i}\right) \geqslant Q C\right\} .
\end{aligned}
$$

Undefined intervals are determined and handled correctly. The first three subintervals and the last three are qualitatively the same. If an odd number of $z_{i}$ nodes are in $\left[-1, \mathrm{~m}^{-}(Q C)\right]$, then a Simpson's approximation to the integral over $\left[-1, \mathrm{~m}^{-}(Q C)\right]$ is calculated with these nodes. If there are an even number of $z_{i}$ nodes, then a trapezoidal approximation is made with the first two nodes and a Simpson's approximation on $\left[z_{2}, m^{-}\right.$ $(Q C)]$. A Simpson!s approximation to the integral over $\left[m(Q C), n^{-}(Q F)\right]$ is calculated using NSIMP uniformly distributed nodes. To handle the third integral on the right-hand side of $\mathrm{Eq}$. (19) the integrand is decomposed into a sum. The derivative of $U$ with respect to $z$ is given by [see Eq. (17) ]

$$
\begin{aligned}
& U^{\prime}(z, x)=-\operatorname{EATN}(z), \quad z<x \\
& U^{\prime}(x, z)=\operatorname{EATN}(z), \quad z>x
\end{aligned}
$$

Using this and the representation for G expressed in Eq. (18), the third integral may be written as

$$
\begin{gathered}
\int_{n-(Q F)}^{x_{j}} d z \frac{\tilde{N}_{i}(z)}{\sqrt{\tilde{T}_{i}(z)}}\left[N_{0}(z)+g(z)\right]\left\{0.25 \ln \left(\frac{\tilde{I}(z)}{B 2}\right)+G M\left[q\left(z, x_{j}\right)\right]\right\} \\
\quad+0.5 A \int_{n^{-}(Q F)}^{x_{j}} d\left\{U\left(z, x_{j}\right)\left[\ln U\left(z, x_{j}\right)-1\right]\right\} h(z)
\end{gathered}
$$

where

$$
h(z)=\frac{N_{0}(z)+g(z)}{\sqrt{\tilde{T}_{i}(z)}} \frac{\tilde{N}_{i}(z)}{\operatorname{EATN}(z)}
$$


Let

$$
\mathrm{n}^{-}(\mathrm{QF})=\mathrm{y}_{1}<\mathrm{y}_{2}<\ldots<\mathrm{y}_{\mathrm{NSMO}}=\mathrm{x}_{\mathrm{j}}
$$

be a uniform partition of the interval $\left[\mathrm{n}^{-}(\mathrm{QF}), \mathrm{x}_{j}\right]$ where NSMO is odd and $\geqslant 3$. Then the first integral in Eq. (24) is approximated by Simpson's rule with the nodes $y_{i}$; the second integral is approximated by

$$
0.25 \mathrm{~A} \sum_{k=1}^{\mathrm{NSMO}-1}\left[\mathrm{~h}\left(\mathrm{y}_{\mathrm{k}}\right)+\mathrm{h}\left(\mathrm{y}_{\mathrm{k}+1}\right)\right]\left[\mathrm{s}_{\mathrm{k}+1}\left(\ln \mathrm{s}_{\mathrm{k}+1}-1\right)-\mathrm{s}_{\mathrm{k}}\left(\ln \mathrm{s}_{\mathrm{k}}-1\right)\right]
$$

where

$$
s_{k}=U\left(y_{k}, x_{j}\right)
$$

\subsubsection{Required Input, Space Allocation, and Calling Sequence for Computer Program}

With the input specified and with sufficient memory space in the arrays W1 and WSC, the calls

CALL NEUTIT

CALL NUKERN (WSC, NPR ØFI)

will yield, if $\hat{\gamma}<1$, an approximation to $\hat{\mathrm{N}}_{1}\left(\mathrm{x}_{j}\right)$. In fact

$$
W I(\operatorname{INI}+J)=\hat{N}_{I}{ }^{\left(i_{i}\right)}\left(x_{j}\right)
$$

and the FØRTRAN function TDN may be used to give the approximation

$$
\mathrm{N}_{0}(\mathrm{x})+\hat{\mathrm{N}}_{1}^{(\mathrm{i})}(\mathrm{x})
$$

to $\mathrm{N}_{\mathrm{T}}(\mathrm{x})$ where $\hat{\mathrm{N}}_{1}(i)$ is the $i$-th continuous, piecewise linear function defined by Eq. (11) and $x$ is in $[-1,1]$. The value of $i$ is determined by the accuracy desired in the approximation; 1 is the least $i$ for which

$$
\begin{aligned}
\max _{x_{j}}\left\{\mid \hat{N}_{1}\left(x_{j}\right)\right. & \left.-\hat{N}_{1}^{(i)}\left(x_{j}\right) \mid\right\} \leqslant\left[\frac{\hat{\gamma}}{1-\hat{\gamma}}\right] \max _{j}\left\{\mid \hat{N}_{1}^{(i)}\left(x_{j}\right)\right. \\
& \left.-\hat{N}_{1}^{(i-1)}\left(x_{j}\right) \mid\right\} \leqslant \text { EPSN } 1
\end{aligned}
$$


where EPSN 1 is user specified. NUKERN sets NPR $\emptyset$ FI to 0 if a new profile has been generated and to 1 if no new profile has been generated.

Prior to the CALL NEUTIT the values for the variables $N, N \phi X, N \phi Z$, NSIMP, NSMO, NUNIF, QF, QC, EPSN1, INTRPS, and IDEBUG must be specified. However, QF, QC, EPSN1, INTRPS, and IDEBUG may be changed subsequently without issuing another CALL NEUTIT. Moreover, subsequent $\mathrm{N}_{\mathrm{T}}$ profiles may be generated without another CALL NEUTIT unless a value of $N, N \emptyset X$, NøZ, NSIMP, NSMO, or NUNIF has changed.

Let

$$
y_{L}=(L-1) /(N-1) \quad L=1, \ldots, N \quad \text {. }
$$

Prior to the CALL NUKEKN the tollowing variables must be defined lsee Eq. (2)]:

$$
\begin{array}{ll}
W I(\operatorname{ININ}+\mathrm{L})=\tilde{\mathrm{N}}_{i}\left(\mathrm{y}_{\mathrm{L}}\right), & \ldots \\
\mathrm{WI}(\operatorname{ITIN}+\mathrm{L})=\tilde{\mathrm{T}}_{i}\left(\mathrm{y}_{\mathrm{L}}\right), \quad \mathrm{L}+1, \ldots, N \\
\mathrm{WI}(\operatorname{IEATN}+\mathrm{L})=\operatorname{EATN}\left(y_{L}\right),
\end{array}
$$

and $A, B$, and $C$.

Except for WSC and NPR $\emptyset$ FI all the FØRTRAN variables and arrays appear in the labeled commons 1isted in Table 10.

'he array WI must have a minimum of

$5 N+4 N \varnothing \mathrm{X}+3 \mathrm{~N} \varnothing \mathrm{Z}$

elements in its definition. Moreover, the number of elements in the array Wl must be specified in the variable NWDWl in common NUTRLP. It is convenient to dimension $W 1$ and define NWDWI in a BLøCK DATA subprogram. In this way the size of W1 can easily be set to the value in Eq. (34).

The first argument in the parameter list for NUKERN is a scratch array to be used-by NUKERN. This array must have at least $(Q F>0)$

$$
\mathrm{N} \emptyset \mathrm{Z}(\mathrm{N} \emptyset \mathrm{Z}+10 \mathrm{NSMO}+5)+\mathrm{NSIMP}(8 \mathrm{~N} \emptyset \mathrm{X}+1)+4(\mathrm{~N} \emptyset \mathrm{Z}+\mathrm{NSMO})-2
$$

elements in its definition. NEUTIT places the required number of elements in WSC in MWDSC in common NUTRLP. The user should check that WSC has at least MWDSC elements prior to the call to NUKERN. 
A fuller understanding of the input parameters may be gained by reviewing the previous section and Sect. 1.5.

\subsection{SUMMARY OF FUNCTION OF EACH SUBROUTINE}

The descriptions of the subroutines appearing in this section are grouped as much as possible according to the model with which they are associated. Subroutines used in common by two or more of the models are in the group which appears first and which includes the executive subroutines for this module. 
SUBRøUTINE NEUTRA

A call to NTF returns to NEU'LRA the estimate of the total neutral density in the local array XTR. This is then scaled by the Franck-Condon boundary density FCDEX and placed in the array ZNI for export through a labeled common. In fact

$\mathrm{FCDEX}=(\mathrm{FCDEN}-\mathrm{FCF}) * \operatorname{EXP}(-\mathrm{TIM} / \mathrm{TP} \emptyset)+\mathrm{FCF}$

and

ZN1 $\%$ FCDEX*XTR

where TIM is the current time and FCDEN, FCF, and TP $\varnothing$ are user-specified parameters.

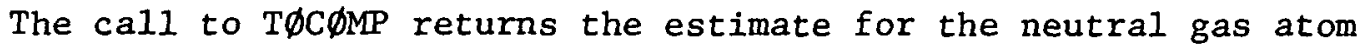
temperature profile $T_{0}$ in the array $T \emptyset$.

Called from: $\quad$ GETSET, MAIN

Subroutines called: NTF, TøC $\emptyset \mathrm{MP}$, SPECT

Commons required: IMPURT, INDEX, IØNS, LBMIBT, NEUTRL, TEMP, TIME

Variables required: FCDEN, FCF, TP $\emptyset$, TIM, N

Arrays required: $\quad$ DEN, DENP, TE, TI, T $\emptyset$

Variables and arrays changed: (See Subroutine NTF)

XTR total neutral density. May not be altered if Nill'f(1) $=2$ or 3 .

ZN1 - total neutral density multiplicatively scaled by the Franck-Condon boundary density.

FCDEX $-=$ NFC = Franck-Condon boundary density

$\emptyset N I D A I$ - contains $N_{i} / \sqrt{T_{i}}$ where $N_{i}$ and $T_{i}$ were used in the ralrulation of the profile in XTR. Applies only when $\operatorname{NUTE}(1)=2$ or 3 . See Eq. (2) in Sect. 4.4

SHG - cemporary sturage ful NTF. Used only if NUTE(1) 2. nr 3. 
SUBRøUTINE NTF(NT, TE, TI, NE, NI, ØNIDAI, NTNEW, WSC)

Subroutine NTF is structured to accommodate and/or drive four models for estimating the total neutral density. The switch NUTE(1) is used to select the model desired. Currentiy only two models are available. If $\operatorname{NUTE}(1)=1$, then the analytic model described in Sect. 4.3 is selected. If $\operatorname{NUTE}(1)=2$, then the slab model approximation (without wall reflection) described in Sect. 4.4 is selected.

Suppose $\operatorname{NUTE}(1)=2$. In order to conserve computing time, the following criterion has been included to determine whether a new neutral density is to be calculated. When a neutral density is determined, the values $\mathrm{N}_{i} / \sqrt{\mathrm{T}_{i}}$ are stored in the array $\emptyset \mathrm{NIDAI}$ and the scalar $\sigma_{c x}(0) \sqrt{\mathrm{T}_{i}(0)}$ in the variable $\mathrm{Cl}$. On successive calls the range of the relative error between the values $C 1 * \emptyset \mathrm{NIDAI}(I)$ and the associated current values is determined. If the magnitude of the relative error for any I exceeds the user-specified parameter EPSNO, then a new neutral profile is requested. However, if NPR $\emptyset \mathrm{FI}=1$ (see Sect. 4.4.4), then the most recently generated neutra1 profile will be used. In the case where NPR $\emptyset F I=1$ on the first ca.1. to NUKERN, the analytic model is used to generate a neutral profile.

The variables described in Eq. (33) are defined within NTF whenever $\operatorname{NUTE}(1)=2$.

Called from:

NEUTRA

Subroutines called: NEUTIT, NUKERN, TDN, SIGMA, E2

Commons required: GE $\emptyset$, INDEX, NUTRLP, PRNT, SLAB, TIME

Variables and arrays changed:

NT - neutral gas density to use with given profiles

$\emptyset \mathrm{NIDAI} *=\mathrm{NI}(\mathrm{I}) / \mathrm{SQRT}(\mathrm{TI}(\mathrm{I}))$ for the most recently calculated neutral profile

NTNEW* = scratch array with $\mathrm{N}$ elements

WSC* = scratch array with at least the number of elements as given in Eq. (35).

$\mathrm{Cl*}=\sigma_{\mathrm{cx}}(0) \sqrt{\mathrm{T}_{1}(0)}$ for the most recent1y calculated ncutral profile.

*Applicable only when $\operatorname{NUTE}(1)=2$. 
SUBR $\emptyset \mathrm{UTINE} T \emptyset \mathrm{C} \emptyset \mathrm{MP}(\mathrm{T} \emptyset, \mathrm{TI})$

The various models for estimating the neutral gas atom temperature $\mathrm{T}_{0}$ should be contained in subroutine $\mathrm{T} \emptyset \mathrm{C} \emptyset \mathrm{MP}$ or driven from it. Presently the only model is to take $\mathrm{T}_{0}$ to be one tenth of $\mathrm{T}_{i}$ where $\mathrm{T}_{i}$ is the ion temperature.

$$
T \emptyset(I)=. I * T I(I) \quad I=1, \ldots, N
$$

Called from: $\quad$ NEUTRA

Commons required: INDEX

Array changed:

$T \emptyset$ - upon exit $T \emptyset$ contains the estimate for $T_{0}$ at the usual grid puluLs, 
SUBRØUTINE SPECT

The subroutine must presently be user supplied since it compares simulated results with measured values and thus depends on the particular experimental apparatus and instrumentation used to obtain the measured values (see Sect. 4.2). 
SUBRØUTINE NEUTIT

Subroutine NEUTIT defines the pointers that logically partition the arrays $W 1$ in common NUTRLP and in WSC, the scratch array that will be passed to NUKERN. Prints most of the level 0 data and checks that $\mathrm{NWDWL} \geqslant 5 \mathrm{~N}+4 \mathrm{~N} \phi \mathrm{X}+3 \mathrm{~N} \phi \mathrm{Z}$.

If this inequality is not satisfied, a message is printed and a CALL EXIT is issued. If the inequality is satisfied, then NEUTIO is called before returning.

Called frow:

NTF

Subroutines called: NEUTIO, possibly EXIT

Commons required: NUTRLP, NEUTPT, INTRPS

Variables required: Level 0 data, i.e., N, N $\varnothing \mathrm{X}, \mathrm{N} \emptyset \mathrm{Z}$, NSIMP, NSMO, NUNIF, QF, QC, EPSN1, INTRPS, IDEBUG. If NUNIF $\neq 0$, values to use for $x_{j}$ must be in $W I(J)$. Level 0 data is stored in commons NUTRLP and INTRPS. 
SUBRØUTINE NEUTIO

Subroutine NEUTIO basically defines arrays that expedite the calculation of $\hat{\mathrm{N}}_{l}{ }^{(i)}\left(z_{j}\right)$ where the $z_{j}$ is defined in Eq. (20) and in arrays of $\mathrm{Z}$ subscripts to aid in the determination of $\mathrm{m}^{-}(\mathrm{QC})$ and $\mathrm{m}^{+}(\mathrm{QC})$ in Eq. (22).

Called from:

NEUTIT

Subroutines called: $\operatorname{EXIT}$ (whenever $\mathrm{X}(1) \neq 0$ or $\mathrm{X}(\mathrm{N} \emptyset \mathrm{X}) \neq 1$ )

Commons required: NUTRLP

Variables required: $\quad \mathrm{N} \emptyset \mathrm{x}, \mathrm{N} \emptyset \mathrm{Z}, \mathrm{NSIMP}, \mathrm{NUNIF}, \mathrm{QF}, \mathrm{QC}, \mathrm{N}$

Variables defined: $\quad$ SIMPMI $=$ NSIMP-1, ANM1 $=N-1, D S P=1 . /$ ANMI, $\mathrm{N} \emptyset \mathrm{XM} 1=\mathrm{N} \emptyset \mathrm{X}-1, \mathrm{~N} \emptyset \mathrm{XP} 1, \mathrm{~N} \emptyset \mathrm{ZP} 1$

Arrays defined: $\quad \mathrm{Z}, \mathrm{X}, \mathrm{JL}, \mathrm{JH}, \mathrm{T} \cdot \mathrm{J}, \mathrm{RAT}$ 
SUBR ØUTINE NUKERN (WSC, NPR $\emptyset$ FI)

Subroutine NUKERN first generates the tables

$$
\begin{aligned}
& W 1(\operatorname{IINIOX}+I)=\int_{0}^{y_{I}} \mathrm{dZ} \operatorname{EATN}(Z), \\
& W 1(\operatorname{IINIXI}+I)=\int_{y_{I}}^{1} \mathrm{dZ} \operatorname{EATN}(Z)
\end{aligned}
$$

for $I=1, \ldots, N$ where $y_{I}=(I-1) /(N-1)$. These are used in NIINT. Then two calls to NEUTC are issued. The first returns the set of values given in Eq. (12). Then NUKERN computes the Lipschitz constant $\hat{\gamma}$ In Eq. (12). If $\hat{\gamma}<1$ and the number of estimated functional iterations required to obtain the accuracy expressed by EPSN1 is less than MAXIT $(-250)$, then a second call to NEUTC is issued to obtain the desired approximations to $\hat{\mathrm{N}}_{l}\left(\mathrm{x}_{j}\right)$. The user should note that if $\hat{\gamma} \geqslant 1$, the computational procedure may be inappropriate, even though the integral equation may have a solution. If a problem is encountered, the user should review Sect. 4.4.2 on the computational procedure for the determination of an approximation to the neutral density. Facilities to print information from NUKERN are provided through the parameler IDEBUG, discuaocd in sect. 1.5 .

Called from: NTF

Subroutines called: NEUTC, SSIMP, INTRPU, AL $\emptyset \mathrm{G}$

Commons required: NUTRLP, NEUTPT, INTRPS

Variables required:

$$
\begin{aligned}
& W 1\left(\text { ININ+I) }=\tilde{N}_{i}\left(y_{I}\right)\right. \text {, the normalized ion density } \\
& W 1(\text { ITIN }+I)=\tilde{T}_{i}\left(y_{I}\right) \text {, the normalized ion temperature } \\
& \text { W1 (IEATNH }+\mathrm{I})=\operatorname{BATN}\left(\mathrm{y}_{\mathrm{f}}\right) \text {, ectimate of the total neutral gas } \\
& \text { attenuation coefficient at the radial point } y_{I} \text {. } \\
& \text { A } \quad=1.13 * \mathrm{AM}^{*} \sigma_{\mathrm{CX}}(0) * \mathrm{~N}_{1}(0) \\
& \mathrm{B} \quad \mathrm{A} \\
& \text { C } \quad=A^{*} \sqrt{T_{i}(0) / E \emptyset} \text { and a scratch array, wSC, with at } \\
& \text { least MWDSC elements. }
\end{aligned}
$$


SUBR $\emptyset$ UTINE NEUTC

If NSC (in common NUTRLP) $=1$, then

$$
\gamma\left(x_{j}\right)=A \int_{-1}^{1} d z \frac{\tilde{N}_{i}(z)}{\sqrt{\tilde{T}_{i}(z)}} G\left(q\left(z, x_{j}\right)\right)
$$

is approximated for $j=I, \ldots, N \emptyset X$. If $N S C=0$, then approximations to $\hat{\mathrm{N}}_{1}\left(\mathrm{x}_{\mathrm{j}}\right)$ are obtained. See Sect. 4.4.2.

Called from: $\quad$ NUKERN

Subroutines called: G, GM, NO, NIDSRT, SRTT, NIINT, NIIEQL, NI, T, NIIEQR, NIINTP, SSIMP, $\emptyset \mathrm{DDEV}$, EATN, AL $\emptyset \mathrm{G}$, AMAXI, ERR $\emptyset R$ (do not expect ERR $\emptyset R$ to be called)

Commons required: NUTRLP, INTRPS 
FUNCTIØN TDN (X)

$$
\begin{aligned}
& \text { In function } \operatorname{TDN}(\mathrm{X}), \\
& \operatorname{TDN}(\mathrm{X})=\mathrm{N}_{\mathrm{o}}(\mathrm{X})+\hat{\mathrm{N}}_{\mathrm{l}}{ }^{(\mathrm{i})}(\mathrm{X})
\end{aligned}
$$

where the functional iteration [see Eq. (11)] performed in NEUTC terminated with the $i-t h$ iterate. It is assumed that $X$ is in $[-1,1]$.

Called from: $\quad$ NTF

Subroutines called: NO, N1INTP

Commons required: NUTRLP 
FUNCTI $\varnothing \mathrm{N}$ NO (X)

If NSC $\neq 0$, then NO $=1$. This is required in the approximations to the function $\gamma$ at the nodal points $x_{j}$.

If NSC $=0$, then NO is the approximate value of the source term in the integral equation for the total neutral density;

$$
\text { NO }=.5\left(E_{2}(X I 1)+E_{2}(X I 2)\right)
$$

where

$$
X I 1=c \int_{-1}^{X} d z \operatorname{EATN}(z)
$$

and

$$
X I 2=c \int_{X}^{1} d z \operatorname{EATN}(z)
$$

Double precision is used for the evaluation of $(1-X * \operatorname{DEXP}(X) * \operatorname{E} 1(X)) / D E X P(X)$

as the representation for the second exponential integral $\mathrm{E}_{2}$.

Called from: NEUTC

Subroutines called: INTRPU, DE1M $\varnothing \mathrm{D}$, DEXP

Commons required: NUTRLP 
SUBRØUTINE DEIM $\emptyset \mathrm{D}(\mathrm{X}, \mathrm{ZEXPX}, \mathrm{E} 1 \mathrm{XM})$

This subroutine returns $\operatorname{E} 1 X M=X * \operatorname{EXP}(X) * E_{1}(X)$.

Called from: No

Subroutines called: DL $\varnothing \mathrm{G}$

Variables required: $\quad \mathrm{X}, \mathrm{ZEXPX}=\operatorname{DEXP}(\mathrm{X})$ 
FUNCTI

The coordinates

$$
(\mathrm{X}(\mathrm{J}), \quad \mathrm{N} 1 \mathrm{~L}(\mathrm{~J})) \quad \mathrm{J}=1, \ldots, \mathrm{N} \emptyset \mathrm{X}
$$

completely describe the continuous, piecewise linear function NlL.

N1INTP returns N1L(Z). This is simply linear interpolation applied to a table of values.

Called from: $\quad$ NEUTC, TDN

Subroutines called: BINSEE 
SUBRØUTINE BINSEE (L, Z, X)

$X$ is the array of nodes in $[0,1]$ at which the hot neutral density, $\mathrm{N}_{1}$, is to be approximated. These are assumed to increase in value as the index increases. BINSEE determines, by bisection, an L such that $X(L) \leqslant|2| \leqslant x(L+1)$.

It is assumed that $|z| \leqslant 1$.

Called from: N1INTP

Sonmmons required: NU'IRLF 
FUNCTI $\varnothing \mathrm{N}$ E2(X)

Function E2 returns the value of the exponential integral of the second kind

$$
E_{2}(x)=\int_{1}^{\infty} \frac{e^{-x t}}{t^{2}} d t
$$

Called from: NTF

Subroutines called: EI

Variables required:

$\mathrm{X}$ - the argument of the function

Variable changed:

E2 - the value of the integral 
$\underline{\text { FUNCTI } \emptyset \mathrm{N} \text { El }(\mathrm{X})}$

Function El returns the value of the exponential integral of the first kind

$$
E_{1}(x)=\int_{x}^{\infty} \frac{e^{-t}}{t} d t
$$

This routine was obtained from the Argonne Code Center. ${ }^{4}$ The routine assumes $\mathrm{x}>0$.

Called from: E2

Variables required:

$\mathrm{X}$ - the argument of the function, the lower limit of the integral. Variables changed:

E1 - the value of the integral 
FUNCTI ØN G(Q)

This function returns an estimate of $G(Q)=\int_{0}^{\infty}$ dy ye $E_{1}(Q / y)$. For $Q>10$ the function $G$ returns 0 as the estimate for $G(Q)$. This may need to be modified. If there exists a generated $Q>10$, then this is reported from subroutine NUKERN. There should be about four significant figures in $G(Q)$ for $Q \leqslant 10$. The user should refer to the comment cards within FØRTRAN function $G$.

Called from: NEUTC

Subroutines called: GM, AL $\emptyset \mathrm{G}$ 
FUNCTIØN GM(Q)

This function returns an estimate of $G M(Q)=G(Q)-\frac{\ln Q}{2}$. GM requires, but does not check, that $Q$ satisfies $Q \leqslant 1$. An appraisal of the accuracy of the estimate is contained within the function as comment cards.

Called from: NEUTC, G 
FUNCTI $\emptyset \mathrm{N}$ NI (Z)

Function $N I(Z)$ estimates $\tilde{N}_{i}(Z)$ at the radial point $Z$. $\tilde{N}_{i}(Z)$, the normalized ion density at $Z$, is estimated according to the interpolation scheme selected by the switch INTRPS.

Called from: $\quad$ NEUTC

Subroutine called: INTRPU

Commons required: NUTRLP 
FUNCTI $\emptyset \mathrm{N} T(\mathrm{Z})$

Function $T(Z)$ estimates $\tilde{T}_{i}(z)$ at the radial point $z$. $\tilde{T}_{i}(z)$, the normalized ion density at $Z$, is estimated according to the interpolation scheme selected by the switch INTRPS.

Called from: $\quad$ NEUTC

Subroutines called: INTRPU

Commons required: NUTRLP 
FUNCTI $\emptyset \mathrm{N}$ NIDSRT $(Z)$

This function approximates $\tilde{\mathrm{N}}_{i}(\mathrm{Z}) / \sqrt{\tilde{T}_{i}(Z)}$ where $\tilde{\mathrm{N}}_{i}$ and $\tilde{\mathrm{T}}_{i}$ are the normalized ion density and temperature. If INTRPS $=1,2$, or 3 , then linear, quadratic, or cubic interpolation is used to approximate $\tilde{N}_{i}(Z)$ and $\tilde{\mathrm{T}}_{i}(\mathrm{Z})$.

Called from: NEUTC

Functions called: INTRPU, SQRT

Commons required: NUTRLP

Variables changed: NIDSRT 
FUNCTI ØN SRTT(Z)

Function $\operatorname{SRTT}(Z)$ estimates $\sqrt{\tilde{T}_{i}(Z)}$ at the radial point $Z$. $\tilde{T}_{i}(Z)$, the normalized ion temperature at $\mathrm{Z}$, is estimated according to the interpolation scheme selected by the switch INTRPS.

Called from:

NEUTC

Subroutines called: INTRPU, SQRT

Commons required: NUTRLP 
FUNCTI $\emptyset N$ NIINT $(Z, X)$

This function estimates $\quad\left|\int_{z}^{x} \mathrm{~d} z^{\prime} \operatorname{EATN}\left(z^{\prime}\right)\right|$

Called from: $\quad$ NEUTC, NIIEQL, NIIEQR

Subroutines called: INTRPU, $\emptyset \mathrm{DDEV}$, SSIMP

Commons required: INTRPU, ØDDEV, SSIMP

Variables required: $\mathrm{Z}, \mathrm{X}$, ANMI, DSP, (WI (IEATN+I), Wl (IINIOX+I), $\mathrm{W} 1(\operatorname{IINIX} 1+\mathrm{I}), \mathrm{I}=1, \ldots, \mathrm{N})$ 
SUBRØUTINE NIIEQL (ZM, BL, BH, VAL)

This subroutine returns in $\mathrm{ZM}$ an approximate solution of

$$
\frac{B}{\sqrt{\tilde{T}_{i}(z)}} \int_{z}^{B H} d z^{\prime} \operatorname{EATN}\left(z^{\prime}\right)=\operatorname{VAL}
$$

that is known a priori to lie in the interval [BL, BH]. Uniqueness of solution is not guaranteed.

Galled from: $\quad$ NEUTC, SRTT

Subivutines callod: NITNT

Variables required: $\mathrm{BL}, \mathrm{BH}, \mathrm{VAL}$ 
SUBRØUTINE NIIEQR(ZM, BL, BH, VAL)

This subroutine returns in $2 M$ an approximate solution of

$$
\frac{B}{\sqrt{\tilde{T}_{i}(z)}} \int_{B L}^{z z} d z^{\prime} \operatorname{EATN}\left(z^{\prime}\right)=\operatorname{VAL}
$$

that is known a priori to lie in the interval [BL, BH]. Uniqueness of solution is not guaranteed.

Called from: $\quad$ NEUTC

Subroutines called: NIINT, SRTT

Variables required: $\mathrm{BL}$, BM, VAL 
FUNCTI $\emptyset \mathrm{N} \operatorname{EATN}(\mathrm{Z})$

This function estimates the total neutral gas attenuation coefficient at the radial point $\mathrm{Z}$ according to the interpolation scheme selected by the switch INTRPS. The analytic expression for the EATN function may be found in $\mathrm{Eq}$. (2).

Called from: NEUTC

Subroutine called: INTRPU

Commons required: NUTRLP 
SUBRфUTINE SSIMP (S, F, N, HD 3)

Subroutine SSIMP returns in S the Simpson's approximation to $\int_{r_{1}}^{r_{N}} \operatorname{dtf}(t)$ where $r_{i}=r_{1}+(i-1) * h, H D 3=h / 3, F(I)=f\left(r_{i}\right)$, and $N$ is odd. If $\mathrm{N}<3$, then $\mathrm{S}=0$.

Called from: NUKERN, NEUTC, NIINT 
FUNCTI $\emptyset \mathrm{N} \emptyset \mathrm{DDEV}(\mathrm{N})$

Function $\emptyset \mathrm{DDEV}$ returns value 0 if $\mathrm{N}$ is even and 1 if $\mathrm{N}$ is odd. $\emptyset \mathrm{DDEV}$ is an integer-valued function.

Called from: NEUTC, NIINT 
FUNCTI $\emptyset N$ INTRPU $(Y, X, Y$, ANM 1$)$

$\mathrm{Y}(\mathrm{I})$ is an approximation to the function $\mathrm{Y}$ evaluated at $(\mathrm{I}-1) /(\mathrm{N}-1)$ where $I=1, \ldots, N$ and $N$ is the number of spatial grid points. ANMI = N-1. Function INTRPU approximates $Y$ at the argument $X$ by means of linear, quadratic, or cubic interpolation whenever $\operatorname{INTRPS}=1,2$, or 3 respectively. It is assumed that $|\mathrm{X}| \leqslant 1$.

Called from: $\quad$ NUKERN, EATN, SRRT, NIDSRT, NO, NIINT

Commons required: INTRPS 
SUBRфUTINE ERR $\emptyset \mathrm{R}$

Subroutine ERR $\emptyset \mathrm{R}$ calls for a dump of the contents of core memory containing the job which references it and terminates the job. An ASSEMBLER language version of the subroutine is available for users having access to the IBM $360 / 370$ series computers.

Called from: NEUTC 


\section{REFERENCES}

1. J. T. Hogan and J. F. Clarke, J. Nucl. Mat. 53: I (1974).

2. J. F. Clarke and D. J. Sigmar, Proc. 7th European Conf. on Controlled Fusion and Plasma Physics, p. 134 (1975).

3. L. M. Petrie and O. W. Herrman, private communication (February 1976).

4. Donald F. Jordan, ANL Subroutine Library Documentation Vol. 2, Sect. C. Polynomials and Special Functions, ANL C369S: DEøNE (January 1973). 


\section{THIS PAGE}

\section{WAS INTENTIONALLY \\ LEFT BLANK}




\section{DESCRIPTION OF THE IMPURITY MODULE}

\subsection{PURPOSE AND STRUCTURE OF MODULE}

The impurity module calculates the properties of nonhydrogenic ions in the plasma. Subroutine IMPRTY directs the choice of models for stripping of the multicharged ions, for spatial diffusion according to the transport model selected, and for radiative loss. The module requires the choice of a simple description [IMP(3) =1] or a detailed model $[\operatorname{IMP}(3)=2]$, based on one of the options selected with the switch IMP(2), for carbon, oxygen, and a pseudo "high-Z" or iron impurity.

Impurity stripping is currently modeled using corona equilibrium tables [IMP $(1)=1$ ] for carbon, oxygen, and iron if the detailed model choice $\operatorname{IMP}(3)=2$ is made. A rate equation approach is also possible through the use of the subroutine DYNAM [IMP(1) = 2] but is not currently implemented. A description of DYNAM is included in this section.

Diffusion is modeled by using formulae, by a numerical calculation using Pfirsch-Schliter diffusion coefficients, or by a combination of these two approaches. If the formulae are selected, the code arbitrarily sets the overall carbon, oxygen and/or "high-Z" spatial densities by the functions $F \emptyset T, F \emptyset X, F C T$, AND FCARX where:

$F \emptyset T$ gives the time evolution of the total number of oxygen ions in the discharge $[\mathrm{F} \emptyset \mathrm{T}(0)=1]$;

FCT does the same for carbon;

$F \emptyset X(X)$ gives the spatial distribution of the total number of oxygen iulls

$\left(2 \int_{0}^{1} \mathrm{dX} * \mathrm{X} * \mathrm{~F} \emptyset \mathrm{X}(\mathrm{X})=1\right)$; and

$\operatorname{FCARX}(\mathrm{X})$ does the same for carbon.

The detailed model options are as follows:

$\operatorname{IMP}(2)=1$, corona equ1librium tables and formulae for carbon and oxygen. A crude model is used for high-Z impurities.

$\operatorname{IMP}(2)=2$, corona equilibrium tables and formulae are used for carbon, oxygen, and high-Z (iron). 


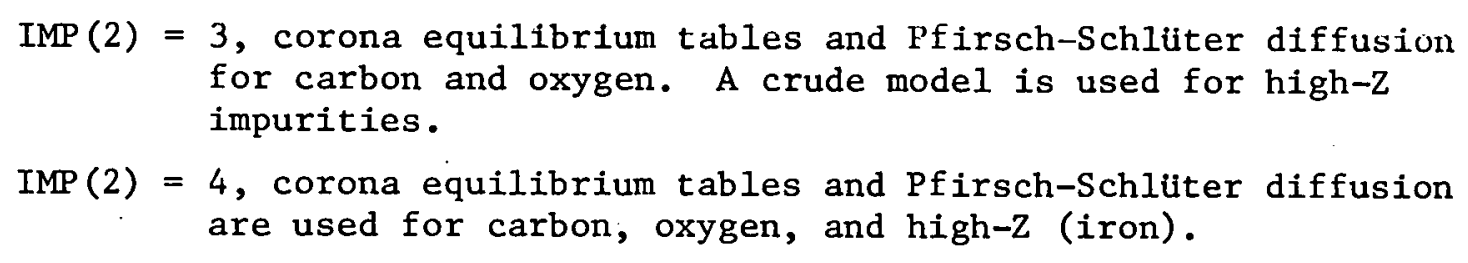

\subsection{BASIC INPUT/OUTPUT}

The basic input to this module consists of the total carbon, oxygen, and electron density profiles; the electron temperature profile; the profiles of the charge states of carbon; some timing and geometry values; edge density values for carbon and iron; and an array of switch values.

The hasic output consists of a set of new total carbon and oxygen density profiles, the effective charge of the plasma and the high-L impurity, power losses due to the impuritics, and synchrotronbremsstrahlung radiation. A plasma ion density profile is also generated.

\subsection{SUMMARY OF MODELS AVAILABLE}

The available models include use of corona equilibrium tables, solution of a diffusion equation using Pfirsch-Schluter diffusion coefficients, and a rate equation calculation which is not currently referenced by the program logic. (To employ the dynamic calculation, the cal1 to TABLE should be replaced by a call to DYNAM.)

\subsection{BRIEF DESCRIPTION OF EACH SUBROUTINE IN THE IMPURITY MODULE}

The subroutines described in this section will probably undergo more changes than those of any other module. Therefore, more attention has been given to defining some of the significant internal variables in the module. This should ease the task of making desired modificactons. 
SUBRØUTINE IMPRTY

Subroutine IMPRTY is the main driving program for the impurity module. Figure 7 shows which subroutines are called, their order of call, and the flow of the logic associated with the switches which determine the combination of models used. This subroutine does some of the basic bookkeeping and the calculations of the impurity profiles using the formulae.

Called from: MAIN

Subroutines called: FCT, FøT, FCARX, FØX, ZDIFFU, TABLE, HIGHZ, ZCALC, IMPRIN

Commons required: $\quad$ GE $\emptyset$ M, IMPURT, INDEX, I $\emptyset \mathrm{NS}$, RADL $\emptyset S$, SURF, TEMP, TIME, XPØRT, ZRAD

Variables required: See Table 11 and Appendix II.

Variables changed: See Table 11 and Appendix II.

Significant internal variables:

ICLK - a "clock" setting used to determine the frequency of printout of the density and effective charge profiles for the iron impurity. The density profiles of the various charge states of carbon and oxygen are also printed.

IST - a "timing" variable which keeps track of the number of times the impurity module is called, and is compared with the value of ICLK. When IST = ICLK, the impurity profiles are printed and the value of IST is reset to zero.

ICR - a "zero cross-over" switch used to initialize the values of total carbon and oxygen impurities on the first call to IMPRTY only

IICCT - a counter switch setting used to program logic control for the frequency with which low $Z$ diffusion is computed

IICC - counter variable for frequency of low-Z diffusion computation

IIFET - a counter switch setting, used for program logic control, which determines how often high-Z (Fe) impurity diffusion is computed

IIFE - counter variable for frequency of high-Z diffusion computation

FEO - central density of iron impurity, $\mathrm{cm}^{-3}$

HTC - elapsed time variable used for the simulation of the low $-Z$ impurity diffusion 
ORNL.OWG 76.2307
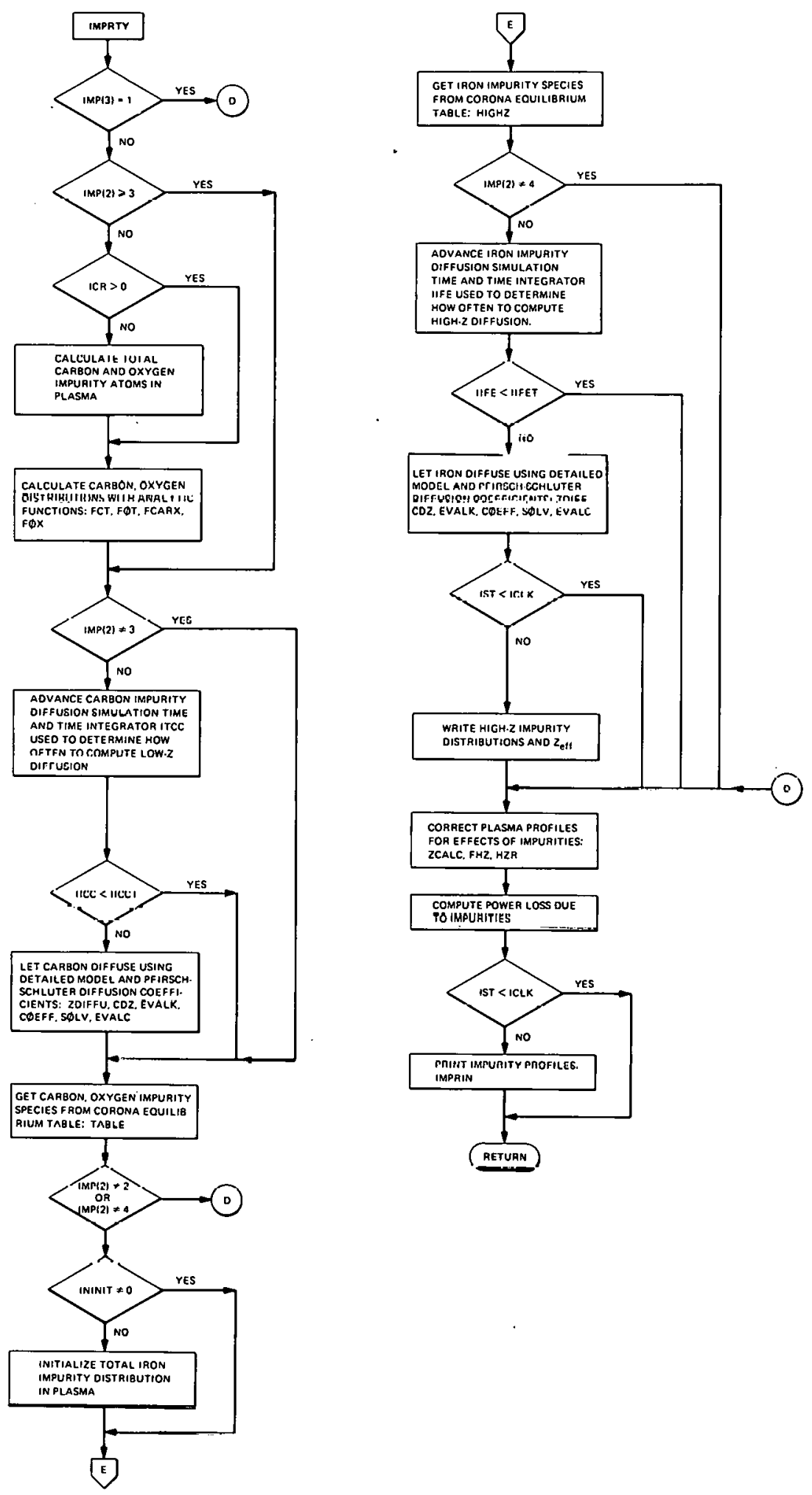

Fig. 7. Diagram of IMPRTY module and subroutines called. 
Table 11. List of labeled commons and elements in them which are referenced by subroutine IMPRTY

\begin{tabular}{|c|c|c|c|c|}
\hline \multirow{2}{*}{$C \emptyset \mathrm{MM} \varnothing \mathrm{N} \quad \mathrm{BL} \emptyset \mathrm{CK}$} & \multicolumn{2}{|c|}{ Input } & \multicolumn{2}{|c|}{$\emptyset$ utput } \\
\hline & Variables & Arrays & Variables & Arrays \\
\hline /GE $\mathrm{M}_{\mathrm{M}} /$ & $\mathrm{V} \emptyset \mathrm{L}$ & & & \\
\hline / IMPURT/ & PCIMP & CARB $\emptyset \mathrm{N}, \emptyset \mathrm{XYGEN}, \mathrm{CC}$ & & CARB $\emptyset \mathrm{N}, \emptyset \mathrm{XYGEN}$ \\
\hline /INDEX/ & $\mathrm{N}, \mathrm{NM1}$ & IMP & & \\
\hline$/ \mathrm{I} \emptyset \mathrm{NS} /$ & & DEN & & \\
\hline$/ \mathrm{RADL} \emptyset \mathrm{S} /$ & & & & $\mathrm{Al}$ \\
\hline /SURF/ & CARN, FENS & & $\operatorname{ISR} \emptyset \mathrm{N}$ & \\
\hline /TEMP/ & & $\mathrm{TE}$ & & \\
\hline /TIME/ & HT, TIM, TMLAX & & & \\
\hline /ZRAD/ & & & & $\mathrm{QRD}$ \\
\hline /XPøRT/ & & & & D1 \\
\hline /ZRAD/ & & & & HIZ \\
\hline
\end{tabular}


HTFE - elapsed time variable used for the high-Z impurity diffusion simulation

ININIT - a "zero cross-over" switch used to initialize the total iron impurity profile and high-Z impurity ( $\mathrm{Fe}$ ) value of $z_{\text {eff }}$ 
FUNCTI $\emptyset N$ FCT $(T)$

Function FCT allows for programming the carbon impurity content of the plasma discharge throughout the simulation time. This subroutine currently sets the value of FCT to unity for all times.

Called from: IMPRTY

Variable required:

$\mathrm{T}$ - fraction of elapsed simulation time

Variable changed:

FCT - value of factor expressing time dependence of total carbon content of the plasma 
FUNCTI $\emptyset \mathrm{N} \quad F \emptyset T(T)$

Function FØT allows for programming the oxygen impurity content of the plasma discharge throughout the simulation. The value of this function is currently set to unity for all times.

Called from: IMPRTY

Variable required:

$$
\mathrm{T} \text { - fraction of elapsed simulation time }
$$

Variable changed:

$F \emptyset \mathrm{T}$ - value of factor expressing time dependence of total oxygen content of the plasma. 
FUNCTI $\emptyset \mathrm{N}$ FCARX(X)

Function FCARX is used to program the profile of the total combined carbon species present at each point $X$ in the plasma. The value of this function is currently set, to unity for all radial values.

Called from: IMPRTY

Variable required:

$\mathrm{X}$ - radial position in the plasma, scaled to the torus minor radius

Variable changed:

FCARX - value of total carbon profile form factor at position $X$ 
FUNCTI $\emptyset \mathrm{N} F \emptyset \mathrm{X}(\mathrm{X})$

Function $F \emptyset X$ is used to program the profile of the total combined oxygen species present at each point $X$ in the plasma. The value of this function is currently set to unity for all radial values.

Called from: IMPRTY

Variable required:

$X$ - radial position in the plasma, scaled to the torus minor radius

Variable changed:

$F \emptyset X$ - value of total. oxygen profile form factor at posilion $X$ 
SUBRØUTINE TABLE (TE,N)

Subroutine TABLE contains tables of values of the fractions of total density of the elements carbon and oxygen in each ionization state as a function of temperature. These fractions were computed on the basis of a corona equilibrium model of electron impact ionization. The fractions are given for temperature intervals of $10 \mathrm{eV}$ and a linear interpolation is used to compute the fractions for an arbitrary value of the temperature. Called from: IMPRTY

Commons required: IMPURT

Variables required: $\mathrm{TE}, \mathrm{N}$

Arrays changed:

$$
\begin{aligned}
\mathrm{CC}(\mathrm{K}, \mathrm{I}) \text { - } & \text { density of the }(\mathrm{K}-1) \text { th charge state of carbon at the } \\
& \text { i-th grid point } \\
\mathrm{C} \emptyset(\mathrm{K}, \mathrm{T}) \text { - } & \text { density of the }(\mathrm{K}-1) \text { th charge state of oxygen at the } \\
& \text { i-th grid point }
\end{aligned}
$$

Significant internal arrays:

$\mathrm{Cl}$ - table of fractions of total density of carbon in the ground state as a function of temperature

$\mathrm{C} 2$ - same as $\mathrm{C} 1$, except for first ionization state

C3 - same as C1, except for second ionization state

$\cdots \quad \cdots$

C7 - same as $\mathrm{Cl}$, except for sixth ionization state

$\emptyset 1$ - table of fractions of total density of oxygen in the ground state as a function of temperature

$\emptyset 2$ - same as $\emptyset 1$, except for first ionization state

- .

$\emptyset 9$ - same as $\emptyset 1$, except for eighth ionization state 
SUBR $\emptyset U T I N E ~ Z C A L C$ (TE, D1, DEN, ZFE, ZIR $\emptyset N$, IIR $\emptyset N)$

Subroutine ZCALC computes the following profiles:

D1

$$
z_{\text {eff }} \equiv \sum_{k}\left(n_{k} z_{k}^{2}\right) / n_{e}
$$

DENP

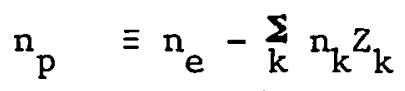

ZBR

$$
[Z] \equiv\left[\sum_{k}\left(n_{k} z_{k}^{2}\right) / A_{k}\right] / n_{e}
$$

QRD power loss due to line radiation from low-Z elements, $\mathrm{eV} / \mathrm{msec} / \mathrm{cm}^{3}$

HIZ

$$
\begin{aligned}
& \text { power loss due to line radiation from high }-\mathrm{Z} \text { elements, } \\
& \mathrm{eV} / \mathrm{msec} / \mathrm{cm}^{3}
\end{aligned}
$$

These profiles can be computed using a detailed model [ IMP(3) = 1] or an analytic model [ IMP(3) $=2]$.

In the detailed model calculation, the profiles are calculated by considering the impurity species

i) carbon - charge states 1 through 6 ,

ii) oxygen - charge states 1 through 8 , and

i i i) "high-7." - stripped according to the formula $Z=\sqrt{\mathrm{TE} / 13.6}$ * ZIMP, where ZIMP is the atomic charge number.

The auxiliary values

$$
\begin{aligned}
& X \emptyset I={ }_{J}^{\Sigma} C \emptyset(J, I) * Z_{J} / 16 \\
& \mathrm{XC1}=\mathrm{J}_{\mathrm{J}} \mathrm{CC}(\mathrm{J}, \mathrm{I}) * \mathrm{Z}_{\mathrm{J}} / 12 \\
& X C \phi I=\sum_{J} C \emptyset(J, I) * z_{J} / D E N(I) \\
& \mathrm{XCCl}=\sum_{\mathrm{J}}^{\mathrm{S}} \mathrm{CC}(\mathrm{J}, \mathrm{I}) * \mathrm{z}_{\mathrm{J}} / \operatorname{DEN}(\mathrm{I}) \\
& x C \phi 2=\bar{J}_{\mathrm{J}} \mathrm{C} \phi(\mathrm{J}, \mathrm{I}) * \mathrm{z}_{\mathrm{J}}{ }^{2} / \operatorname{DEN}(\mathrm{I})
\end{aligned}
$$

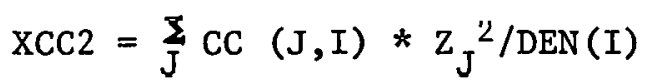

are defined for each grid point $I$. The charge state for the "high-Z" element is modeled by assuming the charge to be

$$
\mathrm{ZI}= \begin{cases}0 & \mathrm{TE}<13.6 \\ \sqrt{\mathrm{TE} / 13.6} & 13.6<\mathrm{TE}<13.6 *(\mathrm{ZIMP})^{2} \\ \mathrm{ZIMP} & \mathrm{TE}>13.6 *(\mathrm{ZIMP})^{2}\end{cases}
$$


The "high-Z" impurity density is assumed to be

$$
\operatorname{DENZ}=\operatorname{PCIMP} * .01 * \operatorname{DEN}(1) * \operatorname{DHZ}(\mathrm{X}) \text {, }
$$

where DHZ is a chosen function of $X(=r / a)$.

Thus, for $\mathrm{Z}_{\text {eff }}$

$$
\mathrm{D} 1=\text { 1. }+\mathrm{XC} \emptyset 2+\mathrm{XCC} 2+\operatorname{DENZ} *(\mathrm{ZI})^{2} / \mathrm{DEN}(\mathrm{I}) \text {, }
$$

for $n_{p}$

$$
\mathrm{DENP}=1 .-\mathrm{XC} 1-\mathrm{XCC} 1-\mathrm{DENZ} * \mathrm{ZI} / \mathrm{DEN}(\mathrm{I}) \text {, }
$$

for $[z]$

$$
\mathrm{ZBR}=1 .+\mathrm{X} \emptyset 2+\mathrm{XC} 2
$$

(the "high-Z" impurity is assumed to have infinite mass), for low-Z radiation power loss

$$
\begin{aligned}
\mathrm{QRD}= & 9.18(10)^{-10} * \sqrt{13.6 / \mathrm{TE}} * \mathrm{~N}_{\mathrm{e}} *\left[\mathrm{~N}_{2}^{0} \mathrm{e}^{-16 / \mathrm{TE}}\right. \\
& +\mathrm{N}_{3}^{O} \mathrm{e}^{-18 / \mathrm{TE}}+\mathrm{N}_{4}^{0} \mathrm{e}^{-19 / \mathrm{TE}}+\mathrm{N}_{5}^{0} \mathrm{e}^{-20 / \mathrm{TE}} \\
& +\mathrm{N}_{6}^{O} \mathrm{e}^{-12 / \mathrm{TE}}+2 \mathrm{~N}_{7}^{0} \mathrm{e}^{-575 / \mathrm{TE}}+2 \mathrm{~N}_{8}^{0} \mathrm{e}^{-655 / \mathrm{TE}} \\
& \left.+\mathrm{N}_{8}^{0} \mathrm{e}^{-83 / \mathrm{TE}}\right] \\
& +2.04(10)^{-10} \sqrt{1.3 .6 / \mathrm{TF}} * \mathrm{~N}_{\mathrm{e}} *\left[\mathrm{~N}_{3}^{\mathrm{C}} \mathrm{e}^{-12 / \mathrm{TE}}\right. \\
& +\mathrm{N}_{4}^{\mathrm{C}} \mathrm{e}^{-8 / \mathrm{TE}}+\mathrm{N}_{5}^{\mathrm{C}} \mathrm{e}^{-308 / \mathrm{TE}}+\mathrm{N}_{6}^{\mathrm{C}} \mathrm{e}^{-370 / \mathrm{TE}} \\
& \left.+\mathrm{N}_{5}^{\mathrm{C}} \mathrm{e}^{-40 / \mathrm{TE}}\right],
\end{aligned}
$$

and for high-Z radiation power loss

$$
\mathrm{HIZ}=\left\{.4+4.6 * \operatorname{EXP}\left[-10^{-6} *(\mathrm{TE})^{2}\right]\right\} * \mathrm{DEN} * \mathrm{DENZ} * 4.17(10)^{-11}
$$


For the analytic model, the profiles are given by simple analytic expressions :

$\mathrm{z}_{\mathrm{p}}$

[Z]

low-Z radiation power loss

high-Z radiation power loss
$\mathrm{D} 1=\mathrm{ZEFF}$

$\mathrm{DENP}=\mathrm{DEN}$

$\mathrm{ZBR}=1$

$\mathrm{QRD}=0.0$

$\mathrm{HIZ}=4.17(10)^{18} * \mathrm{PHZ} * \mathrm{HZR}(\mathrm{X}) / \mathrm{V} \emptyset \mathrm{L}$,

where $\mathrm{PHZ}$ is the assumed power loss in kilowatts, specified in a DATA statement, and HZR is a pieselected function of $X(=r / a)$. [Note that

$$
\left.2 \int_{0}^{1} \mathrm{dX} * \operatorname{HZR}(\mathrm{X}) * \mathrm{X}=1 .\right]
$$

7. ${ }_{\text {eff }}$ is represented by $\mathrm{ZEFF}$, an input parameter read in at the beginning of the simulation.

Called from: IMPRTY

Common required: IMPURT

Variables and arrays required (see also Table 12):

$\mathrm{TE}$ - electron temperature profile, eV

DI - protile of effective clialge $Z_{\text {eff }}$

$\mathrm{N} \quad-$ number of radial polnts in the spatial grid

DEN - electron density profile, $\mathrm{cm}^{-3}$

IMP(3) - switch for type of model $=1$, detailed calculation

$=2$, analytic model formulae

ZFE - effective charge profile of the iron impurtty

ZIR $\oint_{N}$ - density prolile of the 1ron impurity, $\mathrm{cm}^{3}$

IIR $\emptyset N$. switch uood to determine whether high-Z effects are to be modeled using iron or a general "high- $Z$ " impurity species

Arrays changed:

DENP - plasma proton density profile, $\mathrm{cm}^{-3}$

ZBR - the profile of [Z]

D1 - effective charge $Z_{\text {eff }}$ profile

QRD - low-Z impurity radiation contribution to the power loss, $\mathrm{eV} /\left(\mathrm{msec} \cdot \mathrm{cm}^{3}\right)$

HIZ - hi.gh-Z impurity radiation contribution to the power loss, $\mathrm{eV} /\left(\mathrm{msec} \cdot \mathrm{cm}^{3}\right)$ 
Table 12. List of labeled commons and elements in them which are referenced by subroutine ZCALC

\begin{tabular}{|c|c|c|c|c|}
\hline \multirow{2}{*}{$\mathrm{C} \emptyset \mathrm{MM} \emptyset \mathrm{N} \quad \mathrm{BL} \emptyset \mathrm{CK}$} & \multicolumn{2}{|c|}{ Input } & \multicolumn{2}{|c|}{ Output } \\
\hline & Variables & Arrays & Variables & Arrays \\
\hline$/ \mathrm{GE} \emptyset \mathrm{M}$ & $\mathrm{v} \emptyset \mathrm{L}$ & & & \\
\hline /INDEX/ & $\mathrm{N}$ & IMP & & \\
\hline /IMPURT/ & PCIMP, ZEFF, ZIMP & $\mathrm{c} \emptyset, \mathrm{cc}$ & & DENP, ZBR \\
\hline /ZRAD/ & & $\mathrm{DHI}, \mathrm{ZHI}$ & & $\mathrm{QRD}, \mathrm{HIZ}$ \\
\hline
\end{tabular}


SUBRøUTINE ZDIFFU (VAR, SIV, HT)

Subroutine ZDIFFU advances the impurity atom population profile VAR(I) having charge distribution ZIV(I). ZDIFFU uses subroutine CDZ to compute the needed diffusion coefficients. The following diffusion equation was derived by Rutherford ${ }^{l}$ for the Pfirsch-Schliter regime.

$$
\begin{aligned}
& \frac{\partial}{\partial t} \operatorname{VAR}(r, t)=\frac{1}{r} \frac{\partial}{\partial r}\left[r * \operatorname{TDZZ} * \frac{\partial}{\partial r} \operatorname{VAR}(r, t)\right] \\
& -\frac{1}{r} \frac{\partial}{\partial r}\left\{r * \operatorname{VAR}(r, t) * Z_{\mathrm{N}} \operatorname{VV}(r, t) * \operatorname{TD} Z Z *\left(\mathrm{~L}_{\mathrm{T}}-1\right] \mathrm{C}_{1}\left(1-\frac{1}{\operatorname{ZIV}(r, t)}\right)\right. \\
& \left.\left.\left.-\mathrm{C}_{2}\right]+\mathrm{C}_{1} \mathrm{~L}_{\mathrm{P}}=1\right)\right\}
\end{aligned}
$$

where

$$
\begin{aligned}
& \mathrm{L}_{\mathrm{T}}^{-1}=\frac{\mathrm{dT}}{\mathrm{dr}} \frac{1}{\mathrm{~T}_{i}}, \mathrm{~L}_{\mathrm{P}}^{-1}=\frac{\mathrm{dN}}{\mathrm{dr}} \frac{1}{\mathrm{~N}_{\mathrm{p}}}, \\
& \mathrm{C}_{1}=0.47+\frac{0.35}{0.66+\alpha}, \\
& \mathrm{C}_{2}=0.30+\frac{0.41}{0.58+\alpha}, \\
& \alpha=\operatorname{VAR}^{*}(\mathrm{ZIV})^{2} / \mathrm{N}_{\mathrm{p}},
\end{aligned}
$$

TDZZ is computed in CDZ (Note: it is scaled so that $0 \leqslant r \leqslant 1$ ), and

$$
\text { ZGKAU }=a L_{T}+\left[\left(1-\frac{1}{\operatorname{ZIV}(r, t)}\right)-\frac{C_{3}}{C_{1}}\right]+a L_{p}^{-1}
$$

is also computed in $\mathrm{CDZ}$.

The numerical method for solving the diffusion equation is predictorcorrector and is contained in the subroutines EVALK, C $\emptyset \mathrm{EFF}$, and S $\emptyset \mathrm{LVE}$. These routines solve the general one-dimensional, time-dependent equation:

$$
\frac{\partial u}{\partial t}=\frac{1}{r} \frac{\partial}{\partial r}\left[r k(u) \frac{\partial u}{\partial r}\right]-\frac{1}{r} \cdot \frac{\partial}{\partial r}[r f]
$$

where $\mathrm{f}$ is a given inhomogeneous term. 
Subroutine IMPRTY prepares the diffusion step by loading the appropriate profiles into the arrays VAR and ZIV and be selecting the value of the time step HT to be advanced (this may be different from the time step used for the plasma evolution in the main part of the simulation code). The differencing scheme in the immediately preceding equation is based on the Crank-Nicholson representation

where

$$
\begin{aligned}
& \frac{u_{i}^{N}-u_{i}}{\Delta t}=\frac{1}{r} \frac{1}{2}\left(\frac{\left[r k \frac{\partial u}{\partial r}\right]_{i+1 / 2}^{N}-\left[r k \frac{\partial u}{\partial r}\right]_{i-1 / 2}^{N}}{\Delta r}\right. \\
& \left.\quad+\frac{\left[r k \frac{\partial u}{\partial r}\right]_{i+1 / 2}-\left[r k \frac{\partial u}{\partial r}\right]_{i-1 / 2}}{\Delta r}\right)-\frac{1}{r} \frac{\tilde{f}_{i+i}-\tilde{f}_{i-1}}{2 \Delta r}
\end{aligned}
$$

$$
\left.\frac{\partial u}{\partial r}\right|_{i+1 / 2}=\frac{u_{i+1}-u_{i}}{\Delta r},\left.\frac{\partial u}{\partial r}\right|_{i+1 / 2}=\frac{u_{i}-u_{i-1}}{\Delta r} \text {, and } \tilde{f}=r f \text {. }
$$

The steps used in the predictor-corrector cycle are:

1) predict - compute $u_{i}^{t}+\frac{\Delta t}{2}$ using $k\left[u_{i}(t)\right]$

2) compute $u_{i}^{t+\Delta t}$ using $k\left[u_{1}\left(t+\frac{\Delta t}{2}\right)\right]$

3) correct - compute $u_{i}^{t}+\frac{\Delta t}{2}$ using

$$
u_{i}^{t}+\frac{\Delta t}{2}=\frac{1}{2}\left[u_{i}^{o l d}+u_{i}^{t+\Delta t}\right]
$$

and compare this with the prediction. If the prediction is within a specified tolcrance, continue witl the simulation. Otherwise

4) iterate the eequencc

$$
u_{i}^{(m)}\left(t+\frac{\Delta t}{2}\right)=\frac{1}{2}\left[u_{i}^{o l d}(t)+u_{i}^{(m)}(t+\Delta t)\right] \text {. }
$$

Each new $u_{i}^{(m)}\left(t+\frac{\Delta t}{2}\right)$ is gotten from a $u_{i}^{(m)}(t+\Delta t)$ in Step 2 using $k\left[u_{i}^{m-1}\left(t+\frac{\Delta t}{2}\right)\right]$ until the tolerance criterion is satisfied. 
When the iteration has converged (or after one correction step in the current code), the array VAR is set equal to the array $\mathrm{Xl}$, the value of the dependent variable at the new time.

Called from: IMPRTY

Subroutines called: EVALC, EVALK, CøEFF, SøLVE

Commons required: GE $\emptyset \mathrm{M}$, INDEX, ZDFPS

Variables required:

$\operatorname{VAR}(101)$ - impurity species density proftle, $\mathrm{cm}^{-3}$

Z.TV(101) - effective charge profile of impurity spectes

HT - time increment for the impurity diffusion, msec

From labeled common blocks (see Appendix II for definltions)

/GE $\emptyset \mathrm{M} /$ - AM

/INDEX/ - N, NMI

/ZDFPS/ - TDZZ(101), ZGRAD(101)

Variables changed: VAR(101)

Significant internal variables:

$\mathrm{Xl}(101)$ - a temporary (local) array for the 1mpurity species density profile

ICY - subscript variable used to determine the number of corrector iteration cycles for the predictor-corrector method of solution 
SUBRфUTINE EVALC (ZK,ZF, V,Z,AM,N)

Subroutine EVALC computes the profiles of the diffusion coefficient $k(u)$ and the inhomogeneous term $f$ of the diffusion equation referred to in the description of subroutine ZDIFFU (p. 124). These profiles must be recomputed for each new. time in the diffusion process.

Called from: $\quad$ ZDIFFU

Subroutines called: $\mathrm{CDZ}$

Common required: $\quad$ ZDFPS

Variables required:

$\mathrm{V}(101)$ - total density of an impurity species of a given atomic number, $\mathrm{cm}^{-3}$

$Z$ (101) - effective charge profile of the given impurity species

$\mathrm{AM} \quad-$ torus minor radius, $\mathrm{cm}$

N - number of radial grid points

Variables changed:

$$
\begin{aligned}
& \text { ZK (101) - profile of } k(u) \\
& Z F(101) \text { - profile of } f
\end{aligned}
$$


SUBRØUTINE CDZ (VAR, ZIV)

Subroutine $\mathrm{CDZ}$ computes the impurity ion diffusion coefficients for the Pfirsch-Schlüter regime. They are given by the expressions

$$
\operatorname{TDZZ}(I)=\nu_{i} \rho^{2}\left[\frac{r}{R_{0}} \frac{B_{T}}{B}\right]^{2} C_{1}(10)^{-3}
$$

and

$$
\operatorname{ZGRAD}(I)=\frac{\mathrm{a}_{\mathrm{m}}}{\mathrm{N}_{\mathrm{p}}} \frac{\mathrm{dN}_{\mathrm{p}}}{\mathrm{dx}}+\frac{\mathrm{a}_{\mathrm{m}}}{\mathrm{T}_{\perp}} \frac{\mathrm{dT}_{\mathrm{i}}}{\mathrm{dr}}\left\{\left[1 .-\frac{1}{\operatorname{ZIV}(I)}\right]-\frac{\mathrm{c}_{2}}{\mathrm{c}_{1}}\right\}
$$

where

$$
\begin{aligned}
& v_{i}=\frac{1.13(10)^{-6}}{\sqrt{M}} \mathrm{~N}_{\mathrm{e}}\left[1 .-\frac{\ln \left(\mathrm{N}_{\mathrm{e}} / \mathrm{T}_{\mathrm{e}}\right)}{17.25}\right] \mathrm{Z}_{\text {eff }} \mathrm{T}_{\mathrm{e}}^{-3 / 2}, \\
& \rho^{2}=2.09(10)^{4} \mathrm{MT}_{i} / \mathrm{B}_{\mathrm{T}}^{2}, \\
& c_{1}=\frac{0.47+0.35}{0.66+\alpha}, \quad c_{2}=\frac{0.3+0.41}{0.58+\alpha},
\end{aligned}
$$

and

$$
a=\operatorname{VAR}(I) * \operatorname{ZIV}(I) * Z I V(I) / N_{\mathrm{e}}
$$

Subroutine CDZ prints the arrays VAR, ZIV, TDZZ, and ZGRAD the first time it is called.

\section{Called from: EVALC}

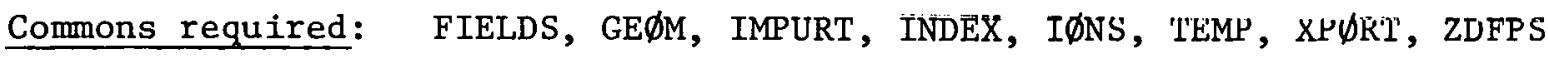
Variables required:

$\operatorname{VAR}(101)$ - total density of an impurity species of a given atomic number

ZIV(101) - effective charge profile of the given impurity species

From labeled common blocks (see Appendix II for definitions)

$$
\begin{aligned}
& \text { /FIELDS/ - B(101), BT } \\
& \text { /GE } \emptyset \text { / - AM, HR, R } ~ \\
& \text { /IMPURT/ - DENP(101) } \\
& \text { /INDEX/ - N } \\
& \text { /IONS/ - DEN(101), AMU } \\
& \text { /TEMP/ - TE(101), TI(101) } \\
& \text { /XPøRT/ - DI(101) }
\end{aligned}
$$


Variables changed:

TDZZ(101) - impurity species diffusion coefficient

ZGRAD(101) - a collection of ion temperature and density gradient terms

Significant internal variables:

ZNUI - ion collision frequency

RH $\varnothing 2$ - ion gyroradius 
SUBRØUTINE EVALK (ZK, N, ZKP, ZKM)

Subroutine EVALK calculates the arrays

$\left.\begin{array}{l}\operatorname{ZKP}(I)=.5^{*}(\mathrm{ZK}(I)+\mathrm{ZK}(I+1)) \\ \operatorname{ZKM}(I)=.5^{*}(\mathrm{ZK}(I)+\mathrm{ZK}(I-1))\end{array}\right\} \quad I=2, \ldots, N-1$

for the function $k(u)$ in the predictor-corrector algorithm for obtaining the solution to

$$
\frac{\partial u}{\partial t}=\frac{1}{r} \frac{\partial}{\partial r}\left[r k(u) \frac{\partial u}{\partial r}\right]-\frac{1}{r} \frac{\partial}{\partial r}[r f],
$$

set up in subroutine ZDIFHU.

Galled from:

7.TTFFII

Variables required:

$$
\begin{aligned}
\mathrm{ZK}(\mathrm{I})- & \mathrm{TDZZ}(\mathrm{I}) / \mathrm{AM} / \mathrm{AM}=\mathrm{D}(\mathrm{r}) / \mathrm{a}_{\mathrm{m}}^{2} \text {, the impurity particle diffusion } \\
& \text { coefficient scaled by the torus minor radius } \\
\mathrm{N} \quad- & \text { the number of radial nodes }
\end{aligned}
$$

Variables changed:

$\mathrm{ZKP}$ - forward difference of $\mathrm{ZK}$

$\mathrm{ZKM}$ - backward difference of $\mathrm{ZK}$ 
SUBR $\emptyset U T I N E ~ C \emptyset E F F(Z K P, Z K M, A, B, C, D, E, F, S 1, N, V, Z F)$

Subroutine CØEFF computes the coefficients of the values of the impurity atom density $u$ at the new time step in the set of linear difference equations which are obtained from the Crank-Nicholson representation of

$$
\frac{\partial u}{\partial t}=\frac{1}{r} \frac{\partial}{\partial r}\left[r k(u) \frac{\partial}{\partial r}\right]-\frac{1}{r} \frac{\partial}{\partial r}[r g]
$$

where $g(r)$ is a given inhomogeneous term. The Crank-Nicholson representation is

$$
\frac{u_{i}^{N}-u_{i}}{\Delta t}=\frac{1}{r} \frac{1}{2}\left\{\delta^{N}+\delta\right\}-\frac{1}{r} \frac{f_{i+1}-f_{i-1}}{2 \Delta r}
$$

where

$$
\begin{aligned}
& \delta^{N}=\frac{1}{\Delta r}\left\{\left[\operatorname{rk}(u) \frac{\partial u}{\partial r}\right]_{i+1 / 2}^{N}-\left[r k(u) \frac{\partial u}{\partial r}\right]_{i-1 / 2}^{N}\right\}, \\
& \delta=\frac{1}{\Delta r}\left\{\left[\operatorname{rk}(u) \frac{\partial u}{\partial r}\right]_{i+1 / 2}-\left[\operatorname{rk}(u) \frac{\partial u}{\partial r}\right]_{i-1 / 2}\right\}, \\
& \left.\frac{\partial u}{\partial r}\right|_{i+1 / 2}=\frac{u_{i+1}-u_{i}}{r},\left.\quad \frac{\partial u}{\partial r}\right|_{i-1 / 2}=\frac{u_{i}-u_{i-1}}{\Delta r}, f=r g
\end{aligned}
$$

and

$$
\left[r k(u) \frac{\partial u}{\partial r}\right]_{i+1 / 2}=\frac{1}{\Delta r}\left(r+\frac{\Delta r}{2}\right) \frac{1}{2}\left(k_{i}+k_{i+1}\right)\left(u_{i+1}-u_{i}\right) .
$$

The Crank-Nicholson representation can be rearranged to the form

$$
-A_{i} u_{i+1}^{N}+B_{i} u_{i}^{N}-C_{i} u_{i-1}^{N}=D_{i}
$$

Subroutine C $\varnothing \mathrm{EFF}$ also evaluates the arrays $E$ and $F$ which are used in the Gauss elimination procedure to solve for $u$ : 


$$
E_{i}=\frac{A_{i}}{B_{i}-C_{i} E_{i-1}} \quad \text { and } \quad F_{i}=\frac{D_{i}+C_{i} F_{i-1}}{B_{i}-C_{i} E_{i-1}}
$$

\section{Called from: $\quad$ ZDIFFU}

Variables required:

$$
\begin{aligned}
& \text { ZKP(101) - forward difference of the diffusion coefficient, } k(u) \\
& \mathrm{ZKM}(101) \text { - backward difference of the diffusion coefficient, } k(u) \\
& \text { S1 - reciprocal of the time step for the evolution of the } \\
& \mathrm{N} \quad-\text { the number of spallal nodes. } \\
& \text { U(101) - impurity species density prufile at the current } \\
& \text { value of elapsed time, } \mathrm{cm}^{-3} \\
& \text { ZF(101) - profile of function appearing in the Inhomogeneous } \\
& \text { term of the diffusion equation }
\end{aligned}
$$

Variables changed:

$$
\left.\left.\begin{array}{l}
\text { A(101) } \\
B(101) \\
C(101)
\end{array}\right\} \begin{array}{l}
\text { coefficients appearing in the set of linear equations } \\
\text { for the value of the density profile at the new time. } \\
\text { The set of equations arises from the Crank-Nicholson } \\
\text { finite differencing scheme for solving the diffusion } \\
\text { equation. } \\
\text { D(101) - inhomogeneous term in the set of linear equations } \\
\text { obtained from the finite differencing scheme } \\
\text { F(101) }
\end{array}\right\} \begin{aligned}
& \text { - arrays used in the Gaussian elimination procedure } \\
& \text { to solve for the impurity species density profile at the } \\
& \text { new time step }
\end{aligned}
$$


SUBR $\emptyset \mathrm{UTINE}$ S $\emptyset \mathrm{LVE}(\mathrm{U}, E, F, N)$

Subroutine SøLVE generates the solution of the impurity atom diffusion equation for the new time value. It actually performs the Gaussian elimination using the $E$ and $F$ arrays generated by subroutine CØEFF. The routine sweeps

$$
u_{i}=E_{i} u_{i+1}+F_{i}
$$

from $r=a^{-}(i=N-1)$ to $r=0(i=1)$.

Called from: $\quad$ ZDIFFU

Variables required.:

$$
\left.\begin{array}{l}
\mathrm{U}(101) \quad \begin{array}{r}
\text { - impurity species density profile at the old time value } \\
\text { (upon entry into this subroutine) }
\end{array} \\
\mathrm{E}(101) \\
\mathrm{F}(101)
\end{array}\right\} \begin{aligned}
& \text { - } \begin{array}{l}
\text { coefficients computed in setting up the Gaussian } \\
\text { elimination procedure }
\end{array} \\
& \mathrm{N}
\end{aligned}
$$

Variables changed: $\mathrm{U}(101)$ 
FUNCTI $\emptyset \mathrm{N} \operatorname{HZR}(I, \mathrm{X})$

Function HZR returns a value for the high-Z impurity radial profile form factor using the formula

$$
\mathrm{HZR}=(I+1) *(1 .-X * X) * * I
$$

Called from:

ZCALC

Variables required:

I - value of the exponent in the form factor formula

$\mathrm{X}$ - radial variable, normalized to the torus minor radius

Variables changed:

HZR - value of the form factor at $X$

Note: The user is free to choose any formula that seems appropriate for this form factor. 
SUBR ØUTINE HIGHZ (TE, ZIR $\emptyset \mathrm{N}, \mathrm{ZFE})$

Subroutine HIGHZ contains tables of fractional values of total iron density in each ionization state as a function of temperature. These fractions were computed on the basis of a corona equilibrium model of electron impact ionization. The fractions are given for temperature intervals of $0.1 \mathrm{keV}$ starting at $5.0 \mathrm{keV}$ at the lowest energy considered and extending to $9.0 \mathrm{keV}$. Three separate cases are treated:

i) If $\mathrm{T}_{\mathrm{e}}<\mathrm{TS}(=5.0 \mathrm{keV})$, all the iron ions are in the lowest charge state (in the current version of this subroutine, the lowest charge state is $3+$ ).

ii) If $\mathrm{TS} \leqslant \mathrm{T} \leqslant \mathrm{TT} \emptyset \mathrm{P}(=9.0 \mathrm{keV})$, a linear interpolation is used to compute the fractions for a given value of $\mathrm{T}_{\mathrm{e}}$.

iii) If $T T \phi P<T_{e}$, all the iron ions are in the highest charge state (in current version of the subroutine, the highest charge state is $26+$ ).

A printout of the TE array and selected parts of the RFE array is given the first time subroutine HIGHZ is called.

Called from: IMPRTY

Common required: INDEX

Variables required:

TE(101) - electron temperature profile, eV

ZIRøN(101) - ion impurity (all charge states included) density profile

From labeled common block (see Appendix II for definitions)

/INDEX/ - N

Varialiles changcd:

ZFE(101) - effective charge profile of iron impurities

Significant internal variables:

$\operatorname{AFE}(45,30)$ - array of exponents for the fraction of iron atoms in each charge state $(K+3)$ at a given temperature (index L)

$\operatorname{RFE}(101,30)$ - profiles of fractions of iron impurities in a particular charge state $\mathrm{K}+3$ 
SUBRØUTINE DYNAM (TE, DEN, DT, N)

Subroutine DYNAM calculates impurity stripping dynamically using electron impact ionization and recombination coefficients, determined analytically in a rate equation, to determine the densities of the various charge states of carbon and oxygen as a function of temperature. The ionization and recombination coefficients are supplied by subroutines SVCARB and SV $\varnothing X Y G$.

The plasma simulation time interval is subdivided into ten intervals. The rate equation has the general form

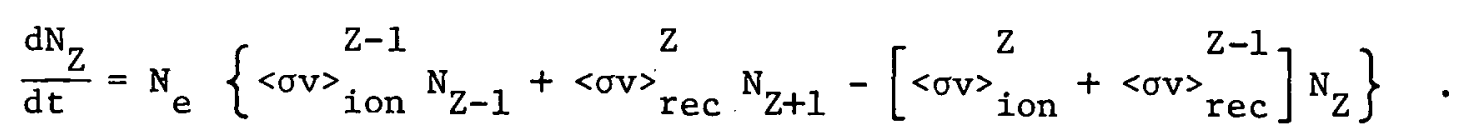

This equation is integrated over the sub-intervals of time using the general expression

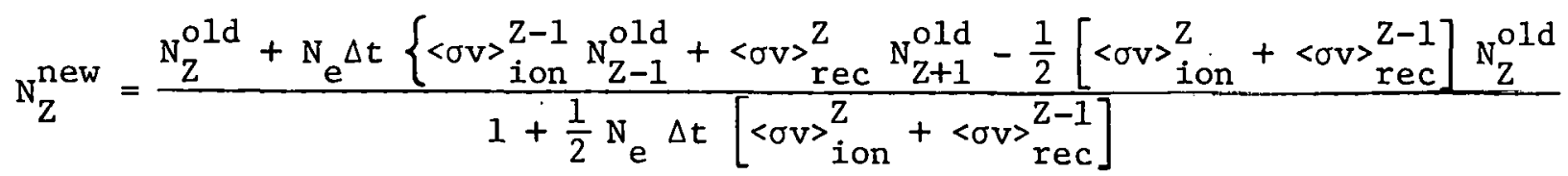
to generate the density of each charge state at each step of the time integration.

The general expression above is modilied approprlately for the neutral and completely ionized species. The neutral state cannot be fed from ion species below it by ionization since it is the lowest state and cannot feed a lower state by recombination. The completely ionized state cannot be fed from above by recombination nor can it feed anything higher by ionization. The integrations for these two states are thus done explicitly by themselves.

The total densties of carbon and oxygen impurities are computed from the densities of the various charge states of each element.

Called from:

Subroutines called:

Commons required:

Variables and arrays required:
Not currently used. SVCARB, SV $\emptyset \mathrm{XYG}$

IMPURT

TE, DEN, DT, N 
Arrays changed:

$$
\begin{aligned}
& \mathrm{CC}(\mathrm{K}, \mathrm{I}) \text { - density of the }(\mathrm{K}-\mathrm{I}) \text { th charge state of carbon at the } \\
& C \emptyset(K, I) \text { - density of the (K-1)th charge state of oxygen at the }
\end{aligned}
$$




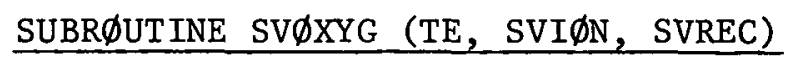

Subroutine SV $\varnothing \mathrm{XYG}$ generates ionization $\langle\sigma v\rangle_{\text {ion }}^{Z}$ and recombination $<\sigma v{ }_{\text {rec }}^{Z}$ coefficients for the different charge states of oxygen. These coefficients are required by subroutine DYNAM for calculating the densities of the various oxygen impurity charge states. The coefficients are generated using the analytic expressions ${ }^{2}$

$$
<\sigma v\rangle_{\text {ion }}^{L}=\left\{\begin{array}{lll}
A_{Z}^{\text {ion }} & e^{-\beta} \frac{\sqrt{\beta}}{\beta+x_{Z}^{i o n}}, & I_{Z} \leqslant 100 T_{e} \\
n & & I_{z}>100 \mathrm{~T}_{e}
\end{array}\right.
$$

and

$$
<\sigma v\rangle_{\text {rec }}^{Z}=\frac{A_{Z}^{r e c} B^{3 / 2}}{\beta+X_{X}^{r e c}}
$$

where $\beta \frac{I_{Z}}{T_{e}}$. The values of the coefficients $A_{Z}^{i o n}, A_{Z}^{\text {rec }}$ and parameters $x_{Z}^{i o n}$ and $X_{Z}^{r e c}$ for each charge state are supplied in DATA statements in the subroutine. These values were obtained by $\mathrm{R}$. A. Dory, ${ }^{3}$ who made arjustments in the results given by Beigman et $a 1 .,{ }^{2}$ and Lotz. ${ }^{4}$

Called livim:

DINAM

Variable required:

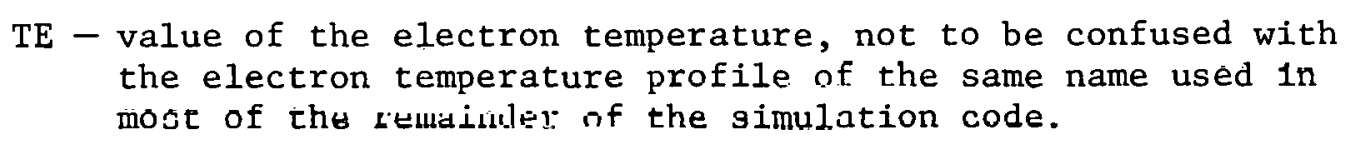

Airays changed

$$
\begin{aligned}
& \text { SVION - values of the ionization coefficient }\langle\circ\rangle^{2} \text { ion for each } \\
& \text { charge state } Z \\
& \text { SVREC - values of the recombination coefficlent cuvis rec tor each } \\
& \text { charge state } Z
\end{aligned}
$$




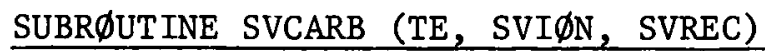

Subroutine SVCARB generates ionization $\left\langle\sigma v{ }^{\mathrm{Z}}{ }_{\text {ion }}\right.$ and recombination $<\sigma v{ }_{\text {rec }}^{Z}$ coefficients for the different charge states of carbon. These coefficients are required by subroutine DYNAM for calculating the densities of the various carbon impurity charge states. The coefficients are generated using the analytic expressions ${ }^{2}$

$$
<\sigma>_{\text {ion }}^{Z}=\left\{\begin{array}{cc}
A_{Z}^{\text {ion }} e^{-\beta} \frac{\sqrt{\beta}}{\beta+x_{Z}^{\text {ion }}} & , I_{Z} \leqslant 100 T_{e} \\
0 & , I_{Z}>100 T_{e}
\end{array}\right.
$$

and

$$
\operatorname{cov}_{\text {rec }}^{Z}=\frac{\Lambda_{Z}^{\text {rec }} e^{3 / 2}}{\beta+x_{Z}^{r e c}} \text {, }
$$

where $\beta=\frac{I_{Z}}{T_{e}}$. The values of the coefficients $A_{Z}^{i o n}, Z_{Z}^{\text {rec }}$ and parameters $\mathrm{x}_{\mathrm{Z}}^{\text {ion }}$ and $\mathrm{x}_{\mathrm{Z}}^{\mathrm{rec}}$ for each charge state are supplied in DATA statements in the subroutine. These values were obtained by $R$. A. Dory, ${ }^{3}$ who made adjustments in the results given by Beigman et al., ${ }^{2}$ and Lotz. ${ }^{4}$

Called from: DYNAM

Variable required;

$T E$ - value of the electron temperature, not to be confused with the electron temperature profile of the same name used in most of the remainder of the simulation code

Arrays changed:

SVION - values of the ionization coefficient $\langle\sigma v\rangle_{\text {ion }}^{2}$ for each
charge state $z$

SVREC - values of the recombination coefficient $\left\langle\sigma v{ }_{\text {rec }}^{Z}\right.$ for each charge state $\mathrm{Z}$ 
REFERENCES

1. P. H. Rutherford, Phys. Fluids 17: 1782 (1974).

2. I. L. Beigman, L. A. Vainshtein, and A. Vinogradov, Astron. Zh. [Sov. Astron. - AJ] 13: 775 (1970).

3. R. A. Dory, private communication.

4. W. Lotz, Astrophys. J. Supp Z. Ser. 128 14: 207 (1967). 


\section{DESCRIPTION OF NEUTRAL BEAM INJECTION MODULE}

\subsection{PURPOSE, STRUCTURE, AND BASIC INPUT/OUTPUT OF MODULE}

The neutral beam module treats the slowing down and charge-exchange interactions between the injected neutral beam and the plasma target. The module calculates the fast-ion deposition profile, the power transferred to electrons and ions in the plasma, and the change in the plasma current density. Heating and current perturbation profiles and smooth injection profiles are also obtained.

The fast-ion deposition profile $H\left(\xi=\frac{r}{a}\right)$ is calculated when the simulation time (TIM) first exceeds the time of injection turn-on (TNJCT), as shown in Fig. 3. Subroutine SUBH calculates the profile at ten radial positions $(\xi=0,0.1, \ldots, 0.9)$, and the results are stored in the array HTMP.

In order to assure a smooth beam turn-on, it is assumed that the beam has already slowed for a given time DTCGL in the simulated plasma existing at time TNJCT. This assumption is used in calculating the fractions of beam power transferred to the ions $\left(G_{i}\right)$ and to the electrons $\left(G_{e}\right)$ in the plasma, and in determining the change in the plasma current density $(\Delta J)$. These values are obtained at ten radial positions by a call to subroutine FPM $\varnothing M E$ and are stored in the arrays GETMP, GITMP, and DJTMP.

Heating and current perturbation profiles are then obtained at the ten radial positions for the time TNJCT + DTCCL by uoing

$$
Q_{e, i}=\sum_{\text {beam components, } J} \frac{I_{B} E_{B}(J) H_{J}(r) G_{e, i}(J)}{e \times \text { volume }}
$$

and

$$
\Delta \mathrm{J}=\sum_{\text {beam components, } \mathrm{J}} \Delta \mathrm{J}(\mathrm{J})
$$


The profiles are stored in arrays QETMP, QITMP, and DJTTMP. At time TNJCT, the injection profiles are all assumed to be zero.

Smooth injection profiles are then obtained over the entire number $\mathrm{N}$ of spatial grid points used in the simulation code by a fourth order spatial interpolation between the eleven calculated values for the points $r=0.0,0.1, \ldots, 0.9,1.0$, where the heating and current perturbation are assumed to be zero. These smooth profiles, calculated at the times $\mathrm{t}_{1}=$ TNJCT and $\mathrm{t}_{2}=$ TNJCT + DTNJCT, are stored in QES, QIS, and DJS. As the simulation progresses from $t_{1}$ to $t_{2}$, the heating (QE1 and QII) and current perturbation (DJT) proflles for each time are assumed to be obtained from a linear interpolation at each radial point between the values at $\tau_{1}$ and $t_{2}$.

When the simulation time $t=t_{2}$, subroutine FPMØME 1 s called to give values of $G_{e}, G_{i}$, etc. for $t_{3}=$ TNJCT $+2 *$ DTCGL, and a new set of profiles is generated for this time. The temporal interpolation-is now between the old values of the injection profiles calculated for $t_{2}$ and the values just calculated at $t_{3}$ as the simulation proceeds from $t_{2}$ to $t_{3}$. This procedure repeats every DTCGL milliseconds until the end of the simulation. Every DTCH miliseconds, starting at $t=$ 'I'NJC'l', subroutine SUBH is called to generate a new fast-ion deposition profile $\mathrm{H}(\xi)$ which is used for each injection profile until the next call to SUBH. Flgure 8 shows the order of subroutine calls in this module.

\subsection{SUMMARY OF FUNCTION OF EACH SUBROUTINE}

BI $\emptyset \mathrm{CK}$ DATA (FOR THE NEUTRAL B̈ËAM INJECTIUN MUDULE)

The block data subprogram for the neutral beam injection module contains initial values for the loss cone correction arrays. The array names and subscripts have the general form STX ( $I, J, L, M$ ), where the values of $X, I, J, L$, and $M$ are given in Table 13 with a brief description of each case represented by the values of these characters. The numbers contained in the loss cone correction arrays were generated using a Fokker-P1anck code. ${ }^{1}$ 
ORNL-OWG 76-2310

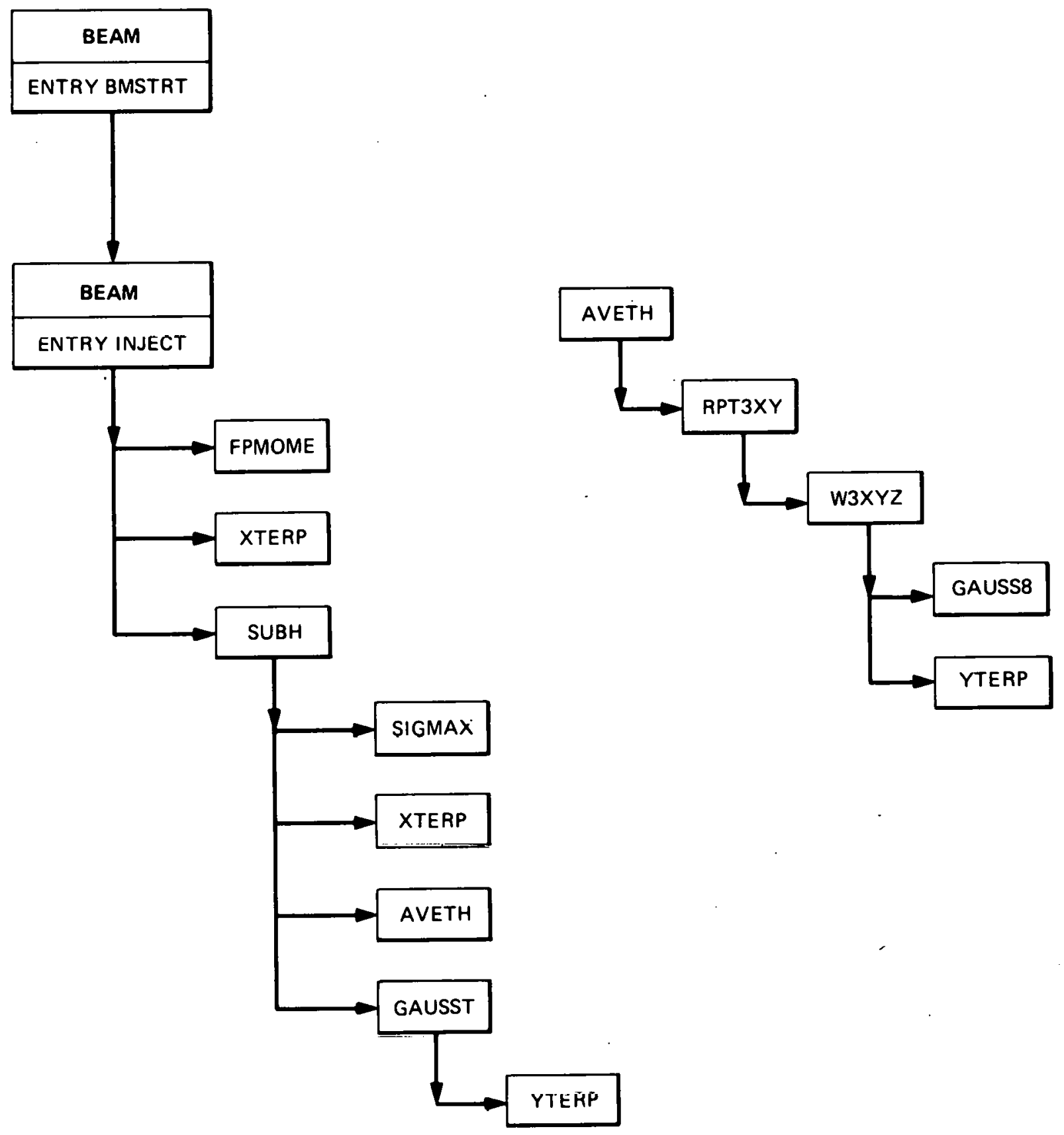

Fig. 8. Order of primary subroutine calls in the neutral beam injection module. 
Table 13. Identification of characters used to label loss cone correction arrays $\operatorname{STX}(\mathrm{I}, \mathrm{J}, \mathrm{L}, \mathrm{M})$

\begin{tabular}{|c|c|c|c|}
\hline & $\underline{x}^{a}$ & $\underline{\mathrm{K}}$ & Description \\
\hline & 1 & 1 & $G_{e}$, no luss cone correction \\
\hline & 2 & 1 & $G_{i}$, no loss cone correction \\
\hline & 3 & 1 & $\mathrm{KE}$, no loss cone correction \\
\hline & 4 & 1 & $G_{e}$, with loss cone correction \\
\hline & 5 & 2 & $\mathrm{C}_{i}$, with 1033 cone correction \\
\hline & 6 & 2 & KE,' with luss culle cuilecliull \\
\hline Subscript $^{a}$ & & $\begin{array}{c}\text { Range of } \\
\text { Values } \\
\end{array}$ & Identification \\
\hline I & & 1,10 & $\begin{array}{l}\text { Ten values of the radius (normalized): } \\
0.0,0.1, \ldots, 0.9\end{array}$ \\
\hline $\mathrm{J}$ & & 1,4 & $\begin{array}{l}\text { Energy components of injected neutral } \\
\text { beam: } \mathrm{E}_{\mathrm{o}}, \mathrm{E}_{\mathrm{o}} / 2, \mathrm{E}_{\mathrm{o}} / 3 \text {, and the sum of } \\
\text { all three }\end{array}$ \\
\hline $\mathrm{K}$ & & 1,2 & Loss cone corrections: without, with \\
\hline L & & 1,2 & Injection direction: co-, counter- \\
\hline M & & 1,2 & Impurity type: low-, high-z \\
\hline$\dot{N}$ & & 1,2 & Power component: $G_{e}, G_{i}, K E$ \\
\hline
\end{tabular}

$a_{\text {Data }}$ is stored in the arrays in BL $\emptyset \mathrm{CK}$ DATA as STX $(\underline{I}, \underline{J}, \underline{L}, \underline{\mathrm{M}})$ 


\section{SUBRØUTINE BEAM}

Subroutine BEAM contains the primary logical control for the neutral beam injection module. Figure 9 shows this primary logical control and indicates where some of the computations described in Sect. 6.1 are done. Switch variables are also listed with the options which may be chosen according to the values assigned to these variables. The subscripts II, $\mathrm{J}$, and $\mathrm{L}$ used in the output arrays refer to the radial position, energy component, and type of neutral beam injection, whether co-injection $(L=1)$ or counter-injection $(L=2)$.

In the alternate ENTRY BMSTRT section of the subroutine, many of the variables used in the moments calculation of $G_{e}, G_{i}, G_{e}^{E}, G_{i}^{E}$, etc., are initialized. Variables and arrays used to calculate the fast-ion deposition profile are initialized next, followed by adjustments (if necessary) to the loss cone correction tables. Logic near the beginning of this alternate entry section routes control to the remaining subsections.

The labeled common blocks associated primarily with the moments caiculation are INPUT and ØUTPUT. Commons FPPP and INTRP are used primarily in the $\mathrm{H}(\mathrm{r})$ calculation.

Alternate ENTRY INJECT contains the logic and the interpolation and numerical procedures for calculating the heating ańd current perturbation profiles from the results of the moments and $H(r)$ calculations for each simulation time step.

Due to the relatively long time required to do the injection computations, the complete heating and current perturbation profiles are computed at time intervals much longer than the simulation code time step. Subroutine BEAM interpolates between calculated values of the injection profiles for spatial and time grids which are coarser than the grids required by the rest of the simulation code.

In the call to alternate entry BMSTRT, the switch setting $\operatorname{INJ}(1)=2$ can be used to initialize the injection parameters quickly, but with only approximate values. The profiles for the electron and ion neutral beam heat input are assumed to have a decreasing exponential shape. This feature is useful for debugging the program. 


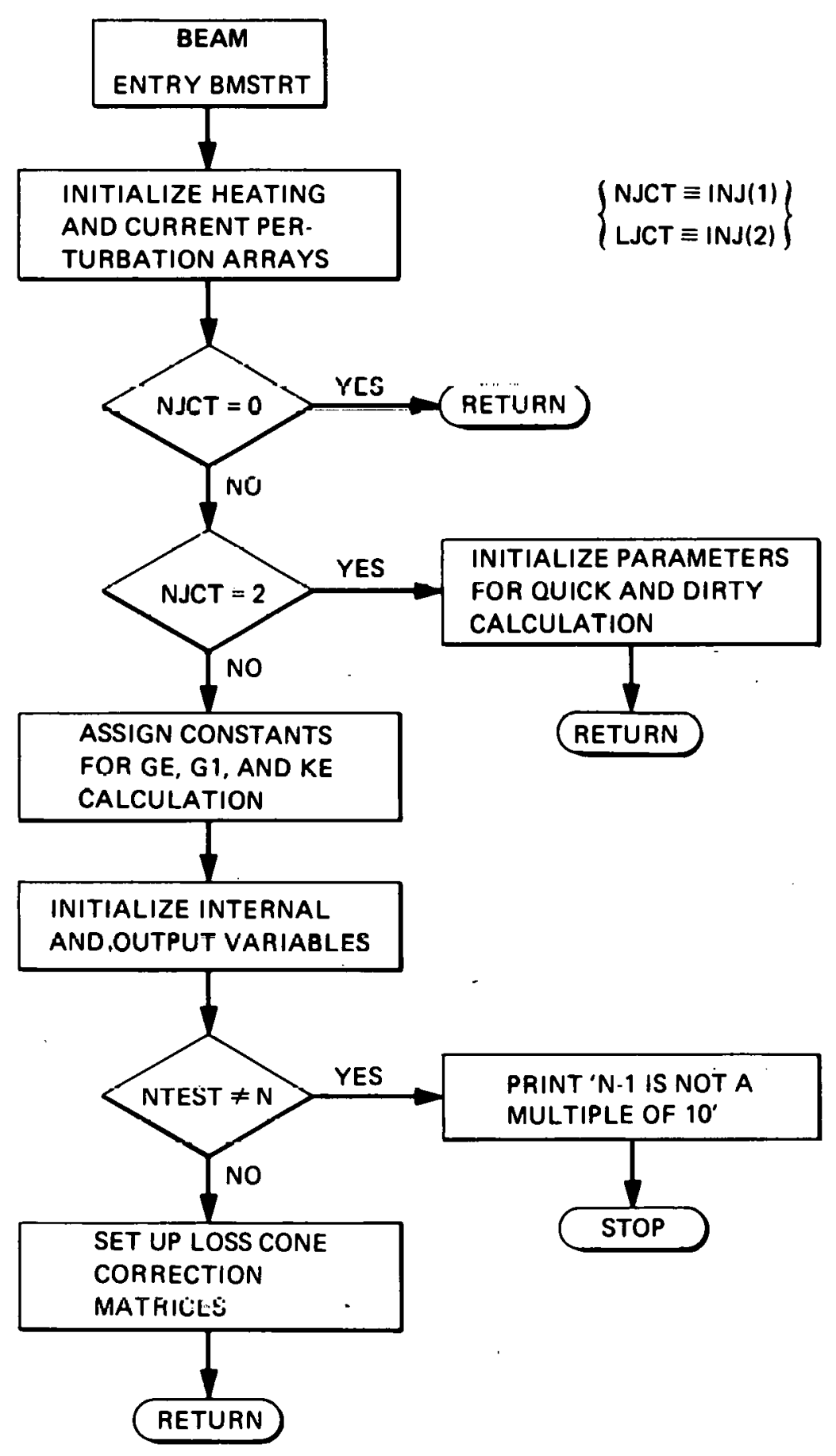

Fig. 9a. Primary logic for subroutine BEAM, ENTRY BMSTRT (initialization of arrays for neutral beam injection simulation). 


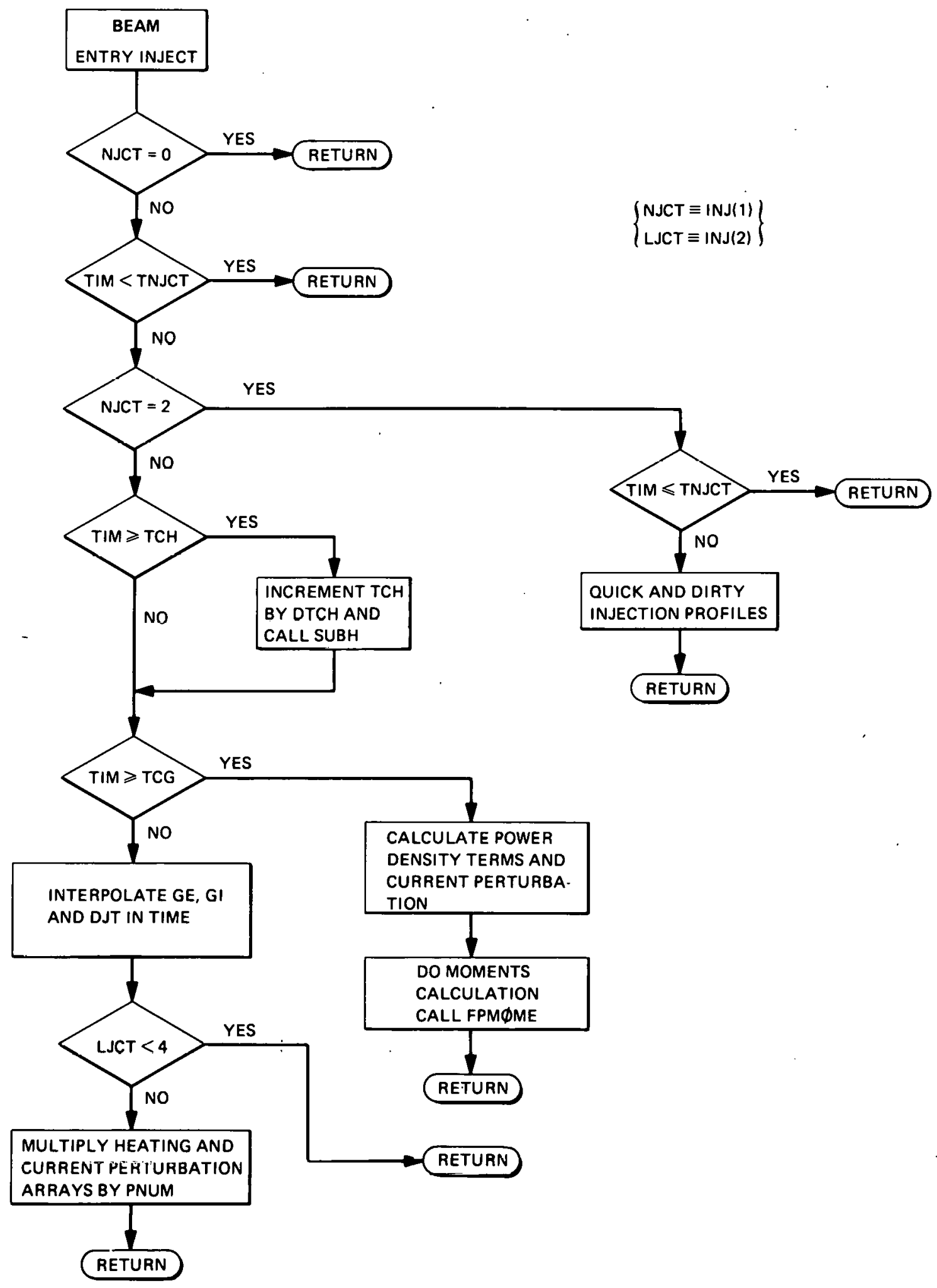

Fig. 9b. Primary logic for subroutine BEAM, ENTRY INJECT (main part of the calculation of the neutral beam injection module). 
Called from:

Subroutines called:

Commons required:

Variables and arrays required:

Variables and arrays changed:
GETSET (BMSTRT), MAIN (INJEC'I)

FPMØME, XTERP, SUBH

BEAMC, BEAMP, ELCTRN, FIELDS, FPPP, FUDGE, GE $\emptyset$ M, IMPURT, INDEX, INPUT, INTRP, IØNS, NEUTRL, ØUTPUT, TEMP, TIME, TMINDX, $\mathrm{XP} \emptyset \mathrm{RT}$

See Table 14 and Appendix II

See Table 14 and Appendix II 
Table 14. List of labeled commons and elements in them used by subroutine BEAM

\begin{tabular}{|c|c|c|c|c|}
\hline \multirow{2}{*}{$\mathrm{C} \emptyset \mathrm{MM} \emptyset \mathrm{N} \quad \mathrm{BL} \emptyset \mathrm{CK}$} & \multicolumn{2}{|c|}{ Input } & \multicolumn{2}{|c|}{$\emptyset$ utput } \\
\hline & Variables & Arrays & Variables & Arrays \\
\hline /BEAMC/ & $\begin{array}{l}\text { ENGY (1), DTCH, DTCGL, } \\
\text { RC, TNJCT, NJCT, LJCT }\end{array}$ & CURBM & $\operatorname{ENGY}(2), \operatorname{ENGY}(3)$ & DJT \\
\hline /BEAMP / & TCGS (2) & & $\begin{array}{l}\text { TCGS (1), PNUM, } \\
\text { TCHS, MM }\end{array}$ & $\begin{array}{l}\text { HTMP, GETMP, GITMP, } \\
\text { DJTMP, QETMP, QITMP, } \\
\text { DJTTMP, SDT, ECRS, } \\
\text { DJSUM, HX }\end{array}$ \\
\hline /ELCTRN/ & $\mathrm{FQE}$ & & & \\
\hline /FIELDS/ & & $\mathrm{E}$ & & \\
\hline /FPPP/ & & & NCGLM & \\
\hline /FUDGE/ & & $\begin{array}{l}\text { STL, ST2, ST } 3 \\
\text { ST4, ST5, ST6 }\end{array}$ & & ST4, ST5, ST6 \\
\hline /GEØM/ & $\mathrm{AM}, \mathrm{R} \emptyset, \mathrm{V} \emptyset \mathrm{L}$ & & & \\
\hline / IMPURT/ & & $\mathrm{ZBR}$ & & \\
\hline /INDEX/ & $\mathrm{N}, \mathrm{NT}$ & INJ & & \\
\hline /INPUT/ & & & $\begin{array}{l}\text { T, EФ, TEE, TII, } \\
\text { DNE, DNO, AMF, } \\
\text { AMI, BRAZ, SQBRZ, } \\
\text { ZF, ETO, EPA3, } \\
\text { EPA4, EPA5, M3, } \\
\text { M4, M5, ICTX, INO }\end{array}$ & . \\
\hline
\end{tabular}


Tatle 14 (continued)

\begin{tabular}{|c|c|c|c|c|}
\hline \multirow{2}{*}{$C \emptyset M M \varnothing N \quad B L \varnothing C K$} & \multicolumn{2}{|c|}{ Input } & \multicolumn{2}{|c|}{$\emptyset$ utput } \\
\hline & Vari $\exists b 1 \equiv s$ & Arrays & Vaziab_es & Arrays \\
\hline /INTRP/ & $\mathrm{T} \ddot{\mathrm{Y} L} \mathrm{~L}$ & $x x x$ & & RTMP \\
\hline /IONS/ & & DEN & & \\
\hline /NEUTRLi & & $\operatorname{zN} 1$ & & QE1, QI1 \\
\hline /OUTPUTi & $\begin{array}{l}\text { EC } \emptyset, \operatorname{TS} \phi, \operatorname{TF} \phi, \\
\text { TCXV } \emptyset, \text { VO } \emptyset, \\
\text { GII, GEE, BKE, } \\
\text { GIE, GEEE, BKEE }\end{array}$ & & & \\
\hline /TEMP/ & & $\mathrm{TE}, \mathrm{TI}$ & & \\
\hline /TIME/ & HT, TIM, TMAX & & & \\
\hline /TMINDX, & DTNJCT, N1, N3 & & & \\
\hline /XPøRT/ & & Dl & & \\
\hline
\end{tabular}


$\underline{\text { SUBRØUTINE BMPWR }}$

Subroutine BMPWR uses information calculated in the neutral beam injection module to generate profiles showing the power balance for the injected neutral beam after it has interacted with the target plasma. Table 15 shows the variables and arrays required as input and generated as output.

In all cases, $1 \leqslant J \leqslant 3$ refers to the energy component $\left(E_{0}, E_{o} / 2\right.$, or $\left.E_{o} / 3\right)$ of the beam and $L=1,2$ to whether co- or counter-injection is used. The subroutine calculates total power by suming beam components and fractions of the total deposited power which go to electrons, ions, and the wall. The component and total current perturbations are also calculated. This information is printed by the subroutine when the printout for the rest of the simulation code is requested.

Called from: $\quad$ MAIN

Subroutines called: QUADR

Commons required: $\quad$ BEAMC, BEAMP, FPPP, GEøM, INDEX, INTRP, NEUTRL, TIME; XTRA

Arrays changed:

FNBA - fraction of injected neutral beam deposited in the plasma (neutral beam absorption factor)

FPRT - fractions of total injected neutral beam power deposited in the plasma electrons and ions, lost to charge exchange, and deposited directly into the liner

DIT - sum of contributions to current perturbation resulting from co- and counter-injected neutral beams

DITT - sum of current perturbation contributions resulting from the three injected beam energy components

Variables representing amounts of power, $\mathrm{W} / \mathrm{cm}^{3}$, for each energy component and injection configuration.

QTB - supplied by the neutral beam injector

QTD - deposited in the plasma

QEB - deposited in the plasma electrons

QIB - deposited in the plasma ions

QCX - lost to charge exchange

QBL - deposited directly into the liner 
Variables representing total power, $W / \mathrm{cm}^{3}$, for the sum of the three injected beam energy components

QTBT - supplied by the neutral beam injector

QTDT - deposited in the plasma

QEET - deposited in the plasma electrons

QIIT - deposited in the plasma ions

QCXT - lost to charge exchange

QBLT - deposited directly into the liner

QINT - a working array containing intermediate quantities rclated to neutral beam power 1nss or transfer and current perturbation

SS - value of integral of profile contained in QINT array, computod by call to suthrnirine RIJADR 
Table 15. List of labeled commons and elements in them used by subroutine BMPWR

\begin{tabular}{|c|c|c|c|c|}
\hline \multirow{2}{*}{$C \emptyset M M \emptyset N B L \emptyset C K$} & \multicolumn{2}{|c|}{ Input } & \multicolumn{2}{|c|}{$\emptyset$ utput } \\
\hline & Variables & Arrays & Variables & Arrays \\
\hline /BEAMC/ & HJCT, LJCT & CUREM, ENGY & & \\
\hline /BEAMP / & PNUM, $\mathrm{TCHS}^{\alpha}, \mathrm{MM}^{a}$ & $\begin{array}{l}\text { HTMP, GETMP, GITMP, } \\
\text { DJTMP, DJTIMP } a \\
\text { TCGS, } a \text { SDT }, a \text { ECRS }, a \\
\text { DJSUM, } a \text { XPRT } a\end{array}$ & & $\begin{array}{l}\text { FNBA, QEB, QIB, QCX, } \\
\text { QTB, QTD, QBL, QTBT, } \\
\text { QTDT, QEET, QIIT, } \\
\text { QCXT, QBLT, DIT, } \\
\text { DITT, FPRT }\end{array}$ \\
\hline /FPPP/ & & $\mathrm{FPCX}, a$ FPI, ${ }^{a} \mathrm{FPE},{ }^{a} \mathrm{FP} a$ & & \\
\hline /GEøI / & $\mathrm{AM}$ & & & \\
\hline /INDEX/ & $\mathrm{N}$ & & & \\
\hline /INTRP/ & & RTMP & & \\
\hline /NEUTRL/ & & $\mathrm{QE} 1,{ }_{\mathrm{QII}}^{a}$ & & \\
\hline /TIME/ & $\operatorname{TIM}^{\alpha}$ & & & \\
\hline /XTRA/ & & $\operatorname{TE} 12^{a}$ & & \\
\hline
\end{tabular}

\footnotetext{
¿variatles and arrays used only for printout.
} 
SUBRØUTINE QUADR $(\mathrm{Z}, \mathrm{N}, \mathrm{H}, \mathrm{S}, \mathrm{IER})$

Subroutine QUADR integrates a function by numerical quadrature using a basic five-point formula if there are at least five function values. The routine assumes that the values of the independent variable (function argument) are equally spaced. Since the five-point formula uses successive groups of five function values, there will be function values remaining at one end of the integration interval when $\mathrm{N}(\bmod 4) \neq 0$. The integration is completed by a 2-, 3-, or 4-point formula according to whether $\bar{N}(\bmod 4)$ is 1,2 , or 3.

Called from: BMPWR

Variables required:

$$
\begin{aligned}
& \mathrm{Z}(50) \text { - array of values of function to be integrated } \\
& \mathrm{N} \text { - number of points for which function values exist } \\
& \mathrm{H} \quad \text { - spacing between successive values of the independent } \\
& \text { variable }
\end{aligned}
$$

\section{Variables changed:}

$S$ - value of the integral

IER - diagnostic and error variable

$=0$, normal return

m 1 , only a single value or no value of the function to be integrated was passed from the calling program

$=2$, the interval between successive values of the independent variable vanishes 


\section{SUBRØUTINE SUBH}

Subroutine $\mathrm{SUBH}$, when it is first called, initializes the variables and arrays,required by the subroutines which generate the fast-ion deposition profile $H(r) .{ }^{2}$ The values of these variables and arrays are stored in the common block areas $\mathrm{CN}$ and D. These initializations are skipped on subsequent calls.

For each neutral beam energy component this subroutine calculates the mean free paths of the injected ions due to charge exchange and ionization. It sets up the plasma density profile (normalized to the central density) used in the calculation of $\mathrm{H}(\mathrm{r})$ and calls the subroutines to compute

$$
H(r)=\frac{a^{2}}{I_{T} \pi r_{B}^{2}} \int_{0}^{\pi} d \theta \int_{0}^{\min }\left\{\begin{array}{l}
\rho \\
r_{B}
\end{array}\right\} \frac{d Z_{B}}{\sqrt{\rho^{2}-Z_{B}^{2}}}\left[I^{+}+I^{-}\right],
$$

where

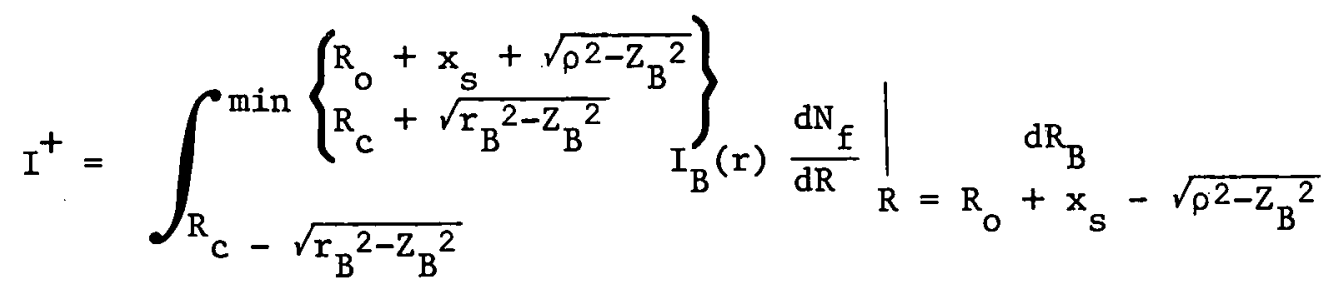

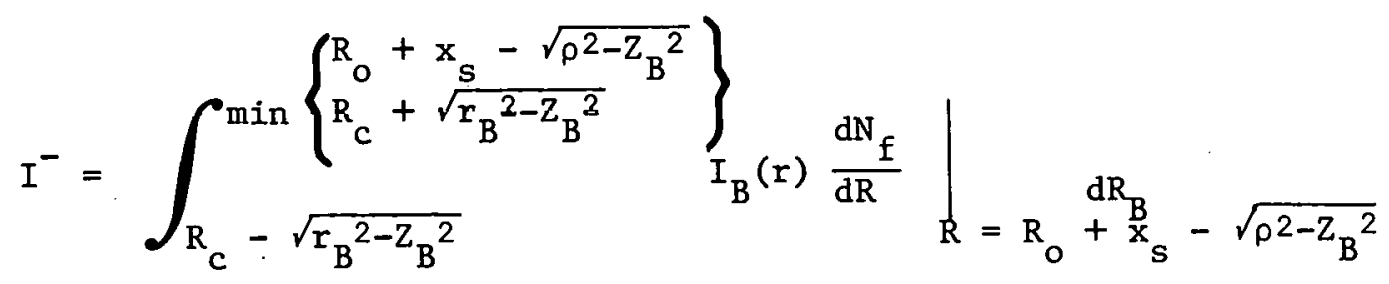

$$
\begin{aligned}
& \frac{\mathrm{dN}_{\mathrm{f}}}{\mathrm{dR}}=\frac{1}{\tilde{\lambda}_{0}} \frac{\tilde{\mathrm{n}}(\mathrm{r})}{\tilde{\mathrm{n}}(\mathrm{o})} \frac{\mathrm{R}}{\sqrt{\mathrm{R}^{2}-\mathrm{R}_{\mathrm{B}}^{2}}} \exp \left(\frac{1}{\lambda_{0}}\right) \int_{\mathrm{R}_{0}+\sqrt{\mathrm{a}^{2}-\mathrm{Z}_{\mathrm{B}}^{2}}}^{\mathrm{R}} \\
& \left.\left.+\int_{R}^{R_{B}}-\int_{R_{B}}^{R_{0}+\sqrt{a^{2}+Z_{B}^{2}}}\right]_{\frac{\tilde{n}(r)}{\tilde{m}(0)}} \frac{R^{\prime} d R^{\prime}}{\sqrt{R^{\prime 2}-R_{B}^{2}}}\right\}
\end{aligned}
$$




$$
\rho=\sqrt{r^{2}+x_{s}^{2}-2 r x_{s} \cos \theta} \leqslant a-x_{s},
$$

and

$$
x_{S}=229.581[1-2(L-1)] \frac{\sqrt{\operatorname{ENGY}(J)}}{R_{0}^{* Z J(1)}},\left\{\begin{array}{l}
\mathrm{L}=1, \text { co-injection } \\
\mathrm{L}=2, \text { counter-injection } \\
\mathrm{J}=1,2,3 \text { for the three } \\
\text { energy components of the } \\
\text { injected neutral beam. }
\end{array}\right.
$$

Subroutine SUBH calculates the plasma density profile used in the expression for $\mathrm{H}(\mathrm{r})$ at the $\mathrm{N}$ grid points used by the rest of the simulatinn rode. The subroutine then calls a routine XTERP to do a fourth order interpolation to generate a table of values for 101 equally spaced radial points. However, the spatial meshes of the integrations required to generate the profile $\mathrm{H}(\mathrm{r})$ and uscd in the simulation code are independent of each other.

The quantity $\frac{1}{\tilde{\lambda}_{0}} \frac{\tilde{n}(r)}{\tilde{n}(0)}$ used here is different in form and kind from that used by Colchin and Wooten. ${ }^{2}$ Some additional physics, the effect of impurities, has been added by defining a cross section for impurities $\sigma_{I}=z_{I}^{2} \sigma_{\rho}$ for each impurity species $I$. The cross section $\sigma_{\rho}$ is detined in terms of the proton impact 1onlzallun leciprocal mcan free path $\langle\sigma v\rangle=\sigma_{\rho} v_{o}$, where $v_{0}$ is the infrlal velucity of the injected neutral beam.

Using the definition for $\mathrm{Z}_{\text {eff }}$, the collision frequency,

$$
v_{T}=n_{e}\langle\sigma v\rangle_{e}+n_{i}\left(\langle\sigma v\rangle_{\rho}+\langle\sigma v\rangle_{c x}\right)+\sum_{I} L_{I} I_{i}{ }^{\prime}\langle\sigma v\rangle_{\rho},
$$

can be written as

$$
\nu_{T}=v_{0} n_{i}(0) \sigma_{c x}\left[\frac{n_{e}(r)}{n_{i}(0)} \frac{\sigma_{v v}}{\sigma_{c x} v_{0}}+\frac{n_{i}(r)}{n_{i}(0)}+\frac{n_{e}(r)}{n_{i}(0)} \frac{\sigma_{p} z_{e f f}}{\sigma_{c x}}\right] \text {. }
$$

Letting

$$
\lambda_{c x}=\frac{1}{n_{i}(0) \sigma_{c x}}, \quad \lambda_{i}=\frac{1}{n_{i}(0) \sigma_{\rho}}, \quad \text { and } \lambda_{e}=\frac{v_{0}}{\left.n_{e}(0)<\sigma v\right\rangle_{e}},
$$


then

$$
\frac{1}{\lambda_{T}}=\frac{1}{\lambda_{c x}}\left\{n_{i}(r)+n_{e}(r)\left(\frac{\lambda_{c x}}{\lambda_{i}} z_{e f f}+\frac{n_{i}(0)}{n_{e}(0)} \frac{\lambda c x}{\lambda_{e}}\right)\right\} \frac{1}{n_{e}(0)}
$$

and the absorption decrement can be written as

$$
D(s)=-\int_{S_{0}}^{S} \frac{d s}{\lambda_{T}}=\frac{1}{\lambda_{c x}(0)} \int_{S_{0}}^{S} \frac{\lambda_{c x}(0)}{\lambda_{T}} d s .
$$

Thus $\quad \tilde{\lambda}_{0}=\lambda_{c x}(0), \tilde{n}(0)=n_{e}(0)$

and

$$
\tilde{n}(r)=n_{i}(r)+n_{e}(r)\left(\frac{\lambda_{c x}}{\lambda_{i}} z_{e f f}+\frac{n_{i}(0)}{n_{e}(0)} \frac{\lambda_{c x}}{\lambda_{e}}\right) .
$$

The stagnation distance $x_{s}$ (assumed constant) is also calculated for each neutral beam energy component. Subroutine SUBH then calls subroutine AVETH to get the $H(r)$ profile, which is symmetric about the stagnation point. Subroutine GAUSST averages $\mathrm{H}(\rho, \theta)$ on a flux surface to give $H(r)$. A separate $H(r)$ profile is calculated for each of the three beam energy components for co- and/or counter-injection. Thus, as many as $\operatorname{six} H(r)$ profiles can be returned for each call to subroutine SUBH; these profiles are stored in the array HTMP.

Called from: $\quad$ BEAM (alternate ENTRY INJECT)

Subroutines called: SIGMAX, XTERP, AVETH, GAUSST

Commons required: $\quad$ BEAMC, BEAMP, CN, D, E, FIELDS, FPPP, GE $\emptyset$, IMPURT, INDEX, INTRP, I $\emptyset \mathrm{NS}, \mathrm{TA}, \mathrm{TEMP}, \mathrm{XP} \emptyset \mathrm{RT}$

Variables and arrays required: See Table 16 and Appendix II

Variables and arrays changed: See Table 16 and Appendix II 
Table 16. Iist of labeled commors anc elements in them used by subroutine SUBH

\begin{tabular}{|c|c|c|c|c|}
\hline \multirow{2}{*}{$\mathrm{C} \emptyset \mathrm{MM} \emptyset \mathrm{N} \quad \mathrm{BL} \emptyset \mathrm{CK}$} & \multicolumn{2}{|c|}{ Input } & \multicolumn{2}{|c|}{$\emptyset$ utput } \\
\hline & Variajles & Arrays & Variables & Arrays \\
\hline /BEAMC/ & R7, R8, HA匚F, L.JCT & ENGY & & \\
\hline /BEAMP / & & & & HTMP, XPRT \\
\hline$/ \mathrm{CN} /$ & & & $\mathrm{SI}, \mathrm{NP}$ & $\mathrm{s}$ \\
\hline$/ \mathrm{D} /$ & & & $\begin{array}{l}A, X S, 3 B, R=\emptyset \\
R C, 30, \emptyset F, N Z\end{array}$ & \\
\hline /FIELDS/ & & $2 J$ & & \\
\hline /FPPP/ & NCGLM & & & $\begin{array}{c}\text { FPCX, FPI, FPE, } \\
\text { FP, }\end{array}$ \\
\hline$/ \mathrm{GE} \emptyset \mathrm{M} /$ & $\mathrm{AM}, \mathrm{R} \emptyset$ & & & \\
\hline / IMPURT/ & & DENP & & \\
\hline /INDEX/ & $\mathrm{N}$ & & & \\
\hline / INTRP/ & & $\mathrm{xxx}$ & $\mathrm{T} \emptyset \mathrm{L}$ & PIDEN \\
\hline /IØNS/ & & $\mathrm{D} \Xi \mathrm{N}$ & & \\
\hline$/ \mathrm{TA} /$ & & HTT & $\begin{aligned} \text { RHøMAX, } & \text { DELI } \\
\text { DELN, } & \text { NGLP1 }\end{aligned}$ & $\mathrm{HRH} \emptyset, \mathrm{RV}$ \\
\hline /TEMP/ & & $\mathrm{TE}$ & & \\
\hline$/ \mathrm{XP} \emptyset \mathrm{RT} /$ & & $\mathrm{D} 1$ & & \\
\hline
\end{tabular}


SUBRфUTINE AVETH ( $R$, HR)

Subroutine AVETH generates the profile $\mathrm{H}[\rho(\theta)]$ using a 12-point Gauss-Chebyshev quadrature formula:

$$
\mathrm{H}[\rho(\theta)]=\frac{\pi \mathrm{A}^{2}}{9.8695 \mathrm{r}_{\mathrm{B}}{ }^{2}} * \text { ANSZ } * \mathrm{BSHAPE}
$$

where

and

$$
\text { BSHAPE }=\left(\frac{r_{B}}{H A L F}\right)^{2}\left\{1-\exp \left[-\left(\frac{r_{B}}{H A L F}\right)^{2}\right\}^{-1}\right.
$$

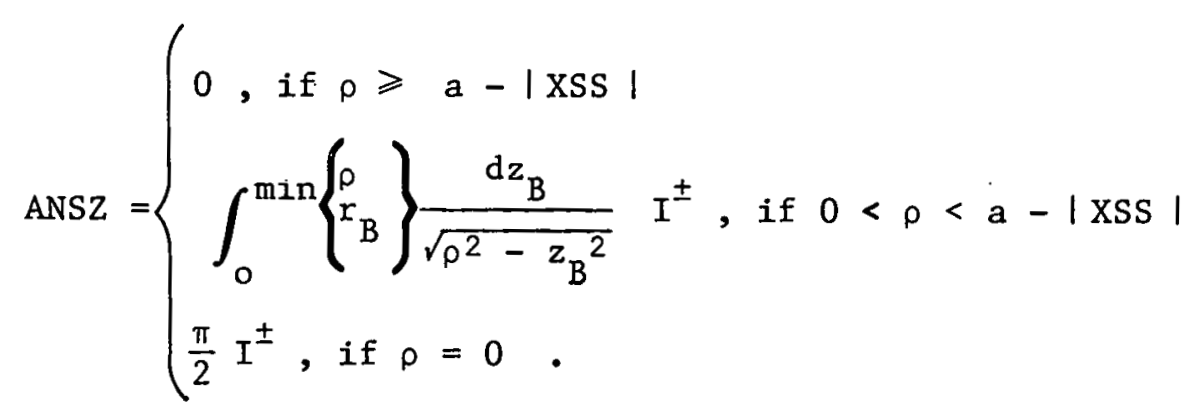

The integrals $\mathrm{I}^{+}$and $\mathrm{I}^{-}$are computed in subroutine RPT3XY according to the value of the switch $\mathrm{NZ}(=1$ and $=2$, respectively):

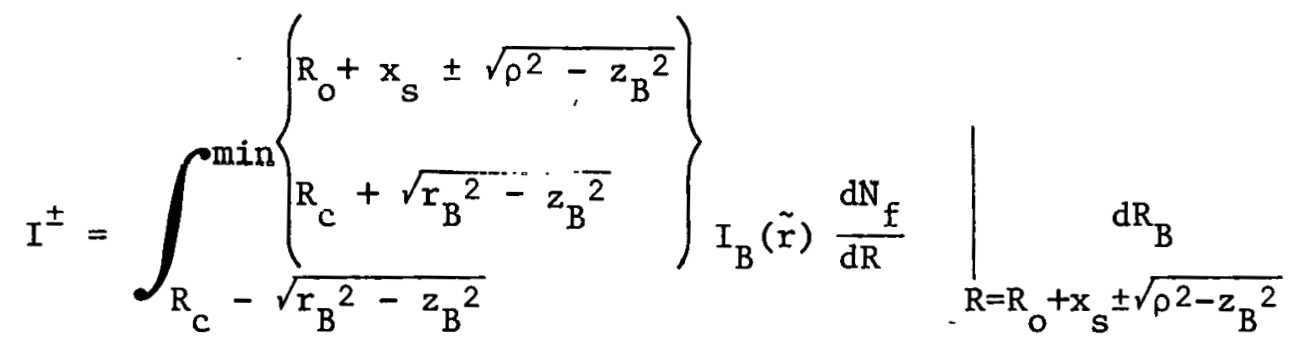

An explanation of the modifications required to get the integral over $z_{B}$ into the form required for applying the Gauss-Chebyshev quadrature formula is given in the description of subroutine RPT3XY. The constraint $\rho \geqslant a-|X S S|$ takes into account the excluded volume shown in Fig. 10.

Called from SUBH

Subroutine called: RPT $3 X Y$

Communs required: $\mathrm{CN}, \mathrm{D}, \mathrm{E}$ 

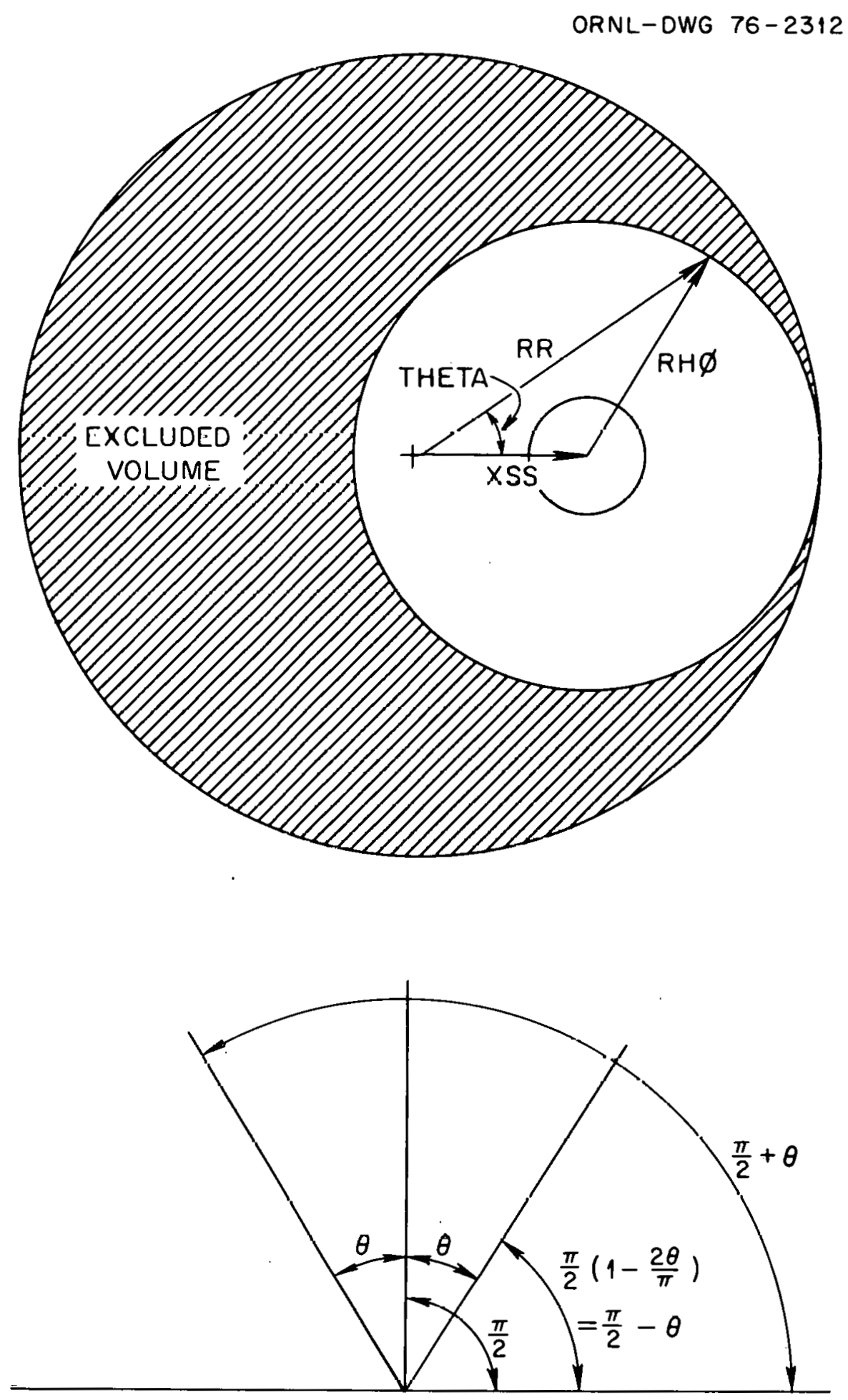

Fig. 10. Geometry and conventions used for computations in subroutines GAUSST, AVETH, and RPT3XY. 


\section{Variables required:}

$\mathrm{R}-$ - distance from the stagnation point to points in the plasma defined by $R \leqslant A-|X S S|$, where $A$ is the torus minor radius

$S$ - array of zeros of the Chebyshev polynominals of degree NP

S1 - Christoffel numbers $\beta_{j}$ for the Gauss-Chebyshev formula, the coefficient $\pi / n$ for the sum on the right-hand side (see description of subroutine RPT $3 X Y$ )

NP - the number of points used by the quadrature formula

A - torus minor radius, $\mathrm{cm}$

XSS - displacement between the magnetic axis and the stagnation point (see Fig. 10), cm

$\mathrm{RB}$ - radius $\mathrm{r}_{\mathrm{B}}$ of the injected neutral beam, $\mathrm{cm}$

$\emptyset \mathrm{FP}-$ reciprocal of the absorption mean free path of the neutral beam, $\mathrm{cm}^{-1}$

BSHAPE - injected neutral beam profile factor

Variables changed:

$$
\begin{aligned}
& \text { HR - the value of } H[\rho(\theta)] \text { for a given value of } \rho(\theta)=R \\
& R H \emptyset \text { - the value of } \rho(\theta)
\end{aligned}
$$

Significant internal variables:

$\mathrm{X}$ - the successive values of the integration variable corresponding to the zeros of the Chebyshev polynominals of degree NP

UP - upper limit of integration

$\mathrm{XL} \quad-$ lower limit of integration

RES1 - values of the integrals $I^{ \pm}$ 
SUBRøUTINE RPT 3XY (ZB, XINT3)

Subroutine RPT $3 X Y Z$ uses a 12-point Gauss-Chebyshev quadrature formula to evaluate the integrals

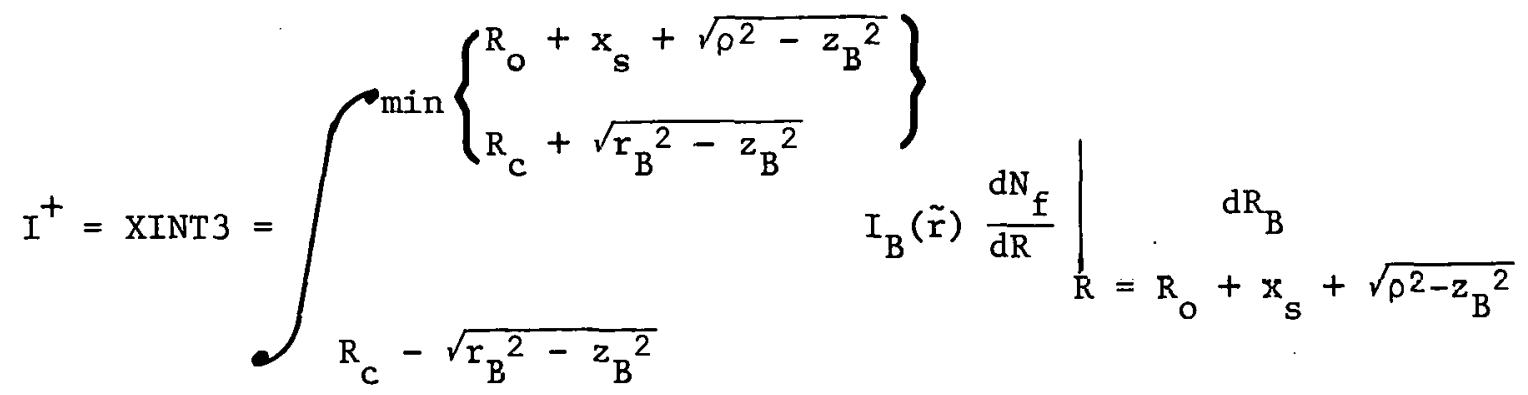

when NZ - 1 and

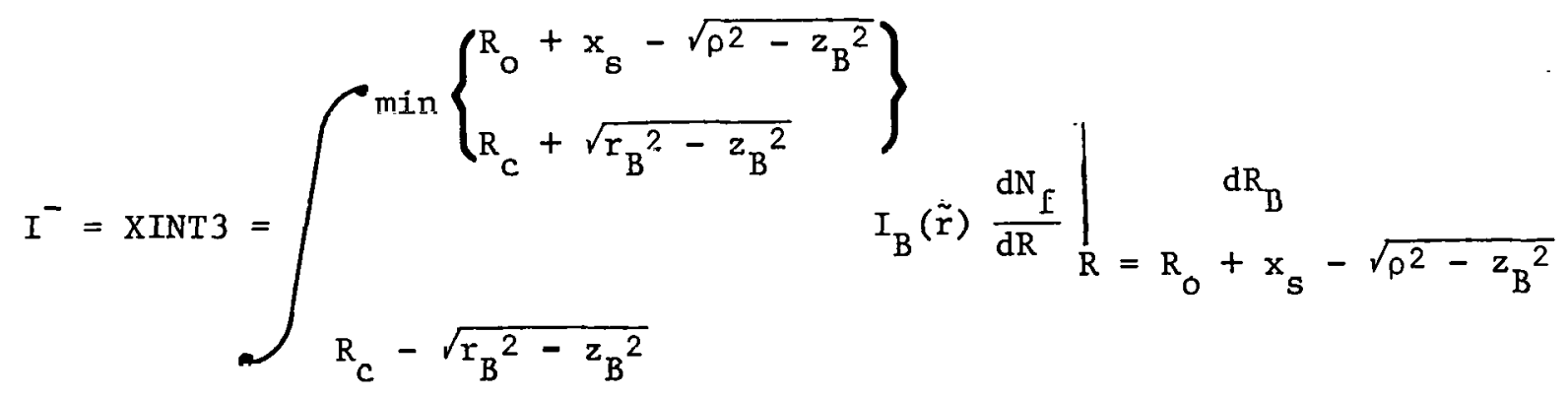

when $\mathrm{NZ}=2$. The variable $\tilde{\mathbf{r}}$ is the radial distance from the center of the injected neutral beam to the pencil beam axis. If

$$
\min \left\{\begin{array}{l}
R_{0}+x_{S} \pm \sqrt{\rho^{2}-z_{B}^{2}} \\
R_{C}+\sqrt{r_{B}{ }^{2}-z_{B}{ }^{2}}
\end{array}\right\} \leqslant R_{C}-\sqrt{r_{B}{ }^{2}-z_{B}{ }^{2}},
$$

then XINT3 $=0$. This condition takes into account the cases when the injected neutral beam does not cross a particular flux surface and hence does not contribute any fast ions to that surface.

The integrals $I^{+}$and $I^{-1}$ are of the form $\int_{b}^{a} f(x) d x$ and must be modified to obtain the form required for the Gauss-Chebyshev quadrature in the following way:

$$
\int_{a}^{b} w(x) \frac{f(x)}{w(x)} d x=\int_{a}^{b} \frac{F(x)}{\sqrt{1-x^{2}}} d x,
$$


where $w(x)=\frac{1}{\sqrt{1-x^{2}}}$ is the weight function. The Gauss-Chebyshev formula is $^{3}$

$$
\int_{-1}^{1} \frac{F(z)}{\sqrt{1-z^{2}}} d z=\frac{\pi}{n} \sum_{j-1}^{n} F\left(z_{j}\right)
$$

where

$$
F\left(z_{j}\right)=\sqrt{1-z_{j}^{2}} f\left(\frac{a+b}{2}+z_{j} \frac{b-a}{2}\right)=\sqrt{1-z_{j}^{2}} f\left(\zeta_{j}\right)
$$

The $z_{j}$ are the zeros of the Chebyshev polynominals of degree $n$.

Called from: $\quad$ AVETH

Subroutines called: W3XYZ

Commons required: $\quad \mathrm{CN}, \mathrm{D}, \mathrm{E}$

Variables required:

$\mathrm{ZB}$ - distance $z_{B}$ from torus plane of bilateral symmetry to axis of pencil beam, cm

$S$ - array of zeros $z_{j}$ of the Chebyshev polynominals of degree NP

$S 1$ - Christoffel numbers $\beta_{j}$ for the Gauss-Chebyshev formula, the coefficient $\pi / \mathrm{n}$ for the sum on the right-hand side

$\mathrm{NP}$ - the number of points used by the quadrature formula

XSS - displacement between the magnetic axis and the stagnation point (see Fig. 10), cm

$\mathrm{RB}$ - radius $\mathrm{r}_{\mathrm{B}}$ of the injected neutraL beam, $\mathrm{cm}$

$\mathrm{RH} \emptyset$ - distance from the stagnation point to points in the plasma defined by $\mathrm{RH} \emptyset \leqslant \mathrm{AM}-|\mathrm{XSS}|$, where AM is the torus minor radius

$\mathrm{RC}$ - radius of center of injected neutral beam measured from torus vertical axis, cm

RO - major radius of the torus, $\mathrm{cm}$

$\mathrm{NZ}$ - switch used to determine which integral, $\mathrm{I}^{+}$or $\mathrm{I}^{-}$, is to be evaluated

Variables changed:

XINT3 - the value of the integral $\mathrm{I}^{+}$or $\mathrm{I}^{-}$

XUP1 - variable containing values of upper limits of the integrals, one of the values XUP1 $=R_{0}+x_{S} \pm \sqrt{\rho^{2}-z_{B}^{2}}$ according to the value of $\mathrm{NZ}(=1,2)$. 
Significant internal variables:

$\mathrm{X}$ - array of successive values of $\zeta_{j}$ used to compute the value of the function $f\left(\zeta_{i}\right)$

FX - array of values of the integrands

$$
\left.I_{B}(\tilde{r}) \frac{d N_{f}}{d R}\right|_{R=R_{O+x_{s}} \pm \sqrt{\rho^{2}-z_{B}^{2}}}
$$

TXY - value of upper limit of integration

$\mathrm{PXY}$ - value of lower limit of integration 
SUBR $\emptyset U T I N E$ W3XYZ (ZRH $\emptyset, Z B, A C R B, W X Y Z)$

Subroutine W3XYZ calculates the number of fast ions produced per unit radius $\mathrm{dN}_{\mathrm{f}} / \mathrm{dR}$ at the four points where the pencil beam passes through a given flux surface. The geometry upon which the ordering of the computations is based is shown in Fig. 11. The outer points $P_{1}$ and $\mathrm{P}_{4}$ are treated separately from the inner points $\mathrm{p}_{2}$ and $\mathrm{p}_{3}$. The switch variable NZ distinguishes between these two cases. Values of $\mathrm{dN}_{\mathrm{f}} / \mathrm{dR}$ for points $p_{1}$ and $p_{4}$ are computed when $\mathrm{NZ}=1$ and for points $\mathrm{p}_{2}$ and $\mathrm{p}_{3}$ when $\mathrm{NZ}=2$.

The general equation upon which this subroutine is based is ${ }^{2}$

$$
\begin{aligned}
I_{B}(\tilde{r}) \frac{d N_{f}}{d R}=I_{B}(\tilde{r}) \frac{\tilde{n}(r)}{\tilde{n}(0)} \frac{R}{\sqrt{R^{2}-R_{B}^{2}}} \int \exp \left[\frac{1}{\tilde{\lambda}} \int_{R_{0}+\sqrt{a^{2}-z_{B}^{2}}}^{R}\right. \\
\left.\left.+\int_{R}^{R_{B}}-\int_{R_{B}}^{R_{0}}+\sqrt{a^{2}-z_{B}^{2}}\right] \frac{\tilde{n}(r)}{\tilde{n}(0)} \frac{R^{\prime} d R^{\prime}}{\sqrt{R^{\prime 2}-R_{B}^{2}}}\right)
\end{aligned}
$$

where $r$ is the radial distance from the torus minor axis to the pencil beam axis, $\tilde{r}$ the radial distance from the center of the injected neutral beam to the pencil beam axis and $\tilde{\lambda}_{0}=\tilde{\lambda}_{c x}(0)$ the charge-exchange mean free path (the beam absorption mean free path).

\section{Called from: $\quad$ RPT $3 X Y$}

Subroutines called: GAUSS8, YTERP

Commons required: $\quad \mathrm{CN}, \mathrm{D}, \mathrm{E}, \mathrm{HZ}$, INTRP

Variables required:

$$
\begin{aligned}
& \text { ARH } \varnothing \text { - distance from the stagnation point } x_{s} \text { to points in } \\
& \text { the plasma, i.e., radius of a flux surface } \\
& \mathrm{ZB} \text { - vertical displacement } \mathrm{z}_{\mathrm{B}} \text { of pencil beam from plane } \\
& \text { of symmetry of the torus, cm } \\
& A C R B \text { - an array of current values of } R_{B} \text { (there are nested } \\
& \text { integrals) } \\
& \text { NP - number of points used by the Gauss-Chebyshev } \\
& \text { quadrature formula } \\
& \text { A - torus minor radius, } \mathrm{cm}
\end{aligned}
$$


ORNL-DWG 76-2313

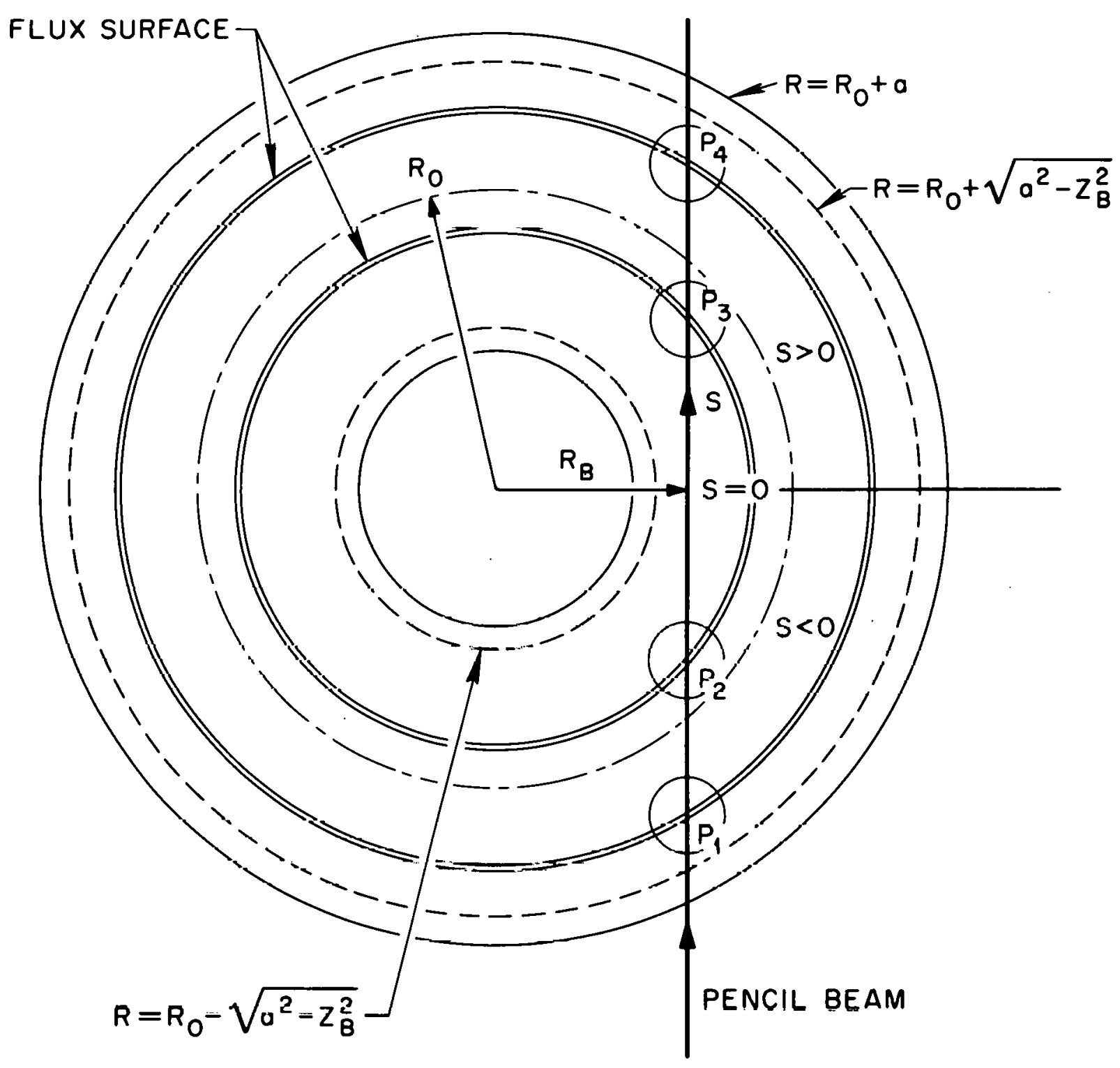

Fig. 11. Pencil beam geometry for neutral beam injection. 
RC - radius of center of injected neutral beam measured from torus vertical axis, $\mathrm{cm}$

RO - major radius of the torus, $\mathrm{cm}$

$\mathrm{NZ} \quad-$ switch used to determine at which pair of points to compute $\mathrm{dN}_{\mathrm{f}} / \mathrm{dR}$

$=1, \mathrm{p}_{1}$ and $\mathrm{P}_{4}$

$=2, \mathrm{p}_{2}$ and $\mathrm{p}_{3}$

XUP1 - a variable containing one of the limits for the integrals, with one of the values XUP1 $=R_{0}+x_{S}$ $\pm \sqrt{p^{2}-z_{B}^{2}}$ according to the value of $N Z(=1,2)$

BEAM - square of the half width of the assumed Gaussian profile shape of the injected neutral beam

DXX - spacing interval for the XXX array

XXX - independent variable array containing values of radius for which the plasma density is known

PIDEN - array containing plasma density profile normalized to the central density (see description of subroutine SUBH)

$\frac{\text { Variables changed: }}{\text { WXYZ - array of values of the quantity }\left.\frac{d N_{f}}{d R}\right|_{R}=R_{o}+x_{s} \pm \sqrt{\rho^{2}-z_{B}^{2}}}$

CRB - current value of $R_{B}$, required by a nested integral

$\mathrm{RH} \emptyset$ - value of radius of a particular flux surface, cm

$X Z B$ - a particular value of $z_{B}$, used in a nested integral, cm Significant internal variables:

A1 - attenuation factor for beam from entrance point of plasina tu $\dot{P}_{1}$

A2 - attenuation factor for beam from entrance point of plasma to $\mathrm{p}_{4}$

A3 - attenuation factor for beam from entrance point of plasma to $\mathrm{p}_{2}$

A4 - attenuation factor for beam from entrance point of plasma to $\mathrm{p}_{3}$

A7 - value of neutral beam current profile $I_{B}(\tilde{r})$ at the location of the pencil beam

$\mathrm{XL}, \mathrm{XL1}$ - values of lower limits of integration

XU, XUP1- values of upper limits of integration 
SUBR ØUTINE GAUSS8(A, B, ANS)

Subroutine GAUSS8 uses a 12-point Gauss-Chebyshev quadrature formula to evaluate integrals of the form

$$
\text { ANS }=\frac{1}{\tilde{\lambda}_{0}} \int_{A}^{B} \frac{\tilde{n}(r) R^{\prime} d R^{\prime}}{\sqrt{R^{\prime 2}-R_{B}^{2}}}, r=R^{\prime}-R_{0}
$$

which appear in the general form of the absorption decrement ${ }^{2}$

$$
\text { I) }(s)=\int_{-\infty}^{s} \frac{d s^{\prime}}{\lambda\left(s^{\prime}\right)}
$$

for a pencil beam component of an injected neutral beam. Let $R_{B}=R^{\prime} \xi$; then

$$
\text { ANS }=\frac{1}{\tilde{\lambda}_{0}} \int_{R_{e} / A}^{R_{B} / B} \tilde{n}\left(\frac{R_{B}}{\xi}-R_{0}\right)\left(-\frac{R_{B}}{\xi^{2}}\right) \frac{d \xi}{\sqrt{1-\xi^{2}}} \text {. }
$$

This integral is of the form $\int_{a}^{b} f(x) d x$ and must be modified as follows to obtain the form required for the Gauss-Chebyshev quadrature:

$$
\int_{a}^{b} w(x) \frac{f(x)}{w(x)} d x=\int_{a}^{b} \frac{F(x)}{\sqrt{1-x^{2}}} d x
$$
where $w(x)=\frac{1}{\sqrt{1-x^{2}}}$ is the weight function. The Gauss-Chebyshev formula
is $^{3}$

$$
\int_{-1}^{1} \frac{F(z)}{\sqrt{1-z^{2}}} d z \doteq \frac{\pi}{n} \sum_{j=1}^{n} F^{\prime}\left(z_{j}\right)
$$

where for the integral to be evaluated,

$$
F\left(z_{j}\right)=\sqrt{1-z_{j}^{2}} f\left(\frac{a+b}{2}+z_{j} \frac{b-a}{2}\right)=\sqrt{1-z_{j}^{2}} f\left(\zeta_{j}\right)
$$


with

$$
f\left(\zeta_{j}\right)=\tilde{n}\left(\frac{R_{B}}{\zeta_{j}}-R_{o}\right)\left(-\frac{R_{B}}{\zeta_{j}}\right) \frac{1}{\sqrt{1-\zeta_{j}^{2}}} .
$$

The $z_{j}$ are the zeros of the Chebyshev polynominals of degree $n$.

Values for the density (normalized to the plasma central density)

$\tilde{n}(r)$ at the radii corresponding to the $z_{j}$ are computed using a linear interpolation of the normalized density profile array.

Called from: W3XYZ

Commons required: $\mathrm{CN}, \mathrm{D}, \mathrm{E}, \mathrm{HZ}$, INTRP

Variables required:

$$
\begin{aligned}
& \text { A - lower limit of the integral } \\
& \mathrm{B} \text { - upper limit of the integral } \\
& \mathrm{CRB} \text { - current value of } \mathrm{R}_{\mathrm{B}} \text { (there are nested integrals), the } \\
& \text { distance from the major axis of the torus to the } \\
& \text { point of tangency of the pencil beam } \\
& \text { NP - the number of points used by the quadrature formula } \\
& \mathrm{S} \quad-\begin{array}{l}
\text { array of zeros } \mathrm{z}_{j} \text { of the Chebyshev polynominals of } \\
\text { degree NP }
\end{array} \\
& \text { RO - torus major radius, } \mathrm{cm} \\
& \text { ZB - pencil beam vertical displacement } z_{B} \text { from plane of } \\
& \text { symmetry of the torus, } \mathrm{cm} \\
& \mathrm{AB} \text { - torus minor radius, } \mathrm{cm} \\
& \text { DXX - spacing interval for independent variable array } \\
& \text { corresponding to the normalized density profile } \\
& \text { PIDEN - plasma density profile array, normalized to the } \\
& \text { central density (see description of subroutine SUBH) } \\
& \text { XXX - independent variable array corresponding to the } \\
& \text { normalized density profile }
\end{aligned}
$$

Variables changed:

ANS - the value of the integral

Significant internal variables:

$\mathrm{X}$ - the variable which takes on the successive values of $\zeta_{j}$ used to compute the value of the function $f\left(\zeta_{j}\right)$ 
SUBRøUTINE GAUSST

Subroutine GAUSST does the integration over $\theta$ for $H(r, \theta)$ to get the fast ion production profile $\langle\mathrm{H}(\mathrm{r}, \theta)\rangle_{\theta}$ according to

$$
H(r)=\langle H(r, \theta)\rangle_{\theta}=\frac{1}{\pi} \int_{0}^{\pi} H[r(\theta)] d \theta \text {, }
$$

using an eight point Gaussian quadrature. Figure 11 shows the geometry and conventions used as the basis for the computational procedure.

Called from: BUBH

Function called: YTERP

Commons required: $\quad$ D, TA

Variables required:

NGLP1 - number of computational grid elements plus one used for the $\mathrm{H}[\rho(\theta)]$ profile

XSS - displacement between the magnetic axis and the stagnation point (see Fig. 10), cm

RH $\emptyset \mathrm{MAX}$ - maximum value of $\mathrm{RH} \emptyset$ permitted, as determined by the position of the torus wall or diverter relative to the stagnation point

DELN - AM/NCGLM, radial increment with respect to torus minor radius $\mathrm{AM}$, $\mathrm{cm}$

DELII -..- RHøMAX/NCGLM, radial increment with respect to maximum value of $\mathrm{RH} \phi, \mathrm{cm}$

RV - array of radial values at which the fast ion production profile, $H(r, \theta)$ before angle averaging, is determined, i.e., $0 \leqslant \mathrm{RV}(\mathrm{I})<\mathrm{RH} \emptyset \mathrm{MAX}$

HRH $\varnothing$ - array of values of $H(r, \theta)$

Variablea changed:

$\mathrm{RH} \emptyset$ - distance from the stagnation point to points in the plasma detined by RHO $\leqslant$ AM - XSS, where AM is the Lurue millor ralius

HTT - value of the integral $\langle\mathrm{H}(x, \theta)\rangle_{\theta}$ at each of the points in the interval [0,AM] with grid spacing DELN. 
FUNCTI $\emptyset \mathrm{N}$ XTERP (XVAL, X, F, NøPTS, T $\varnothing \mathrm{L}, \mathrm{NCALL})$

Function XTERP returns a value interpolated from the F-array corresponding to a value XVAL of the independent variable when XVAL lies in the range of the X-array values. The routine uses a Newton backward difference-forward difference method to get the interpolated value. The number of iterations is fixed at four since the F-array values do not necessarily lie along a smooth curve. High order polynomial interpolations to the F-array values generally cause numerical problems.

Called from: BEAM (alternate ENTRY INJECT), SUBH

Variables required:

XVAL - value of independent variable for which an interpolated value of the function (dependent variable) is desired

$\mathrm{X}-$ table of values of independent variable

$F \quad-$ cable of values of dependent variable

NØPTS - number of points for which function values exist in the $\mathrm{F}$-array

TøL - tolerance factor for test of error size

NCALL - call number of the subroutine, used only for printing out diagnostics

Variable changed:

XTERP - interpolated value of the dependent variable

\section{Limitations:}

The error test value is given by $|\mathrm{T} \emptyset \mathrm{L} * \mathrm{~F}(1)|$ 
FUNCTIØN YTERP (RRR, XXX, PIDEN, DXX, NN)

Function YTERP returns the value of a tabulated function at some specified point using a linear interpolation procedure. The routine assumes the values in the independent variable array are equally spaced. Let $f_{i}=f\left(x_{i}\right)$ and $\Delta=x_{1+1}-x_{i}$; then the interpolation formula is

$$
f(x)=f_{i}+\left[f_{i+1}-f_{i}\right]\left(x-x_{i}\right) / \Delta
$$

If $a$ value of $x$ outside the range of the tables of values for $f_{i}$ and $x_{i}$ is supplied, the value of the function returned is an extrapolation based on the two end values of the tables appropriate for the cases when $\mathrm{x}<\mathrm{x}_{1}$ and when $\mathrm{x}>\mathrm{x}_{\mathrm{NN}}$. The tables are assumed to be ordered according to increasing values of $x_{i}$.

\section{Called from: BEAM (alternate ENTRY INJECT)}

Variables required:

RRR - value of independent variable for which an intexpolated value of the function (dependent variable) is desired

$\mathrm{xxX}$ - table of equally spaced values of independent varlable, $x_{i}$ PIDEN - table of values of dependent variable, $f_{i}$ DXX - independent variable"spacing, $\Delta=\mathrm{x}_{i+1}-\mathrm{x}_{\mathbf{i}}$

NN - the number of pairs of values $\left(f_{i}, x_{i}\right.$ ) in the dependent
and independent variable tables

Variable changed:

YTERP $=f(x)$ 


\section{SUBR $\emptyset U T I N E$ FPMØME}

Subroutine FPM $\varnothing$ ME is the main calling routine for evaluating the moments of the Fokker-Planck equation, which is used to describe the slowing down of the injected neutral beam in the toroidal plasma. The moments are evaluated by a series of 30 function subprograms. Figure 12 shows the function routines and order of calls used to do the moments calculation. The expressions for the moments were derived by J. D. Callen. ${ }^{4}$ The variables described below are used throughout the function subprograms, so are defined here only to avoid unnecessary repetition.

The velocity of the neutral beam at the point of entrance into the plasma is given by

$$
\mathrm{VO}=\mathrm{v}_{\circ} \sqrt{\frac{2 \mathrm{EO} \emptyset}{\mathrm{m}_{\mathrm{f}}}} \quad, \mathrm{cm} / \mathrm{sec}
$$

where

$$
\begin{aligned}
& \mathrm{EO} \emptyset=\mathrm{E}_{\mathrm{o}}=\text { the energy of the injected neutral beam component, } \mathrm{eV} \\
& \text { and } \mathrm{m}_{\mathrm{f}}=\text { the mass of a neutral beam particle, amu. }
\end{aligned}
$$

The energy $\mathrm{E}_{c}$, above which the injected neutral beam slowing is due to the plasma electrons and below which slowing is due to plasma ions, is given by

$$
\operatorname{EC} \emptyset=E_{c}=14.8 \mathrm{~T}_{\mathrm{e}}\left(\mathrm{m}_{\mathrm{f}} / \mathrm{m}_{\mathrm{H}}\right)^{1 / 3}\left(\mathrm{~m}_{\mathrm{f}} / \mathrm{m}_{\mathrm{i}}\right)^{2 / 3}[\mathrm{z}]^{2 / 3}, \mathrm{eV}
$$

where

$$
\begin{aligned}
& \mathrm{m}_{i}=\text { mass of the plasma ions, amu } \\
& \mathrm{m}_{\mathrm{H}}=\text { mass of the proton, amu } \\
& \mathrm{T}_{\mathrm{e}}=\text { electron temperature, } \mathrm{eV} \text { and } \\
& {[\mathrm{Z}]=\left(1 / \mathrm{n}_{\mathrm{e}}\right)\left\{\sum_{\text {specles, } \mathrm{k}}\left(\mathrm{m}_{\mathrm{H}} / \mathrm{m}_{\mathrm{k}}\right) \mathrm{n}_{\mathrm{k}} \mathrm{z}_{\mathrm{k}}{ }^{2}\right\} /\left\{\sum_{\text {species, }} \sum_{k} \mathrm{n}_{k}\right\} .}
\end{aligned}
$$

The velocity $v_{c}$ corresponding to $\mathrm{E}_{c}$ is given by

$$
\operatorname{vc} \phi=v_{c}=\sqrt{\frac{2 E_{c}}{m_{f}}}, \mathrm{~cm} / \mathrm{sec} .
$$




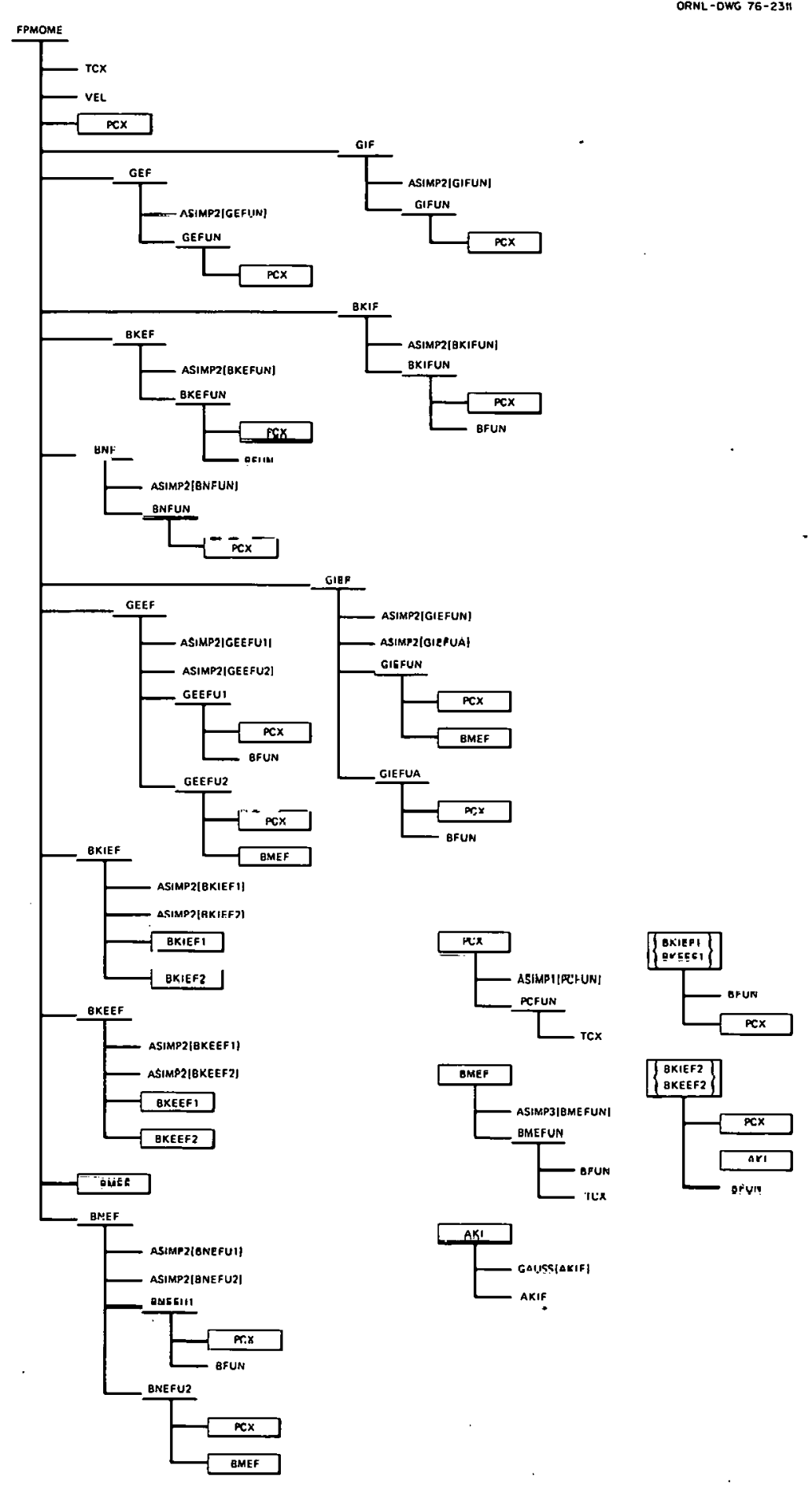

Fig. 12. Diagram of function routines and their order of call for the calculation of the moments of the Fokker-Planck equation. 
The neutral beam slowing down time $\tau_{s}$ is given by

$$
\operatorname{TS} \emptyset=, \tau_{s}=0.12\left(\mathrm{~T}_{\mathrm{e}} / 10^{3} \mathrm{eV}\right)^{3 / 2}\left(\mathrm{~m}_{\mathrm{f}} / \mathrm{m}_{\mathrm{H}}\right) /\left[\left(\mathrm{n}_{\mathrm{e}} / 10^{13}\right) \mathrm{z}_{\mathrm{f}}^{2}\right], \text { sec }
$$

where $\mathrm{n}_{e}$ is the plasma electron density, in units of $10^{-13} \mathrm{~cm}^{-3}$.

The time interval $\tau_{f}$, used as one of the criteria to determine when the Fokker-Planck $\operatorname{code}^{4}$ solution has reached a steady state condition, is given by

$$
T F \emptyset=\tau_{F}=\frac{\tau}{3} \ln \left[1+\left(v_{o} / v_{c}\right)^{3}\right]
$$

At time $t$ following entrance into the plasma, a fast ion generated from the injected neutral beam has slowed to a velocity $v(t)$ and corresponding energy $E(t)$ given by

$$
\operatorname{ET} \emptyset=E(t)=\frac{1}{2} m_{f} v^{2}(t), e V \cdot
$$

The remaining variables are described in detail under the headings of the varlous function subprograms called by subroutine FPM $\varnothing$ ME.

Called from: BEAM (alternate ENTRY INJECT)

Furictions called: BKEEF, BKEF, BKIEF, BKIF, BMEF, BNEF, BNF, GEEF, GEF, GIEF, GIF, PCX, TCX, VEL

Commons required: INPUT, ØUTPUT (see Appendix II)

Variables changed: $E O \emptyset, E C \emptyset, E T \emptyset, T \varnothing, T S \emptyset, T F \emptyset, \operatorname{VO} \emptyset, \operatorname{VC} \emptyset, \operatorname{VT} \emptyset$

$$
\begin{aligned}
& \operatorname{TCXV} \emptyset-\tau_{c x}(v) \text {, the mean lifetime of a neutral beam particle before } \\
& \text { undergoing a charge-exchange event with a plasma ion } \\
& \text { GE(GI) - fraction of injected neutral beam power transferred } \\
& \text { to the plasma electrons (ions) } \\
& \mathrm{BKE}(\mathrm{BKI}) \text { - fraction of fast ion momentum transferred to the } \\
& \text { plasma electrons (ions) } \\
& \text { BN - number of stored fast ions resulting from the injected } \\
& \text { neutral beam } \\
& \text { PCXVT - probability that a charge-exchange event will not occur } \\
& \text { Q during the time a neutral beam particle slows from the } \\
& \text { injection velocity } v_{0} \text { to the velocity } v(t) \\
& \text { GEE(GIE) - correction to the injected neutral beam power transferred } \\
& \text { to the plasma electrons (ions) due to the electric field } \\
& \text { BKEE - correction to momentum transferred from injected neutral } \\
& \text { (BKIE) beam to the plasma electrons (ions) due to the electric } \\
& \text { field }
\end{aligned}
$$


BME - an integral arising from one of the moments of the Fokker-Planck equation and related to the electric field correction

$B N E$ - correction to the number of stored fast ions due to the electric field 
FUNCTIØN VEL (TIME)

Function VEL computes the velocity of the injected neutral beam at a point along the beam path reached by the beam particles at a time TIME following initial entrance into the plasma and taking into account the slowing down process. The expression used is

$$
v(t)=\left\{\begin{array}{l}
{\left[v_{o}{ }^{3} \exp \left\{-3 t / \tau_{s}\right\}-v_{c}{ }^{3}\left(1-\exp \left\{-3 t / \tau_{s}\right\}\right)\right]^{1 / 3}, t<\tau_{F}} \\
\sqrt{\frac{2 E_{i o n}}{M_{\text {ion }}}}, t>\tau_{F}, \text { or } t>10^{6} \text { if } \tau_{F}>10^{6}
\end{array}\right.
$$

where

$$
\begin{aligned}
\tau_{F}= & \frac{\tau_{s}}{3} \ln \left[1+v_{0}^{3 / v_{c}}\right], \\
v_{0} \equiv & \text { velocity of neutral beam at point of entrance into the plasma, and } \\
v_{c} \equiv & \begin{array}{l}
\text { a critical velocity, above which the neutral beam slowing is due } \\
\text { to the plasma electrons and below which slowing is due to plasma } \\
\text { ions. }
\end{array}
\end{aligned}
$$

Called from: $\quad$ FPM $\emptyset \mathrm{ME}$

Commons required: INPUT, ØUTPUT (see Appendix II)

Variable required: TIME

Variables changed: $\quad$ VEL $=v(t)$ 
FUNCTI $\emptyset \mathrm{N}$ TCX $(\mathrm{V})$

Function TCX returns the value of the mean lifetime (in seconds) of a neutral beam particle before undergoing a charge-exchange collision with a plasma ion. The expression for this lifetime is

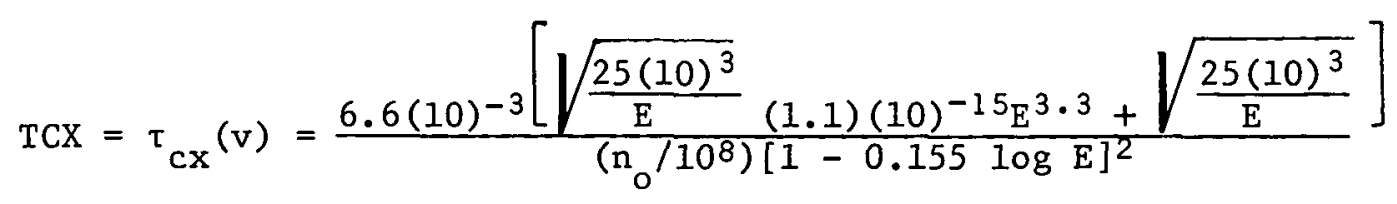

where

$$
\mathrm{E}=\frac{1}{2} \mathrm{~m}_{\mathrm{H}} \mathrm{v}^{2}
$$

Called from: $\quad$ FPM $\varnothing \mathrm{ME}$, BMEFUN

Common required: INPUT (see Appendix II)

Variable required: $\mathrm{V}=\mathrm{v}$

Variable changed: TCX

Significant internal variable:

DNO $=\mathrm{n}_{0} / 10^{8}$ where $\mathrm{n}_{0}$ is the plasma neutral density, $\mathrm{cm}^{-3}$ 
FUNCTI $\varnothing \mathrm{N} P C X(\mathrm{~V}$, ICTCX)

Function PCX gives the probability of a charge-exchange event not occurring between an injected neutral beam particle and the plasma as the neutral beam particle slows down. This probability is given by

$$
\mathrm{PCX} \equiv \mathrm{p}_{\mathrm{CX}} \mathrm{v}(\mathrm{t})=\exp \left\{-\tau_{\mathrm{s}} \int_{\mathrm{v}(\mathrm{t})}^{\mathrm{v}} \mathrm{o} \frac{\mathrm{v}^{\prime 2} \mathrm{dv}^{\prime}}{\mathrm{v}^{\prime 3}+\mathrm{v}_{\mathrm{c}}{ }^{3}} \frac{1}{\tau_{\mathrm{cx}}\left(\mathrm{v}^{\prime}\right)}\right\}
$$

where

$$
\begin{aligned}
& v(t) \quad-\text { velocity to which neutral beam has slowed after time } t \\
& \text { following entrance into the plasma, } \\
& \tau_{s} \quad-\text { injected neutral beam slowing down time, } \\
& \begin{aligned}
\tau_{c x}\left(v^{\prime}\right)- & \text { mean lifetime of a neutral beam particle before under- } \\
& \text { going charge exchange with a plasma ion, }
\end{aligned} \\
& \text { vo - neutral beam injection velocity, } \\
& v_{c} \quad-\text { critical velocity, above which the neutral beam slowing } \\
& \text { is due to the plasma electrons and below which slowing } \\
& \text { is due to plasma ions. }
\end{aligned}
$$

Called from: $\quad$ FPM $\emptyset \mathrm{ME}$, GIFUN, GEFUN, BKIFUN, BKEFUN, BNFUN, GIEFUN, GIEFUA, GEEFU1, GEEFU2, BKIEF1, BKIEF2, BKEEF1, BKEEF2, BNEFU1, BNEFU2

Subroutines called: ASIMP1, PCFUN (through an EXTERNAL reference)

Commons required: INPUT, ØUTPUT (see Appendix II)

Variables required: $\quad V=v(t)$

$$
\begin{aligned}
\text { ICTCX }- & \text { switch used to choose expression to be evaluated for } \\
& \text { value of PCX. If } \tau_{r x} \text { is constant }(\text { ICTCX } \neq 0) \text {, then } \\
& \text { the approximate relation used is } \\
\mathrm{P}_{C X}(v) \simeq & {\left[\left(v_{o}^{3}+v_{c}^{3}\right) /\left(v^{3}+v_{c}^{3}\right)\right]^{-\tau_{s} /\left(3 \tau_{c x}\right)} }
\end{aligned}
$$

Variable changed: PCX 
FUNCTIØN PCFUN (Y)

Function PCFUN returns the value of the integrand

$$
\text { PCFUN }=-\frac{\tau_{s}}{\tau_{c X}\left(v^{\prime}\right)} \frac{\left(v^{\prime} / v_{o}\right)^{2}}{\left(v^{\prime} / v_{o}\right)^{3}+\left(v_{c} / v_{o}\right)^{3}}
$$

used by the adaptive Simpson's rule quadrature subroutine called by function PCX. The variables are defined in the description of function PCX.

Called from: ' ASIMPI, reterred to through EXTERNAT statement in function PCX

Commons required: INPUT, ØUTPUT (see Appendix IT)

Variable required: $\mathrm{Y}=\mathrm{v}^{\mathrm{r}} / \mathrm{v}_{\mathrm{O}}$

Variable changed: PCFUN 
FUNCTI $\emptyset \mathrm{N}$ GIF (VT)

Function GIF calculates the amount of injected neutral beam power transferred to the plasma ions according to the equation

$$
G I F=G_{i}=\frac{2}{v_{0}^{2}} \int_{v(t)}^{v_{0}} v d v \frac{v_{c}^{3}}{v^{3}+v_{c}{ }^{3}} p_{c x}(v)
$$

where

$$
\begin{aligned}
& v_{c} \text { - a critical velocity, above which the neutral beam slowing is } \\
& \text { due to the plasma electrons and below which slowing is due } \\
& \text { to plasma ions, } \\
& v_{0} \text { - velocity of the neutral beam at point of entrance into the } \\
& \text { plasma, } \\
& \mathrm{v}(\mathrm{t}) \quad \text { - velocity of the injected neutral beam at a point along the } \\
& \text { beam path reached at time } t \text { following initial entrance into } \\
& \text { the plasma, } \\
& \mathrm{p}_{\mathrm{cx}}(\mathrm{v}) \text { - probability of avoiding a charge-exchange event by the } \\
& \mathrm{v} \quad \text { neutral beam as it slows down, }
\end{aligned}
$$

Called from: $\quad$ FPMфME

Subroutines called: ASIMP2, GIFUN (through an EXTERNAL reference)

Commons required: INPUT, ØUTPUT (see Appendix II)

Variable required: $\quad \mathrm{VT}=\mathrm{v}(\mathrm{t})$

Variable changed: GIF 
FUNCTIØN GIFUN (Y)

Function GIFUN calculates the value of the integrand for the integral calculated by function GIF. The expression for the integrand is referred to by the two-point Simpson's rule quadrature subroutine called by function GIF. The integrand expression whose value is computed is

$$
\text { GIFUN }=\frac{2 p_{c x}(v)\left(v / v_{o}\right)\left(v_{c} / v_{o}\right)^{3}}{\left[\left(v / v_{0}\right)^{3}+\left(v_{c} / v_{0}\right)^{3}\right]} .
$$

Called from: ASIMF 2, referred to througlı EXTERINAL statement in GIF

Function called: . PCX

Commons required: INPUT, ØUTPUT (see Appendix II)

Variable required: $\quad \mathrm{Y}=\mathrm{v} / \mathrm{v}_{\mathrm{o}}$

Variable changed: GIFUN 
FUNCTI $\emptyset \mathrm{N}$ GEF (VT)

Function GEF calculates the amount of neutral beam power transferred to the plasma electrons according to the equation

$$
G E F=G_{e}=\frac{2}{v_{o}^{2}} \int_{v(t)}^{v_{0}} v d v \frac{v^{3}}{v^{3}+v_{c}^{3}} p_{c x}(v)
$$

where

$$
\begin{aligned}
& v_{c} \quad \text { a critical velocity, above which the neutral beam slowing } \\
& \text { is due to the plasma electrons and below which slowing is } \\
& \text { due to plasma ions, } \\
& v_{0} \text { - velocity of the neutral beam at point of entrance into } \\
& \text { the plasma, } \\
& \mathrm{v}(\mathrm{t}) \quad \text { velocity of the injected neutral beam at time } t \text { following } \\
& \quad \text { initial entrance into the plasma, } \\
& \mathrm{p}_{\mathrm{cx}}(\mathrm{v}) \text { - probability that a charge-exchange event does not occur } \\
& \mathrm{v} \quad \text { as the neutral beam slows down in the plasma, } \\
& \mathrm{v} \quad \text { velocity of the neutral beam. }
\end{aligned}
$$

Called from: $\quad$ FPMфME

Subroutines called: ASIMP2, GEFUN (through an EXTERNAL reference)

Commons required: INPUT, ØUTPUT (see Appendix II)

Variable required: $\quad \mathrm{VT}=\mathrm{v}(\mathrm{t})$

Variable changed: GEF 
FUNCTI $\emptyset N$ GEFUN (Y)

Function GEFUN calculates the value of the integrand for the integral calculated by function GEF. The expression for the integrand is referred to by the two-point Simpson's rule quadrature subroutine called by the function GEF. The integrand expression whose value is computed is

$$
\text { GEFUN }=\frac{.2 \mathrm{P}_{\mathrm{cx}}(\mathrm{v})\left(\mathrm{v} / \mathrm{v}_{\mathrm{o}}\right)^{4}}{\left[\left(\mathrm{v} / \mathrm{v}_{\mathrm{o}}\right)^{3}+\left(\mathrm{v}_{\mathrm{c}} / \mathrm{v}_{\mathrm{o}}\right)^{3}\right]}
$$

Cialled from: ASIMP2, Leferied to through EXTERNAL statement in GEF Subroutine called: HLX

Commons required: INPUT, ØUTPUT (see Appendix II)

Variables required: $\mathrm{Y}=\mathrm{v} / \mathrm{v}_{\mathrm{O}}$

Variable changed: GEFUN 
FUNCTI $\emptyset \mathrm{N}$ BKIF (VT)

Function BKIF computes the amount of momentum transferred from the injected neutral beam to the plasma ions according to the equation

$$
B K I F=k_{i}=\frac{1}{v_{0}} \int_{v(t)}^{v_{0}} d v \frac{v_{c}^{3}}{v^{3}+v_{c}^{3}}\left[1+\frac{m_{i}<Z>}{m_{f}[z]}\right] p_{c x}(v) b(v)
$$

where

$$
\begin{aligned}
& b(v)=\left[\left(\frac{v_{0}^{3}+v_{c}^{3}}{v^{3}+v_{c}{ }^{3}}\right)_{v_{0}^{3}}^{v^{3}}\right]^{\frac{m_{i}<Z>}{3 m_{f}[z]}}, \\
& \left\langle Z>=\underset{k}{\left[\sum n_{k} z_{k}^{2}\right] / \underset{k}{\left[\sum n_{k} z_{k}\right],}},\right.
\end{aligned}
$$

and

$$
\left.\left.[z]=\underset{k}{\left[\sum\right.} \frac{m_{\text {proton }}}{m_{k}} n_{k} z_{k}{ }^{2}\right] / \underset{k}{\left[\sum\right.} n_{k} z_{k}\right] \text {. }
$$

The remaining variables have been defined in the descriptions of FPM $\varnothing \mathrm{ME}$ and of the first few functions called by FPMØME.

Called from: $\quad$ FPMØME

Subroutines called: ASIMP2, BKIFUN (through an EXTERNAL reference)

Cummuns required: INPUT',

Variable required: $\quad V T=v(t)$

Variable changed: $\quad B K I F$ 
FUNCTI $\emptyset \mathrm{N}$ BKIFUN (Y)

Function BKIFUN returns the value of the integrand for the integral calculated by function BKIF. The expression for the integrand is referred to by the two-point Simpson's rule quadrature subroutine called by function $\mathrm{BKIF}$. The integrand expression whose value is computed is

$$
\text { BKIFUN }=\frac{\left(v_{c} / v_{o}\right)^{3}}{\left(v / v_{n}\right)^{3}+\left(v_{c} / v_{o}\right)^{3}} p_{c x}(v)\left[1+\frac{m_{i}<Z>}{m_{f}[z]}\right]\left[\left(\frac{v_{o}^{3}+v_{c}{ }^{3}}{v^{3}+v_{e}^{3}}\right)^{3} v_{o}^{3}\right]^{\frac{m_{i}<Z>}{3 m_{f}[z]}} .
$$

Called from: ASIMH2, referired to chrough EXTERNAL statement in BKIF

Functions called: PCX, BFUN

Commons required: INPUT, ØUTPUT (see Appendix II)

Variable required: $\quad \mathrm{Y}=\mathrm{v} / \mathrm{v}_{\mathrm{O}}$

Variable changed: BKIFUN 
FUNCTI $\emptyset N$ BFUN (V)

Function BFUN returns the value of the expression

$$
\text { BFUN }=b(v)=\left[\frac{v_{0}{ }^{3}+v_{c}{ }^{3}}{v^{3}+v_{c}{ }^{3}}\left(\frac{v}{v_{0}}\right)^{3}\right]^{\frac{m_{i}<Z>}{3 m_{f}[z]}} .
$$

Called from: $\quad$ BKIF, BKEF, GIEFUA, GEEFU1, BKIEF1, BKIEF2, BKEEF1, BKEEF 2, BNEFU1

Commons required: INPUT, ØUTPUT (see Appendix II)

Variable required:

$\mathrm{V}$ - the velocity $\mathrm{v}$ of the neutral beam at a specified time following injection into the plasma

Variable changed: BFUN 
FUNCTI $\emptyset \mathrm{N}$ BKEF (VT)

Function BKEF computes the amount of momentum transferred from the neutral beam to the plasma electrons according to the equation

$$
\text { BKEF }=K_{e}=\frac{1}{v_{0}} \int_{v(t)}^{v_{0}} d v \frac{v^{3}}{v^{3}+v_{c}{ }^{3}} p_{c x}(v) b(v)
$$

where

$$
\begin{aligned}
& b(v)=\left[\frac{v_{0}^{3}+v_{c}^{3}}{v^{3}+v_{c}^{3}}\left(\frac{v}{v_{0}}\right)\right]^{3} \frac{\frac{i}{3 m_{f}[z]}}{\frac{m_{i}<z}{3}},
\end{aligned}
$$

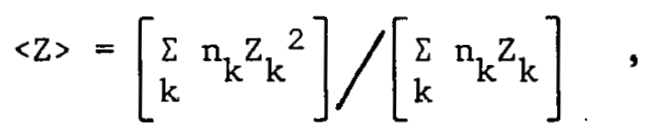

and

$$
[z]=\left[\sum \frac{m_{\text {proton }}}{m_{k}} n_{k} z_{k}\right] /\left[\begin{array}{ll}
\sum & n_{k} z_{k} \\
k &
\end{array}\right] \text {. }
$$

The remaining variables have been defined in the descriptions of FPMøME and of the first few functions called by FPM $\emptyset \mathrm{ME}$.

Called from:

FPM $\varnothing \mathrm{ME}$

Subroutines called: ASIMP2, BKEFUN (through an EXTERNAL reference)

Commons required: INPUT, ØUTPUT (see Appendix II)

Variable required: $\quad \mathrm{VT}=\mathrm{v}(\mathrm{t})$

Variable changed: $\quad$ BKEF 
FUNCTI $\emptyset N$ BKEFUN (Y)

Function BKEFUN returns the value of the integrand for the integral calculated by function BKEF. The expression for the integrand is referred to by the two-point Simpson's rule quadrature subroutine called by function BKEF. The integrand expression whose value is computed is

$$
\text { BEKFUN }=\frac{\left(v / v_{o}\right)^{3}}{\left(v / v_{0}\right)^{3}+\left(v_{c} / v_{o}\right)^{3}} \quad p_{c x}(v)\left[\frac{v_{0}^{3}+v_{c}^{3}}{v^{3}+v_{c}^{3}}\left(\frac{v}{v_{0}}\right)^{3}\right]^{\frac{m_{i}<Z>}{3 m_{f}[Z]}} \ldots
$$

Called from: $\quad$ ASIMP2, referred to through EXTERNAL statement in BKEF

Functions called: PCX, BFUN

Commons required: INPUT, ØUTPUT (see Appendix II)

Variable required: $Y=v / v_{0}$

Variable changed: BKEFUN 
FUNCTI $\emptyset \mathrm{N}$ BNF (VT, ICT)

Function BNF computes the number of stored fast ions according to

$$
B N F=N=\int_{v(t)}^{v_{0}} \frac{v^{2} d v}{v^{3}+v_{c}^{3}} p_{c x}(v)
$$

where all quantities have been defined in the descriptions of the first few functions called by FPMøME.

Called from: $\quad$ FPM $\emptyset \mathrm{ME}$

Subroutines called: ASIMP2, BNFUN (through an EXIEKNAL reference)

Commons required: INPUT, ØUTPUT (see Appendix II)

Variables required: $\quad \mathrm{V}^{\prime} \mathrm{I}=\mathrm{v}(\mathrm{t})$

ICT - switch used to choose expression to be evaluated for value of BNF. If $\tau_{\mathrm{CX}}$ is constant (ICT $\neq 0$ ), then the approximate relation used is

$N \simeq \frac{{ }^{\tau} c x}{\tau_{s}}\left[1-e^{-t / \tau_{c x}}\right]$ with $t= \begin{cases}t & t<\tau_{F} \\ \tau_{F}, & t>\tau_{F}\end{cases}$

(This feature has not yet been implemented.)

Variable changed: $\mathrm{BNF}$ 
FUNCTI $\emptyset \mathrm{N}$ BNFUN (Y)

Function BNFUN returns the value of the integrand for the integral calculated by function BNF. The expression for the integrand is referenced by the two-point Simpson's rule quadrature subroutine called by function BNF. The integrand expression whose value is computed is

$$
\text { BNFUN }=\frac{\mathrm{p}_{c x}(v)\left(v / v_{o}\right)^{2}}{\left(v / v_{0}\right)^{3}+\left(v_{c} / v_{o}\right)^{3}}
$$

Called from: ASIMP2, referred to through EXTERNAL statement in $\mathrm{BNF}$

Function called: PCX

Commons required: INPUT, ØUTPUT (see Appendix II)

Variable required: $Y=v / v_{0}$

Variable changed: BNFUN 
FUNCTI ØN GIEF (V)

Function GIEF calculates the correction to the amount of injected neutral beam power transferred to the plasma ions due to the electric field effects according to the equations

$$
\operatorname{GIEF}=G_{i}^{E}=\frac{2}{v_{0}^{2}} \int_{v(t)}^{v_{0}} v d v \frac{v_{c}^{3}}{v^{3}+v_{c}^{3}} p_{c x}(v)\left\{\frac{\xi_{0} v_{0} v^{2}}{v^{3}+v_{c}^{3}} b(v)-M^{E}(v)\right\}
$$

where

$$
\begin{aligned}
& M^{E}[v(t)]=B M E F=\xi_{0} v_{0} \int_{v(L)}^{v_{0}} \frac{v^{\prime 4} d v^{\prime}}{v^{\prime 3}+v_{\left.L^{3}\right)^{7}} \frac{{ }^{\prime} s}{\tau_{C A}\left(v^{\prime}\right)} b\left(v^{\prime}\right),} \\
& b(v)=B F U N=\left[\frac{v_{0}^{3}+v_{c}^{3}}{v^{3}+v_{c}^{3}}\left(\frac{v}{v_{0}}\right)^{3}\right]^{\frac{m_{i}<Z>}{3 m_{f}[Z]}}, \\
& \xi_{0}=\cos \theta_{0}=\text { the cosine of the neutral beam injection angle. }
\end{aligned}
$$

The remaining variables have been dellned in the descriptions of FPMøME and the first few functions called by FPMøME.

\section{Called from: $\quad$ 'PMOME}

Subroutines called: ASTMP2, GIEFUN (through an EXI'EKNAL reterence) GIEFUA (through an EXTERNAL reference)

Commons required: INPUT, ØUTPUT (see Appendix II)

Variable required: $\quad V=v(t)$

Variable changed: GIEF 
FUNCTI ØN GIEFUN (Y)

Function GIEFUN returns the value of the integrand for the second of two integrals contributing to the expression evaluated by GIEF. The expression for the integrand

$$
\text { GIEFUN }=2 \frac{v}{v_{0}} p_{c x}(v) M^{E}(v) \frac{\left(v_{c} / v_{o}\right)^{3}}{\left(v / v_{o}\right)^{3}+\left(v_{c} / v_{o}\right)^{3}}
$$

is referred to by the two-point Simpson's rule quadrature subroutine called by function GIEF.

Called from: $\quad$ ASIMP2, referred to through EXTERNAL statement in GIEF

Functions called: PCX, BMEF

Commons required: INPUT, ØUTPUT (see Appendix II)

Variable required: $Y=v / v_{0}$

Variable changed: GIEFUN 
FUNCTI $\emptyset \mathrm{N}$ BMEF (X)

Function BMEF returns the value of the expression

$$
\operatorname{BMEF}=M^{E}[v(t)]=\xi_{0} v_{0} \int_{v(t)}^{v_{0}} \frac{v^{\prime 4} d v^{\prime}}{\left(v^{\prime 3}+v_{c}^{3}\right)^{2}} \frac{\tau_{s}}{\tau_{c X}\left(v^{\prime}\right)} b\left(v^{\prime}\right) \text {. }
$$

Called from: $\quad$ GIEFUN, GEEFU2, BNEFU2

Subroutines called: ASIMP3, BMEFUN (through an EXTERNAL reference)

Commons required: INPUT, ØUTPUT (see Appendix II)

Variable required: $\quad X=v(t)$

Variable changed: $\quad B M E H$ 
FUNCTI $\emptyset$ BMEFUN (Y)

Function BMEFUN returns the value of the integrand for the integral calculated by function BMEF. The expression for the integrand

$$
\text { BMEFUN }=\frac{\left(v / v_{o}\right)^{4} \xi_{o} \tau_{s} b(v)}{\left[\left(v / v_{o}\right)^{3}+\left(v_{c} / v_{o}\right)^{3}\right]^{2} \tau_{c x}(v)}
$$

is referred to by the three-point Simpson's rule quadrature subroutine called by function BMEF.

Called from: ASIMP3, referred to through EXTERNAL statement in BMEF

Functions called: BFUN, TCX

Commons required: INPUT, ØUTPUT (see Appendix II)

Variable required: $Y=v / v_{0}$

Variable changed: BMEFUN 


\section{FUNCTI $\emptyset$ N GIEFUA (Y)}

Function GLEFUA returns the value of the integrand for the first of two integrals contributing to the expression evaluated by GIEF. The expression for the integrand

$$
\text { GIEFUA }=\frac{2 \xi_{o}\left(v / v_{o}\right)^{3}\left(v_{c} / v_{o}\right) 3 p_{c x}(v) b(v)}{\left[\left(v / v_{o}\right)^{3}+\left(v_{c} / v_{o}\right)^{3}\right]^{2}},
$$

where $\xi_{0}=\cos \theta_{0}$, the cosine of the neutral beam injection angle, is referred to by the two-point Simpson's rule quadrature subroutine called by function GIEF.

Called from: ASIMP2, referred to through EXTERNAL statement in GIEF Functions called: PCX, BFUN

Commons required: INPUT, ØUTPUT (see Appendix II)

Variable required: $\mathrm{Y}=\mathrm{v} / \mathrm{v}_{\mathrm{O}}$

Variable changed: GIEFUA 
FUNCTI $\emptyset \mathrm{N}$ GEEF (V)

Function GEEF calculates the correction to the amount of injected neutral beam power transferred to the plasma electrons due to the electric field effects according to the equations

$$
\operatorname{GEEF}=\frac{2}{v_{0}^{2}} \int_{v(t)}^{v_{0}} v d v \frac{v^{3}}{v^{3}+v_{c}^{3}} \cdot p_{c x}(v)\left\{\frac{\xi_{0} v_{0} v^{2}}{v^{3}+v_{c}^{3}} b(v)-M^{E}(v)\right\}
$$

where

$$
\begin{gathered}
M^{E}[v(t)]=B M E F=\xi_{0} v_{0} \int_{v(t)}^{v_{0}} \frac{v^{\prime 4} d v^{\prime}}{\left(v^{\left.\prime 3+v_{c} 3\right)^{2}} \frac{\tau_{s}}{\tau_{c x}\left(v^{\prime}\right)} b\left(v^{\prime}\right),\right.} \\
b(v)=B F U N=\left[\frac{v_{0}^{3}+v_{c}^{3}}{v^{3}+v_{c}^{3}}\left(\frac{v}{v_{0}}\right)^{3}\right]^{\frac{m_{i}<Z>}{3 m_{f}[Z]}},
\end{gathered}
$$

and $\xi_{0}=\cos \theta_{0}$, the cosine of the neutral beam injection angle. The remaining variables have been defined in the description of FPMØME and of the first few functions called by FPM $\phi \mathrm{ME}$.

Called from: $\quad$ FPM $\emptyset \mathrm{ME}$

Subroutines called: ASIMP2, GEEFU1 (through an EXTERNAL reference), GEEFU2 (through an EXTERNAL reference)

Cunmuns iequired: INPUT, ØUTPUT (see Appendix II)

Variable required: $\quad V=v(t)$

Variable changed: $\quad \mathrm{GEEF}$ 
FUNCTI

Function GEEFUl returns the value of the integrand for the first of two integrals contributing to the expression evaluated by GEEF. The expression for the integrand

$$
\text { GEEFU1 }=2 \xi_{o} p_{c x}(v) b(v)\left[\frac{\left(v / v_{o}\right)^{3}}{\left(v / v_{o}\right)^{3}+\left(v_{c} / v_{o}\right)^{3}}\right]^{2}
$$

where $\xi_{0}=\cos \theta_{0}$, the cosine of the neutral beam injection angle, is referred to by the two-point Simpson's rule quadrature subroutine called by function GEEF.

Cailed from: ASIMP2, referred to through EXTERNAL statement in GEEF Functions called: PCX, BFUN

Commons required: INPUT, ØUTPUT (see Appendix II)

Variable required: $\mathrm{Y}=\mathrm{v} / \mathrm{v}_{\mathrm{o}}$

Variable changed: GEEFU1 
FUNCTI $\emptyset \mathrm{N}$ GEEFU2 (Y)

Function GEEFU2 returns the value of the integrand for the second of two integrals contributing to the expression evaluated by GEEF. The expression for,the integrand

$$
\text { GEEFU } 2=2 p_{c x}(v) M^{E}(v) \frac{\left(v / v_{o}\right)^{4}}{\left(v / v_{o}\right)^{3}+\left(v_{c} / v_{o}\right)^{3}}
$$

is referred to by the two-point Simpson's rule quadrature subroutine called by function GEEF.

Called from:

ASIMP2, referred to through EXTERNAL statement in GEEF

Functions called: PCX, BMEF

Commons required: INPUT, ØUTPUT (see Appendix II)

Variable required: $\quad \mathrm{Y}=\mathrm{v} / \mathrm{v}_{\mathrm{o}}$

Variable changed: GEEFU2 
FUNCTI $\emptyset \mathrm{N}$ BKIEF (V)

Function BKIEF computes the correction to the amount of momentum transferred from the injected neutral beam to the plasma ions due to electric field affects according to the equations

$$
\text { BKIEF }=K_{i}^{E}=\frac{1}{3 \xi_{0}} \int_{v(t)}^{v_{0}} d v \frac{v_{c}{ }^{3}}{v^{3}+v_{c}{ }^{3}}\left[1+\frac{m_{i}<Z>}{m_{f}[z]}\right] p_{c x}(v)\left\{\dot{g}(v)+K_{i e}^{E}\right\}
$$

where

$$
\begin{aligned}
& g(v)=\frac{v^{2}}{v^{3} 1 v_{c}^{3}}\left[1+\left(3 \xi_{o}^{2}-1\right) b^{3}(v)\right], \\
& K_{i e}{ }^{E}(v)=\operatorname{AKI}(A) * \operatorname{BFUN}(A)=\int_{v}^{v_{0}} \frac{v^{\prime} d v^{\prime}}{v^{\prime 3+v} c_{c}^{3}} \frac{b(v)}{b\left(v^{\prime}\right)}[3-(1 \\
& \left.\left.+\frac{\tau_{s}}{\tau_{c x}\left(v^{\prime}\right)} \frac{v_{i}^{3}}{v^{\prime 3}+v_{c}} \frac{m_{i}<Z>}{m_{f}[Z]} \frac{v_{c}^{3}}{v^{\prime 3+v_{c}^{3}}}\right)\left(1+\left(3 \xi_{o}^{2}-1\right) b^{3}\left(v^{\prime}\right)\right)\right],
\end{aligned}
$$

and.

$$
b(v)=\operatorname{BFUN}(A)=\left[\frac{v_{0}^{3}+v_{c}^{3}}{v^{3}+v_{c}^{3}}\left(\frac{v^{3}}{v_{0}^{3}}\right)\right]^{\frac{m_{i}<Z>}{3 m_{f}[Z]}}
$$

and $\xi_{0}=\cos \theta_{0}$, the cosine of the neutral beam injection angle.

The remaining variables have been defined in the descriptions of FPM $\varnothing \mathrm{ME}$ and of the first few functions called by frmyivis.

Called from:

FPM $\varnothing \mathrm{ME}$

Subroutines called: ASIMP2, BKIEF1 (through an EXTERNAL rcference), BKIEF2 (through an EXTERNAL reference)

Commons required: INPUT, ØUTPUT (see Appendix II)

Variable required: $\quad \mathrm{V}=\mathrm{v}(\mathrm{t})$

Variable changed: BKIEF 
FUNCTI $\emptyset \mathrm{N}$ BKIEF1 (Y)

Function BKIEF1 returns the value of the integrand for the first of two integrals contributing to the expression evaluated by BKIEF. The expression for the integrand

$$
\begin{aligned}
& \text { BKIEF } 1=\frac{p_{c x}(v)}{3 \xi_{0}}\left[\frac{\left(\frac{v_{c}}{v_{0}}\right)^{3}}{\left(\frac{v}{v_{0}}\right)^{3}+\left(\frac{v_{c}}{v_{0}}\right)^{3}}\right]\left[1+\frac{m_{i}<z>}{m_{f}[\mathrm{z}]}\right]\left[\frac{\left(\frac{v}{v_{0}}\right)^{2}}{\left(\frac{v}{v_{0}}\right)^{3}+\left(\frac{v_{c}}{v_{0}}\right)^{3}}\right] \\
& x\left[1+\left(3 \xi_{o}^{2}-1\right) b^{3}(v)\right]
\end{aligned}
$$

where $\xi_{0}=\cos \theta_{0}$, the cosine of the neutral beam injection angle, is referred to by the two-point Simpson's rule quadrature subroutine called by function BKIEF.

Called from: ASIMP2, referred to through EXTERNAL statement in BKIEF Functions called: BFUN, PCX

Commons required: INPUT, ØUTPUT (see Appendix II)

Variable required: $Y=v / v_{0}$

Variable changed: BKIEF1 
FUNCTI $\emptyset \mathrm{N}$ BKIEF2 (Y)

Function BKIEF2 returns the value of the integrand for the second of two integrals contributing to the expression evaluated by BKIEF. The expression for the integrand

$$
\text { BKIEF } 2=\frac{1}{3 \xi_{o}}\left[1+\frac{m_{i}<Z>}{m_{f}[Z]}\right]\left[\frac{\left(v_{c} / v_{o}\right)^{3}}{\left(v / v_{o}\right)^{3}+\left(v_{c} / v_{o}\right)^{3}}\right] p_{c x}(v) k_{i e}{ }^{E},
$$

where

$$
\begin{aligned}
K_{i e}^{E}= & A K I(A) * B F U N(A)=b^{\prime}(v) \int_{v}^{v} \frac{v^{\prime} d v^{\prime}}{v^{\prime 3}+v_{c}^{3}} \frac{1}{b\left(v^{\prime}\right)} \\
& \times\left[3-\left[1+\left(3 \xi_{o}^{2}-1\right) b^{3}\left(v^{\prime}\right)\right] * B A A\right]
\end{aligned}
$$

with

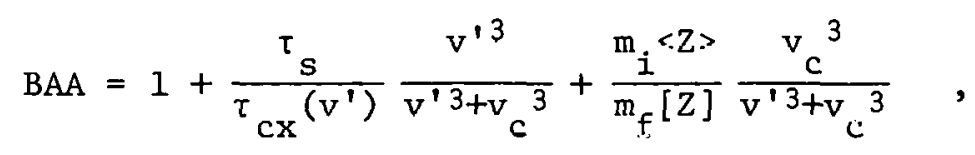

$\mathrm{A}=\mathrm{v}$,

and $\xi_{0}=\cos \theta_{0}$, the cosine of the neutral beam injection angle, is referred to by the two-point Simpson's rule quadrature subroutine called by function BKIEF.

Called from: ASIMP2, referred to through EXTERNAL statement in BKIEF Functions called: PCX, AKI, BFUN

Commons required: INPUT, ØUTPUT (see Appendix II)

Variable required: $Y=v / v_{0}$

Variable changed: BKIEF2 
FUNCTI ØN AKI (V)

Function AKI returns the value of the integral

$$
A K I=K_{i e} E^{v}(v)=\int_{v}^{v} \frac{v^{\prime} d v^{\prime}}{v^{\prime 3}+v_{c}^{3}} \frac{1}{b\left(v^{\prime}\right)}\left\{3-\left[1+\left(3 \xi_{o}^{2}-1\right) b^{3}(v)\right] * \text { BAA }\right\}
$$

where

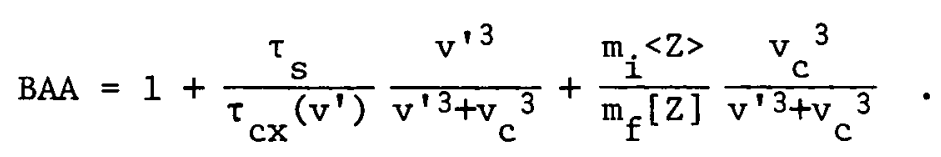

Called from:

BKIEF2, BKEEF2

Subroutines called: GAUSS, AKIF (through an EXTERNAL reference)

Commons required: GAØRD, INPUT, ØUTPUT (see Appendix II)

Variable required: $\quad V=v(t)$

Variable changed: AKI 
FUNCTI $\emptyset N$ AKIF (Y)

Function AKIF returns the value of the integrand

$$
\operatorname{AKIF}=\frac{1}{b(v)} \cdot \frac{\left(v / v_{o}\right)}{\left(v / v_{o}\right)^{3}+\left(v_{c} / v_{o}\right)^{3}}\left\{3-\left[1+\left(3 \xi_{o}^{2}-1\right) b^{3}(v)\right] * B A A\right\},
$$

where

$$
\mathrm{BAA}=1+\frac{\tau_{s}}{\tau_{\mathrm{cx}}(\mathrm{v})} \frac{\left(\mathrm{v} / \mathrm{v}_{\mathrm{o}}\right)^{3}}{\left(\mathrm{v} / \mathrm{v}_{\mathrm{o}}\right)^{3+\left(v_{c} / \mathrm{v}_{o}\right)^{3}}}+\frac{\mathrm{m}_{i}<\mathrm{Z}>}{m_{\mathrm{f}}[\mathrm{z}]} \frac{\left(\mathrm{v}_{\mathrm{c}} / \mathrm{v}_{\mathrm{o}}\right)^{3}}{\left(\mathrm{v} / \mathrm{v}_{\mathrm{c}}\right)^{3}+\left(\mathrm{v}_{\mathrm{c}} / \mathrm{v}_{\mathrm{o}}\right)^{3}},
$$

used by the Gaussian quadrature subroutine called by function AKI.

Called from:

Commons required:

Variable required: $\quad \mathrm{Y}=\mathrm{v} / \mathrm{v}_{\mathrm{O}}$

Variable changed: AKIF 
FUNCTI ØN BKEEF (V)

Function BKEEF computes the correction to the amount of momentum transferred from the injected neutral beam to the plasma electrons due to electric field effects according to the equations

$$
\operatorname{BKEEF}=\mathrm{K}_{\mathrm{e}}^{\mathrm{E}}=\frac{1}{3 \xi_{\mathrm{o}}} \int_{\mathrm{v}(\mathrm{t})}^{\mathrm{v}} \mathrm{o} \mathrm{dv} \frac{\mathrm{v}^{3}}{\mathrm{v}^{3}+\mathrm{v}_{\mathrm{c}}^{3}} \mathrm{p}_{\mathrm{cx}}(\mathrm{v})\left\{\mathrm{g}(\mathrm{v})+\mathrm{K}_{i e}{ }^{\mathrm{E}}\right\}
$$

. where

$$
\begin{aligned}
& \left.g(v)=\frac{v^{2}}{v^{3}+v_{c}^{3}}\left[1+3 \xi_{o}^{2}-1\right) b^{3}(v)\right], \\
& \mathrm{K}_{i e}{ }^{\mathrm{E}}(\mathrm{v})=\operatorname{AKI}(\mathrm{A}) * \mathrm{BFUN}(\mathrm{A})=\int_{\mathrm{v}}^{\mathrm{v}} \mathrm{o} \frac{\mathrm{v}^{\prime} \mathrm{dv^{ \prime }}}{\mathrm{v}^{\prime 3}+\mathrm{v}_{\mathrm{c}}{ }^{3}} \frac{\mathrm{b}(\mathrm{v})}{\mathrm{b}\left(\mathrm{v}^{\prime}\right)}[3-(1 \\
& \left.\left.+\frac{\tau_{s}}{\tau_{c x}\left(v^{\prime}\right)} \frac{v^{\prime 3}}{v^{\prime 3}+v_{c}^{3}}+\frac{m_{i}<Z>}{m_{f}[z]} \frac{v_{c}^{3}}{v^{\prime 3}+v_{c}^{3}}\right)\left(1+\left(3 \xi_{o}^{2}-1\right) b^{3}\left(v^{\prime}\right)\right)\right], \\
& b(v)=\operatorname{BFUN}(A)=\left[\frac{v_{o}^{3}+v_{c}^{3}}{v^{3}+v_{c}^{3}}\left(\frac{v}{v_{0}}\right)^{3}\right]^{\frac{m_{i}\langle z\rangle}{3 m_{f}[Z]}}, \\
& \mathrm{A}=\mathrm{v} \text {, }
\end{aligned}
$$

and $\xi_{0}=\cos \theta_{0}$, the cosine of. the neutral beam injection angle.

The remaining variables have been defined in the descriptions of FPMфME and of the first few functions called by FPM $\emptyset \mathrm{ME}$.

Called from:

FPM $\varnothing$ ME

Subroutines called: ASIMP2, BKEEF1 (through an EXTERNAL reference), BKEEF 2 (through an EXTERNAL reference)

Commons required: INPUT, ØUTPUT (see Appendix II)

Variable required: $\quad V=v(t)$

Variable changed: BKEEF 
FUNCTI $\emptyset \mathrm{N}$ BKEEF1 (Y)

Function BKEEF1 returns the value of the integrand for the first of two integrals contributing to the expression evaluated by BKEEF. The integrand

$$
\operatorname{BKEEF1}=\frac{\mathrm{p}_{\mathrm{CX}}(\mathrm{v})}{3 \xi_{\mathrm{o}}}\left[\frac{\left(\frac{\mathrm{v}}{\mathrm{v}_{\mathrm{o}}}\right)^{3}}{\left(\frac{\mathrm{v}}{\mathrm{v}_{\mathrm{o}}}\right)^{3}+\left(\frac{\mathrm{v}_{\mathrm{c}}}{\mathrm{v}_{\mathrm{o}}}\right)^{3}}\right]\left[\frac{\left(\frac{\mathrm{v}}{\mathrm{v}_{\mathrm{o}}}\right)^{2}}{\left(\frac{\mathrm{v}}{\mathrm{v}_{\mathrm{o}}}\right)^{3}+\left(\frac{\mathrm{v}_{\mathrm{c}}}{\mathrm{v}_{\mathrm{o}}}\right)^{3}}\right]\left[1+\left(3 \xi_{\mathrm{o}}{ }^{2}-1\right) \mathrm{b}^{3}(\mathrm{v})\right],
$$

where $\xi_{0}=\cos \theta_{0}$, the cosine of the neutral bcam injection angle, 1s referred to by the twu-point simpson's rule quadrature subroutine called by function BKEEF.

Called from: ASIMP2, referred to through EXTERNAL statement in BKEEF Functions called: BFUN, PCX

Commons required: INPUT, ØUTPUT (see Appendix II)

Variable required: $\mathrm{Y}=\mathrm{v} / \mathrm{v}_{\mathrm{O}}$

Variable changed: BKEEFI 
FUNCTI $\emptyset$ N BKEEF2 (Y)

Function BKEEF2 returns the value of the integrand for the second of two integrals contributing to the expression evaluated by BKEEF.

The integrand

$$
\text { BKEEF } 2=\frac{1}{3 \xi_{0}}\left[\frac{\left(v / v_{o}\right)^{3}}{\left(v / v_{o}\right)^{3}+\left(v_{c} / v_{o}\right)^{3}}\right] p_{c x}(v) K_{i e}{ }^{E},
$$

where

$$
\begin{aligned}
K_{i e}{ }^{E}= & \operatorname{AKI}(A) * \operatorname{BFUN}(A)=b(v) \int_{v}^{v_{0}} \frac{v^{\prime} d v^{\prime}}{v^{\prime 3}+v_{c}^{3}} \frac{1}{b\left(v^{\prime}\right)}\left[3-\left[1+\left(3 \xi_{0}{ }^{2}-1\right)\right.\right. \\
& \left.\left.\times b^{3}\left(v^{\prime}\right)\right] * B A A\right]
\end{aligned}
$$

with

$$
\mathrm{BAA}=1+\frac{\tau_{s}}{\tau_{c \mathrm{x}}\left(\mathrm{v}^{\prime}\right)} \frac{\mathrm{v}^{\prime 3}}{\mathrm{v}^{\prime 3}+\mathrm{v}_{c}{ }^{3}}+\frac{\mathrm{m}_{\mathrm{i}}\langle\mathrm{Z}\rangle}{\mathrm{m}_{\mathrm{f}}[\mathrm{Z}]} \frac{\mathrm{v}_{c}{ }^{3}}{\mathrm{v}^{\prime 3}+\mathrm{v}_{c}^{3}},
$$

$$
\mathrm{A}=\mathrm{v} \text {, }
$$

and $\xi_{0}=\cos \theta_{0}$, the cosine of the neutral beam injection angle, is referred to by the two-point Simpson's rule quadrature subroutine called by function BKEEF.

Called from: ASIMP2, referred to through EXTERNAL statement in BKEEF Functions called: PCX, BFUN, AKI

Commons required: INPUT, ØUTPUT (see Appendix II)

Variable required: $Y=v / v_{0}$

Variable changed: BKEEF 2 
FUNCTI $\phi N$ BNEF (V)

Function BNEF computes the correction to the number of stored fast ions due to electric field effects according to

$$
\operatorname{BNEF}=N^{E}=\int_{v(t)}^{v_{0}} \frac{v^{2} d v}{v^{3}+v_{c}^{3}} P_{c x}(v)\left\{\frac{\xi_{0} v_{0} v^{2}}{v^{3}+v_{c}^{3}} b(v)-M^{E}(v)\right\},
$$

where

$$
\begin{aligned}
& p_{c x}(v)=P C X=\exp \left\{-\tau s \int_{v}^{v} \frac{v^{\prime 2} d v^{\prime}}{v^{\prime 3+v}{ }^{3}} \frac{1}{\tau_{c x}\left(v^{\prime}\right)}\right\}, \\
& b(v)=\operatorname{BFUN}(v)=\left[\frac{v_{0}{ }^{3}+v_{c}{ }^{3}}{v^{3}+v_{c}{ }^{3}}\left(\frac{v}{v_{0}}\right)^{3}\right]^{\frac{m_{i}<Z>}{3 m_{f}[\mathrm{Z}]}},
\end{aligned}
$$

and

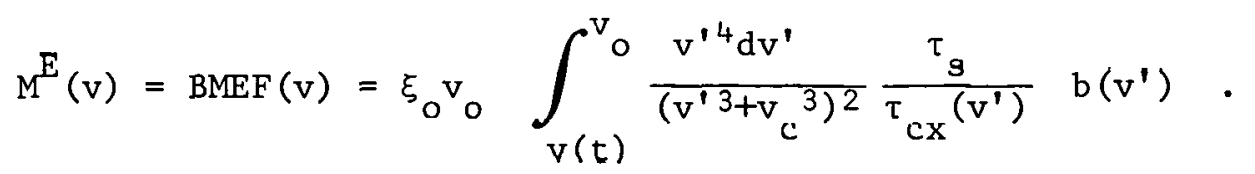

The variables have been defined in the descriptions of subroutine FPM $\emptyset \mathrm{ME}$ and of the first few functions called by FPMфME.

Called from:

FPMØME

Subroutines called: ASIMP2, BNEFU1 (thruugh an EXILINNAL reficrence), BNEFU2 (through an EXTERNAL reference)

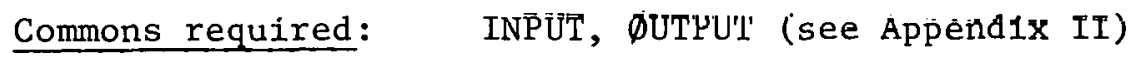

Variable required: $\quad \mathrm{V}=\mathrm{v}(\mathrm{t})$

Variable changed: $\quad$ BNEl' 
FUNCTI $\emptyset \mathrm{N}$ BNEFU1 (Y)

Function BNEFUl returns the value of the integrand for the first of two integrals contributing to the expression evaluated by BNEF. The expression for the integrand

$$
\mathrm{BNEFU} 1=2 \xi_{o} p_{c x}(v) b(v) \frac{\left(v / v_{o}\right)^{2}}{\left(v / v_{o}\right)^{3}+\left(v_{c} / v_{o}\right)^{3}}
$$

where $\xi_{0}=\cos \theta_{0}$, the cosine of the neutral beam injection angle, is referred to by the two-point Simpson's rule quadrature subroutine called by function BNEF.

Called from: ASIMP2, referred to through EXTERNAL statement in BNEF Functions called: PCX, BFUN

Commons required: INPUT, ØUTPUT (see Appendix II)

Variable required: $\quad \mathrm{Y}=\mathrm{v} / \mathrm{v}_{\mathrm{O}}$

Variable changed: BNEFU1 
FUNCTI ØN BNEFU2 (Y)

Function BNEFU2 returns the value of the integrand for the second of two integrals contributing to the expression evaluated by BNEF. The expression for the integrand

$$
\operatorname{BNEFU} 2=p_{C x}(v) M^{E}(v) \frac{\left(v / v_{o}\right)}{\left(v / v_{o}\right)^{3}+\left(v_{c} / v_{o}\right)^{3}}
$$

is referred to by the two-point Simpson's rule quadrature subroutine called by function BNEF. .

Called from: ASIMP2, referred to through EXTERNAL otatement in BNEF Functions called: PCX, BMEF

Commons required: INPUT, ØUTPUT (see Appendix II)

Variable required: $\quad \mathrm{Y}=\mathrm{v} / \mathrm{v}_{\mathrm{O}}$

Variable changed: BNEFU'2 


\section{REFERENCES}

1. R. H. Fowler, J. D. Callen, J. A. Rome, and J. Smith, FIFPC: A Fast Ion Fokker-Planck Code, ORNL/TM-5487 (July 1976).

2. J. A. Rome, J. D. Callen, and J. F. Clarke, Nucl. Fusion 14: 141 (1974); R. J. Colchin and J. Wooten, ORMAK Technical Memo 163 (May 6, 1974).

3. B. Carnahan, H. A. Luther, and J. 0. Wilkes, Applied Numerical Methods, John Wiley and Sons, Inc., New York, 1969, pp. 100-105.

4. J. D. Callen (ORNL), private communication. 


\section{THIS PAGE}

\section{WAS INTENTIONALLY \\ LEFT BLANK}


7. DESCRIPTION OF SURFACE MODULE

\subsection{PURPOSE AND STRUCTURE OF MODULE}

The surface module calculates the plasma/wall interaction. It describes the neutral gas and impurity influx resulting from the interaction of the efflux of neutral atoms and charged particles with the limiter and walls.

We describe two different versions of this module in this section. The first version is essentially a dummy routine which returns only the value of the edge neutral density to the main program. This quantity is pre-programmed as a function of time, and uses the input data variables FCDEN, FCF, and TP $\varnothing$. The second version is more complete. The processes of charge-exchange desorption and sputtering, photon stimulated desorption, electron impact desorption, and thermodesorption of the limiter are computed. However, these processes are not treated in as detailed a fashion as possible, but are included here chiefly to illustrate the function of the module.

1. Simple Model. The module consists of the single statement FCDEX $=$ FCDEN $+($ FCF-FCDEN $)(1-E X P(-T I M / T P \emptyset))$ where FCDEN, FCF, and TP $\emptyset$ are described in Sect. 1.4. FCDEX is the value at the elapsed simulation time TIM of the edge neutral density $\mathrm{N}_{\mathrm{O}}(\mathrm{a})$.

Called from: - MAIN

Commons required: NEUTRL, TIME

Variables required:

From labeled common (see Appendix II for definitions)

/NEUTRL/ - FCDEX, FCDEN, FCF

/TIME/ - TIM, TR $\emptyset$

V́ariables changed:

FCDEX - edge neutral density at time IIM 
2. Detailed Model. The plasma/wall interaction is computed in the more detailed model. The routine includes DATA statements describing the kinds of wall/1imiter provided, so each different material will require a different module. The physical processes calculated by the module are:

a) Charge-exchange sputtering. In some versions of the code, the neutral gas module is used to compute the spectrum of emergent charge-exchange (hot) neutrals, $f_{h}(v, \theta, a)$. This spectrum is then integrated with experimental values of the energy and angle dependent sputtering yield to calculate the impurity flux which results:

$\Gamma_{z}\left(\frac{\text { number }}{c m^{2} \sec }\right)=2 \pi \int_{0}^{\infty} \mathrm{dv} \cdot \mathrm{v}^{2} \int_{0}^{\pi} \mathrm{d} \theta \sin 0 . v \cos \theta \mathrm{f}_{\mathrm{h}}(\mathrm{v}, \theta, \mathrm{a}) \mathrm{Y}(\mathrm{v}, \theta)$.

The geometry $\mathrm{v}, \theta$ is described in the introduction to Sect. 4. For this version, however, we will use results from more detailed calculations to note that the number of impurities produced per eV of charge-exchange energy is nearly constant, so we will provide this quantity in DATA statements. Thus, the impurity flux is

$$
\Gamma_{z}{ }^{\mathrm{cX}}=6.25(10)^{21} \overline{\mathrm{Y}}|\mathrm{PSCX}| / \mathrm{AREA}
$$

where PSCX is the total charge-exchange loss (in $\mathrm{kW}$ ) from PIIL (calculated in subroutine $\emptyset \mathrm{RMPWR}$ ) and PBCX (calculated in subroutine BEAM). $\bar{Y} \quad$ - number of impurities per eV, given in a DATA statement AREA - torus surface area

b) Charge-exchange desorption. For this quantity we add PHICX to the data, representing the number of impurities desorbed/chargeexchange neutral. The routine first finds an approximation to the mean energy of charge-exchange loss by finding the radius with maximum charge-exchange emission. Then the flux of charge-exchange particles is:

$\Phi_{\mathrm{CX}}=6.25(10)^{21}[\mid \mathrm{PHIL} / / \mathrm{TCX}+\mathrm{PBCX} / \mathrm{EBCX}]$

where 
PIIL is calculated in subroutine $\emptyset \mathrm{RMPWR}$,

TCX is the mean neutral energy, and

$\mathrm{PBCX}, \mathrm{EBCX}$ are the charge-exchange loss from the beam (kW), and mean energy of lost particle (eV), respectively.

The resulting impurity flux is:

$$
\Gamma_{\mathrm{CX}}^{\mathrm{CXD}}=6.25(10)^{21} \overline{\mathrm{Y}}_{\mathrm{D}}(\mid \mathrm{PIIL} / \mathrm{TCX}+\mathrm{PBCX} / \mathrm{EBCX}) / \mathrm{AREA} \text {. }
$$

c) Photon-stimulated desorption. For this process we use the mechanism whereby photoelectrons, produced by photons in the 10-100 eV energy range, bombard the walls. We consider two sources of photon emission: photons from $0^{\mathrm{VI}}$ resonance line radiation (1032 $\AA$ ) and from hydrogen Ly alpha emission. Thus we have the sources PLR, computed in subroutine $\emptyset \mathrm{RMPWR}$ from the oxygen emission, and PHY, computed in subroutine SURFAC. The photon fluxes are

$$
{ }_{0} \mathrm{VI}=6.25(10)^{21} \mathrm{PLR} / \mathrm{E} \emptyset 6
$$

where

$$
\mathrm{E} \emptyset 6=12 \mathrm{eV} \cdot
$$

The photon flux for Lya must be computed. The rate coefficient is computed in FUNCTI $\emptyset \mathrm{N}$ SLYAD. The photon flux emission is integrated over the plasma volume to obtain

$$
\Phi^{\phi}=2 \pi \int_{0}^{3} \mathrm{dr} \cdot \mathrm{r} \mathrm{N}_{\mathrm{e}} \mathrm{N}_{\mathrm{o}} \mathrm{S}_{\mathrm{x}}\left[\mathrm{T}_{\mathrm{e}}(\mathrm{x})\right] .
$$

The impurity stimulation rate is characterized by the basic quantum efficiency QEFF defined in a DATA statement. Thus the impurity flux is

$$
\Gamma_{z}^{\phi}=\mathrm{QEFF} *(\mathrm{PHI} \emptyset+\mathrm{PHILA}), \quad\left(\text { \#/ } / \mathrm{cm}^{2} / \mathrm{sec}\right) \text {. }
$$

d) Charged-particle desorption. The plasma ions and electrons which leave also produce desorption characterized by the efficiencies $\mathrm{QPR}\left(\begin{array}{l}W \\ L\end{array}\right)$ and QFT.F. $\left(\begin{array}{l}W \\ L\end{array}\right)$ [number of impurities produced per proton and per electron, respectively (for wall and limiter)]. The emergent fluxes of protons and electrons are assumed to be equal, and are computed from the quantities PCVE, PCVI in subroutine ØRMPWR. The relative fraction of this flux going to the wall. (limiter) is described by the constant in DATA PCTWAL (1-PCTWAL). Thus, the particle desorption contribution to the impurity flux is: 


$$
\begin{aligned}
& \Gamma_{z}^{P}=(P C V E *((Q E L E W * P C T W A L+Q E L E L \\
& \text { *(1-PCTWAL))/TE (N) + PCVI } \\
& \text { *(QPRW*PCTWAL + QPRL } \\
& \text { *(1-PCTWAL ) )/TI(N)) } 6.25(10)^{21} \text {. } \\
& \text { e) Total. The total impurity production is thus } \\
& \Gamma_{z}=\Gamma_{z}{ }^{\mathrm{CX}}=\Gamma_{z}^{\mathrm{CXD}}+\Gamma_{z}^{\phi}+\Gamma_{z}^{P} .
\end{aligned}
$$

This flux is treated in two ways, depending whether or not a detailed impurity model is being used $[\operatorname{IMP}(1)=1$ or 2$]$. With $\operatorname{IMP}(1)=1$, the ZEFF value is incremented uniformly by addition of the new impurity rlux $T_{z}$. The rule is

$$
\dot{\mathrm{z}}_{\text {eff }}=\dot{\mathrm{N}}_{\mathrm{z}} \mathrm{Z}^{2} / \mathrm{N}_{\mathrm{e}}
$$

where $\mathrm{N}_{\mathrm{e}, \mathrm{z}}$ are the total numbers of electrons and impurities, $\mathrm{Z}$ (ZIMP) is the average $Z$ of the impurity species and $\mathrm{N}_{z}=\Gamma_{z} \times$ AREA. With the detailed model the impurities are added to the species being monitored. Thus one can discuss heavy metals (from the wall or limiter), light impurities (from desorption), or hydrogen. For this discussion we will treat only the case of light impurities produced by the sum of all the processes discusscd above. In fact, we will assume the impurity to be $C A R B \emptyset N$, and the impurity is to be added to the CARB $\emptyset \mathrm{N}$ array in the last spatial zone. Thus, the increment of carbon atoms produced during time $\Delta t$ is

$$
\text { TDELC }=\Delta \mathrm{t}^{*} \Gamma_{z}{ }^{* \mathrm{AREA}}
$$

and the density in the outer spatial zone is

$$
\operatorname{DELC}=\operatorname{TDELC} / 2 \pi \mathrm{R}_{\mathrm{o}} 2 \pi \mathrm{a}(\mathrm{HR})
$$

\subsection{BASIC INPUT/OUTPUT}

The routine requires some of the major energy and particle fluxes from the plasma which are calculated in subroutine $\emptyset$ RMPWR. It calculates either an increment in ZEFF [if $\operatorname{IMP}(1)=1$ ], or an increment in the $\operatorname{CARB} \emptyset \mathrm{N}(\mathrm{N})$ density $[$ if $\operatorname{IMP}(1)=2]$. 


\subsection{SUMMARY OF FUNCTION OF EACH SUBROUTINE}

SUBRøUTINE SURFAC

Subroutine SURFAC is currently the only subroutine in this module.

Called from:

Commons required:

\section{MAIN}

IMPURT, INDEX, NEUTRL, TIME, SURF, PøWER, ØUTPT, RATI $\emptyset S, G E \emptyset M, I \emptyset N S$

\section{Variables required:}

From labeled common blocks (described in Appendix II):

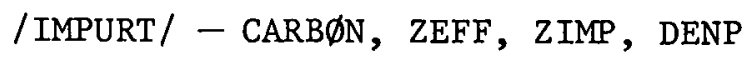

$$
\begin{aligned}
& \text { /INDEX/ }-\mathrm{N} \\
& \text { /TIME/ - TIME, TP } \\
& \text { /NEUTRL/ - FCF, FCDEX, FCDEN } \\
& \text { /SURF/ - CARN } \\
& \text { /POWER/ - PED, PID, PIIL } \\
& \text { /ØUTPT/ - PLR } \\
& \text { /RATI } \varnothing \text { / - PCVE, PCVI } \\
& \text { /CEøM/ - AREA } \\
& \text { /IØNS/ - DEN }
\end{aligned}
$$

\section{In a DATA statement:}

$$
\begin{aligned}
& \text { YBAR - number of impurities per eV } \\
& \text { YBARC - number of impurities of carbon per } \mathrm{eV} \\
& \text { QEFF - basic quantum efficiency } \\
& \text { QELE }\left(\begin{array}{l}
W \\
\text { L }
\end{array}\right) \text { - electron desorption efficiencies for wall (W) and limiter (L) } \\
& \mathrm{QPR}\left(\begin{array}{l}
W \\
\mathrm{~L}
\end{array}\right) \text { - ion desorption efficiencies for wall (W) and limiter (L) } \\
& \text { YBARD - number of impurities from charge-exchange desorption per eV }
\end{aligned}
$$

\section{Variables changed:}

$$
\begin{aligned}
& \text { ZEFF - effective charge } z_{\text {eff }} \text { of plasma ions } \\
& \text { CARN - edge density of carbon atoms, } \mathrm{cm}^{-3}
\end{aligned}
$$




\section{THIS PAGE}

\section{WAS INTENTIONALLY LEFT BLANK}




\section{DESCRIPTION OF DIAGNOSTIC MODULE}

\subsection{PURPOSE AND STRUCTURE OF MODULE}

Development of this module has just begun. It will contain all the diagnostic procedures, checks on numerical accuracy, error messages, and diagnostic printout of variables and arrays to help locate where problems are occurring during a simulation run. Three levels of diagnostics will be considered in order of increasing severity of problems encountered.

Level one diagnostics. This level includes checks on numerical accuracy, some consistency checks and associated error messages. Errors occurring at this level will not stop a simulation run unless the user specifies that this be done by his choice of input data for setting various switch variables. The following numerical accuracy checks are automatically made:

a) The computed current

$$
I=2 \pi \int d r r j(r)
$$

is compared to the program current.

b) The quantity $n\left(j-j_{B E A M}\right)$ is compared to the electric field $E$.

c) The quantity

$$
-(N V) /\left(D_{\frac{\partial N}{2 r}}\right)
$$

should be unity.

Level two diagnostics. This level will contain diagnostic procedures and associated error messages that can be used to attempt to recover if the simulation is going badly. Problems encountered at this level will be more serious. An option will exist for stopping the program by the choice of input data for various switch variables.

Level three diagnostics. This will be the level of diagnostics for the most serious problems. This level will be used when extensive diagnostic information is required in order to locate the source of a problem. It must be requested deliberately by the user since it will usually generate a large amount of output. 


\subsection{SUMMARY OF FUNCTION OF EACH SUBROUTINE}

The only subroutine at present is DIAGN $\varnothing$. It provides a few level one and one level three diagnostic aids.

SUBR ØUTINE DIAGN

Subroutine DIAGN $\emptyset$ contains the three level one diagnostics mentioned above. A level three diagnostic was added when some problems occurred in subroutine IBTM in the plasma module, and checks for vanishing matrix elements in the coefficient matrix A of the set of finite-differenced coupled partial ditterentià équations describing the plasima evululiuk. If a vanishing matrix element is found, it is given the value 1.0 and the location of that element is printed out using alcernace ENTRY DPRT. This allows the program to continue even though the simulation is in error, so that further degeneration of the computation can occur and perhaps yield more helpful diagnostic information. Alternate ENTRY DAPRT examines the coefficient array for elements $>10^{40}$ and prints any that are found.

Called from: MAIN, IBTM [alternate ENTRY DAPRT]

Commons required: BEAMC, CDCLBX, FIELDS, GE $\emptyset \mathrm{M}$, INDEX, I $\emptyset \mathrm{NS}$, LBMIBT, SUMS, 'I'E'MP, THRMDF

Variables required:

For ENTRY DPRT (KDUM, IDUM, L $\emptyset \mathrm{P}$, VALUE)

KDUM - a variable used to isolate which group of statements was being executed when trouble occurred. The value of this variable is printed during program execution and is also a useful aid when examining the contents of a core dump when execution fails.

IDUM - the product of the $\mathrm{D} \emptyset$ loop indices $\mathrm{I} 1^{*} \mathrm{I} 2$ in subroutine IBTM. The valuc of IDUM is printed during program execution if the value of certain elements of the coefficient matrix $A$ are $-10^{-40}$.

$L \emptyset \emptyset \mathrm{P}$ - index value of the A-array element causing diffiru1ty.

VALUE - value of the quantity 1 ./A(L $\emptyset \mathrm{P})$.

From labeled common blocks (See Appendix II for definitions)

$$
\begin{aligned}
& \text { /BEAMC/ - DJT(101) } \\
& \text { /CDCLBX/ - ETA (101) } \\
& \text { /FIELDS/ - ZJ(101) }
\end{aligned}
$$




$$
\begin{aligned}
& \text { /GE } \emptyset \mathrm{M} / \quad-\mathrm{AM} \\
& \text { /INDEX/ - N } \\
& \text { /IøNS/ - DEN (101) } \\
& \text { /LBMIBT/ - A(8008) } \\
& \text { /SUMS/ - SUMC } \\
& \text { /TEMP/ - VEL(101) } \\
& \text { /THRMDF/ - TDE (101) }
\end{aligned}
$$

Variables changed:

RCUR - ratio of computed current to program current (should be unity).

SIGV - ratio of plasma ion flux computed from drift velocity to flux computed with thermal diffusion coefficient (should be unity). 
APPENDIX I

I. 1 


\section{2}

Appendix I contains a listing of the input data set used by the transport code. This listing contains two parameters, SCAIJ and SCAEPI, which are not described in the User's Manual. They appear with the parameters listed under the heading NEUTRAL GAS DATA and are included in the NAMELIST 1ist I NEGADA | in subroutine GETSET. They are not used in the version of the transport code described in this manual. Except for these two parameters, the rest of the listing should agree with the description in the. User's Manual text. 
THIS IS THE OPPICIAL INPOT DATA SET FOR THE VERSION OP THE OAR BIDGE TOKAHAK TEANSPOBT CODE AS DESCRIBRD IN THE OSRR'S MANOAL.
I N F U T
C. $T$ A
A $N$
$S$ I I C H
$S$ B T T I N G S

THE POLLONI IG INPOT DATA SET IS COMPLETELY IHTERNALIY DOCOHENTED SO THAT THE CASUAL OSEB SHOOLD BE ABLE TO EUA THE PL AS HA S IMULATION CODE HTTHOUT HAVIHG TO CONSULT A SBRIES OP HENOS. CHANGES TO THE INPUT DATA AQE HADE BY BDITING THIS PIIE, I. B. THE NAUELISI LISTS.

THE VARIABIES APPEOPRI ATE POB EACH YABEIIST LIST GROUPIHG ABE DESCBIBED IMEEDIATELY PRECEEDING THE NAMELIST DATA SET. DERAULT VALUES POR THE IHPOT DATA ARE GIVEN IA PAREMTHESES POLLOTI HG THE

DEPINITIONS OP THE VARIABLES. THE DEPAULT UALUES GIVBA ABE THOSE

APPROPBIATE POE THE OBHAK DEVICE.

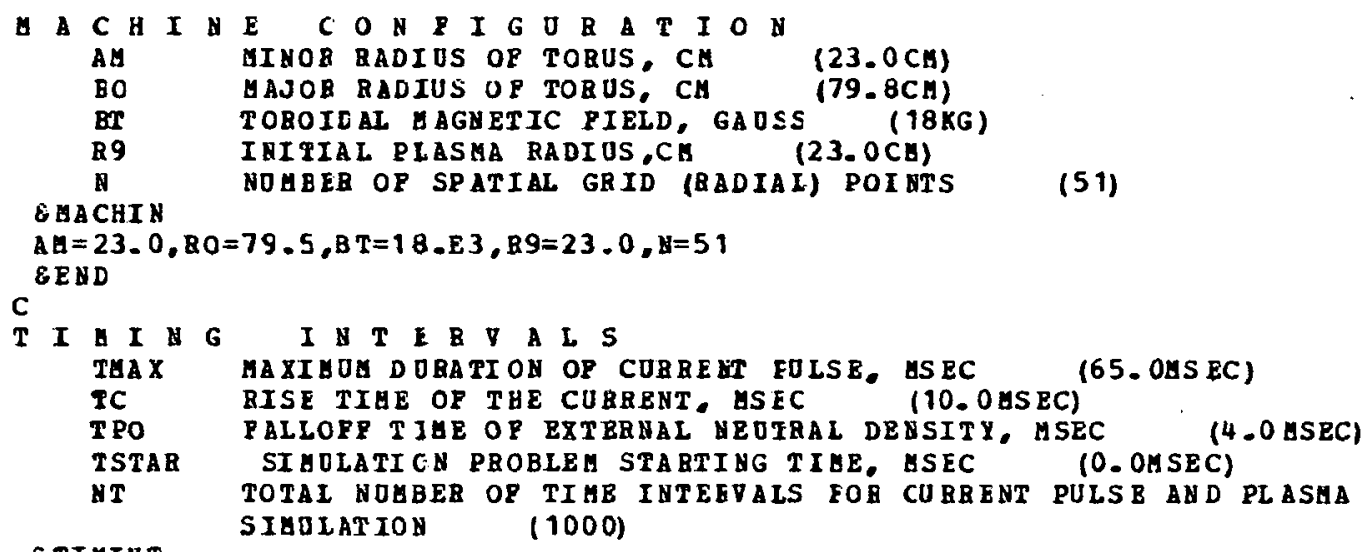

\section{$5 \operatorname{TIAINT}$}

$T \Delta A X=65.0, T C=10.0, T P O=4.0, T S T A B=0.0, H T=1000$ SEND

C

P I A S I A

TEO

TIO

TRB

TIB

YOLT

DENO

DE\B

ZPC

ZFI

AHO

*** NOT $B$

$* * *$

$* * *$

D A T A

EOU NIABY TEEPEBATURE FOR ELBCTRONS (IONS), EV BOO $\triangle D A B Y$ AND INITIAL TEKPBRATORE OF IONS, EV IAITIAL CBETBAL VALUE OF RLECTBCN TEMPERATORE, BY (20.0BV) INIT IAL CENTRAL VALUE OP ION TEMPEBATURE, EV (20.EV) TOBOIDAL VOLTAGE, $\nabla$ (3.OV)

INITIAL VALUE ZOR DENSITY AT FLASHA CENTER, CA**-3 EOU XIABY ELECTRON DBUSITY,CH**-3 (5.E 12CA**-3)

IHITIAL VALOE OP THE TOTAL C UBRENT, ABPS (15.0KA)

ADDITIOUAL A HOUNT OP TOTAL. CUREET AFTEB TIAETC, AMPS

HASS OP PLASHA IO AS (SEB TABIE FCLLONING). AHU (1.0A4O)

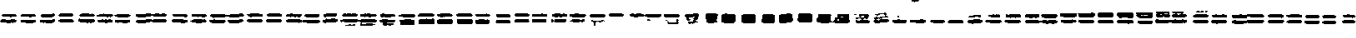
PILIING GAS AMU

100X HYDROGE

75\% BYDBOGEU, 25X DEUTEBIOH

1.0

5O\% HYDROGBY, 5OX DEOTERIUI

1. 25 ETC.

1.50

$X I(1)$

$X I(2)$

$X I(3)$

$X I(4)$

$\operatorname{INIT~(1)~}$
BXPONENT ROR INTTAL PROFILE CP COI

$$
\begin{aligned}
& \text { CORAENT DENSITY (2.0) } \\
& \text { ELBC TRO IE TE. (2.0) } \\
& \text { ION TEUP. }(2.0) \\
& \text { ELECT EOA DERSITY }(2.0)
\end{aligned}
$$

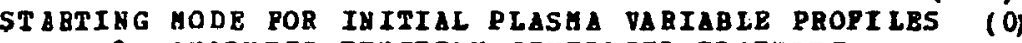

$$
=0 \text {, AHALYTIC POUCTION GEREAATED PBORILES }
$$

$=1$, PBORILES READ FECA DAT A CABLS

IUIT (2) УOT CUBAENTLI USED

IHIT (3) SRITCG POB EIAGIMIAG ABD STABILITY CHARACTBRISTICS OP THE PLASAA

$=0$. DET BRA II BS LOCATIOY OP SIMG OLAR SORPACB POR $\mathrm{E}=3,5$

$=1$. CALCOLATBS BADI AL ETGEBPO BCTICAS AND TBARING RaT a ICCORDIA 0 TO TORTH, DOTHEHYOED, AND SELBERG, FOR H=3.5. CAL BE USBD TO BV ALOATE TELISPOBT COEREICIBNTS IA SUBDOOTIHE CDC. 
D11(1) ELECTRON THERGAL TRANSPORT CORFEICIENT, ROR SCALIAG DIPPUSION

COEEFICIENT = D11(1) * PSEUDO-CLASSICAL $(0.350)$

D11 (2) ION THERMAL TRANSPORT COERFICIENT, FOR SCALING DIFPOSION

$$
\text { COEPFICIEAT = D11(2)* } \mathrm{NEO-CLASICAL}(0.300)
$$

D11 (3) PARTICLE DIFFUSION COEPPICIENT = 1.00 * PSEUUO-CLASSICAL

D11 (4) RESISTIVITY = D11(4)* NEO-CLASSICAL (1.00)

DII (5) THBESHOLD (HUE*) OF TRAPPED EIECT FON SCALIMG $(0.0)$ EFLADAT

D11 (6) THRESBOLD (NOI*) OP TBAPPED ICA SCALING (0.0)

$T E O=10.0, T I O=10.0, T B B=20.0, T I B=20.0$, VOIT $=3,0, D E X O=6 . E 12, D E N B=5 . E 12$.

$\mathrm{ZPC}=1.5 \mathrm{E} 4, \mathrm{ZFI}=8.5 \mathrm{E} 4, \mathrm{AH} \mathrm{D}=1,0, \mathrm{XI}=2.0,2.0,2.0,2.0,0.0,0.0,0.0,0.0,0.0,0.0$,

I NI T $=0,0,1,0,0,0,0,0,0,0$,

D 11 $=0.350,0.300,1.0,1.0,0.0,0.0$

EEND

A E U T B A I G A S D A T A

PCDEX (TIA) $=($ PCDEN-PCP) EXP (-TIVTPO) + PCP

FCDEX FBABCK-COEDON BOUNDARY DEUSITY OF IBUTRALS AT TIAB=TIE

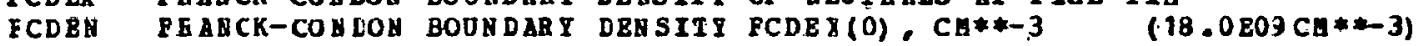

FCY FINAT. AOUNDARY DEMSITY OF ERUTRALS RCDEX (INP), CH**-3 (9.0EO9CH**-3)

NOX NUBER OF POIBT3 I $[0,1]$ AT HICU TO APRBOXIMAT BOT NEOTRAL GAS DEHSITY (21)

SOZ NUABEA OF ORIFORHLY DISTAIBOTED POIHTS IV [-1,1] OSED TO APPBOXI BATE HOT NEOTRAL GAS LE ISITY (10 1)

ISIMP NUABER OP SIUPSON-XODES OSBD TO APPROXT AATE AN I BTEGRAL NEAR A LOGABITHAIC SINGOLARITY IA G(Q) (9)

NOAIF SEITCH POR THE NODAL POIATS IN [0,1], (0)

$=0$, POINTS ARE DERIHBD IY NBOTIO SOBAOOTIBE $20 \mathrm{BE}$ $(I-1) /($ NOX -1$)$

$>$ 0, POINTS SHOULD BE ELACED AT THB BEGI ABI G OP TBE ABRAY 1 IN COMBON / ITRLP/

QP. QC DETERGI ME AN I NTEGRATTOA INTERVAL ON EACH SIDE OP THE LOGARIT HAIC S IN GULABITZ OF G(Q) AT EACH MODAL POIAT IN $[0,1]$. RBQUIRE $O<O R=Q P<Q C$. WENEVER QF> QC, NEUTIO SETS $Q P=0.1 * O C$. $\quad(0.001,0.11)$

BPSI CONVERGE CB CRITBEION FOR HOT NEOTRAL GAS DENSTTX SOLUTIOA TO THE DI SCBETE IUTEGBAL BGO ATIOS (0.001)

I UT EPS $=1,2,3$ SEL BCTS RESPECTIVELY LIHEAR, QUADRATIC, OR CUBIC I HTEROLATION POB APPBOXIAATIHG HI, TI, AMD BATY (2)

BSH 0 = BUABBA OP DIPISTOB POI UTS IB TEB 2 PABTITIOA IATER VALS THAT COATAI THE POT $\triangle$ OP THE LOGABITAUIC SIHGOL ARITY (3)

IDEDUG $\rightarrow 0$ POB MO IR POREATION

=1. PRI ATS $A=P 1, B=P 2, C=A P C$, LIPSHIT COHSTABT, ESTIEATED HOEBER OP ITBRATIONS

=2. PRINTS SAM AS =1 PLOS AREAYS HI, TI (OB AI), ADD BATI

BO ENE EGY OF MB OTRALS OPF THE HALL RY. 110.1

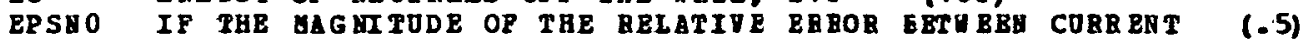

$A * A I / A I$ ALD OLE OSED AT PRETIOUS TIAE TO GET LAST BASIC

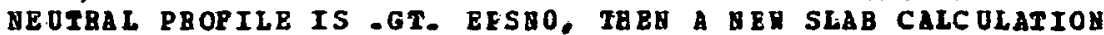

QILL BE $\triangle \triangle D B$

IENO AFPLICABL B ONLY IF NGQ SLAB CaLCUATION IS TO BB MADE

$=0$ POR HO PBI KT

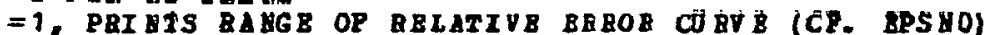

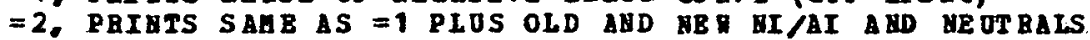

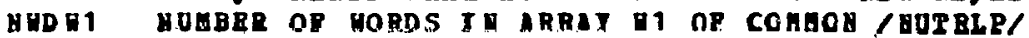

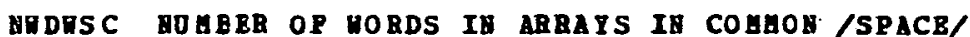

HOTE (1) SEITCH FOB BODEL OP HETRAL GAS CALCOLTIOH

$=1$ POB ABALYIC $\triangle O D B L$

$=2$ POA SLAB MODEL (JOBB BOGAN), HO REPEBCTIOE HADE $1, G E .(5 \mathrm{BH}$. $\mathrm{BOX}+3 \mathrm{HOZ})$

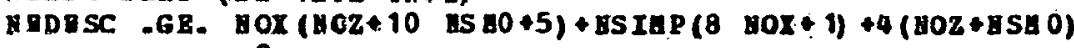
$-2$

$=3$ POB SLAB HODEL IITB H. EOBIHSOR'S HALI RBRBCTTOB

(DIER BR SIGHAR, NOT AVAILABLE YBT)

BIDI 1 -GB. $(5 B+580 \mathrm{X}+3 \mathrm{BOZ})$

BDDESC - GB. (15 $\mathrm{BOX}+8 \mathrm{BCZ}+\mathrm{BOX} * \mathrm{BCB}$ )

$=4$ POR. XCDRA VERS TOA OP ASISE PBOGBAB (BOT IBCIDDED YBT) 
SCAIJ SCALING FACTOR FOR THE I (AND J) PONCTION APPEABING IN THE REFLECTION PART OF THE KEENEI. APRIIES IP NUTB $(1)=3$.

SCAEP 1 SCALING FACTOR FOR THE PUNCTION EPSILON SOB 1 THAT APPEARS IN TEE BEFLECT ION PART OF TBE KERNEL, APRLICABLE ONLY IF NUTE (1) $=3$.

NUTE(2) EXEONENT IN POWER LAH ZOR NBUTRAL GAS ANALYTIC DODEL

H.B.** IF NO SLAB CALCULATION THEN LAEBLIET COMMOHS /INTRPS/. /PBNT/ ARE MOT USED ANDTHE ABRAY IN/NUTBLP/ SHOULD HAVE EXACTLY 1 ELEH BNT.

SXIT, IPBIT, EGGT1, AND BNT SHCUID EE SPECIPIED DHEN EVER JOHA HOGAN'S SLAB MODEL IS REQUESTED. IPEIT, NC, EGGT 1, AND BNT APPLY ONIY IP GAMMA (=LIPSCHITZ CONSTANT) -GE. I OR IF THE ESTIMATED NOMBER OP FUNCTICNAL ITEBATIOAS EXCEEDS UXIT. NEUTC SETS NC TO 1 (O) IF FAILORE (SOCCESS). IT=NOIT IS THE NUABER OP PUNCTIONAL ITERATIONS PERRORAED IN NEUTC.

PRINT ROU OF I APORHATI OU ON ITEBATIONS $=1+(J-1) * I P R I T, J=1, \ldots$ DNTIL CONVERGENCE OR. PAILURE IS DECLARED. N 1 DENOTES THE HOT NBUTRAL DENSITY PUNCTION - CONVEBGENCE IS DECLARED MAE EVER SUA FOB I=1..... NOX OF ABS (NICUE(I) - NILST (I)) - LE. (SOH OP NICDR(I)) *EGGT1. IF MAX(N1COB(I)) -GB. BUT, THBN PAILORE IS DECLAEED.

SHEGADA

$P C D E N=18 . E 9, F C F=9 . E 9, N O X=21, N O Z=101, N S I M P=9$, NUNIF=0,QP=0.001,

$Q C=0,11, E P S \triangle I=0.001$, UT $B=1,4,0,0,0,0,0,0,0,0, I$ TBP $S=2$, US $\triangle 0=3, I D E B D G=1$.

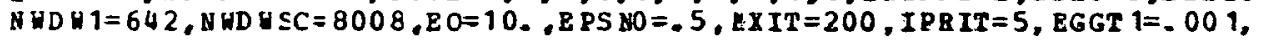
$B N T=10$, , S $C A I J=1$., SCAEP $1=1$.,

SEND

C

I D U R I T Y I O N D A T A $\begin{array}{ll}\text { ECIMP } & \text { RERCENTAGE OF ELECTRON DENSITY DUE TO A HIGH-Z IAPUAITY. } \\ \text { THIS PABAHETER HAS THO DIPPEREN T AEANINGS ACCOBDING TO THE }\end{array}$ IEP ORITI DIPFOSION MODEL CPTICA DETEBAINED BT TAE VALUE OP IUP (2). FOR IAP (2)=1 OR 3, VALUE IS DUE TO SOLE E VEBAGE EFPECTIVE HIGH-Z IAPORITI; FOB IUP(2) $=2$ OR 4, IT IS.DUE TO IRCN IAPUBITIES.

ZEFP EFFECTIVE CHABGE OF IONS (4.0)

ZIMP AAIQUA CHARGE OP THE BIGH-Z IHEOEITY ION (25.0)

XCAR PERCENTAGE OF ELECTROA DENSITY DUE TO CAGBON IM PUAITIES (APEEOX. 2X)

XOX PERCENTAGE OP ELECTROA DENSITY DUE TO OXYGEN IAPORITIES (APPEOX. 2x)

IUP (1) SRITCH POR INITIAL IUPUBITY SIRIPPING CALCOLATICA $=1$ COEGNA EQUILIBRI OA TABIE POR CAREON, OXYGEN

$=2$ DYNAMIC RATE CALCULATICN POB CARBON, OXXGEN (NOT AN OPTION IN CORBENT VERSION OF THE PROGRAM)

ILP (2) SHITCH FOR DIPPUSION OP IMFURITIES (1)

$=1$ A COBONA EQUILIBRIUG TABIE TOGET BER NITE RADIAL DISTRIBUTI ON PORHUIAE ABE USED POR CARBON AH D OXYGEN HIGE-Z IUPURITIES ABE TREATED OSING A CRUDE ESTI HATE

$=2$ COFONA RCOILBRIUY TABLES OSED POR CARBON, OXYGEN, AND A HIGH-Z IRPUBITY (FE) ATOH CHARGE STATES. BADIAL DISTRIBUTIONS ABE ASSIGNED ESING POBHOLAE.

$=3$ A CORONA EQUILIBRION TABIE TCGETHBR WITH BADIAL DISTBIBOTIONS GENERATED BY PRIRSCE-SCHLOTER DIFEUSIOA IS USED FOB CABBCN AND OXYGEN. HIGHZ IXPURITIES ABR TREATED USING A CRUDE ESTIHATE.

$=4$ COBONA ECUILIBRIUA TAELES ARE USBD POR CABBON, OXYGEN, AND A HIGH-Z IHPUBITY (PE) ATUN CHAKGE STATES. RADIAL DISTRIBUTIONS ARE GE \&EETED EY A PPIBSCH-SCHLUTER DIREUSIOA MODEL.

IMP (3) SEITCR POB IUPURITY $\angle O D E L$ CALCOLATIOH

$=1$ DSE PIXED VALUES

$=2$ USE MODEL CALCOLATION DETERAIUED BY CHOICE OF SRITCH PALUE POR IUP(2).

IUP (4) SRITCH FOB DETAILED GODEL FOB IBON

$=0$ INCLODE I BOA COBONAL BGOILIEAIA

$=1$ OHI T DETAILED DESCBIFTICA

EI I PIDA

$P C I A P=0,10, Z E P F=4.0,2 I A P=25,-0, X C A R=2,0, X C X=2,0, I A P=1,1,1$, EEND 
C

E U T R I B E A A I N J E C T I O N D A T A

CURBM 3 X 2 ARBAY OF NEOTRAL BEAL CUEETTS (SEETABLE BELOH), AMPS

ENGI (1) HIGEEST NETTRAL BEAU ENEBGY, EV

(3. OB4 EV)

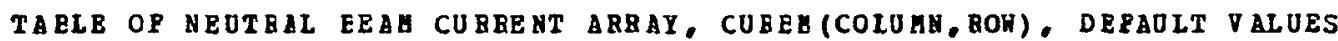

$\begin{array}{lccc} & \text { ENGI }(1)=E O & \text { ENGI }(2)=E O / 2 & \text { ENGY }(3)=80 / 3 \\ \text { CO-INJECTION } & 1.2 & 1.2 & 1.2 \\ \text { COONTER-IHJECTION } & 1.2 & 1.2 & 1.2\end{array}$

DTC B TIME INCREGENT FOR CALCULATICA OF B(B), ASEC (10.0HSBC)

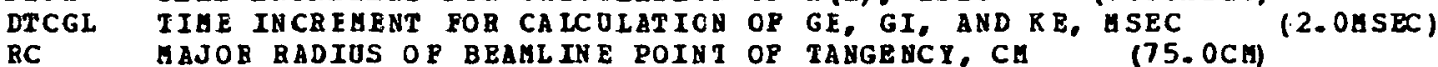

RB NEUTRAL BEAL BADIUS, CH (15.0CA)

BHLP HALF - VIDTH (ASSOGIXG GAUSSIAH PROPILB) , CH (7.5CM)

THJCT STAETIRG TIME POR INJBCTION, MSBC (20MSBC)

DTNJCT NEOTRAL BEAH INJECTION COBEENT EISE TIHE, BSEC (6.0ASEC)

N 1 MUBBER OF POINTS IV CURAEHT EISE INTERY IL (500)

\$3 WUEER OP POINTS IN IHJECTED NEOTRAL BEAV RISE TIME INTERUAL

PQE RBACTION OF IAJBCTED NEOTEAL EEAM POBER TRANSFERBED (0.95)

TO ELECTRCSS (USED FOR QUICK ASD DIETY INJECTION CALCULATIONS)

Tก.T (1)

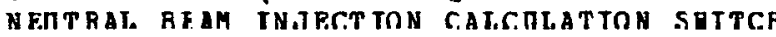

(1)

$=0$, HO I UJ.BCTION

$=$ i. ACAR NTS CALCOLATICH

= 2. DEEOG CALCULATION

IEJ (2) SEI TCB FOR NUABER A KD TYPB OF NEUTRAL BEAU IHJ BCTORS USBD

$=1$, CO-INJ RCTION OULY

= 2. COOHTER-IHJ ECTION ONLY

= 3. THO INJBCTOHS ONLY, CHE CO AND OHE COURTER-

= 4, FOUR INJECTORS, THO CO- AND IRO COUNTER-

POB THB FOLLONING SHITCBES, $1=$ OA AND $0=$ OPP

IXJ (3) SHI TCA FOR CORBERT PERTOREATICA P.BOA FAST IOAS

IUJ (4) ELECTRIC PIELD ERPBCTS OY FAST IOAS

(1)

I 1 S (5)

LOSS CONE CORBRCTION TO BAST IOHS THERAALI 2ATIOA 10

IBS (6)

SDITCB POR TYPE OP IHJECTED GAS

(0)

$$
=0 \text { HYDROG BN }
$$

$=1$ DEUTERIOH

\section{GABAIDA}

EAG Y (1) $=30000,0, D T C B=10,0, D T C G L=2,0, E C=75,0, B B=15,0$

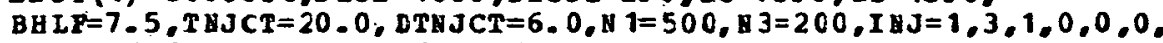

CURBG $(1,1)=1,2, \operatorname{CURB}(1,2)=1,2, \operatorname{CURBA}(2,1)=1.2$,

CURBA $(2,2)=1,2, \operatorname{CORB}(3,1)=1.2, \operatorname{COBB} B(3,2)=1.2$,

FQE=0.95
\&EHD
C
O

8 IODATA

$B E=5$

GER D
DA T2

A I D

C 0 I $\mathrm{T}$ O I

$S$ I T C B S

MOEBER OP PBIAT OOT SETS DORIMG SIMULATIOA OF PLASHA DT SCAABGE (5) 


\section{APPENDIX II}

A. Names and Primary Functions of Labeled Common Blocks

B. Identification of Variables and Arrays in Labeled Common Blocks

II. 1 
II. 2

A. Names and Primary. Functions of Labeled Common Blocks

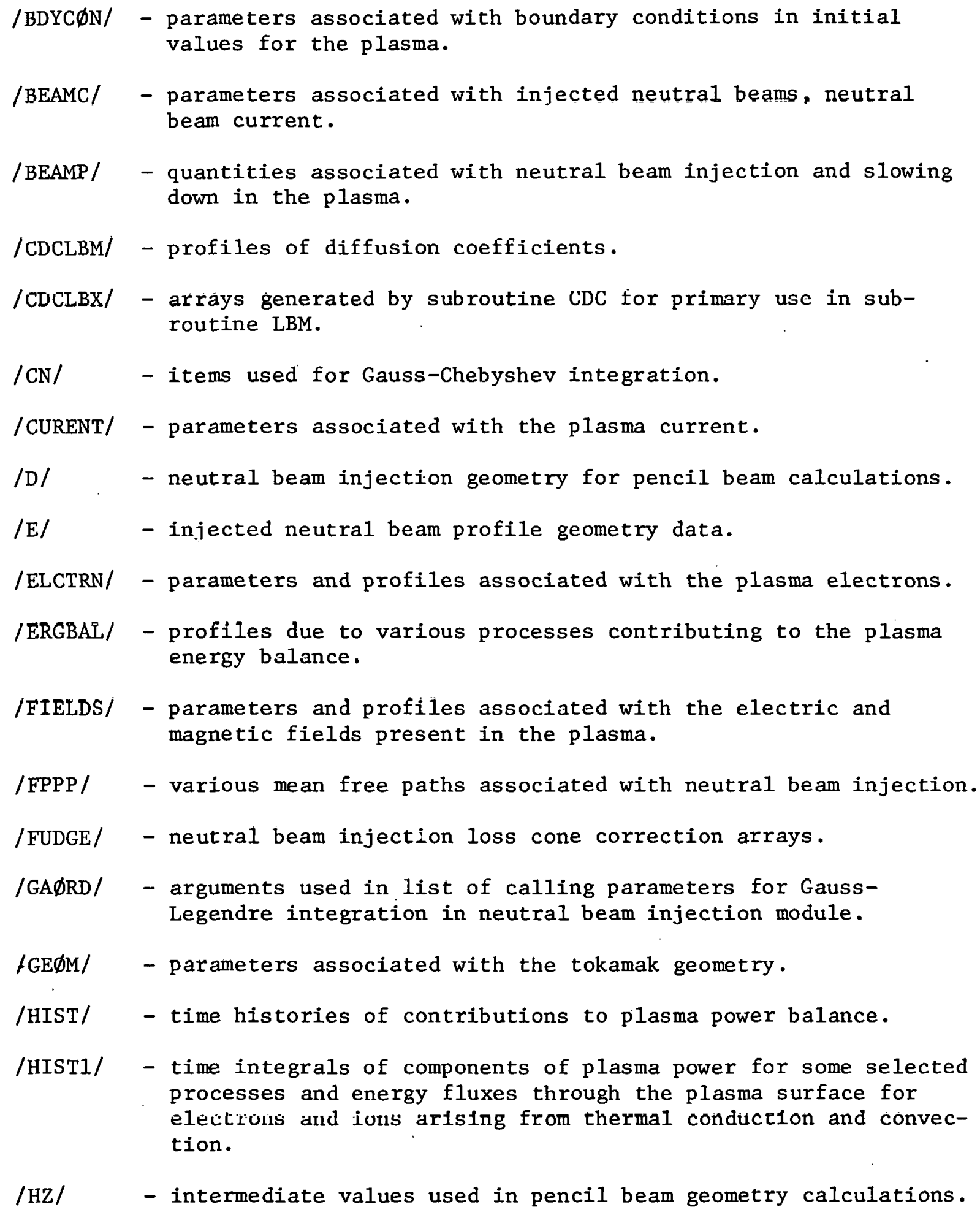

/HZ/ - intermediate values used in pencil beam geometry calculations. 
/IBTMN/ - the solution vector for the set of differenced equations describing the model of the basic plasma.

/MMPURT/ - parameters and profiles associated with the impurities module.

/INDEX/ - switch arrays, number of spatial, time, and printout intervals in a simulation run.

/INPUT/ - input variables for Fokker-Planck moments calculations.

/INTRP/ - set of variables and arrays used for linear interpolation in the neutral beam injection module.

/INTRPS/ - parameters and switch governing type of interpolation used and switch for level of diagnostic printout for the neutral gas detailed slab model calculation.

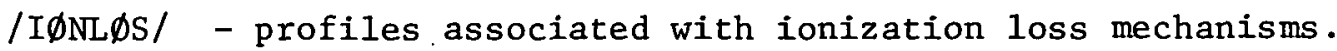

/IøNS/ - parameters and profiles of quantities associated with dominant ions in the plasma (protons).

/JTH/ - total neutral gas attenuation coefficient profile array, $\operatorname{EATN}(101)$.

/LBMIBT/ - coefficient matrix storage array which is loaded in LBM and used by IBTM to generate the solution vectors for the set of finite differenced, coupled partial differential equations describing the basic plasma.

/MEASUR/ - quantities calculated specifically for making comparisons with measurements using microwave interferometer and any other device for which a special calculation must be made.

/NEUTPT/ - pointers used to partition the array WSC.

/NEUTRL/ - parameters and profiles associated with the neutral gas model module.

/NEWS/ - summary of selected information about the evolution of the plasma throughout the simulation.

/NUTRLP/ - parameters, profiles, and switches used tn the neutral gas slab model calculations.

/OUTPT/ - variables and profiles used primarily for purposes of providing output.

/ØUTPUT/ - output variables for Fokker-Planck moments calculations. 
/PINT/ - profiles of power contributions due to the dominant modes involved in the power balance. These profiles are integrals from the plasma center out to the successive radial points in the spatial mesh.

/PøWER/ - profiles due to various processes contributing to the plasma power balance.

/PRNT/ - print control switches associated with the neutral gas module.

/QT/ - source term arrays for basic two-fluid model of the plasma.

/RADLøS/ - profiles of power loss through electromagnetic radiation.

/RATIØS/ - ratios of the various plasma power contributions to the ohmic heating power or total (ohmle + neutral beam injection) input power to the plasma and other assorted quantities.

/RATI $1 /$ - ratios of the dominant plasma power contributions to the total (ohmic + neutral beam injection) input power to the plasma.

/SLAB/ - some variables used in the slab model treatment of neutral gas effects.

/SUMS/ - values of integrals obtained in the process of determining the plasma power balance.

/SURF/ - densities of various chemical elements at the plasma boundary.

/TA/ - injected neutral beam profiles and integration mesh size values.

/TEMP/ - ion, electron, and neutral gas temperature prof1les, plasma ion velocity.

/THRMDF/ - profiles of thermal diffusion coefficients.

/TIME/ - time information for the simulation run exclusive of timing information required by the neutral beam injection module and the impurities module.

/TMINDX/ - timing information required by neutral beam injection module.

/TST $\emptyset$ / - ion and electron temperature profiles stored from the previous time step.

/XPøRT/ - parameters and profiles related to diffusion and thermal transport properties of the plasma.

/XTRA/ - some miscellaneous profiles needed in several subroutines.

/ZDFPS/ - diffusion coefficients for impurity diffusion calculations for the Pfirsch-Ṣchliuter regime. 
II. 5

/ZRAD/ - high- and low-Z profiles of radiation loss due to impurities. 
B. Identification of Variables and Arrays in Labeled Common Blocks

\section{CØMM $\varnothing \mathrm{N} / \mathrm{BDYC} \emptyset \mathrm{N} /$}

\begin{tabular}{|c|c|}
\hline DENB & - electron density at the plasma boundary, $\mathrm{cm}^{-3}$. \\
\hline DEN $\emptyset$ & - initia1 value of electron density at the plasma center, $\mathrm{cm}^{-3}$. \\
\hline DN $\varnothing$ & $\begin{array}{l}\text { - at one time, density of particles added by the neutral beam, } \\
\mathrm{cm}^{-3} \text {. No longer used. }\end{array}$ \\
\hline TEB & - initial value of electron temperature at plasma cenles, eV. \\
\hline TTR & - initial value of ion temperature at plasma center, eV. \\
\hline TEø & - temperature for electrons at the plasma boundary, eV. \\
\hline $\operatorname{TI} \emptyset$ & - boundary and initial temperature of ions. \\
\hline $\mathrm{XI}(10)$ & $\begin{array}{l}\text { - array of exponents for initial plasma profiles. } \\
\text { XI(1), of current density } \\
\text { XI(2), of electron temperature } \\
\text { XI(3), of ion temperature } \\
\text { XI(4), of electron density }\end{array}$ \\
\hline
\end{tabular}


$\underline{\mathrm{C} \emptyset \mathrm{MM} \emptyset \mathrm{N} / \mathrm{BEAMC} /}$

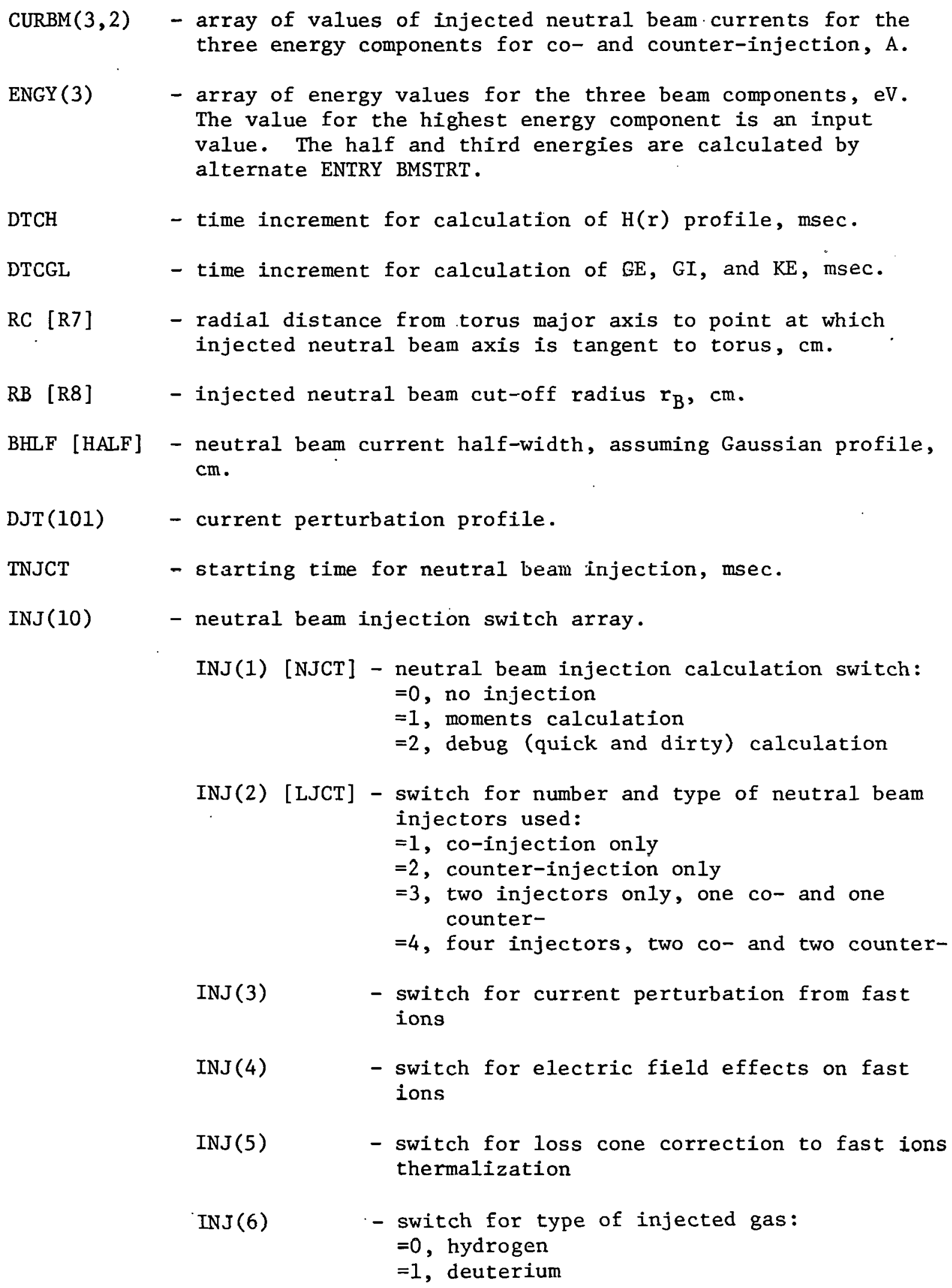
The value for the highest energy component is an input value. The half and third energies are calculated by alternate ENTRY BMSTRT.

DTCH - time increment for calculation of $H(r)$ profile, msec.

DTCGL - time increment for calculation of GE, GI, and $\mathrm{KE}$, msec.

RC [R7] - radial distance from torus major axis to point at which injected neutral beam axis is tangent to torus, $\mathrm{cm}$.

$R B$ [R8] - injected neutral beam cut-off radius $r_{B}$, cm.

BHLF [HALF] - neutral beam current half-width, assuming Gaussian profile, $\mathrm{cm}$.

DJT(101) - current perturbation profile.

TNJCT - starting time for neutral beam injection, msec.

INJ(10) - neutral beam injection switch array.

INJ(1) [NJCT] - neutral beam injection calculation switch: $=0$, no injection

$=I$, moments calculation

$=2$, debug (quick and dirty) calculation

INJ(2) [LJCT] - switch for number and type of neutral beam injectors used:

$=1$, co-injection on $1 \mathrm{y}$

$=2$, counter-injection only

$=3$, two injectors only, one co- and one counter-

$=4$, four injectors, two co- and two counter-

INJ(3) - switch for current perturbation from fast ions

INJ(4) - switch for electric field effects on fast ions

INJ(5) - switch for loss cone correction to fast ions thermalization

INJ(6) - switch for type of injected gas:

$=0$, hydrogen

$=1$, deuterium 
Used primarily with the neutral beam injection module. The following four arrays contain profiles for the three energy components of the neutral beam and the two injection directions, co- and counter-.

$\operatorname{HTMP}(11,3 ; 2)-\mathrm{H}(\mathrm{r})$ profiles.

$\operatorname{GETMP}(11,3,2)$ - profiles of the fraction of beam power transferred to plasma electrons.

$\operatorname{GITMP}(11,3,2)$ - profiles of the fraction of beam power transferred to plasma Ions.

$\operatorname{DJTMP}(11,3,2)$ - current perturbation profiles.

The following three arrays contain temporary profiles.

QETMP (11) - injected neutral beam heat input to electrons.

QITMP(11) - injected neutral beam heat input to ions.

$\operatorname{DJTTMP}(11) \quad$ - current perturbation profile.

PATH(3) - no longer used. Originally had mean free path values for use in moments calculation.

TCGS(2) - pair of time values defining time interval over which $H(r)$ profiles and results of moments calculation are intcrpolated. Array element TCGS(1) contains the larger time valuc.

$\operatorname{SDT}(3,4) \quad$ - array of slowing-down times computed from the moments calculation for each beam energy component.

$\operatorname{SDT}(J, 1) \quad=$ TAVE, average lifetime for slowing down of fast ion

$\operatorname{SDT}(J, 2) \quad=\operatorname{TCXV} \emptyset \mathrm{P}$, average charge-exchange lifetime

$\operatorname{SDT}(\mathrm{J}, 3)=\mathrm{TS} \emptyset \mathrm{PP}$, initial slowing-down time of fast ions

$\operatorname{SDT}(J, 4)=\mathrm{TF} \emptyset \mathrm{PP}$, time for complete thermalization of fast ions

ECRS(3) $\quad=E_{c}$, the critical energy, above which power is transferred from neutral beam to electrons and below which power is transferred predominantly to the ions. 
CØMMфN /BEAMP/ (continued)

The following seven arrays contain values of quantities for the three energy components of the neutral beam and the two injection directions, co- and counter-.

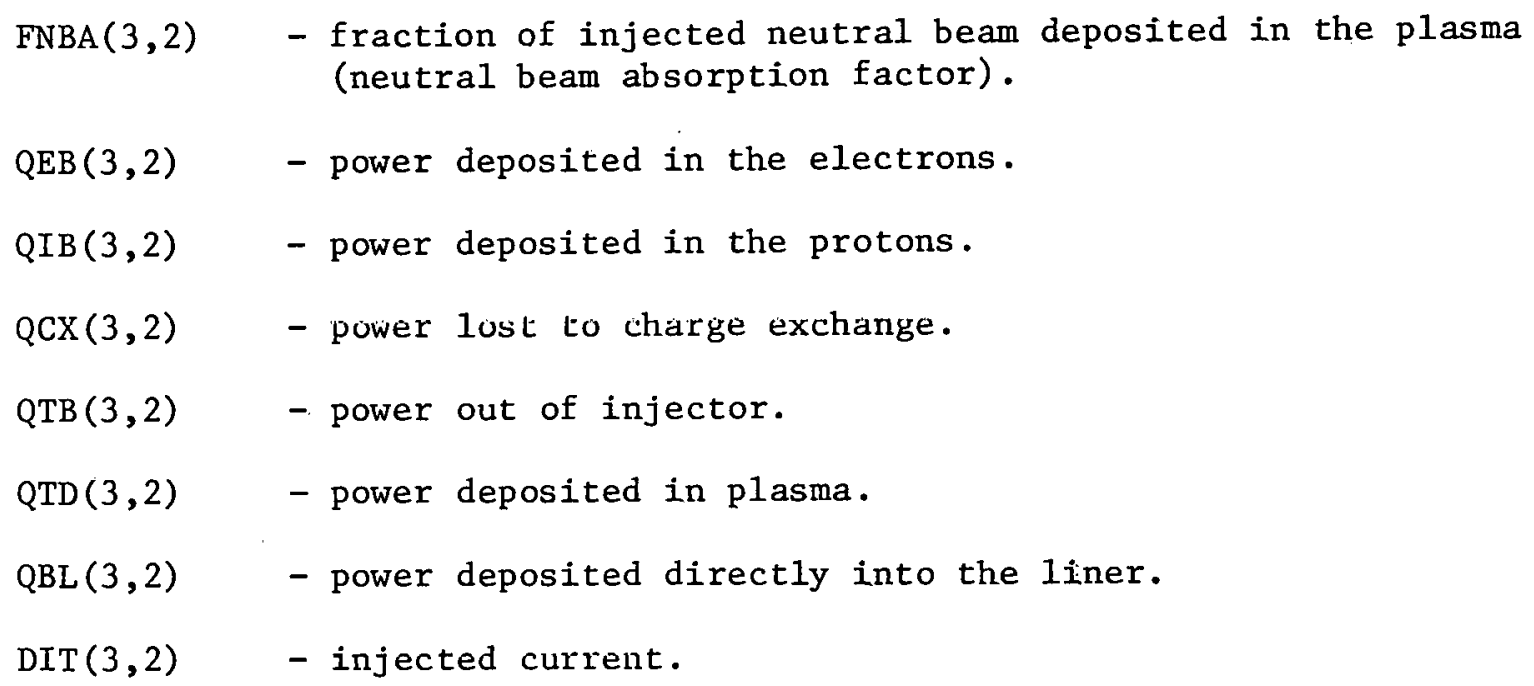

The following seven arrays contain values of quantities for the three energy components of the neutral beam for the sum of the two injection directions.

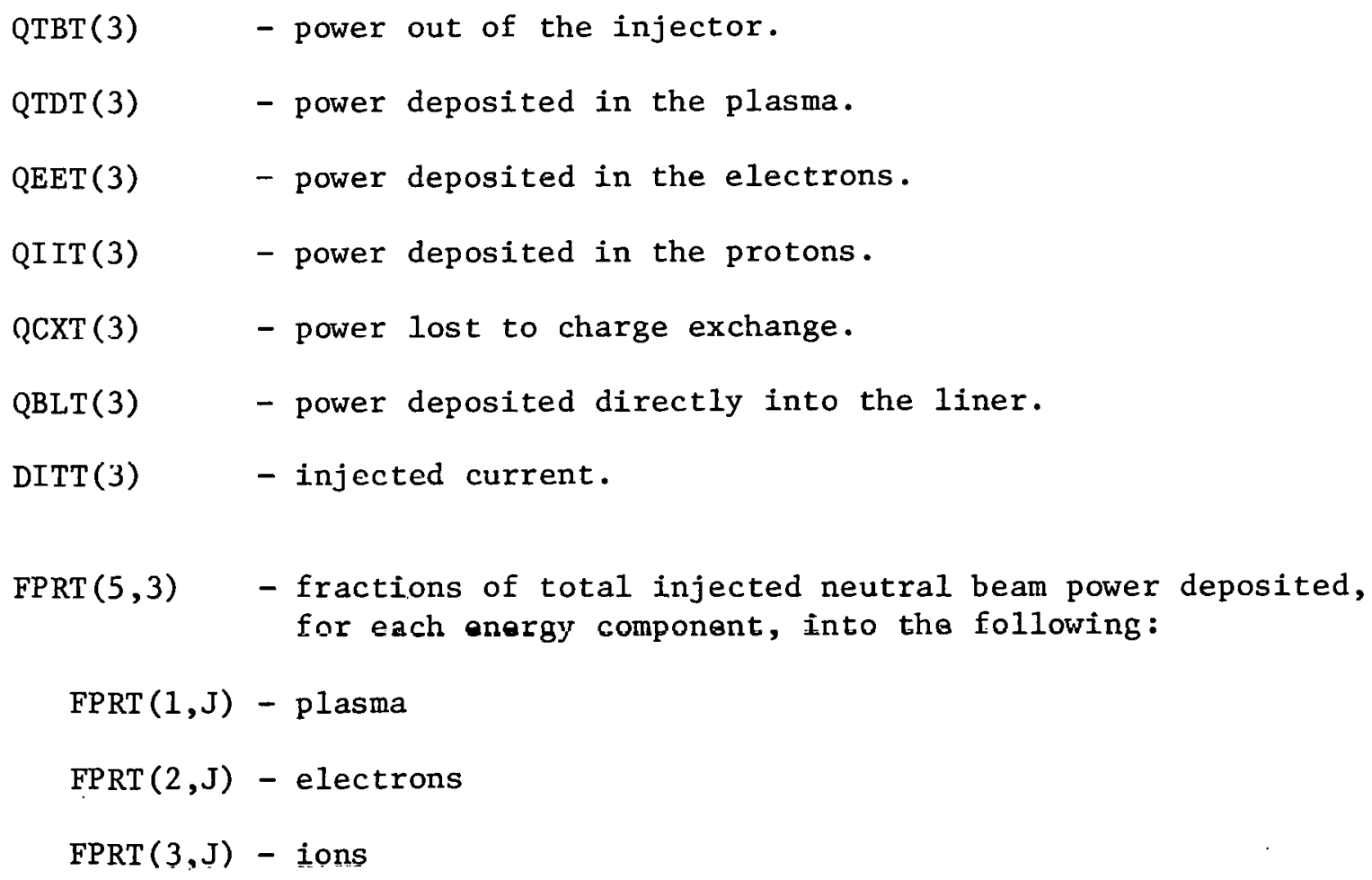


CØMM $\emptyset \mathrm{N} / \mathrm{BEAMP} /$ (continued)

\author{
$\operatorname{FPRT}(4, \mathrm{~J})$ - lost to charge exchange \\ FPRT $(5, J)$ - deposited directly into the liner \\ $\operatorname{DJSUM}(11,2)$ - current density profiles, the sums of the profiles of the \\ three energy components, for the co- and counter-injected \\ beams. \\ PNUM - factor for doubling the number of injectors when computing \\ the vartous contributiuns lo llie puwer Lalaniee. \\ TCHS - time at which $H(r)$ profile is calculated; used for print- \\ UUL. \\ XPRT(3) - stagnation distances $x_{S}$ used in the $H(r)$ calculation for \\ the three beam energy components. \\ MM - subscript variable used with loss cone matrices to dis- \\ tinguish high- and low-Z impurity cases. \\ $=1$ for $10 w-Z$ \\ $=2$ for high $-\mathrm{Z}, \mathrm{DI}(1)>10.0$
}


CØMM $\emptyset \mathrm{N} / \mathrm{CDCLBM} /$

$\begin{aligned} & \operatorname{EE}(101)= \begin{array}{l}\left(B / x_{e}\right)\left(\partial x_{e} / \partial B\right), \text { where } x_{e} \text { is the electron thermal conduc- } \\ \text { tivity. }\end{array}=\left(B / x_{i}\right)\left(\partial x_{i} / \partial B\right), \text { where } x_{i} \text { is the ion thermal conductivity. } \\ & \operatorname{EI}(101) \quad \text { no longer used. } \\ & \operatorname{DIV}(101) \quad-\end{aligned}$

The following seven arrays are reciprocals of the values of the profiles listed.

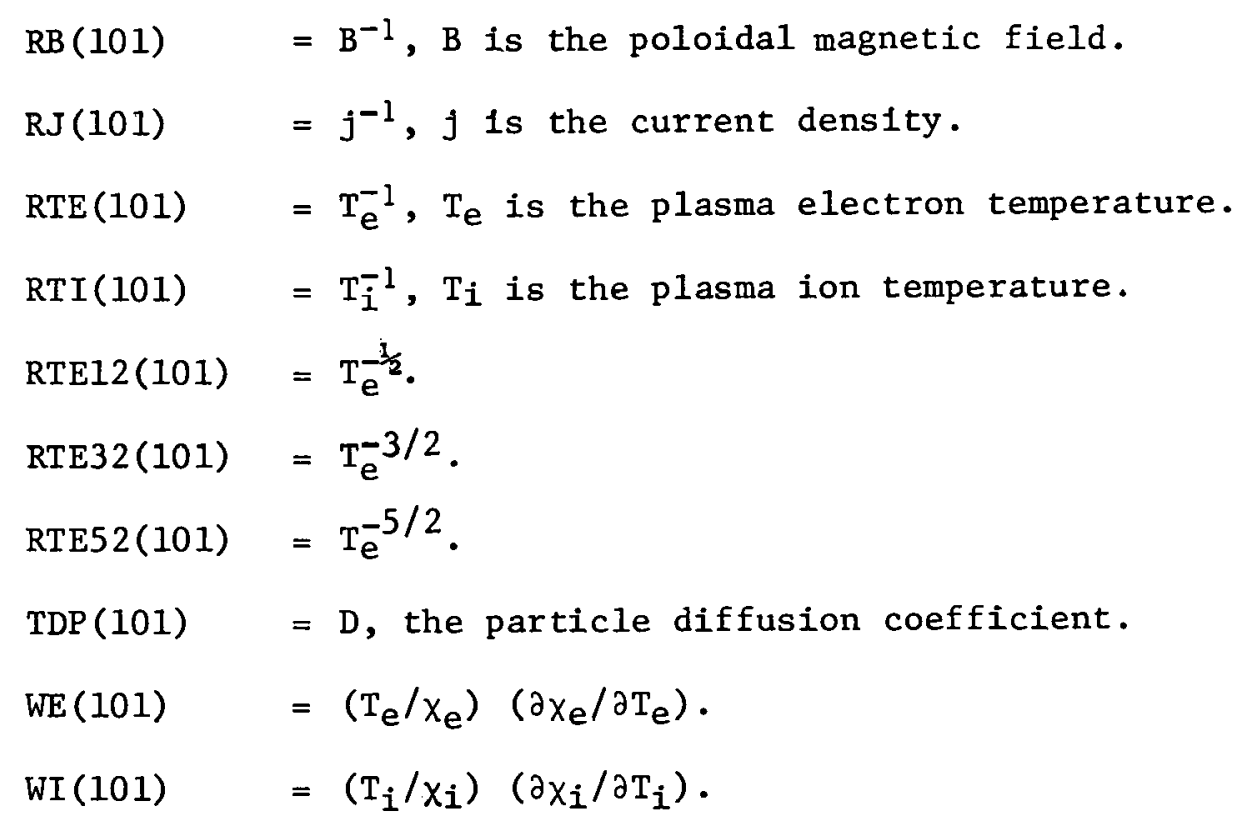


$\underline{\mathrm{C} \emptyset \mathrm{MM} \emptyset \mathrm{N} / \mathrm{CDCLBX}}$

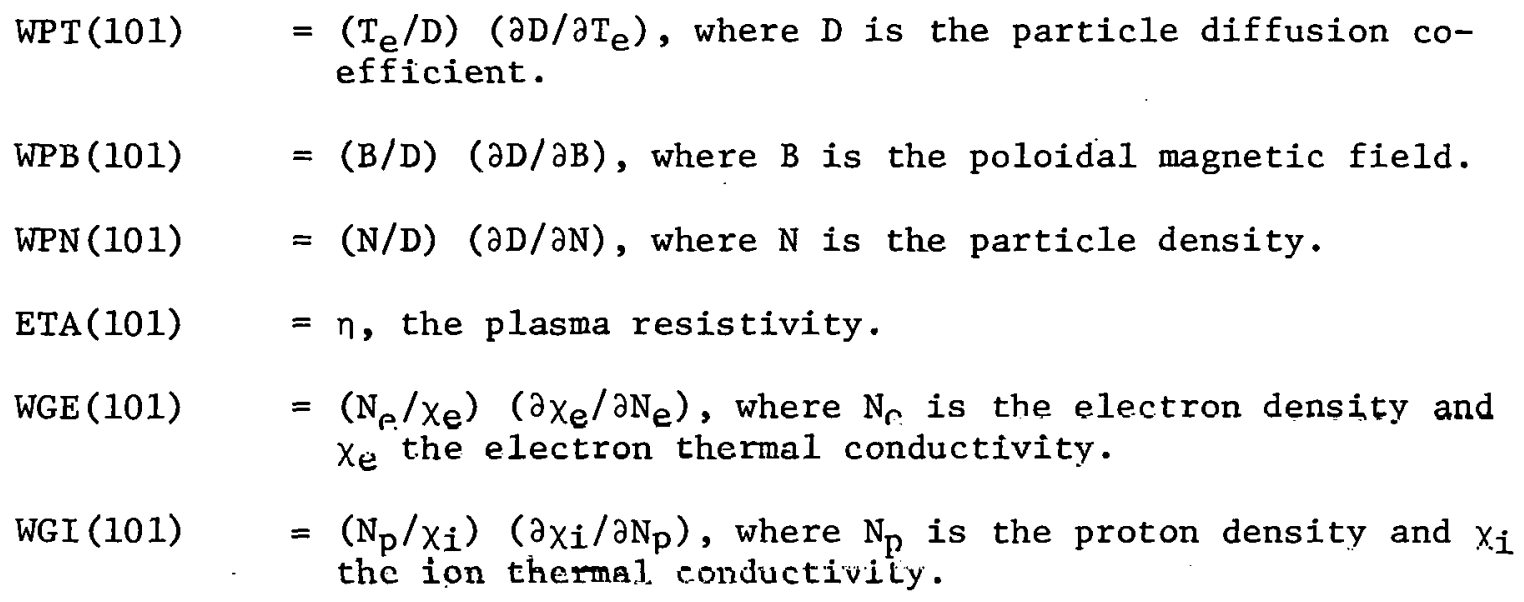


II. 13

CØMM $\emptyset \mathrm{N} / \mathrm{CN} /$

S(15) - array of zeros of the Chebyshev polynomials of degree NP.

S1

- Christoffel numbers $\beta_{j}$ for the Gauss-Chebyshev formula, the coefficient $\pi / n$ for the sum on the right-hand side (see description of subroutine RPT $3 X Y$ in the neutral beam injection module).

NP

- the number of points used by the quadrature formula. 
CØMMфN /CURENT/

ZFI

- additional amount of total current (A) which is added with a characteristic rise time of TC msec.

ZPC

- initial amount of total current (A). This component remains constant throughout the simulated plasma discharge. 
II. 15

CØMM $\emptyset \mathrm{N} / \mathrm{CXL} \emptyset \mathrm{S} /$

QCX(101) - profile of energy loss to ions due to charge exchange.

PCX(101) - profile of power loss to ions due to charge exchange. 


\section{$C \emptyset \mathrm{MM} \emptyset \mathrm{N} / \mathrm{D} /$}

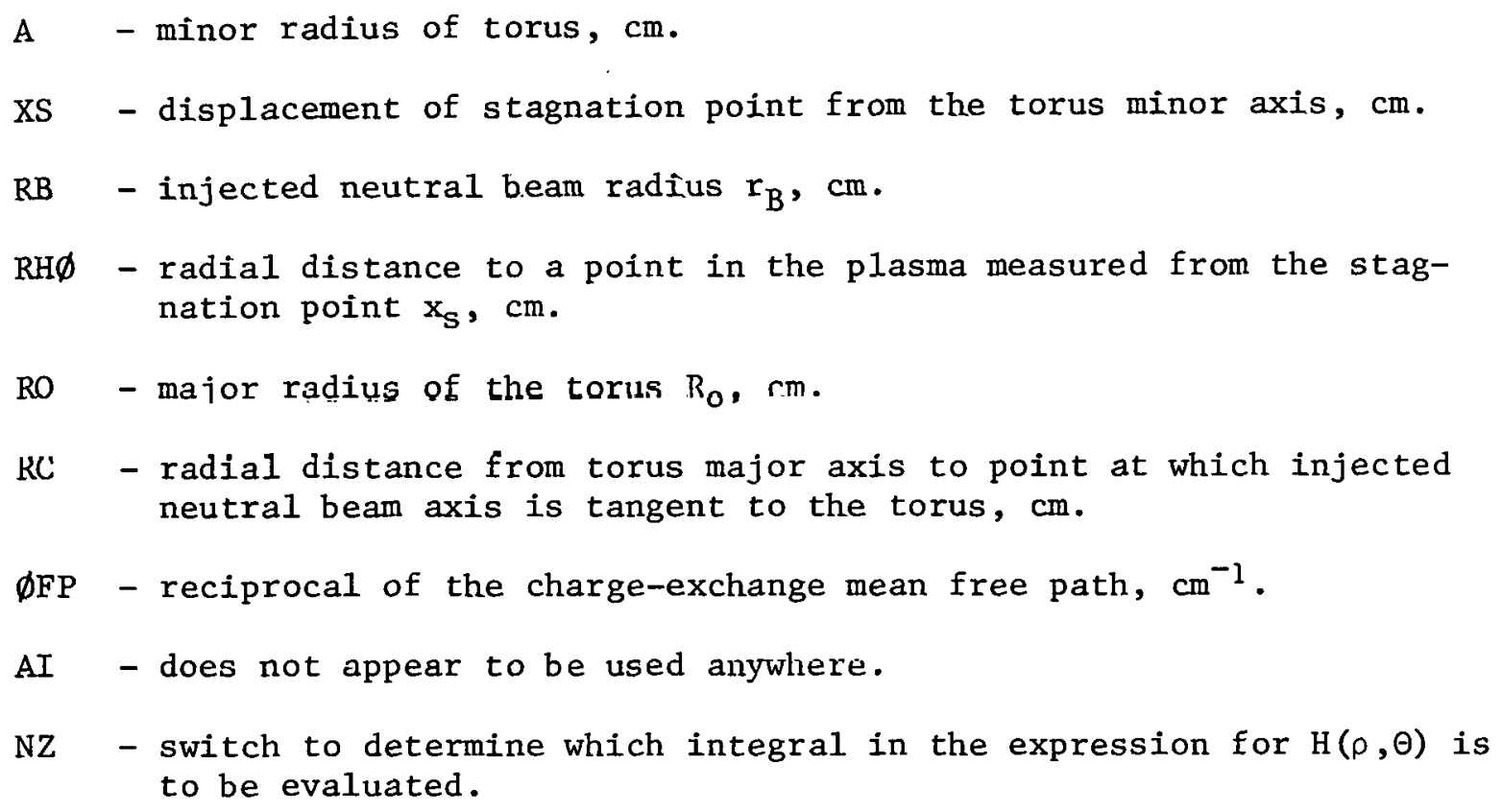


$\underline{C} \emptyset \mathrm{MM} \emptyset \mathrm{N} / \mathrm{E} /$

CRB - current value of $R_{B}$, required by a nested integral, cm.

A5 (A6) - not currently used.

XUP1 - a variable containing one of the limits for the integrals in the pencil beam calculations for neutral beam injection. It has one of the values $R_{O}+x_{S} \pm \sqrt{\rho^{2}-z_{B}^{2}}$ according to whether $\mathrm{NZ}=1,2$.

BEAM - square of the half width of the assumed Gaussian profile shape of the injected neutral beam.

BSHAPE - profile factor for injected neutral beam.

DXX - spacing interval for the XXX array. 
CØMMфN/ELCTRN/

$\mathrm{FQE} \quad$ - fraction of injected neutral beam power transferred to electrons. Used only for debug (quick and dirty) calculation in subroutine BEAM. 
CØMM $\emptyset \mathrm{N} / \mathrm{ERGBAL/}$

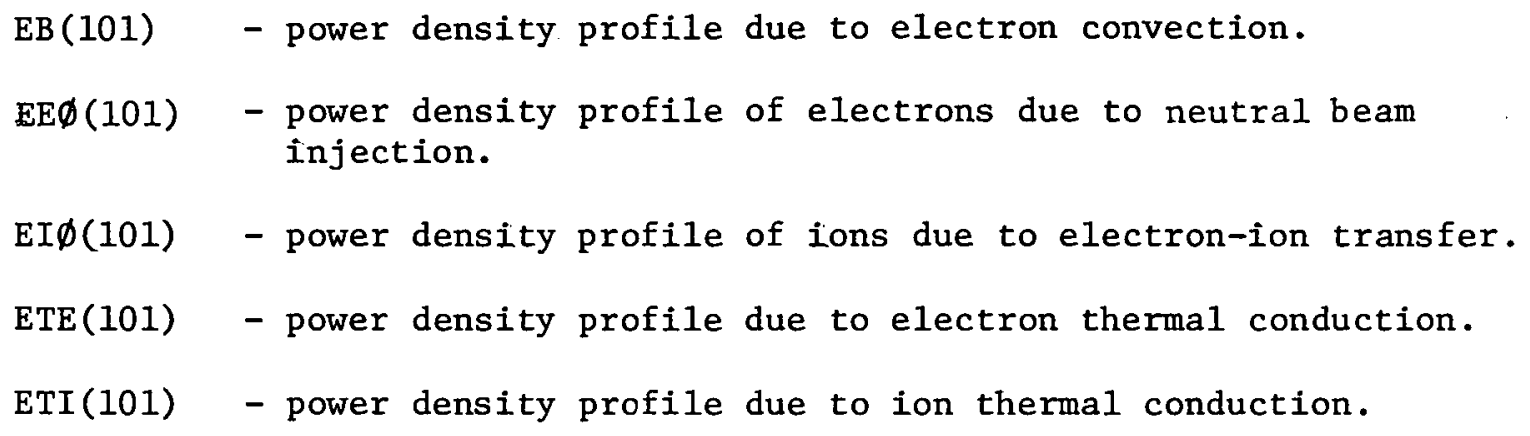


II. 20

\section{CØMMфN /FIELDS/}

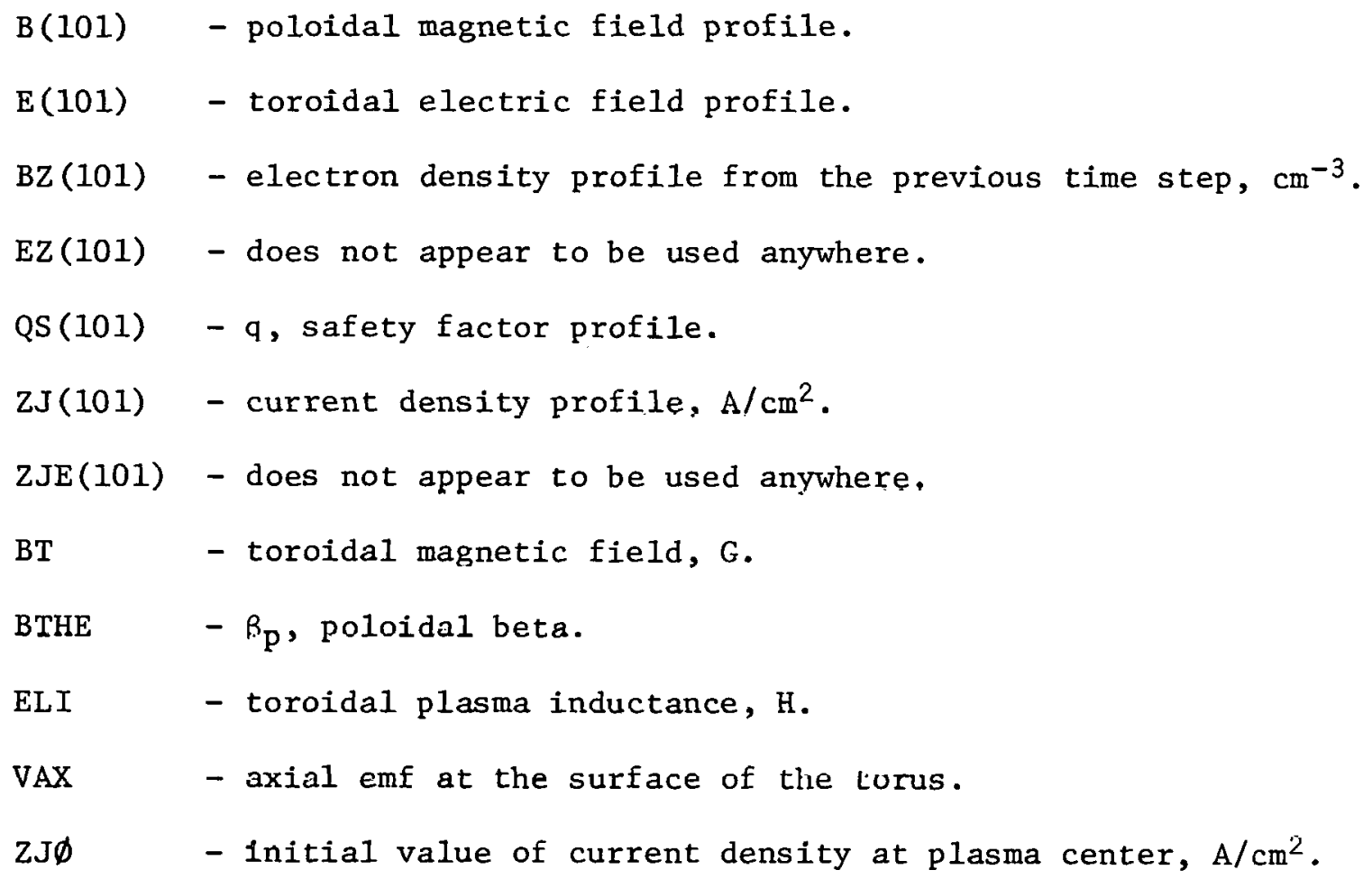


CØMM $\emptyset \mathrm{N} / \mathrm{FPPP} /$

\begin{tabular}{|c|c|}
\hline FPCX & $\begin{array}{l}\text { - mean free path for charge exchange for each neutral beam } \\
\text { energy component, } \mathrm{cm}^{-1} \text {. }\end{array}$ \\
\hline FPI & $\begin{array}{l}\text { - mean free path for impact ionization for protons for each } \\
\text { beam energy component, } \mathrm{cm}^{-1} \text {. }\end{array}$ \\
\hline FPE & $\begin{array}{l}\text { - mean free path for impact ionization for electrons for each } \\
\text { beam energy component, } \mathrm{cm}^{-1} \text {. }\end{array}$ \\
\hline $\mathrm{FP}$ & $\begin{array}{l}\text { - this array is generated in subroutine SUBH, but appears to } \\
\text { serve no purpose. }\end{array}$ \\
\hline FPIMP & - this array also appears to serve no useful purpose. \\
\hline
\end{tabular}


Neutral beam injection loss cone correction arrays.

$\operatorname{ST} 1(10,4,2,2)$ - for power transferred to plasma electrons, no correction.

$\operatorname{ST} 2(10,4,2,2)$ - for power transferred to plasma ions, no correction.

$\mathrm{ST} 3(10,4,2,2)$ - for $\mathrm{KE}$, no corrections.

ST4 $(10,4,2,2)$ - for power transferred to plasma electrons, with corrections.

$\operatorname{ST5}(10,4,2,2)$ - for power transferred to plasma ions, with currections. $\operatorname{ST6}(10,4,2,2)$ - for KE, with corrections. 


\section{$\underline{\mathrm{C} \emptyset \mathrm{MM} \emptyset \mathrm{N} / \mathrm{GA} \emptyset \mathrm{RD} /}$}

NN

- the number of equal subintervals that [XL,XU] is to be divided into. The lower limit of integration is $\mathrm{XL}$, the upper limit XU.

MM

- the number of points (order) at which the integrand is to be evaluated in each subinterval, i.e. total number of evaluations $=\mathrm{NN} * \mathrm{MM}$. Permissible values of $\mathrm{MM}$ are $2,3,4,5,6$, $7,8,9,10,12,16,20,24,32,40,48,64,80$, and 96 . If $\mathrm{MM}<2,2$ points are used. Within the range of $2-96$, if $\mathrm{MM}$ is not one of the permissible values, the next higher permissible value is used. 
II. 24

\section{CØMM $\emptyset \mathrm{N} / \mathrm{GE} \emptyset \mathrm{M} /$}

$\begin{array}{ll}\text { AM } & - \text { torus minor radius, } \mathrm{cm} . \\ \text { AREA } & - \text { total surface area of torus, } \mathrm{cm}^{2} . \\ \mathrm{HR} & - \text { radial element of length, } \mathrm{cm} . \\ \mathrm{R} \emptyset & - \text { torus major radius, } \mathrm{cm} . \\ \mathrm{R} 9 & - \text { plasma minor radius, } \mathrm{cm} . \\ \mathrm{V} \phi \mathrm{L} & - \text { plasma (torus) volume, } \mathrm{cm}^{3} . \\ \mathrm{ZB} & - \text { no longer used. }\end{array}$


CØMMфN/HIST/

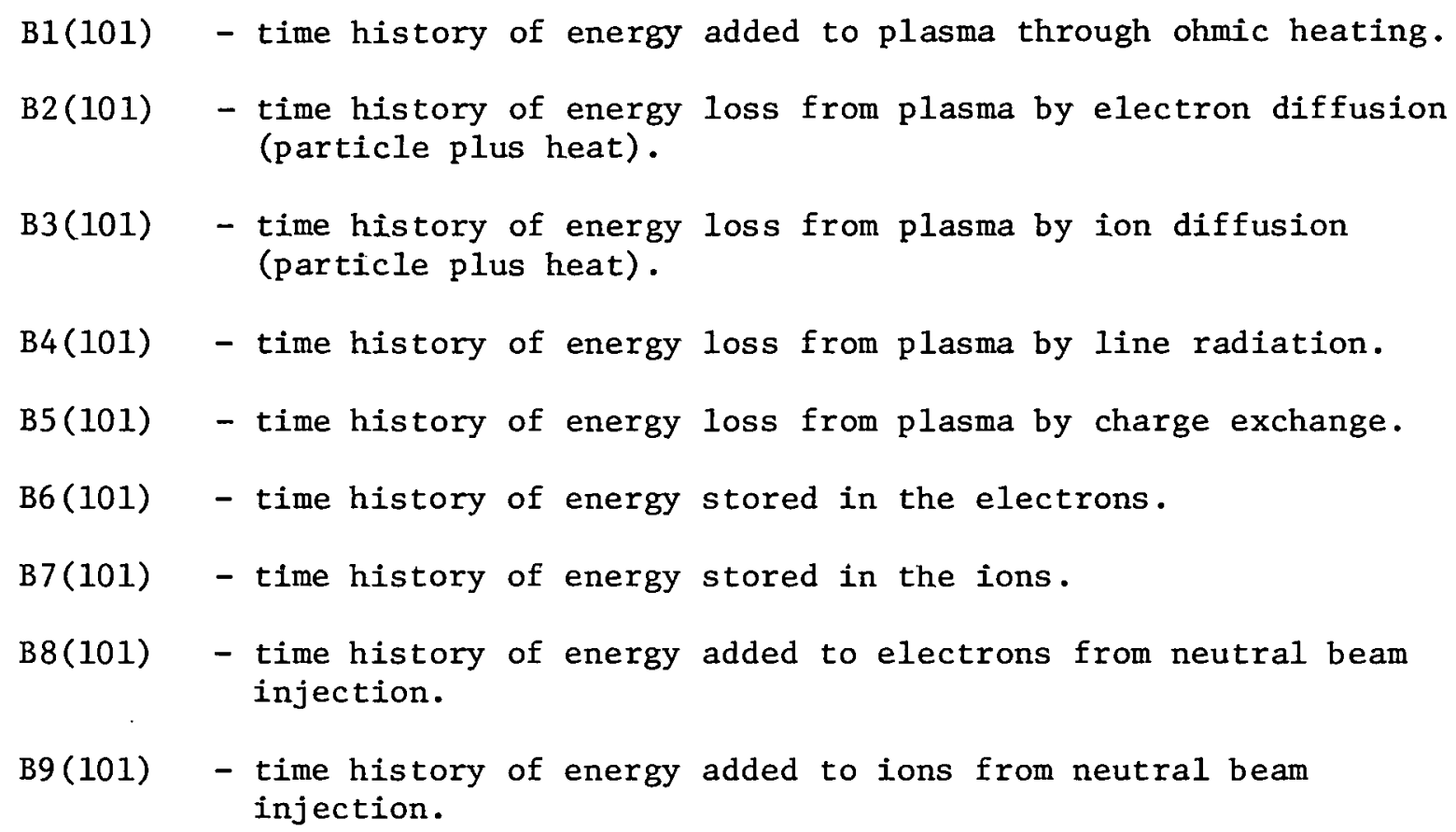


CDMMфN /HIST1/

$P \emptyset X \quad$ - total amount of energy added to plasma by ohmic heating.

PEX - energy loss from plasma due to electron thermal conduction and convection.

PIX - energy loss from plasma due to ion thermal conduction and convection.

PLRX - energy loss from plasma due to line radiation.

PCXX - energy loss from plasma due to charge exchange.

SUMEX - plasma energy stored in the electrons.

SUMIX - plasma energy stored in the ions.

ICM - index variable that is incremented for each new value of time for which a record of the plasma energy balance is desired, used to generate the time history arrays B1-B9.

PHE - energy added to plasma electrons from neutral beam injection.

PHI - energy added to plasma ions from neutral beam injection. 
II. 27

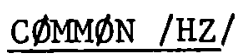

$X Z B,(Z B)$ - a particular value of $z_{B}$, used in a nested integration, $\mathrm{cm}$. 
II. 28

CØMMØN/IBTMN/

$X(360)$ - the solution vector of the plasma profiles, determined by the system of differenced partial differential equations appearing in the plasma module. 
CØMMØN /IMPURT/

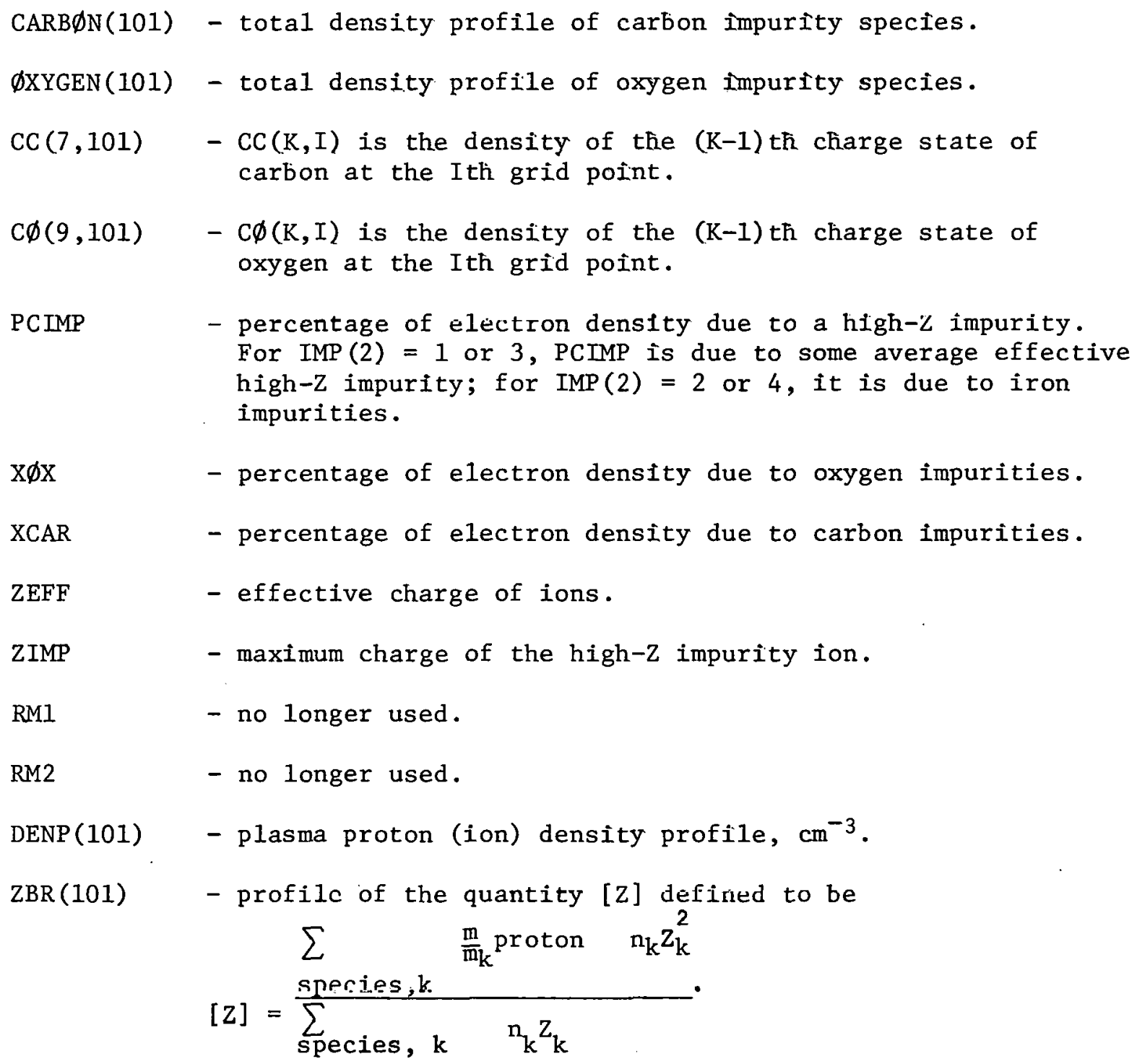

EIMP(101) - power loss profile due to presence of carbon and oxygen impurities. 


\section{CØMM $\emptyset \mathrm{N} /$ INDEX/}

TIC

N

NT

NR

NM1

NUTE (10)

$\operatorname{IMP}(10)$
- basic time interval used for generatlng history arrays in $\mathrm{C} \emptyset \mathrm{MM} \emptyset \mathrm{N} / \mathrm{HIST} /$.

- number of spatial grid points used for radial direction throughout the simulation code.

- total number of time intervals for current pulse and plasma simulation.

- number of times sets of simulated quantitles ublaimed duilig execution of the program are printed.

$=\mathrm{N}-1$, the number of spatial grid intervals in the radial direction.

- switches used to select a simulation path for the neutral gas effects modeling.

NUTE(1), switch for type of computation to be used for the neutral gas simulation:

$=1$, analytic model

$=2$, slab model (Hogan's model, without reflection)

$=3$, for slab model with wall reflections (not yet Included)

$=4$, Monte Carlo transport (NUTRLSN, not yet incliuded)

NUTE(2), exponent in power law for analytic model

- switches used to select a simulation path for modeling impurity atom effects.

IMP(1), switch for initial impurity stripping calculation:

- 1, corono equilibrium table for carhon, oxygen

$=2$, dynamic rate calculation for carbon, oxygen (not an option in current. version of the program)

IMP(2), switch for diffusion of impurities:

$=1$, a corona equilibrium table and radial distribution formulae are used for carbon and oxygen. High-Z impurities are treated using a crude estimate

$=2$, corona equilibrium tables are used for carbon, oxygen, and high-Z Impurity (Fe) atom charge states. Radial distributions are assigned using formulae 
$=3$, a corona equilibrium table together with radial distributions generated by PfirschSchluter diffusion is used for carbon and oxygen. High-Z impurities are treated using a crude estimate

$=4$, corona equilibrium tables are used for carbion, oxygen, and high-z impurity (Fe) atom charge states. Radial distributions are generated by a Pfirsch-Schlüter diffusion mode1

IMP(3), switch for impurity model:

$=1$, use fixed values

$=2$, use model calculation determined by choice of switch value for IMP(2)

IMP(4), switch for detailed model for iron:

$=0$, include iron coronal equilibria

$=1$, omit detailed description

INIT(10) - switches for initiating various options available in the program.

INIT(1), switch for starting mode for initial plasma variable profiles:

$=0$, for profiles generated by analytic functions

$=1$, for profiles read from data cards

INIT (2), not currently used

INIT(3), switch for examining MHD stability characteristics of the plasma:

$=0$, determines the location of the singular surface for $m=3,5$

$=1$, calculates the radial elgenfunctions and tearing mode growth rate according to Furth, Rutherford, and Selberg, for $m=3,5$. Can be used to evaluate transport coefficients in subroutine $\mathrm{CDC}$ 


\section{CØMMØN /INPUT/}

/INPUT/ is used for the moments calculation.

T - elapsed time measured from the time of neutral beam turn-on to the times when the quantities $G_{e}, G_{i}$, and $\Delta J$ are computed.

EO - variable used for temporary storage of one of the three neutral beam energy components. Used in the moments calculation.

AMF - atomic mass of injected fast ions.

AMı - atomic mass of plasma 1ons.

The following six variables are used for temporary storage of quantities at each of the 11 points of the calculational grid elements of the moments calculation.

TEE - electron temperature, eV.

TII - ion temperature, eV.

DNE - electron density, normalized to $10^{13} \mathrm{~cm}^{-3}$.

DNO - total neutral density, normalized to $10^{8} \mathrm{~cm}^{-3}$.

$B R A Z=\langle Z\rangle$ or $Z_{\text {eff }}$.

SQBRZ = [Z].

ZF - fast ion charge.

ETO $=\cos \theta$, the cosine of the neutral beam injection angle.

The following three variables contain values of the upper bounds on error tolerance required by the Simpson's rule integration subroutines used in the moments calculation.

EPA3 - upper bound for ASIMP1.

EPA4 - upper bound for ASIMP2.

EPA5 - upper bound for ASIMP3. 
CØMM $\emptyset \mathrm{N} /$ INPUT/ (continued)

The following three variables contain values of the convergence test selector required by the Simpson's rule integration subroutines.

M3 - convergence test selector for ASIMP1.

M4 - convergence test selector for ASIMP2.

M5 - convergence test selector for ASIMP3.

ICTX - switch variable, used to choose between analytic expression or numerical evaluation of an integral; $\neq 0$, if $\tau_{c x}$ is constant.

LNO - switch variable, used to skip electric field perturbation corrections $(=1)$. 
CØMMØN /INTRP/

$\operatorname{xxx}(101)$ - independent variable array corresponding to the normalized density profile.

PIDEN(101) - plasma denstty profile array, normalized to the central density (see description of subroutine SUBH).

$\mathrm{T} \emptyset \mathrm{L} \quad$ - tolerance factor for test of error size for interpolation procedure.

RTMP(15) - array of normalized (to the maximum radial value associated with the computationd grfd elements of the $H(r)$ calculation In the neutral beam Injection module) radial coordinate values. 
CØMM $\emptyset N$ /INTRPS/

INTRPS - switch for selecting type of interpolation for approximating NI, TI, and EATN.

$=1$, for linear

$=2$, for quadratic

$=3$, for cubic

NSMO - number of division points in the two partition intervals which contain the point of the logarithmic singularity.

NSMO $21=2 *$ NSMO-1.

IDEBUG - diagnostic print switch.

$=0$, for no information

$=1$, prints $A=P 1, B=P 2, C=A F C$, Lipschitz constant, estimate of the number of iterations

$=2$, prints everything listed above plus the profiles NI, TI (or AI), and EATN 
II. 36

$\mathrm{C} \emptyset \mathrm{MM} \emptyset \mathrm{N} / \mathrm{I} \emptyset \mathrm{NL} \emptyset \mathrm{S} /$

QEE(101) - electron power loss due to ionization of neutral hydrogen by electron impact.

QII(101) - proton power loss due to ionization of neutral hydrogen by electron impact.

FF(101) - rate for ionization of neutral hydrogen by electron impact.

SI $\varnothing(101)$ - ionization cross section profile. 
II. 37

$\operatorname{com} \emptyset \mathrm{N} / \mathrm{I} \emptyset \mathrm{NS} /$

$\operatorname{DEN}(101) \quad$ - plasma electron density profile, $\mathrm{cm}^{-3}$.

$\operatorname{DS} \emptyset(101) \quad$ - profile is no longer used.

TES(101) - electron density profile from the previous simulation time step.

AWI

- mass of plasma ions, amu. 


\section{38}

$\mathrm{C} \emptyset \mathrm{MM} \emptyset \mathrm{N} / \mathrm{JTH} /$

EATN(101) - total neutral gas attenuation coefficient profile. 
II. 39

CØMM $\emptyset \mathrm{N} / \mathrm{LBMIBT} /$

A(8008) - matrix of coefficients of set of coupled, differenced partial differential equations describing the plasma evolution in the plasma module. 
$\underline{\mathrm{C} \emptyset \mathrm{MM} \emptyset \mathrm{N} / \mathrm{MEASUR} /}$

FRING - number of microwave fringe shifts with respect to the vacuum due to the presence of plasma electrons.

RVD

- ratio of plasma drift velocity to sound velocity. This variable is not currently used.

XFRI

- value of the integral of the electron density profile. 


\section{CØMM $\varnothing \mathrm{N} / \mathrm{NEUTPT} /$}

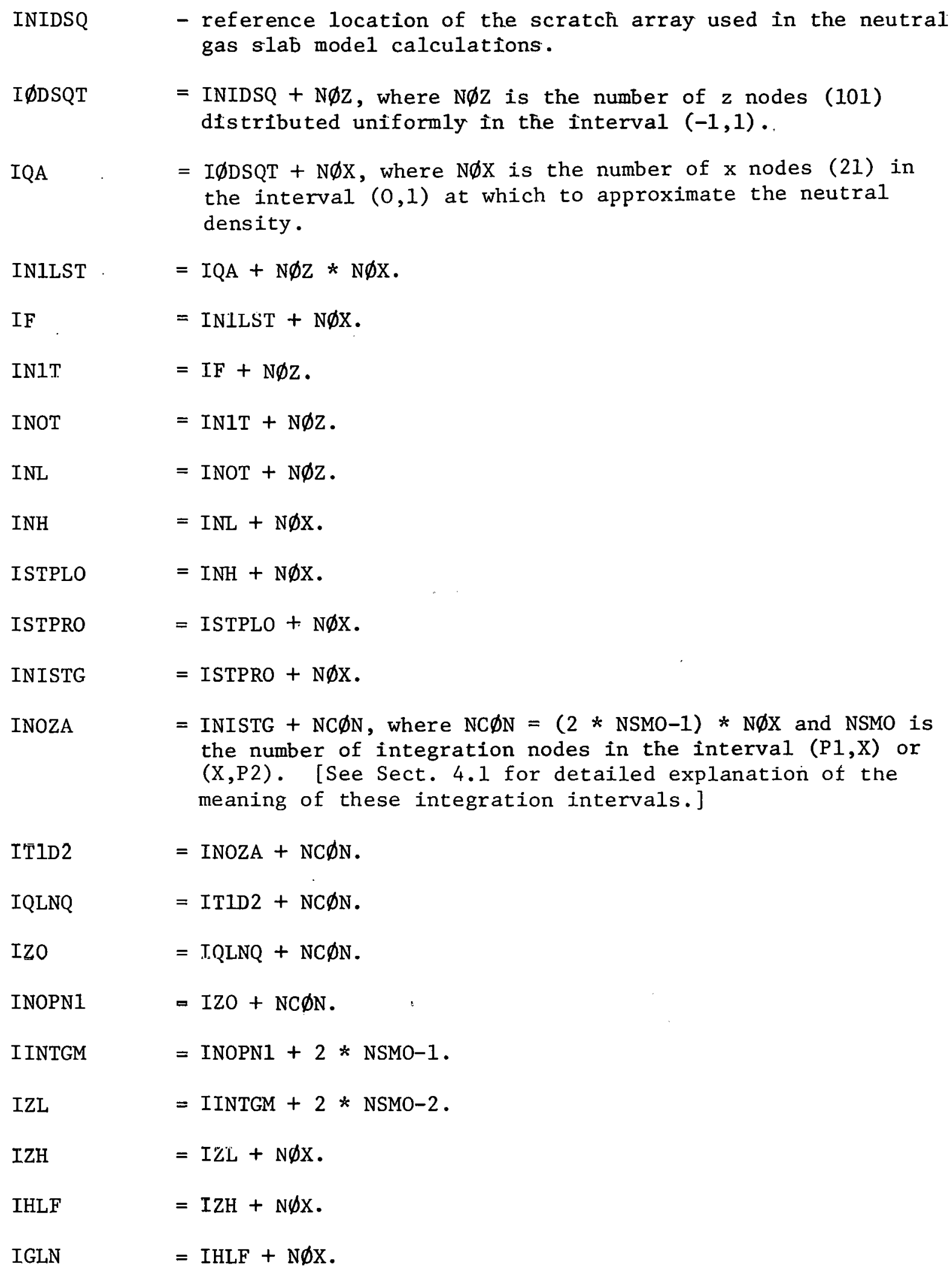




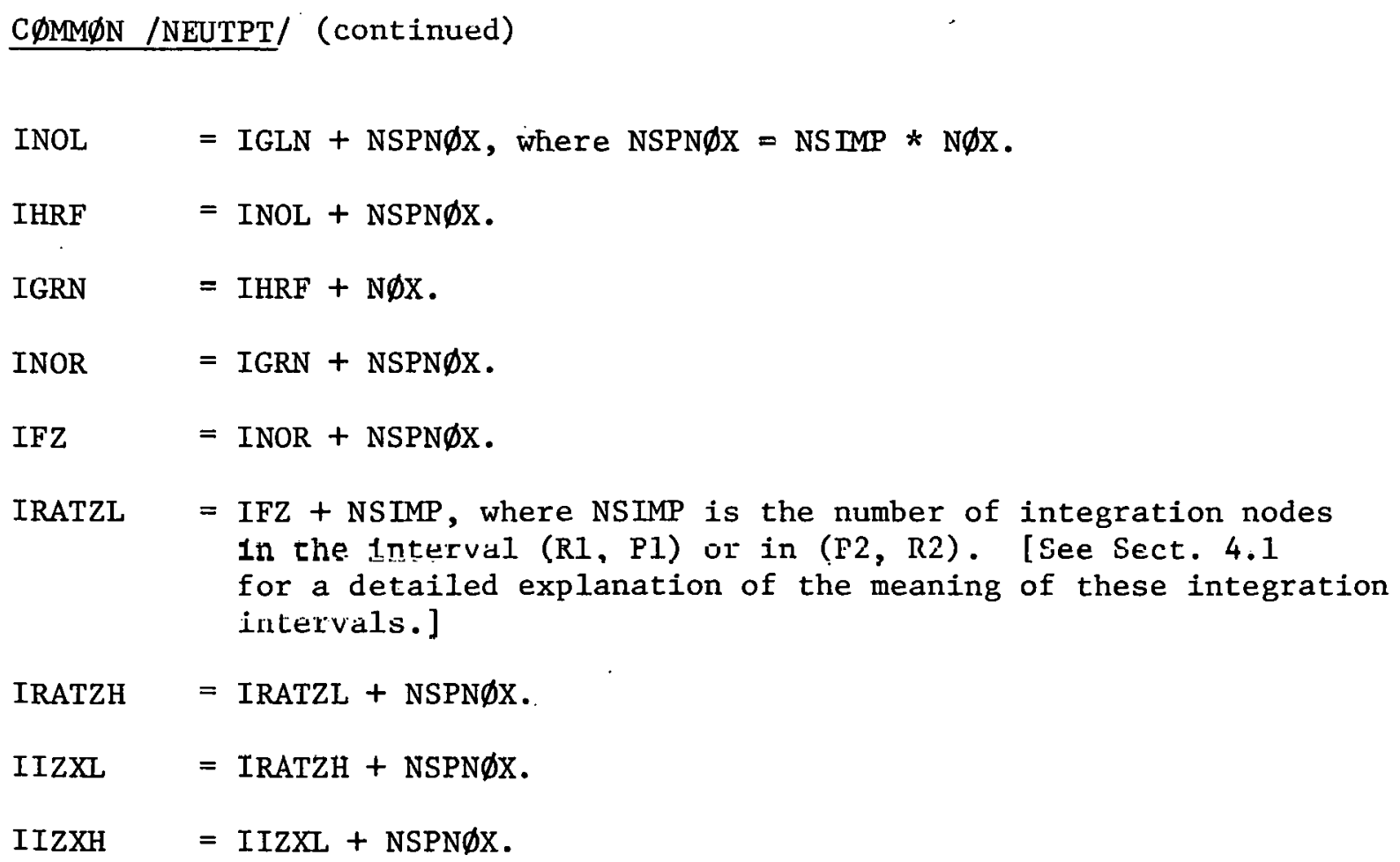


CФMM

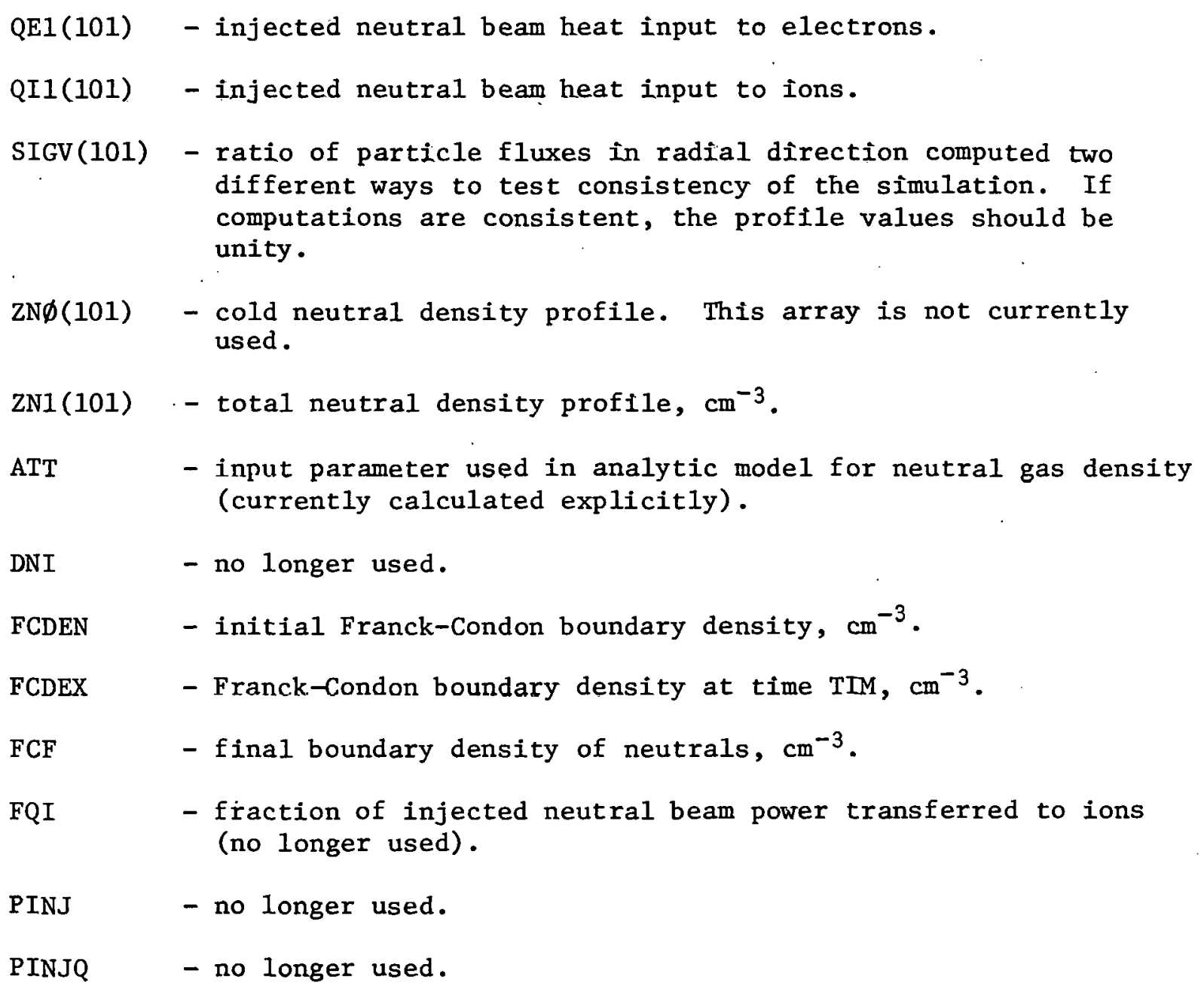


CØMM $\emptyset \mathrm{N} / \mathrm{NEWS} /$

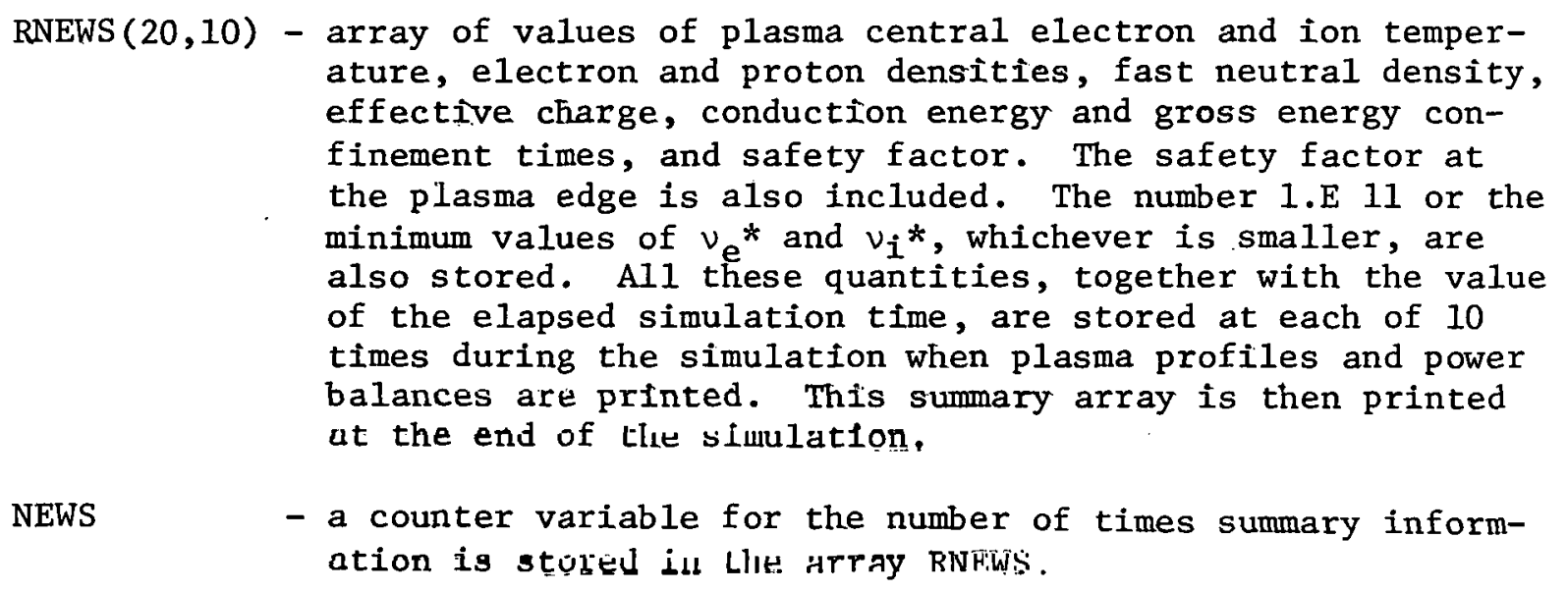


CØMM $\emptyset_{N} /$ NUTRLP/

$\mathrm{N} \emptyset \mathrm{Z}$ - number of uniformly distributed points in the interval $[-1,1]$ used to approximate hot neutral gas density.

$\mathrm{N} \emptyset \mathrm{X} \quad-$ number of points in the interval $[0,1]$ at which to approximate hot neutral gas density in transport calculation.

NS IMP

- number of Simpson nodes used to approximate an integral near a logarithmic singularity in $G(q)$.

NUNIF

- switch for the nodal points in the interval $[0,1]$.

$=0$, points are defined in subroutine NEUTIO to be $(I-1) /$ $(\mathrm{N} \phi \mathrm{X}-1)$

$>0$, points should be placed at the beginning of the array W1 in CØMM $\emptyset \mathrm{N} / \mathrm{NUTRLP} /$

$\mathrm{QF}, \mathrm{QC}$

- determine an integration interval on each side of the logarithmic singularity of $G(q)$ at each nodal point in the interval $[0,1]$. Require $0 \leq \mathrm{QF}<\mathrm{QC}$. Whenever $\mathrm{QF} \Rightarrow \mathrm{QC}$, NEUTIO sets $Q F=0.1 * Q C$.

$\mathrm{DZ}$

$=2 /(\mathrm{N} \emptyset \mathrm{Z}-1)$.

DZD2

$=1 /(N \emptyset Z-1)$.

DZD3

$=2 /(3 *(N \phi Z-1))$.

EPSN1

- convergence criterion for hot neutral gas density solution to the discrete integral equation.

SIMPMI $=$ NS IMP-1.

BDSQTX - - not currently used.

or INO

TCINT

- C* (integral from -1 to 1 of EATN), where

$$
C=\left(1.13 a \sqrt{T_{i}(0)}\right) /\left(\sigma_{c x} N_{i}(0) \sqrt{E \varnothing}\right)
$$

or TIO $=T_{1}(0)$, the fon temperature at the plasma center.

$\mathrm{N} \emptyset \mathrm{XM1}=\mathrm{N} \emptyset \mathrm{X}-1$.

$\mathrm{N} \emptyset \mathrm{XP1}=\mathrm{N} \emptyset \mathrm{X}+1$.

$\mathrm{N} \emptyset \mathrm{ZP1}=\mathrm{N} \emptyset \mathrm{Z}+1$.

NSC

- used as a switch in subroutine NEUTC to bypass some already tabulated values of the convergence function. 
CØMMфN/NUTRLP/ (continued)

ININ

$=0$, pointer for the normalized ion density profile.

ITIN

$=$ ININ $+\mathrm{N}$, pointer for the normalized ion temperature profile.

IN 1

$=$ ITIN+N, pointer for the hot neutral density profile.

IEATN

$=I N 1+N \phi x$, pointer for the estimate of the total neutral gas attenuation coefficient profile.

IINIOX $\quad=$ IINIXI+N, pointer for array of values of the integral

$$
\int_{0}^{y_{I}} d z \operatorname{EATN}(z)
$$

for $I-1, \ldots, N$ and $y_{I}=(I \cdots 1) /(N \cdots 1)$.

IINIXI $=$ IEATN $+N$, pointer for array of values of the integral

$$
\int_{y_{I}}^{1} d z \operatorname{EATN}(z)
$$

for $I=1, \ldots, N$ and $y_{I}=(I-1) /(N-1)$.

IX

- IINIOX $+N$, pointer for array of nodes in $[0,1]$ at which to approximate the neutral density.

IZ

$=I X+N \phi X$, pointer for array of nodes uniformly distributed in $[-1,1]$.

IJL $\quad=\mathrm{IZ}+\mathrm{N} \emptyset \mathrm{Z}$, pointer for the JL array.

IJH $\quad=I J L+N \phi X$, pointer for the $J H$ array.

IIJ $\quad=\mathrm{IJH}+\mathrm{N} \phi \mathrm{X}$, pointer for the IJ array used in the linear interpolation of the cotal neutral density at the nndes 7.(.T).

IRAT = IIJ $+N \emptyset Z$, pointer for the RAT array used in the linear interpolation of the total neutral density $N_{1}$ at the nodes $Z(J)$.

NWDW1 - number of words in the array $W 1$ of the common block /NUTRLP/.

MWDSC - number of words in array A in common block/LBMIBT/.

N

- the number of nodal points in the plasma spatial net (sec also common block /INDEX/).

ANM1 $=$ FLøAT $(\mathrm{N}-1)$.

DSP $\quad=1 . / \mathrm{ANMI}$.

A $\quad=1.13 * A M * \sigma_{C X}(0) N_{i}(0)$.

$\mathrm{B} \quad \mathrm{A}$.

$\mathrm{C} \quad,=\mathrm{A} * \sqrt{\mathrm{T}_{i}(0) / \mathrm{E} \emptyset}$.

WI(1300) - a partitioned array containing the following elements:

$\mathrm{Wl}(\mathrm{ININ}+\mathrm{K})=\mathrm{NI}(\mathrm{K}), \mathrm{K}=1, \ldots, \mathrm{N}$ : normalized ion density

$\mathrm{W} 1(\mathrm{ITIN}+\mathrm{K})=\mathrm{TI}(\mathrm{K}), \mathrm{K}=\mathrm{I}, \ldots, \mathrm{N}$ : normalized ion temperature

$\mathrm{W} 1(\mathrm{IN} 1+\mathrm{I})=\mathrm{N} 1(\mathrm{I})=\mathrm{N} 1(\mathrm{X}(\mathrm{I})), \mathrm{I}=1, \ldots, \mathrm{N} \emptyset \mathrm{X}$

$\mathrm{W} 1($ IEATN $+\mathrm{K})=$ EATN profile approximated at the $\mathrm{K}$-th nodal point, $\mathrm{K}=1, \ldots, \mathrm{N}$ 


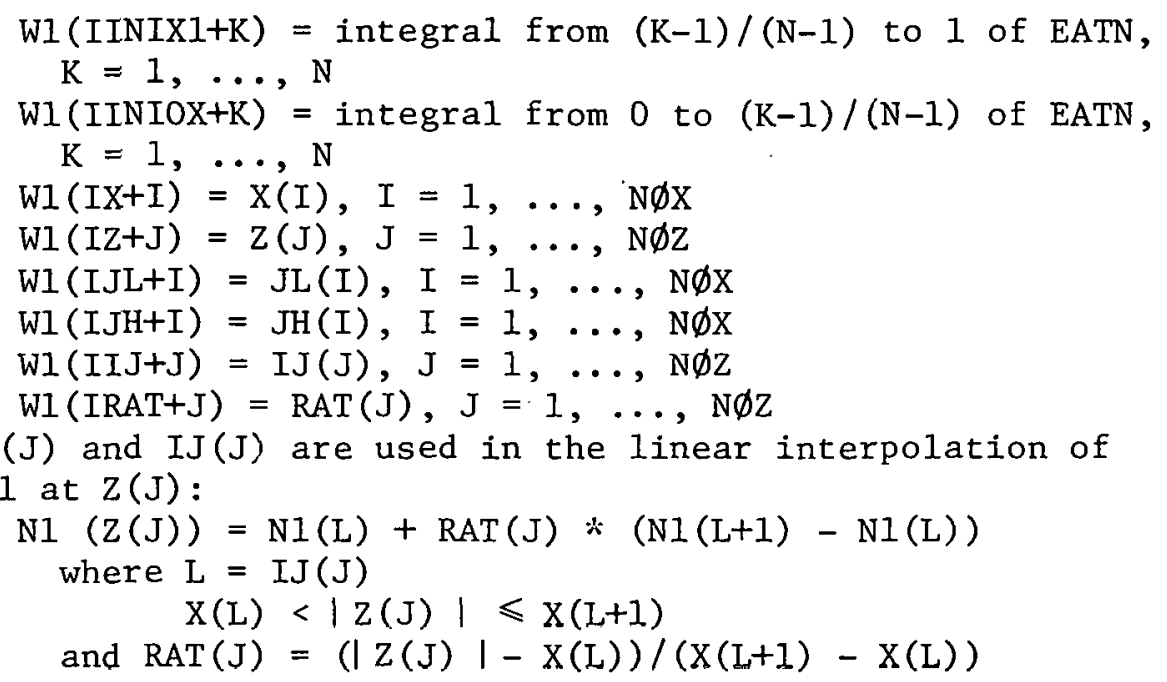


CØMM $\emptyset \mathrm{N} / \emptyset \mathrm{UTPT} /$

$\begin{array}{ll}\text { CTE }(101) & \text { - stored energy, } \mathrm{mJ} / \mathrm{cm}^{3} . \\ \text { TAUE }(101) & \text { - conduction energy confinement time profile, msec. } \\ \text { GAMIN } & \text { - incoming neutral flux. } \\ \text { GAMøUT } & \text { - outgoing flux of charged particles }\left(=\mathrm{N}_{\mathrm{p}} \mathrm{V}\right) . \\ \text { PLR } & \text { - component of plasma power associated with line radiation. }\end{array}$




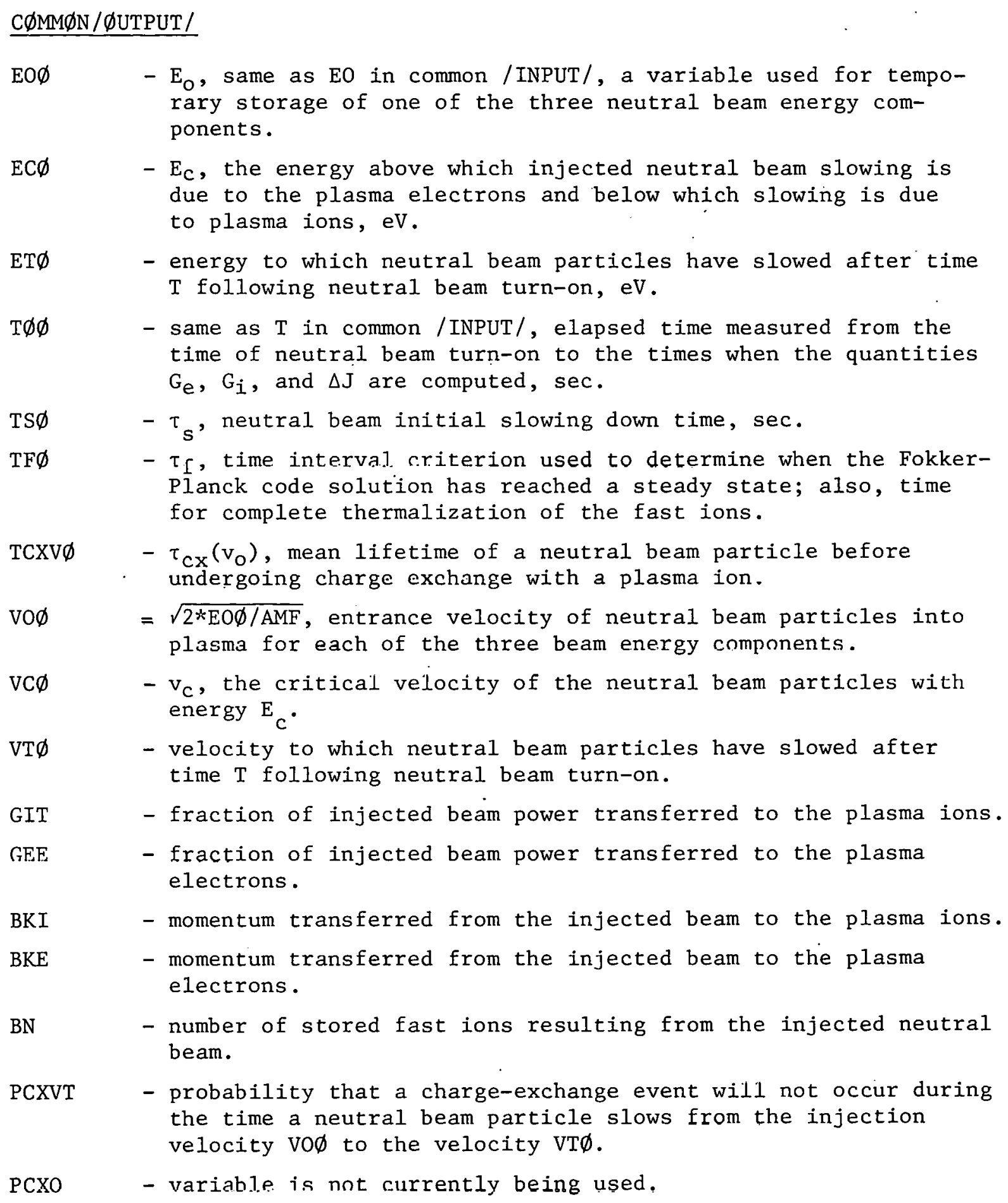


CØMM $\emptyset$ N /

The following seven variables are corrections due to the electric field:

GIE - to fraction of injected neutral beam power transferred to the plasma ions.

GEEE - to fraction of injected neutral beam power transferred to the plasma electrons.

BKIE - to momentum transferred from injected beam to the plasma ions.

BKEE - to momentum transferred from injected beam to the plasma electrons.

BME - to the value of an integral occurring in the moments treatment of the Fokker-Planck equation.

BNE - to the number of stored fast ions. 


\section{$\mathrm{C} \emptyset \mathrm{MM} \emptyset \mathrm{N} / \mathrm{PINT} /$}

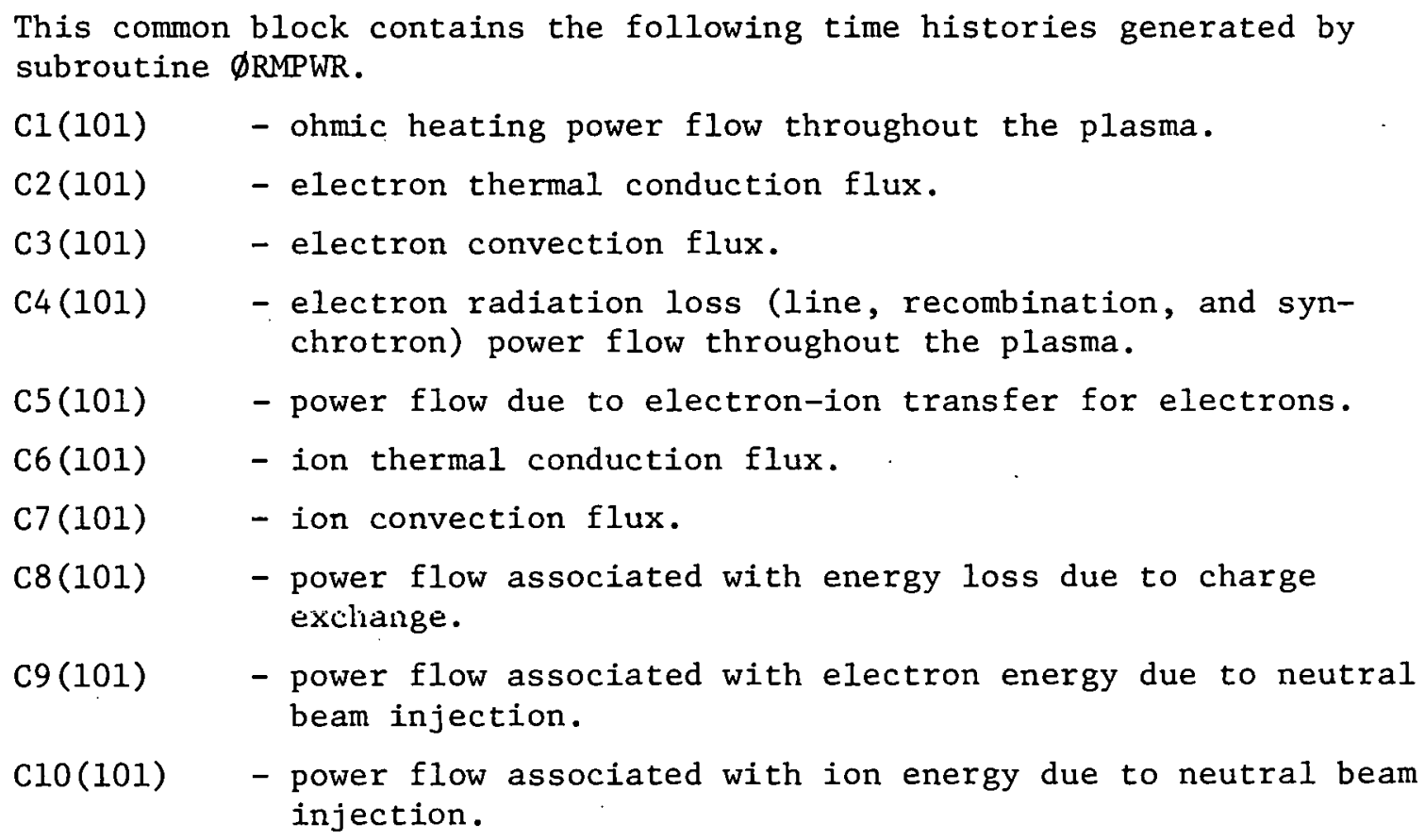




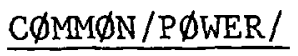

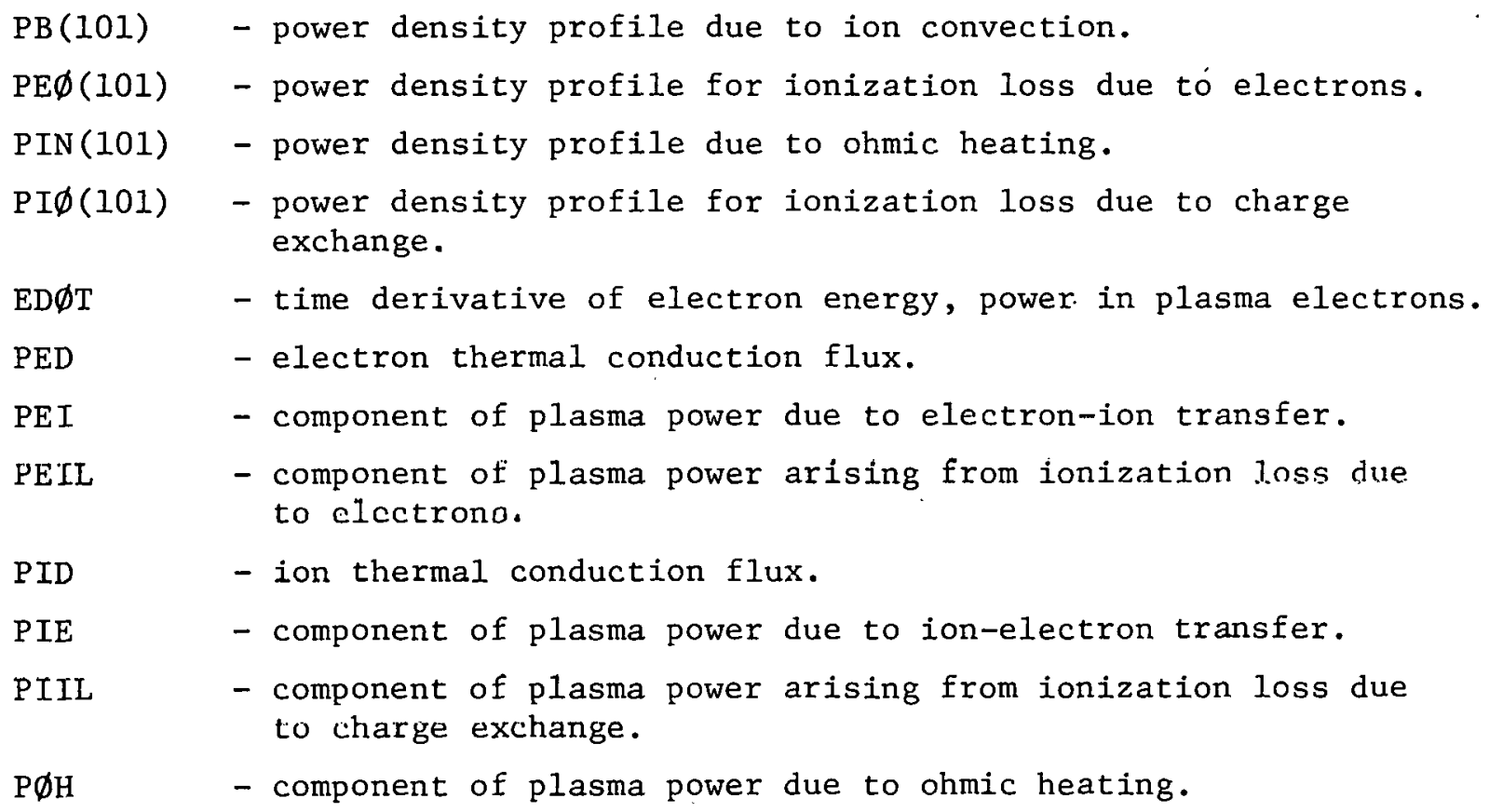


II. 53

$\underline{\mathrm{C} \emptyset \mathrm{MM} \emptyset \mathrm{N} / \mathrm{PRNT} /}$

IPA - not currently used.

IPNO - applicable only if a new slab calculation is to be made $=0$, for no print

$=1$, prints range of relative error curve (see EPSNO)

$=2$, prints same as $=1$ plus old and new NI/AI and neutral density 
II. 54

$\underline{\mathrm{C} Q \mathrm{MM} \emptyset \mathrm{N} / \mathrm{QT} /}$

QE(101) - power gain to the plasma electrons (source term).

QI(101) - power gain to the plasma ions (source term). 


\section{55}

\section{$\underline{\mathrm{C} \emptyset \mathrm{MM} \emptyset \mathrm{N} / \mathrm{RADL} \emptyset \mathrm{S} /}$}
A1 (101) - no longer used.
A2 (101) - no longer used.
A3(101) - rate of change of plasma electron density profile, $\mathrm{cm}^{-3} \cdot \mathrm{sec}^{-1}$.
SHR(101) - power density profile for synchrotron-bremsstrahlung radiation, $\mathrm{kW} / \mathrm{cm}^{3}$.
BREM - not currently used.
SYN - not currently used. 
C $\emptyset \mathrm{MM} \emptyset \mathrm{N} / \mathrm{RATI} \emptyset \mathrm{S}$

$\begin{array}{ll}\text { PCVE } & - \text { power flux from electron convection. } \\ \text { PCVI } & - \text { power flux from ion convection. } \\ \text { RCUR } & - \text { ratio of the plasma current computed by integrating the } \\ & \text { current density to the program current computed by function } \\ & \text { PC. } \\ \text { RCVE } & \text { ratio of power transported by electron convection to the } \\ \text { RCVI } & \text { total power input. } \\ \text { TAX } & \text { same as RCVE, except for ions. } \\ \text { TAXZ } & - \text { gross electron confinement time, msec. } \\ \text { TAXI } & - \text { gross ion confinement time, msec. } \\ & \end{array}$




\section{C $\emptyset \mathrm{MM} \emptyset \mathrm{N} / \mathrm{RATI} \emptyset 1 /$}

This common block contains ratios of power transported by various processes to the total power input (from ohmic heating plus neutral beam injection).

REI

- electron-ion transfer.

RTH

- total thermal conduction.

RPLR

- line radiation.

$\mathrm{RD} \emptyset \mathrm{T}$

- electron heating or cooling.

RTHE

- electron thermal conduction.

RTHI

- ion (proton) convection. 
CØMMON /SLAB/

$\mathrm{E} \emptyset \quad$ - energy of neutrals coming from the wall, eV.

EPSNO - an error upper bound for the magnitude of the relative error between the current value of $(A * N I) / A I$ and the value used previously to get the last basic neutral profile. If this bound is exceeded, then a new slab calculation will be made.

NWDWSC - the number of words in the A array in common block/LBMIBT/. 
II. 59

\section{CøMM $\emptyset \mathrm{N} / \mathrm{SUMS} /$}

SUMBT - energy density associated with the poloidal magnetic field.

SUMC - plasma current obtained by integrating the current density profile.

SUMEI - energy density associated with electron-ion collisions.

SUMLR - energy density due to line radiation.

SUM $\varnothing \quad$ - energy density associated with ohmic heating.

SUMP - energy density associated with electrons.

SUMQE - ionization loss due to electrons.

SUMQI - ionization loss due to charge exchange.

ZE - internal energy of plasma electrons.

ZSI - internal energy of plasma ions.

SNJE - energy in electrons due to neutral beam injection.

SNJI - energy in ions due to neutral beam injection. 
II. 60

\section{CØMMQN / SURF /}

CARN

FENS

ISR $\emptyset \mathrm{N}$
- density of carbon atoms at the edge of the plasma, $\mathrm{cm}^{-3}$.

- density of iron atoms at the edge of the plasma, $\mathrm{cm}^{-3}$.

- logic control variable, not currently used. 
$\underline{\mathrm{C} \emptyset \mathrm{MM} \emptyset \mathrm{N} / \mathrm{TA} /}$

\begin{tabular}{|c|c|}
\hline $\mathrm{HRH} \emptyset(11)$ & - array of values of $H(r, \theta)$, the fast ion production profile. \\
\hline $\mathrm{RV}(11)$ & $\begin{array}{l}\text { - array of radial values at which the fast ion production } \\
\text { profile, } H(r, \theta) \text { before angle averaging, is determined, i.e., } \\
0 \leqslant R V(I)<\text { RH } \emptyset \mathrm{MAX} \text {. }\end{array}$ \\
\hline $\operatorname{HTT}(11)$ & $\begin{array}{l}\text { - value of the integral }\langle H(r, \theta)\rangle_{\theta} \text { at each of the points in } \\
\text { the interval }[0, A M] \text { with grid spacing DELN. }\end{array}$ \\
\hline RHØMAX & $\begin{array}{l}\text { - maximum value of RH } \emptyset \text { permitted, as determined by the posi- } \\
\text { tion of the torus wall or diverter relative to the stagna- } \\
\text { tion point. }\end{array}$ \\
\hline DELH & $\begin{aligned}= & \mathrm{RH} \emptyset \mathrm{MAX} / \mathrm{NCGLM} \text {, radial increment with respect to maximum value } \\
& \text { of } \mathrm{RH} \emptyset, \mathrm{cm} .\end{aligned}$ \\
\hline DELN & $\begin{aligned}= & \text { AM/NCGLM, radial increment with respect to torus minor } \\
& \text { radius } A M, \mathrm{~cm} .\end{aligned}$ \\
\hline NGLP 1 & $\begin{array}{l}\text { - number of computational grid elements plus one, used for } \\
\text { the } H(\rho(\theta)) \text { profile. }\end{array}$ \\
\hline
\end{tabular}


II. 62

\section{CФMM}

TE(101) - plasma electron temperature profile, eV.

TI(101) - plasma ion temperature profile, eV.

$\mathrm{T} \emptyset(101)$ - neutral gas temperature profile, eV.

VEL(101) - plasma ion drift velocity, $\mathrm{cm} / \mathrm{sec}$. 


\section{CØMM $\emptyset \mathrm{N} / \mathrm{THRMDF} /$}

TDE(101) - the (pseudoclassical) electron thermal diffusion coefficient.

$\operatorname{TDI}(101)$ - the (neoclassical) ion thermal diffusion coefficient. 
$\underline{\mathrm{C} \emptyset \mathrm{MM} \emptyset \mathrm{N} / \mathrm{TIME} /}$

HT - current increment for simulation time step, msec.

TC - rise time of the plasma current, msec.

TSTAR - simulation starting time, msec.

TIM - current value of elapsed simulation time, msec.

TMAX - maximum duration of plasma current pulse, msec.

TNJ - not currently used.

TP

TPR - time interval between successive detailed printouts of plasma variable profiles and power balianceo, moec. 
II. 65

$\underline{\mathrm{C} \emptyset \mathrm{MM} \emptyset \mathrm{N} / \mathrm{TMINDX} /}$

DTNJCT - neutral beam injection current rise time, msec.

N1

- number of computational time steps in plasma current rise time interval.

N3

- number of computational time steps in injected neutral beam current rise time interval. 
II. 66

CфMM $\phi \mathrm{N} / \mathrm{TST} \emptyset \mathrm{R} /$

TEL(101) - profile of electron temperature stored from previous time interval.

TIL(101) - profile of ion temperature stored from previous time interval. 
$\underline{\mathrm{C} \emptyset \mathrm{MM} \emptyset \mathrm{N} / \mathrm{XP} \emptyset \mathrm{RT} /}$

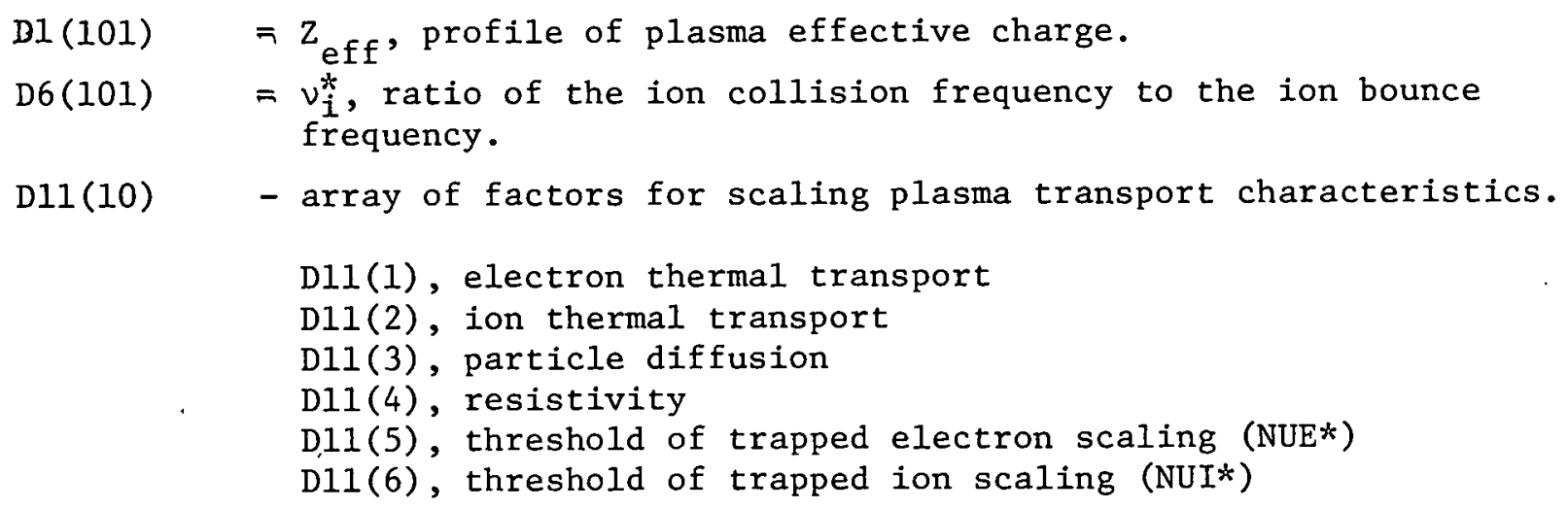




$$
\text { II. } 68
$$

CØMM $\emptyset \mathrm{N} / \mathrm{XTRA} /$
$\operatorname{ALF}(101)=v_{e}^{*}$, ratio of the electron collision frequency to the electron bounce frequency.
$\mathrm{F}(101) \quad-\tau_{\mathrm{p}}$, particle confinement time profile, msec.
$\mathrm{TE12}(101)=\sqrt{\mathrm{T}}$, square root of values of the electron temperature profile. 
II. 69

CØMM $\emptyset \mathrm{N} / \mathrm{ZDFPS} /$

TDZZ(101) - impurity species diffusion coefficient.

ZGRAD(101) - a collection of ion temperature and density gradient terms. 
II. 70

CØMM $\varnothing \mathrm{N} / \mathrm{ZRAD} /$

QRD(101) - low-Z impurity radiation contribution to power $10 \mathrm{ss}, \mathrm{W} / \mathrm{cm}^{3}$.

HIZ (101) - high-Z impurity radiation contribution to power loss, $\mathrm{W} / \mathrm{cm}^{3}$.

DHI(101) - not currently used.

ZHI(101) - not currently used. 
APPENDIX III

III. 1 
Appendix III presents some examples to show how the matrix elements in subroutine LBM are obtained. The $T_{e}$ and $E$ equations (numbers 1 and 5) will be used in the examples.

Consider the electron thermal conduction term in the $\mathrm{T}_{\mathrm{e}}$ equation evaluated at the time step $N+\frac{1}{2}$,

$$
\left.\left.\left.\frac{1}{r_{e}} \frac{\partial}{\partial r}\left(r_{e} x_{e} \frac{\partial T_{e}}{\partial r}\right)\right]^{N+\frac{1}{2}}=x_{e} \frac{\partial^{2} T_{e}}{\partial r^{2}}\right]^{N+\frac{1}{2}}+\frac{1}{r N_{e}} \frac{\partial}{\partial r}\left(r_{e} x_{e}\right) \frac{\partial T e}{\partial r}\right]^{N+\frac{1}{2}} \text {. }
$$

Now let

$$
x \frac{\partial^{2} I^{2}}{\partial \mathrm{r}^{2}}=\frac{X}{(\Delta r)^{2}}\left[\mathrm{I}_{\mathrm{I}+1}=2 \mathrm{I}_{\mathrm{I}}+\mathrm{T}_{\mathrm{I}-1}=\frac{1}{(\Delta \mathrm{r})^{2}} \delta_{1 \mathrm{I}}\right.
$$

and

$$
\frac{1}{\mathrm{rN}_{e}} \frac{\partial}{\partial \mathrm{r}}\left(\mathrm{rN}_{\mathrm{e}} \mathrm{x}_{\mathrm{c}}\right) \frac{\partial \mathrm{T} e}{\partial \mathrm{r}}=\frac{1}{\mathrm{rN}_{\mathrm{e}}}\left[\frac{\mathrm{y}_{\mathrm{I}+1}-\mathrm{y}_{\mathrm{I}-1}}{2 \Delta \mathrm{r}}\right]\left[\frac{\mathrm{T}_{\mathrm{I}+1}-\mathrm{T}_{\mathrm{I}-1}}{2 \Delta \mathrm{r}}\right]
$$

where $y=\operatorname{rN}_{\odot} x_{\theta}$ and

$$
\delta_{2 I}=\frac{1}{4 r N_{e}}\left[y_{I+1}-y_{I-1}\right]\left[T_{I+1}-T_{I-1}\right] \text {. }
$$

The difference representation above can be put in the form of a linear combination of $\mathrm{B}^{\mathrm{N}+1}$ and $\mathrm{T}^{\mathrm{N}+1}$ if the expressions $\delta_{1 . T}^{\mathrm{N}+1}$ and $\delta_{2 . T}^{\mathrm{N}+1}$ are linearized in time:

$$
\begin{aligned}
\delta_{1 I}^{N+1}= & \delta_{1 I}^{N}+\frac{\partial \delta}{\partial t} I^{N} \Delta t+\theta\left[(\Delta t)^{2}\right] \\
\Delta t \frac{\partial \delta}{\partial t} 1 \mathrm{~N}= & \Delta t \frac{\partial}{\partial t}\left[\chi_{I}\left(T_{I+1}-2 T_{I}+T_{I-1}\right)\right] \\
= & \Delta t\left[X_{I} \frac{\partial}{\partial t}\left(T_{I+1}-2 T_{I}+T_{I-1}\right)+\left(T_{I+1}=2 T_{I}+T_{I-1}\right)\left(\frac{\partial x_{I}}{\partial B_{I}} \frac{\partial B_{T}}{\partial t}\right.\right. \\
& \left.\left.+\frac{\partial B_{I}}{\partial T_{I}} \frac{\partial T_{I}}{\partial t}\right)\right] .
\end{aligned}
$$

Now $X=A B^{\theta} T^{\omega}$ so that $\frac{\partial X}{\partial B}=\frac{\theta}{B} X$ and $\frac{\partial X}{\partial T}=\frac{\omega_{X}}{T}$.

Finally, using $\frac{\partial h}{\partial t}=\frac{1}{\Delta t}\left[h^{N+1}-h^{N}\right]$ gives 


$$
\begin{gathered}
\Delta t \frac{\partial \delta_{I T}^{N}}{\partial t}=X_{I}\left\{T_{I+1}^{N+1}-2 T_{I}^{N+1}+{ }_{T}^{N+1}+\left({ }_{T}^{N}{ }_{I+1}^{N}-2 T_{I}^{N}+T_{I-1}^{N}\right)\left[\begin{array}{l}
\theta_{I} \\
B_{I}^{N}
\end{array}\right.\right. \\
+\frac{\omega_{T}^{N}}{T_{I}^{N}}\left({ }^{N+1}-B_{I}^{N}\right) \\
.
\end{gathered}
$$

Similarly,

$$
\begin{aligned}
& \Delta t \frac{\partial \delta_{2}^{N}}{\partial t}=\frac{I}{4 r_{I} N_{I}}\left\{r_{I+1} N_{I+1} x_{I+1}\left[\frac{\theta_{I+1}}{B_{I+1}}\left(B_{I+1}^{N+1}-B_{I+1}^{N}\right)+\frac{\omega}{T}_{I+1}^{\omega}\left(T_{I+1}^{N+1}-T_{I+1}^{N}\right)\right]\right. \\
& \left.-r_{I-1} N_{I-1} X_{I-1}\left[{ }_{B_{I-1}}^{\theta}\left(B_{I-1}^{N+1}-B_{I-1}^{N}\right)+{ }_{T_{I-1}}^{{ }^{N}}\left(T_{I-1}^{N+1}-T_{I-1}^{N}\right)\right]\right\} \\
& \times\left(\mathrm{T}_{\mathrm{I}+1}-\mathrm{T}_{\mathrm{I}-1}\right) \\
& +\frac{1}{4 r_{I}{ }^{N}}\left(r_{I+1}{ }^{N_{I+1}} X_{I+1}-r_{I-1}{ }^{N}{ }_{I-1} X_{I-1}\right)\left(T_{I+1}^{N+1}-T_{I+1}^{N}-T_{I-1}^{N+1}+T_{I-1}^{N}\right) \text {. }
\end{aligned}
$$

Let $\alpha=\frac{x}{2(\Delta r)^{2}}, \delta_{T}=T_{I+1}^{N}-2 T_{I}^{N}+T_{I-1}^{N}, \gamma_{1}=\frac{r_{I+1} N_{I+1}{ }^{N} I+1}{8 r_{I}{ }^{N}(\Delta r)^{2}}$,

$$
\gamma_{-1}=\frac{r_{I-1} N_{I-1} x_{I-1}}{8 r_{I} N_{I}(\Delta r)^{2}} \text {, and let } I+1, I, I-1 \text { be denoted by } 1,0,-1 \text {, }
$$

then

$$
\begin{aligned}
& \left.\frac{1}{\mathrm{rN}_{\mathrm{N}}} \frac{\partial}{\partial r}\left(\mathrm{rN}_{\mathrm{e}} \mathrm{X}_{\mathrm{e}} \frac{\partial \mathrm{T} e}{\partial r}\right)\right|^{\mathrm{N}+\frac{1}{2}}=2 \alpha \delta_{\mathrm{T}}+2 \gamma_{1}\left(\mathrm{~T}_{1}-\mathrm{T}_{-1}\right)^{\mathrm{N}}-2 \gamma_{-1}\left(\mathrm{~T}_{1}-\mathrm{T}_{-1}\right)^{\mathrm{N}}
\end{aligned}
$$

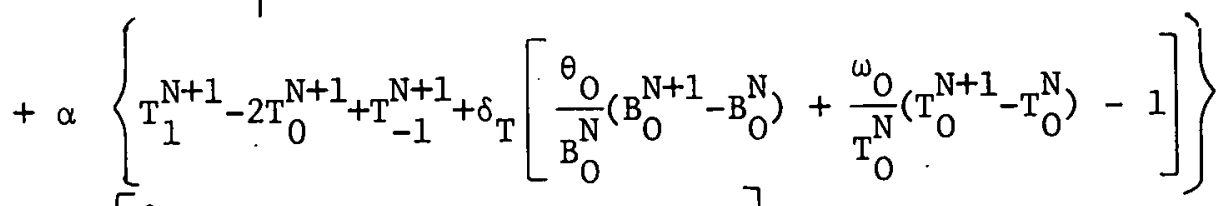

$$
\begin{aligned}
& +\gamma_{1}\left[\frac{\theta_{1}}{B_{1}^{N}}\left(B_{1}^{N+1}-B_{1}^{N}\right)+\frac{\omega_{1}}{T_{1}^{N}}\left(T_{1}^{N+1}-T_{1}^{N}\right)\right]\left(T_{1}-T_{-1}\right) \\
& -\gamma_{-1}\left[\frac{\theta-1}{B_{-1}^{N}}\left(B_{-1}^{N+1}-B_{-1}^{N}\right)+\frac{\omega_{-1}}{T_{-1}^{N}}\left(T_{-1}^{N+1}-T_{1}^{N}\right)\right]\left(T_{1}-T_{-1}\right) \text {. } \\
& +\left(\gamma_{1}-\gamma_{-1}\right)\left[\mathrm{T}_{1}^{\mathrm{N}+1}-\mathrm{T}_{-1}^{\mathrm{N}+1}+\mathrm{T}_{-1}^{\mathrm{N}}-\mathrm{T}_{1}^{\mathrm{N}}\right] \text {. }
\end{aligned}
$$


The plasma variables at the new time $(\mathrm{N}+1)$ and their coefficients can be tabulated:

\section{Component} Coefficient (quantities evaluated at time $N$ )

$\mathrm{T}_{1}^{\mathrm{N}+1}$ $\alpha+\gamma_{1}\left(T_{1}-T_{-1}\right) \frac{\omega_{1}}{T_{1}}+\gamma_{1}-\gamma_{-1}$

$\mathrm{T}_{0}^{\mathrm{N}+1}$ $-2 \alpha+\alpha \delta_{T} \frac{\omega_{0}}{T_{0}}$

$\mathrm{T}_{-1}^{\mathrm{N}+1}$ $\alpha-\gamma_{-1}\left(T_{1}-T_{-1}\right) \frac{\omega}{T_{-1}}-\gamma_{1}+\gamma_{-1}$

$R_{\perp}^{R+1}$ $r_{1} \frac{\dot{\theta}_{1}}{\mathrm{~B}_{1}}\left(I_{1}-\mathrm{r}_{-1}\right)$

$\mathrm{B}_{0}^{\mathrm{N}+1}$ $\alpha \delta_{\mathrm{T}} \frac{{ }_{\mathrm{\theta}}}{\mathrm{B}_{\mathrm{O}}}$ $\mathrm{B}_{-1}^{\mathrm{N}+1}$ $\gamma_{-1} \frac{\theta_{-1}}{\mathrm{~B}_{-1}}\left(\mathrm{~T}_{1}-\mathrm{T}_{-1}\right)$

1 $\alpha \gamma_{T}\left(1-\omega_{0}-\theta_{0}\right)+\left(T_{1}-T_{-1}\right)\left[\gamma_{1}\left(1-\omega_{1}-\theta_{1}\right)-\gamma_{-1}\left(1-\omega_{-1}-\theta_{-1}\right)\right]$

The remainder of the terms in the $T_{p}$ equation can be evaluated in a straightforward fashion.

For the $E$ equation,

$$
\begin{aligned}
& n=n\left(T_{e}, \ldots\right) \sim \mathrm{CT}_{e}^{-3 / 2} \\
& \mathrm{E}^{\mathrm{N}+{ }^{l / 2}} \approx\left(\mathrm{E}+\frac{\Lambda \mathrm{t}}{2} \frac{\partial F_{\mathrm{H}}}{\partial L}\right)=\left(\eta+\frac{\Lambda t}{2} \frac{\partial \eta}{\partial L}\right)\left(j+\frac{\Delta t}{2} \frac{\partial j}{\partial L}-._{D}\right)
\end{aligned}
$$

and

$$
\frac{\partial \eta}{\partial t}=\frac{\partial \eta}{\partial T_{e}} \frac{\partial T_{e}}{\partial L}=-\frac{3}{2} \frac{\eta}{T_{e}} \frac{\partial T_{e}}{\partial t}
$$

Let

$\frac{\partial E}{\partial t}=\frac{1}{2}\left(E^{N}-E\right)$ where in this case $E^{N}$ is the value at the new time. Then to $\operatorname{order}(\Delta t)^{2}$ 


\section{5}

$$
E^{N}+E=2 \eta\left(j-j_{B}\right)+\left[\left(j-j_{B}\right) \frac{\partial \eta}{\partial T_{e}}\left(T_{e}^{N}-T_{e}\right)+n\left(j^{N}-j\right)\right]
$$

and

$$
E^{N}-n j^{N}+\frac{3}{2} \frac{\eta}{T_{e}}\left(j-j_{B}\right) T_{e}^{N}=\frac{3}{2} n\left(j-j_{B}\right)-n j_{B} .
$$


APPENDIX IV

1.

IV. $I$ 
A USER'S MANUAL FOR THE OAK RIDGE

$\therefore \quad$ TOKAMAK TRANSPORT CODE

J. K. Munro H. C. Howe

J. T. Hogan D. E. Arnurius

Appendix IV Microfiche

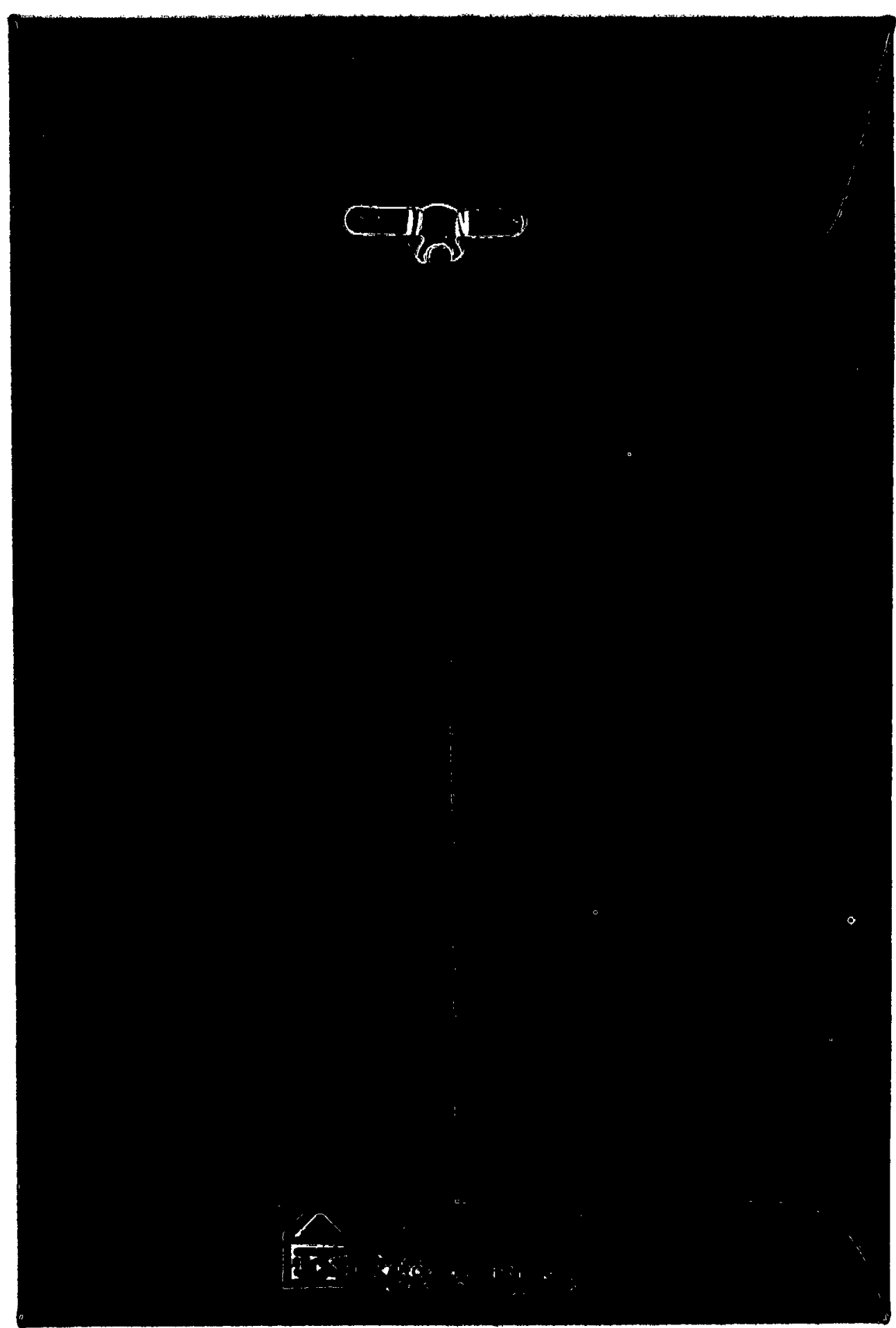


1. J. D. Callen

2. J. F. Clarke

3. R. A. Dory

4-27. J. T. Hogan

28. G. G. Kelley

29. D. G. McAlees

30. 0. B. Morgan

31. M. W. Rosenthal

32-34. Laboratory Records

35. Laboratory Records - ORNL-RC

36. Y-12 Document Reference Section

37-38. Central Research Library

39. Fusion Energy Division Library

40. Fusion Energy Division Reports office

41. ORNL Patent Office

EXTERNAL DISTRIBUTION $\because$

42. Plasma Physics Library, Plasma Physics Laboratory, Princeton Univ., Forrestal Campus, P.0. Box 451, Princeton, NJ 08540

43. Controlled Thermonuclear Research Library, Lawrence Livermore Laboratory, P.0. Box 808, Livermore, CA 94550

44. Q Division Library, c/o F. L. Ribe, Los Alamos Scientific Laboratory, P.0. Box 1.663, Los A1.amos, NM 87544

45. Controlled Thermonuclear Research Library, c/o Weston M. Stacey, Jr., Argonne National Laboratory, 9700 S. Cass Ave., Argonne, IL 60439

46. Magnetic Fusion Energy Computer Center, c/o Dr. John Killeen, Lawrence Livermore Laboratory, P.0. Box 808, Livermore, CA 94550

47. Librarian, Culham Laboratory, U.K. Atomic Energy Authority, Abingdon, Oxon, OX14, 3DB, United Kingdom

48. Ruth Lengye, Blbliothek, Max Planck Institut für Plasmaphysik, 8046 Garching bei München, Federal Republic of Germany

49. Library, Centre de Recherches en Physique des Plasmas, 21 Avenue des Bains, 1007, Lausanne, Switzerland

50. A. M. Dupas, Documentation S.I.G.N., Départment de la Physique du Plasma et de la Fusion Controlée, Association EURATOM-CEA sur la Fusion, Centre d'Etudes Nucleaires, BP 85 Centre Du TRI 38041 Grenoble Cedex (France)

51. Bibliothèque, Service du Confinement des Plasmas, C.E.A., B.P. No. 6, 92, Fontenay-aux-Roses (Seine) France 
52. Library, International Centre for Theoretical Physics, Trieste, Italy

53. Library, Laboratorio Gas Ionizzati, Frascati, Italy

54. V. E. Ivanov, Physical-Technical Institute of the Ukranian Academy of Sciences, Sukhumi, U.S.S.R.

55. L. M. Kovrizhnikh, Lebedev Institute of Physics, Academy of Sciences of the U.S.S.R., Leninsky Prospect 53, Moscow, U.S.S.R.

56. Thermonuclear Laboratory, Kurchatov Institute of Atomic Energy, 46 Ulitsa Kurchatova, P.0. Box 3402, Moscow, U.S.S.R.

57. Library, Institute for Plasma Physics, Nagoya University, Nagoya, Japan 464

58. Library, FOM-Institut voor Plasma-Fysica, Rijnhuizen, Jutphaas, Netherlands

59. Plasma Physics Group, Department of Engineering Physics, Australian National University, P.0. Box 4, Canberra A.C.T. 2600 Australia

60. Thermonuclear Library, Japan Atomic Energy Research Institute, Tokai, Naka, Ibaraki, Japan

61. CTR Reading Room, c/o Prof. Dieter J. Sigmar, Room 37-391, MIT, Cambridge, MA 02139

62. CTR Reading Room, c/o Prof. D. W. Kerst, Dept. of Physics, Sterling Hall, Univ. of Wisconsin, Madison, WI 53706

63. C"l'k Reading Room, c/o Prof. I. B. Bernstein, Yale Univ., New Haven, CT 06510

64. Center for Plasma Physics and Thermonuclear Research, c/o D. W. Ross, Univ. of Texas, Physics Dept., Austin, TX 78712

65. CTR Reading Room, c/o Prof. B. D. Fried, Physics Dept., Univ. of California, Los Angeles, CA 90024

66. CTR Reading Room, c/o Prof. David C. Montgomery, Physics \& Astronomy Dept., Univ. of Iowa, Iowa City, IA 52240

67. Magneto-Fluid-Dynamics Library, Courant Inst. of Math. Sci., New York Univ., 251 Mercer St., New York, NY 10012

68. CTR Reading Room, c/o Prof. Allan N. Kaufman, Physics Dept., Univ. of California, Berkeley, CA 94720

69. CTR Reading Room, c/o Prof. W. B. Thompson, Physics Dept., Univ. of California, San Diego, La Jolla, CA 92037 
70. CTR Reading Room, c/o Prof. Alvin W. Trivelpiece, Dept. of Physics \& Astronomy, Univ. of Maryland, College Park, MD 20742

71. CTR Reading Room, c/o Prof. T. Kammash, 103 Research Admin. Bldg., N. Campus, Univ. of Michigan, Ann Arbor, MI 48105

72. CTR Reading Room, c/o Dr. Ravi N. Sudan, Phillips Hall, Cornell Univ.., Ithaca, NY 14850

73. Prof. Marsha11 N. Rosenbluth, Institute for Advanced Study, Princeton, NJ 08540

74. CTR Reading. Room, c/o Prof. R. Gross, Plasma Research Lab., Columbia Univ., New York, NY 10027

75. CTR Reading Room, c/o Prof. Roy Gould, California Inst. of Tech., M.S. 116-81, Pasadena, CA 91125

76. Dr. Nicholas A. Krall, Science Applications, Inc., P.0. Box 2354, 1200 Prospect St., La Jolla, CA 92037

77. CTR Reading Room, c/o Dr. Jay P. Boris, Plasma Physics, Naval Research Laboratory, Washington, DC 20390

78. Professor A. Simon, Dept. of Mechanica1 \& Aerospace Sciences, University of Rochester, Rochester, NY 14627

79. CTR Library, c/o Dr. Alan F. Haught, United lechnologies Research Labs, East Hartford, CI 06108

80. Dr. Robert E. Price, Division of Magnetic Fusion Energy, G-234, Energy Research and Development Administration, Washington, DC 20545

81. Dr. Bennett Miller, Division of Magnetic Fusion Energy, G-234, Energy Research and Development Administration, Washington, DC 20545

82. Dr. Arthur Sleeper, Division of Magnetic Fusion Energy, G-234, Energy Research and Development Administration, Washington, DC 20545

83. Dr. Walter Sadowski, Division of Magnetic Fusion Energy, G-234, Energy Research and Development Administration, Washington, DC 20545

84. Dr. D. H. Priester, Division of Magnetic Fusion Energy, G-234, Energy Research and Development Administration, Washington, DC 20545

85. Mr. E. E. Kintner, Division of Magnetic Fusion Energy, G-234, Energy Research and Development Administration, Washington, DC 20545 
86. Dr. L. D. Pearlstein, L-388, Lawrence Livermore Laboratory, P.0. Box 808, Livermore, CA 94550

87. Dr. J. P. Friedberg, Los Alamos Scientific Laboratory, Los Alamus NM 87544

88. Dr. David J. Rose, Dept. of Nuclear Engineering, MIT, Cambridge, MA 02139

89. Dr. Gareth E. Guest, Genera1 Atomic Co., P.o. Box 81608, San Diego, CA 92138

90. Dr. Claude Mercier, Service du Theorie des Plasmas, Centre d'Etudes Nucleaires, Fontenay-aux-Roses (Seine) France

91. Dr. J. B. Taylor, Culham Laboratory, UKAEA Ab1ngdon, Oxon, OX14 3DB, United Kingdom

92. Dr. D. Hfirsch, Institute for Plasma Physics, 8046 Garching bei München, Federal Republic of Germany

93. Dr. V. D. Shafranov, I. V. Kurchatov Inst. of Atomic Energy, 46 Ulicsa Kurchatova, P.0. Box 3402, Moscow, U.S.S.R.

94. Dr. Harold Grad, Courant Inst. of Math. Sci., New York Univ., 251 Mercer St., New York, NY 10012

95. Dr. J. G. Cordey, Culham Laboratory, UKAEA, Abingdon, Oxon, 0X14, 3DB, United Kingdom

96. Dr. David Baldwin, L-388, Lawrence Livermore Lab., P.0. Box 808, Livermore, CA 94550

y). Prof. Bruno Coppi, Dept. of Physics, MIT, Cambridge, MA 02139

98. Dr. Harold P. Furth, Princeton Plasma Physics Lab., Princeton Univ., P.O. Box 451, Princeton, NJ 08540

99. Dr. Paul H. Rutherford, Princeton Plasma Physirs T.ab., Princeton Univ., Princeton, NJ 08540

100. Dr. Herbert H. Woodson, Dept. of Electrical Engineering, Univ. of Texas, Austin. TX $7871 ?$

1U1. Research \& Technical Support Div., Oak Ridge Operations, Energy Research and Development Administration, P. O. Box E, Oak Ridge, TN 37830

102-128. Technical Information Center, Fnergy Research and Devclopment Administration, P.0. Box 62, Oak Ridge, TN 37830 